\title{
Modeling that predicts elementary particles and explains data about dark matter, early galaxies, and the cosmos
}

Thomas J. Buckholtz

Ronin Institute, Montclair, New Jersey 07043, USA

\begin{abstract}
We try to solve three decades-old physics challenges. List all elementary particles. Describe dark matter. Describe mechanisms that govern the rate of expansion of the universe. We propose new modeling. The modeling uses extensions to harmonic oscillator mathematics. The modeling points to all known elementary particles. The modeling suggests new particles. Based on those results, we do the following. We explain observed ratios of dark matter amounts to ordinary matter amounts. We suggest details about galaxy formation. We suggest details about inflation. We suggest aspects regarding changes in the rate of expansion of the universe. We interrelate the masses of some elementary particles. We interrelate the strengths of electromagnetism and gravity. Our work seems to offer new insight regarding applications of harmonic oscillator mathematics. Our work seems to offer new insight regarding three branches of physics. The branches are elementary particles, astrophysics, and cosmology.
\end{abstract}

Keywords: Beyond the Standard Model, Dark matter, Dark energy, Inflation, Galaxy evolution, Rate of expansion of the universe, Quantum gravity, Harmonic oscillator, Mathematical physics

October 20, 2020

Copyright (c) 2020 Thomas J. Buckholtz

\section{Contents}

\begin{tabular}{|lll}
\hline & Introduction and summary & 4
\end{tabular}

\begin{tabular}{|ll|l}
2 & Methods & $\mathbf{7}$
\end{tabular}

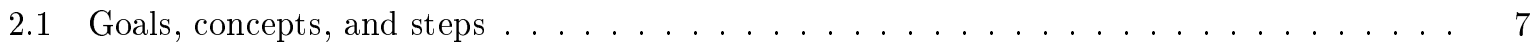

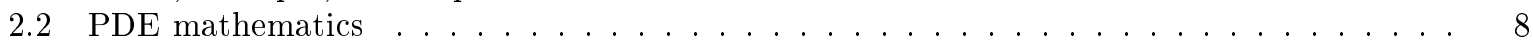

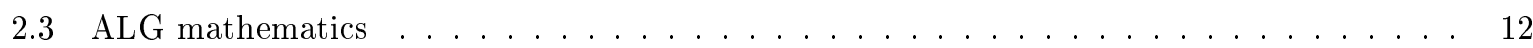

2.4 Relationships between applications of various types of models $\ldots \ldots \ldots \ldots \ldots \ldots$

$2.5 \quad$ A method that organizes properties of elementary particles and other objects $\ldots \ldots \ldots$

\begin{tabular}{|lll}
\hline & Results: elementary particles & 16
\end{tabular}

3.1 Summary: a table of known and suggested elementary particles . . . . . . . . . . . . . 20

3.2 Modeling leading to the table of elementary particles $\ldots \ldots \ldots \ldots \ldots \ldots$. . . . . . . 22

3.2 .1 Proposed elementary particle modeling (or, PEPM) . . . . . . . . . . . . . . . 24

3.2 .2 Patterns regarding properties of known elementary particles . . . . . . . . . . . . . 29

3.2 .3 Some applications of PDE mathematics $\ldots \ldots \ldots \ldots \ldots \ldots$

3.2 .4 PDE aspects of proposed elementary particle modeling . . . . . . . . . . . . . 29

$3.2 .5 \quad$ PDE modeling regarding simple particles $\ldots \ldots \ldots \ldots$. . . . . . . . . . . 31

3.2 .6 Concepts regarding representations for photons $\ldots \ldots \ldots \ldots \ldots \ldots$

3.2 .7 ALG representations for elementary particles $\ldots \ldots \ldots \ldots \ldots \ldots \ldots$

3.2 .8 Some PEPM concepts re models that output the list of elementary particles. . . . 43

Email address: Thomas.Buckholtz@RoninInstitute.org (Thomas J. Buckholtz) 
4 Results: properties of elementary particles and multicomponent objects 43

4.1 Summary: a table of properties of elementary particles and multicomponent objects . . . 43

$4.2 \quad$ Modeling regarding properties of elementary particles and multicomponent objects . . . . 44

$4.2 .1 \quad$ Kinematics conservation laws . . . . . . . . . . . . . . . 44

4.2 .2 G-family phenomena, including electromagnetism and gravity . . . . . . . . . . . . 47

4.2.3 Conservation of lepton number minus baryon number . . . . . . . . . . . . . . . 52

4.2 .4 Refraction and similar phenomena . . . . . . . . . . . . . . . . 53

4.2 .5 Gluon interactions . . . . . . . . . . . . . . . . . . . . . . . . 54

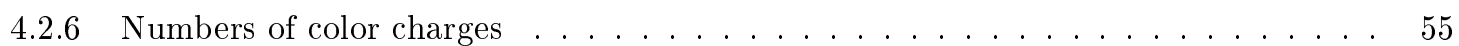

4.2 .7 Interactions involving jay bosons . . . . . . . . . . . . . . . . . . . . . 55

4.2.8 A series of formulas for lengths, including the Planck length . . . . . . . . . . . . 55

4.2 .9 Ranges for interactions that correlate with elementary bosons . . . . . . . . . . . 55

4.2.10 Correlations between simple particles and G-family and U-family ALG solutions . 57

$4.3 \quad$ Predictions and correlations regarding properties of elementary particles . . . . . . . . . 58

4.3.1 The masses of the $\mathrm{W}, \mathrm{Z}$, and Higgs bosons $\ldots \ldots \ldots \ldots$. . . . . . . . . . . . . . . . . . . . . . . .

4.3.2 The masses of the aye boson and jay bosons . . . . . . . . . . . . . . . . . . . 60

4.3.3 Possible masses of the tweak bosons . . . . . . . . . . . . . . . . . . . . . 60

4.3 .4 A prediction for the tauon mass . . . . . . . . . . . . . . . . . . 61

4.3.5 The masses of quarks and charged leptons . . . . . . . . . . . . . . . . . . . . 61

4.3.6 The relative strengths of electromagnetism and gravity. . . . . . . . . . . . . . . . 64

4.3.7 Channels and interactions that involve G-family bosons . . . . . . . . . . . . . . 66

4.3.8 The relative strengths of $2 \mathrm{G} 2,2 \mathrm{G} 24, \mathrm{~W}$-boson, and other spin-one interactions . . 66

4.3.9 Relative strengths of interactions re G-family bosons with spins of at least two . . 67

4.3 .10 Roles of the fine-structure constant . . . . . . . . . . . . . . 68

4.3 .11 Numbers of fermion generations . . . . . . . . . . . . . . . . . . . . 68

4.3.12 Ongoing modeling estimates for the sum of neutrino masses . . . . . . . . . . . . 68

4.3.13 Models that might estimate an ongoing modeling sum of neutrino masses . . . . . 69

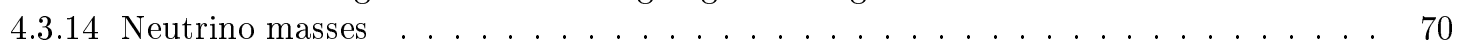

4.3 .15 A possible lack of electric dipole moments for elementary particles . . . . . . . . . 71

4.3.16 A possible lack of neutrino asymmetry . . . . . . . . . . . . . . . 71

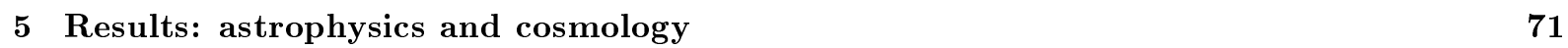

$5.1 \quad$ Summary: a table of predictions and explanations re astrophysics and cosmology . . . . . 71

$5.2 \quad$ Modeling pertaining to astrophysics and cosmology . . . . . . . . . . . . . . . 72

5.2.1 Modeling that describes dark matter particles . . . . . . . . . . . . . . . . . 72

5.2.2 Spans for simple particles, components of root forces, and some objects . . . . . . 74

5.2.3 Dark matter to ordinary matter ratios that modeling might predict or explain. . . 78

5.2 .4 Some properties of isomers of quarks and charged leptons . . . . . . . . . . 78

5.2.5 Possible differences regarding the evolution of dark matter isomers . . . . . . . . . 82

$5.3 \quad$ Predictions and explanations regarding astrophysics and cosmology . . . . . . . . . . . . . 83

5.3.1 A specification for dark matter and ordinary matter . . . . . . . . . . . . . . . 83

5.3 .2 Densities of the universe . . . . . . . . . . . . . . . . . 84

$5.3 .3 \quad$ DM to OM density of the universe ratios inferred from data regarding CMB. . . . 84

5.3.4 Constancy of actual density of the universe ratios re DM and OM . . . . . . . 85

5.3.5 A possibly DM effects to OM effects ratio inferred from data regarding CMB . . . 85

5.3 .6 The rate of expansion of the universe . . . . . . . . . . . . . 85

5.3.7 Phenomena - including the creation of isomers - before through just after inflation 86

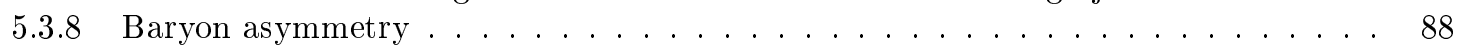

$5.3 .9 \quad$ Filaments and baryon acoustic oscillations . . . . . . . . . . . . . . . 89

5.3.10 Amounts of clumping for large clumps of ordinary matter gas and of dark matter . 89

5.3.11 Galaxy clusters - ratios of dark matter to ordinary matter . . . . . . . . . . . 9 90

5.3.12 Galaxy clusters - an explanation for aspects of the Bullet Cluster . . . . . . . . . . 90

5.3 .13 Galaxies - formation . . . . . . . . . . . . . . . . . 91

5.3 .14 Galaxies - ratios of dark matter stuff to ordinary matter stuff . . . . . . . . . . . . 92

5.3 .15 Aspects regarding some components of galaxies . . . . . . . . . . . . . . . . . . . . 96

5.3 .16 Dark matter effects within the Milky Way galaxy . . . . . . . . . . . . . . . . . . 96

5.3 .17 High-mass neutron stars . . . . . . . . . . . . . . . . . . . 96

5.3 .18 Dark energy density . . . . . . . . . . . . . . . . . . . . . 97 
6 Discussion: core ongoing modeling and core proposed modeling $\quad 97$

6.1 The elementary particle Standard Model . . . . . . . . . . . . . . . . . . . . . . 98

6.2 Concordance cosmology . . . . . . . . . . . . . . . . . . . . . . . . . 98

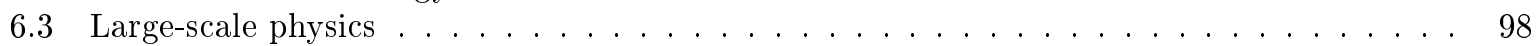

6.4 Quantum electrodynamics regarding positronium . . . . . . . . . . . . . . . . . . . 99

6.5 The masses of $1 \mathrm{R} \otimes 2 \mathrm{U}$ hadron-like dark matter particles . . . . . . . . . . . . . . . . . . . 99

6.6 Relationships between core proposed modeling and core ongoing modeling . . . . . . . . . 100

\begin{tabular}{|lll}
\hline 7 & Discussion: unverified ongoing modeling and core proposed modeling & 101
\end{tabular}

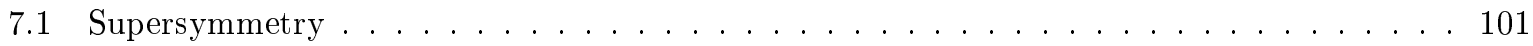

7.2 String theory . . . . . . . . . . . . . . . . . . . . . . . . 101

7.3 Theory of everything . . . . . . . . . . . . . . . . . . . . . . . . 101

7.4 The strong CP problem . . . . . . . . . . . . . . . . . . . . . . . . . . . . 102

7.5 Possible lacks of some unverified ongoing modeling elementary particles . . . . . . . . . . 102

7.5 .1 A possible lack of magnetic monopoles . . . . . . . . . . . . . . . . . 102

7.5.2 A possible lack of axion elementary particles . . . . . . . . . . . . . . . . 105

8 Discussion: supplementary proposed modeling and core ongoing modeling 105

$8.1 \quad$ Modeling that proposed modeling suggests regarding dynamics . . . . . . . . . . . . 105

8.1.1 CQFM interaction vertices that involve simple particles and root forces . . . . . . 105

8.1.2 Supplementary proposed modeling dynamics models re multicomponent objects. . 106

$8.1 .3 \quad$ Dynamics models for hadron-like particles . . . . . . . . . . . . . . . 108

8.1 .4 Dynamics models for nuclear physics . . . . . . . . . . . . . . . . . . . . . 108

8.1 .5 Dynamics models for atomic physics . . . . . . . . . . . . . . . . . . . . . 109

8.1 .6 Dynamics models for quantum transitions . . . . . . . . . . . . . . . . . . . . . 109

8.2 Possible applications of proposed modeling KMS modeling . . . . . . . . . . . . . . . . . . 109

$8.2 .1 \quad$ A possible lack of a neutron electric dipole moment . . . . . . . . . . . . . . . . . . 109

8.2 .2 Anomalous magnetic dipole moments . . . . . . . . . . . . . . . . . . 110

8.3 Possibilities to complement ongoing modeling classical physics . . . . . . . . . . . . . . . . 112

8.3.1 Possibilities for using Newtonian modeling in place of general relativity . . . . . . 112

8.3.2 $\quad$ Possibilities for extending aspects related to Maxwell's equations . . . . . . . . . . 112

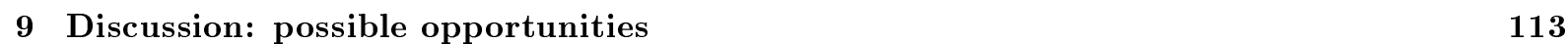

9.1 Possible opportunities for experimental or observational research . . . . . . . . . . . . . . 113

9.1.1 Possible themes for experiments or observations . . . . . . . . . . . . . . . . . 113

9.1.2 $\quad$ Possibilities for detecting or inferring aye and jay bosons. . . . . . . . . . . . . . . 114

9.1.3 Possibilities for directly detecting dark matter . . . . . . . . . . . . . . . . . . . 115

9.2 Possible opportunities regarding harmonic oscillator mathematics . . . . . . . . . . . . . . 116

9.2.1 Possible opportunities re PDE harmonic oscillator mathematics . . . . . . . . . . . 116

9.2.2 Possible opportunities re ALG harmonic oscillator mathematics and modeling. . . 117

9.3 Possible opportunities to develop deeper insight $\ldots \ldots \ldots \ldots$. . . . . . . . . . . . 117

9.3 .1 Numbers of dimensions $\ldots \ldots \ldots \ldots \ldots \ldots$

9.3 .2 Arrow of time . . . . . . . . . . . . . . . . . . . . 117

$9.3 .3 \quad$ Inertial mass and gravitational mass . . . . . . . . . . . . . . . . . . . . 118

9.3.4 Notions that might link physics constants and modeling . . . . . . . . . . . . . . . 118

9.3 .5 Entropy $\ldots \ldots \ldots \ldots \ldots \ldots \ldots$

\begin{tabular}{|rr}
\hline 10 Concluding remarks & 120
\end{tabular}

\begin{tabular}{|rr}
\hline Acknowledgments & 120
\end{tabular}

\begin{tabular}{lr}
\hline References & 121
\end{tabular} 


\section{Introduction and summary}

We offer modeling that may solve at least the following three physics challenges. List all elementary particles. Describe dark matter. Explain some seemingly unresolved aspects regarding the rate of expansion of the universe.

The modeling outputs the elementary particle Standard Model particle set and suggests additional elementary particles. The modeling suggests a well-specified description of dark matter particles. The modeling adds aspects to concordance cosmology.

This essay discusses relationships between data, so-called ongoing modeling, and so-called proposed modeling. The data features the domains of elementary particle physics, astrophysics, and cosmology. Ongoing modeling denotes established physics modeling and unverified modeling that other people propose. Proposed modeling denotes our work.

Each of ongoing modeling and proposed modeling includes a core component and another component. Core ongoing modeling includes established modeling regarding motion, the Standard Model particle set, and concordance cosmology. Ongoing modeling also includes unverified models such as supersymmetry. Core proposed modeling outputs a set of elementary particles that includes and adds to the Standard Model elementary particle set. Supplementary proposed modeling includes modeling - regarding motion - that has some similarities to quantum field theory.

Table 1 lists some goals that we have for proposed modeling.

The core of this essay has bases in synergies between core ongoing modeling and core proposed modeling. For example, each one of core ongoing modeling and core proposed modeling embraces symmetries that correlate with, for example, conservation of momentum and conservation of angular momentum.

Our work progressed through three phases. Each later phase enriched methods and results from prior phases. Phase one pursued the following two goals. Explain three eras in the rate of expansion of the universe. Explain the ratio of dark matter density of the universe to ordinary matter density of the universe. Phase two pursued the following two goals. Develop and use a model that outputs the list of all known elementary particles and a set of well-specified suggested elementary particles. Describe dark matter. Phase three pursued the following goal. Explain ratios, that pertain to galaxy clusters and to galaxies, of dark matter amounts to ordinary matter amounts.

Table 2 summarizes some of the results that our modeling produces. (Table $27 \mathrm{~b}$ notes the new property - isomer or isomers - that pertains regarding elementary particles. Discussion regarding table 27 points to additional information about isomers.)

Table 3 points to tables that summarize some results from proposed modeling.

Table 4 discusses relationships between some aspects of ongoing modeling and some aspects of proposed modeling.

The following remarks provide perspective about this essay.

Reference [1] suggests standards regarding discussing modeling. Regarding individual models, we discuss correlations with data, limits of applicability, opportunities to make improvements, unresolved aspects, and alternatives. Regarding collections of models, we discuss possible synergies between models and possible discord between models.

This essay makes correlations between aspects of data, ongoing modeling, and proposed modeling. Such correlations can consider that aspects of one model do not necessarily equal similar aspects of another model. Wording of the form AA correlates with BB does not necessarily imply concepts such as AA equals BB or AA implies BB.

Table 1: Some goals for proposed modeling

Goal

- Explain data that ongoing modeling seems not to explain.

- Predict data that people can (at least eventually) verify or refute.

- Develop and use models that are compatible with essentially all relevant currently known data.

- Develop and use models that are compatible with core ongoing modeling. (The models might point to limits of applicability for some aspects of core ongoing modeling.)

- Develop and use models that people might find, based on notions such as preponderance of evidence, to be more compelling than aspects of current unverified ongoing modeling.

- Develop and use models - that people might find to be compelling - regarding relevant topics that ongoing modeling seems not to address. 
Table 2: Some results

\begin{tabular}{|c|c|c|}
\hline Field & Area & Results \\
\hline Mathematics & Harmonic oscillators & Solutions that lie below traditional ground states \\
\hline Applied mathematics & Mathematical physics & $\begin{array}{l}\text { Models that catalog and interrelate properties of } \\
\text { elementary particles and other objects }\end{array}$ \\
\hline Applied mathematics & Mathematical physics & $\begin{array}{l}\text { Models that output known and new elementary } \\
\text { particles }\end{array}$ \\
\hline Elementary particles & Existence & A list of possibly all elementary particles \\
\hline Elementary particles & Properties & A new property \\
\hline Astrophysics & Dark matter & A description of dark matter \\
\hline Astrophysics & Dark matter & $\begin{array}{l}\text { Explanations for ratios of dark matter amounts } \\
\text { to ordinary matter amounts }\end{array}$ \\
\hline Cosmology & $\begin{array}{l}\text { Dark energy negative } \\
\text { pressure }\end{array}$ & $\begin{array}{l}\text { An explanation for three eras in the rate of } \\
\text { expansion of the universe }\end{array}$ \\
\hline Cosmology & Early universe & Details regarding the inflationary epoch \\
\hline Cosmology & Early universe & A possible cause for baryon asymmetry \\
\hline Astrophysics & Galaxy evolution & $\begin{array}{l}\text { Predictions and explanations regarding galaxy } \\
\text { formation }\end{array}$ \\
\hline Physics & $\begin{array}{l}\text { Properties of } \\
\text { elementary particles }\end{array}$ & $\begin{array}{l}\text { Relationships between masses of elementary } \\
\text { particles }\end{array}$ \\
\hline Physics & $\begin{array}{l}\text { Properties of } \\
\text { elementary particles }\end{array}$ & $\begin{array}{l}\text { A relationship between the strengths of } \\
\text { electromagnetism and gravity }\end{array}$ \\
\hline
\end{tabular}

Table 3: Tables that summarize some results from proposed modeling

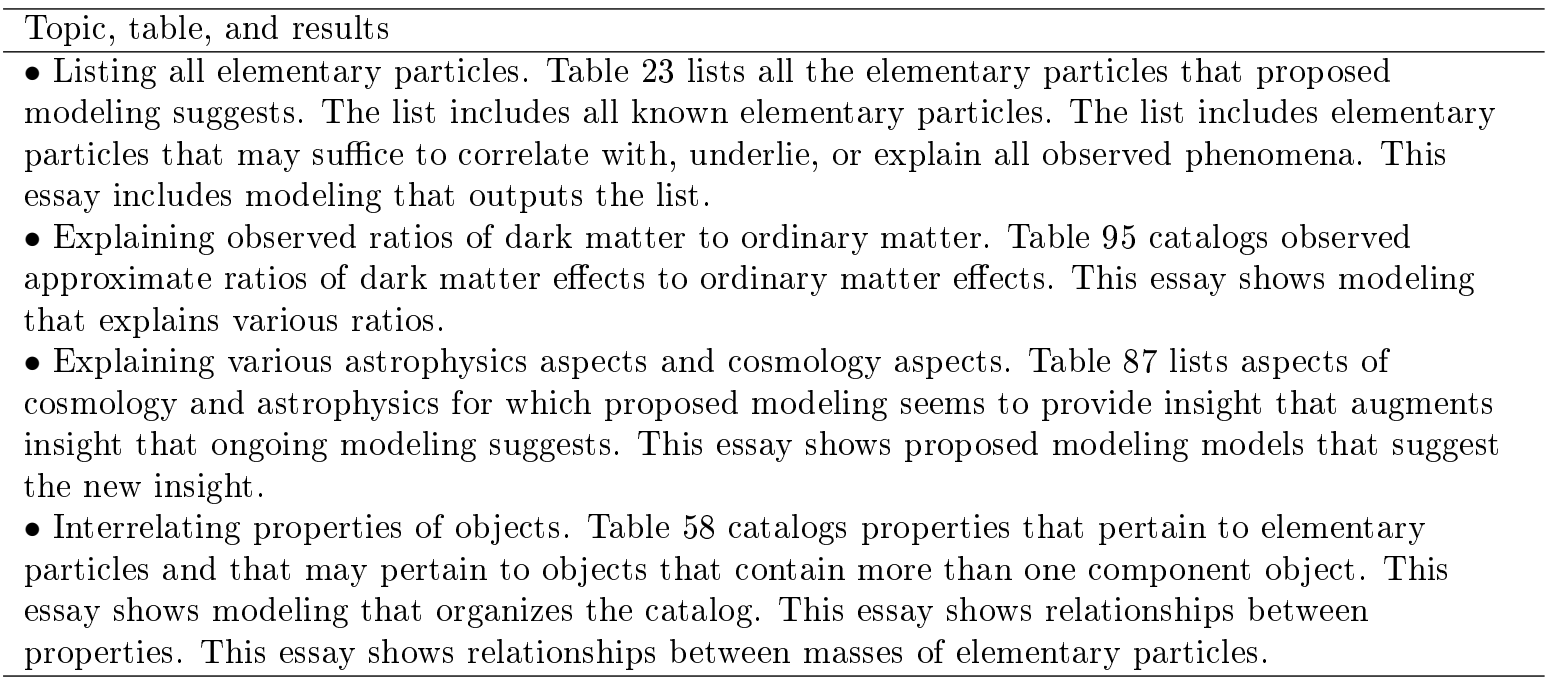


Table 4: Relationships between some aspects of ongoing modeling and some aspects of proposed modeling

(a) Core ongoing modeling and core proposed modeling

Aspect of core ongoing modeling - Discussion based on core proposed modeling

- The elementary particle Standard Model - Proposed modeling outputs a list of elementary particles. The list includes all Standard Model elementary particles that people have found. The list suggests other elementary particles. Our work suggests possibilities for adding the suggested particles to the Standard Model.

- The Lambda-CDM (cosmology) model - Proposed modeling embraces observed aspects (of nature) that the Lambda-CDM model embraces. Proposed modeling suggests explanations for some observed aspects for which people have yet to agree on explanations. Proposed modeling suggests aspects that people might want to add to the Lambda-CDM model.

- Dark matter - Proposed modeling suggests that much dark matter has some similarities to unverified ongoing modeling notions of so-called WIMPs (or, weakly interacting massive particles). Unlike would-be WIMPs, this dark matter features hadron-like particles (which include elementary particles) that people would not consider to be elementary particles.

- Modeling regarding large-scale phenomena - People allude to possible problems regarding using the Hubble constant, models that compute pressures based on densities, and general relativity to model some of the largest-scale phenomena that people observe. Proposed modeling points to reasons why such modeling may not apply adequately accurately to some aspects of large-scale phenomena.

- Modeling regarding motion - People can use core proposed modeling with modeling regarding motion that comports with conservation of energy, conservation of angular momentum, and conservation of momentum. Each one of core ongoing modeling and supplementary proposed modeling includes such modeling regarding motion.

(b) Unverified ongoing modeling and core proposed modeling

Aspect of unverified ongoing modeling - Discussion based on core proposed modeling

- Quantum gravity - Proposed modeling outputs (rather straightforwardly) a model of quantum gravity. That modeling and other concepts that this essay discusses seem to point to difficulties regarding trying to describe quantum gravity by quantizing aspects of general relativity.

- Supersymmetry - The proposed modeling list of elementary particles may suffice to explain phenomena that led people to suggest supersymmetry. The list does not exhibit supersymmetry. There may be little further physics need for people to explore supersymmetry.

(c) Core ongoing modeling and supplementary proposed modeling

Aspect of core ongoing modeling - Discussion based on supplementary proposed modeling

- Quantum field theory - Supplementary proposed modeling suggests a somewhat parallel to ongoing modeling QFT (or, quantum field theory). The two types of modeling differ from each other. For example, the proposed modeling parallel to QFT features modeling that is quadratic in energy, whereas ongoing modeling QFT features modeling that is linear in energy. Proposed modeling that somewhat parallels QFT might provide or point to useful bases for modeling, for example regarding anomalous magnetic moments or regarding nuclear physics. 
Table 5: Goals for PEPM (or, proposed elementary particle modeling)

PEPM should include modeling that ...

- Points to all known elementary particles and possibly to all unknown elementary particles.

- Outputs representations correlating with the elementary particles.

- Outputs information about properties of the elementary particles.

- Outputs information about interactions in which elementary particles participate.

- Embraces conservation laws pertaining to motion.

- Embraces core ongoing modeling regarding motion.

- Helps explain data that ongoing modeling seems not to explain.

Table 6: Concepts and steps underlying development of PEPM (with the word particles denoting the two-word term elementary particles)

Concepts and steps

- Correlations exist between spins and numbers of similar particles.

- So-called PDE modeling, based on math that echoes those correlations, can be useful.

- PDE modeling uses partial differential equations pertaining to harmonic oscillators.

- PDE modeling mathematically correlates allowed spins with three spatial dimensions.

- PDE modeling uses information about some particles to output aspects of other particles.

- So-called ALG modeling features ladder operators pertaining to harmonic oscillators.

- ALG modeling uses symmetries pertaining to harmonic oscillators.

- ALG modeling has bases in models that correlate with the excitement of boson states.

- ALG modeling outputs representations that correlate with particles.

- ALG modeling points to symmetries that correlate with properties of particles.

- ALG modeling augments, regarding particle properties, PDE modeling.

- ALG modeling proposes new property-centric conservation laws.

- ALG modeling points to symmetries that correlate with properties of interactions.

- ALG modeling embraces symmetries correlating with kinematics conservation laws.

- ALG modeling helps bridge between proposed modeling and ongoing modeling.

- ALG modeling bridging includes aspects correlating with motion.

This essay cites references regarding data. For some aspects of mathematics and modeling, this essay does not cite references. Perhaps, reference [2] points to precedent for not necessarily citing references regarding mathematics and modeling as well as to precedent for discussing context.

\section{Methods}

We provide perspective about our development and use of proposed modeling.

\subsection{Goals, concepts, and steps}

We use the four-word term proposed elementary particle modeling to describe a core of our work. The acronym PEPM abbreviates the four-word term proposed elementary particle modeling.

Table 5 suggests goals for PEPM. Interactions can change, regarding objects in general, each of internal properties and motion.

Our work contributes to each of the goals that table 5 lists.

Ongoing modeling does not necessarily achieve the first few goals. Development of ongoing modeling has tended to produce modeling regarding motion without necessarily completely knowing the nature of objects that move or without necessarily completely cataloging types of objects that move.

Goals that table 5 lists correlate with potential synergy between proposed modeling and ongoing modeling. Together, proposed modeling and ongoing modeling seem to explain data that ongoing modeling seems not to explain.

Table 6 notes concepts and steps that underlie this essay's development of PEPM. (Regarding the correlation between spin and number of particles, see table 29c) The acronym PDE abbreviates the three-word term partial differential equation. The three-letter term ALG stands for the word algebraic.

We provide perspective about harmonic oscillator mathematics.

Mathematics pertaining to harmonic oscillators includes two types of expressions. PDE modeling features solutions that feature sums of terms of the form that equation (1) shows. The symbol $x$ denotes 
Table 7: Possible values for and assumptions about $\Psi(r), \Omega_{S A}, D$, and $\nu_{S A}$

(a) Possible values, plus some assumptions that ongoing modeling tends to embrace

\begin{tabular}{lll}
\hline Symbol & Possible values & Assumption that ongoing modeling tends to embrace \\
\hline$\Psi(r)$ & Complex numbers & Complex numbers \\
$\Omega_{S A}$ & Complex number & Non-negative integer \\
$D$ & Complex number & Positive integer \\
$\nu_{S A}$ & Complex number & Non-negative integer \\
\hline
\end{tabular}

(b) Other aspects that ongoing modeling tends to embrace

Aspect that ongoing modeling tends to embrace

- The symbol $\Psi(r)$ denotes a function of angular coordinates, as well as of $r$.

- The symbol $\Omega_{S A}$ correlates with aspects correlating with angular coordinates.

- The symbol $D$ correlates with a number of dimensions.

- The symbol $\nu_{S A}$ might correlate with an energy level.

a continuous variable. ALG modeling features solutions that feature one or more terms, with each term being a product of one or more factors of the form that equation (2) shows. The occupation number $n$ is an integer.

$$
\begin{gathered}
x^{\nu} \exp \left(x^{-2}\right) \\
\mid n>
\end{gathered}
$$

\subsection{PDE mathematics}

We explore mathematics underlying PDE modeling.

Equations (3) and (4) correlate with an isotropic quantum harmonic oscillator. Here, $r$ denotes the radial coordinate and has dimensions of length. The parameter $\eta_{S A}$ has dimensions of length. The parameter $\eta_{S A}$ is a non-zero real number. The magnitude $\left|\eta_{S A}\right|$ correlates with a scale length. Each of $\xi_{S A}$ and $\xi_{S A}^{\prime}$ is a constant. The symbol $\Psi(r)$ denotes a function of $r$. The symbol $\nabla_{r}^{2}$ denotes a Laplacian operator. The symbol $\Omega_{S A}$ is a constant. We associate the term SA-side with this use of symbols and mathematics. We anticipate that the symbols used correlate with spatial aspects of some physics modeling. We anticipate that TA-side symbols and mathematics pertain for - and correlate with temporal aspects of - some physics modeling.

$$
\begin{gathered}
\xi_{S A} \Psi(r)=\left(\xi_{S A}^{\prime} / 2\right)\left(-\left(\eta_{S A}\right)^{2} \nabla_{r}^{2}+\left(\eta_{S A}\right)^{-2} r^{2}\right) \Psi(r) \\
\nabla_{r}^{2}=r^{-(D-1)}(\partial / \partial r)\left(r^{D-1}\right)(\partial / \partial r)-\Omega_{S A} r^{-2}
\end{gathered}
$$

We explore solutions that pertain for the range that equation (5) shows.

$$
0<r<\infty
$$

We consider solutions of the form that equation (6) shows.

$$
\Psi(r) \propto\left(r / \eta_{S A}\right)^{\nu_{S A}} \exp \left(-r^{2} /\left(2\left(\eta_{S A}\right)^{2}\right)\right), \text { with }\left(\eta_{S A}\right)^{2}>0
$$

Table 7 discusses possible values for and assumptions about $\Psi(r), \Omega_{S A}, D$, and $\nu_{S A}$. Our work does not necessarily automatically embrace assumptions that ongoing modeling tends to embrace.

Table 8 provides details that lead to solutions that equations $(7)$ and $(8)$ characterize. We consider equations (3), (4), and (6). The table assumes, without loss of generality, that $\left(\xi_{S A}^{\prime} / 2\right)=1$ and that $\eta_{S A}=1$. More generally, we assume that each of the four terms $K$ and each of the two terms $V$ includes appropriate appearances of $\left(\xi_{S A}^{\prime} / 2\right)$ and $\eta_{S A}$. The term $V_{+2}$ correlates with the rightmost term in equation (3). The term $V_{-2}$ correlates with the rightmost term in equation (4). The four $K$ terms correlate with the other term to the right of the equals sign in equation (4). The sum of the two $K_{0}$ terms correlates with the factor $D+2 \nu_{S A}$ in equation (7).

Equations (7) and (8) characterize solutions. The parameter $\eta_{S A}$ does not appear in these equations. 
Table 8: Terms correlating with an SA-side PDE equation (assuming that $\left(\xi_{S A}^{\prime} / 2\right)=1$ and $\eta_{S A}=1$ )

\begin{tabular}{ccccc}
\hline Term $/ \exp \left(-r^{2} / 2\right)$ & $\begin{array}{r}\text { Symbol } \\
\text { for term }\end{array}$ & $\begin{array}{c}\text { Change in } \\
\text { power of } r\end{array}$ & Non-zero unless ... & Notes \\
\hline$-r^{\nu_{S A}+2}$ & $K_{+2}$ & +2 & - & Cancels $V_{+2}$ \\
$\left(D+\nu_{S A}\right) r^{\nu_{S A}}$ & $K_{0 a}$ & 0 & $D+\nu_{S A}=0$ & - \\
$\nu_{S A} r^{\nu_{S A}}$ & $K_{0 b}$ & 0 & $\nu_{S A}=0$ & - \\
$-\nu_{S A}\left(\nu_{S A}+D-2\right) r^{\nu_{S A}-2}$ & $K_{-2}$ & -2 & $\nu_{S A}=0$ or & Cancels $V_{-2}$ \\
& & -2 & $\left(\nu_{S A}+D-2\right)=0$ & \\
$\Omega_{S A} r^{\nu_{S A}-2}$ & $V_{-2}$ & -2 & $\Omega_{S A}=0$ & Cancels $K_{-2}$ \\
$r^{\nu_{S A}+2}$ & $V_{+2}$ & +2 & - & Cancels $K_{+2}$ \\
\hline
\end{tabular}

$$
\begin{aligned}
& \xi_{S A}=\left(D+2 \nu_{S A}\right)\left(\xi_{S A}^{\prime} / 2\right) \\
& \Omega_{S A}=\nu_{S A}\left(\nu_{S A}+D-2\right)
\end{aligned}
$$

We explore the topic of normalization regarding $\Psi(r)$.

In ongoing modeling, people consider that $\Psi(r)$ normalizes if and only if equation (9) pertains. The symbol $(\Psi(r))^{*}$ denotes the complex conjugate of $(\Psi(r))$.

$$
\int_{0}^{\infty}(\Psi(r))^{*} \Psi(r) r^{D-1} d r<\infty
$$

Our work embraces somewhat the same concept - as ongoing modeling embraces - regarding normalization. The difference in the domain for $r$ (that is, $0<r<\infty$ for our work versus $0 \leq r<\infty$ for ongoing modeling) is not material for this essay. For essentially the entire remainder of this essay, we assume that equation (10) pertains. (For a complex number $z$, the expression $z=\Re(z+i \Im(z)$ pertains. The expression $\Re(z)$ denotes the real part of $z$. The expression $\Im(z)$ denotes the imaginary part of $z$. The symbol $i$ denotes the positive square root of the number -1.) We take the liberty to assume that the normalization criterion that equation (9) defines pertains for any real number $D$.

$$
\Im(D)=0
$$

For essentially the entire remainder of this essay, we assume that equation (11) pertains.

$$
\Im\left(\nu_{S A}\right)=0
$$

Equation (12) correlates with the domains of $D$ and $\nu_{S A}$ for which normalization pertains for $\Psi(r)$. For $D+2 \nu_{S A}=0$, normalization pertains in the limit $\left(\eta_{S A}\right)^{2} \rightarrow 0^{+}$. Regarding mathematics relevant to normalization for $D+2 \nu_{S A}=0$, the delta function that equation (13) shows pertains. Here, $x^{2}$ correlates with $r^{2}$ and $4 \epsilon$ correlates with $\left(\eta_{S A}\right)^{2}$. (Reference [3] provides equation (13).) The difference in domains, between $-\infty<x<\infty$ and equation (5), is not material here. (Our use of this type of modeling features normalization. Considering normalization leads to de-emphasizing possible concerns, regarding singularities as $r$ approaches zero, regarding some $\Psi(r)$.)

$$
\begin{gathered}
D+2 \nu_{S A} \geq 0 \\
\delta(x)=\lim _{\epsilon \rightarrow 0^{+}}(1 /(2 \sqrt{\pi \epsilon})) e^{-x^{2} /(4 \epsilon)}
\end{gathered}
$$

We use the one-element term volume-like to describe solutions for which $D+2 \nu_{S A}>0$. The term volume-like pertains regarding behavior with respect to the coordinate or coordinates that underlie modeling. (For ongoing modeling, generally, the word coordinates - as in $r$ plus angular coordinates - can be appropriate.) We use the one-element term point-like to describe solutions for which $D+2 \nu_{S A}=0$. Here, $\Psi(r)$ is effectively zero for all $r>0$. The term point-like pertains regarding behavior with respect to the coordinate or coordinates that underlie modeling.

We explore some relationships regarding and between solutions.

We explore modeling regarding cases for which $\nu_{S A}$ is not necessarily an integer, $j$ is an integer, and $j \nu_{S A}$ is an integer. We develop a process for transforming fractional-integer- $\nu_{S A}$ modeling into integer$\nu_{S A}$ modeling. We anticipate using such modeling for cases for which $D+2 \nu_{S A} \geq 0, j=2$, and $j \nu_{S A}$ 
Table 9: Some results of recursive applications of equation 15 , assuming that $j=2$

\begin{tabular}{rrrrrl}
\hline$D_{1}$ & $D_{2}$ & $D_{3}$ & $D_{4}$ & $D_{5}$ & $D \ldots$ \\
\hline$\cdots$ & & & & & \\
-1 & -4 & -10 & -22 & -46 & $\ldots$ \\
0 & -2 & -6 & -14 & -30 & $\ldots$ \\
1 & 0 & $\ldots$ & & & Note the case for which $D_{1}=0$. \\
2 & 2 & $\ldots$ & & & 2 \\
3 & 4 & 6 & 10 & 18 & $\ldots$ \\
4 & $\ldots$ & & & & Note the case for which $D_{2}=4$. \\
5 & 8 & 14 & 26 & 50 & $\ldots$ \\
$\cdots$ & & & & & \\
\hline
\end{tabular}

satisfies one of $j \nu_{S A}=-1$ and $j \nu_{S A}=-3$. (See, for example, table 17.) People might also find interest in, for example, cases for which $j=2, \nu_{S A}>0, j \nu_{S A}$ is an integer, and $\nu_{S A}$ is not an integer. (Ongoing modeling does not necessarily consider cases for which $2 \nu_{S A}$ is a positive integer and $\nu_{S A}$ is not an integer.)

We start with equation (14), which re-expresses equation (8). Equation (15) defines, for integer $k$, $D_{k+1}$ in terms of $D_{k}$. Equation (16) pertains. Equation (16) correlates with an equivalent of equation (8). (Some uses of equation (16) may correlate with, in effect, absorbing the factor - in the rightmost term in the equation - of $j^{-2}$ into the term $\xi_{S A}^{\prime} / 2$.)

$$
\begin{gathered}
\Omega_{S A}=\left(1 / j^{2}\right)\left(j \nu_{S A}\right)\left(\left(j \nu_{S A}+j D_{1}-2 j\right)\right. \\
D_{k+1}=j\left(D_{k}-2\right)+2 \\
\Omega_{S A}=\left(1 / j^{2}\right)\left(j \nu_{S A}\right)\left(j \nu_{S A}+\left(j\left(D_{1}-2\right)+2\right)-2\right)=\left(1 / j^{2}\right)\left(j \nu_{S A}\right)\left(j \nu_{S A}+D_{2}-2\right)
\end{gathered}
$$

Adding the assumption that $D_{2}>0$ yields equation (17).

$$
D_{1}>2(1-(1 / j))
$$

Adding the assumptions that $D_{1}$ is an integer and that $j>0$ yields equation $(18)$.

$$
D_{1} \geq 2
$$

For the case $j=2$, equation $(19)$ pertains for $D_{1} \geq 2$.

$$
D_{2}=2 D_{1}-2
$$

For the case $j=2$ and $D_{1}=3$, equation 20 pertains.

$$
D_{2}=2 D_{1}-2=4
$$

Table 9 shows, for $j=2$, results $D_{2}$ from applying equation (15) once to some values of $D_{1}$ and results $D_{k}($ for $k>2)$ ) of reapplying equation (15).

We explore modeling that considers angular coordinates for the sub-case for which $D_{1}=3, j=2$, and $\nu_{S A}=1 / 2$. Here, $\nu_{S A}$ is positive and the possibly (that is, for example, for ongoing modeling) so-called total angular momentum $l \hbar$ correlates with $l=\nu_{S A}=1 / 2$. Equation (21) shows the angular factor in $\Psi(r)=\phi(r) Y_{l, m}(\theta, \phi)$. Equations 22) and (23) pertain. In ongoing modeling, people use notions of two-component spinors and four-component spinors to avoid problems to which the non-equality in equation (22) seems to point.

$$
\begin{gathered}
Y_{1 / 2, \pm 1 / 2}(\theta, \phi)=\exp ( \pm i(1 / 2) \phi), \text { for } 0 \leq \phi \leq 2 \pi \\
Y_{l, m}(\theta, 2 \pi)=\exp ( \pm i \pi)=-1 \neq 1=Y_{l, m}(\theta, 0) \\
Y_{l, m}(\theta, j(2 \pi))=Y_{l, m}(\theta, 0)
\end{gathered}
$$


Table 10: Steps to avoid problems to which equation (22) seems to point

\section{Possible steps}

- Use a transformation from $D_{1}=3$ to $D_{2}=4$. (See equation (20).)

- Split a set of four (as in, $D_{2}=4$ ) oscillators into two sets, each consisting of a pair of oscillators.

- Develop appropriate modeling that correlates with at least one of the two sets of a pair of oscillators.

Table 11: A process for transforming a solution that is appropriate for $D_{1}=D$ dimensions into a solution that is appropriate for $D_{2}=D_{S A}^{\prime}$ dimensions

Steps

- Choose values of $\nu_{S A}, \sigma_{S A}, S_{S A}$, and $D_{S A}^{\prime}$.

- Determine (a first value of) $D$ via equation 26) . Let $D_{1}$ denote this value of $D$.

- Embrace the radial dependence of $\Psi(r)$ that equation (6) implies and set any dependence on angular coordinates to a non-zero constant.

- Combine the radial dependence with an angular dependence appropriate to a solution (to equations (3) and (4)) for which (a second value of) $D$ (in equation (4)) satisfies $D=D_{S A}^{\prime}$. Let $D_{2}$ denote this (second) value of $D$. (The value of $D_{2}$ is not necessarily the same as the value of $D_{1}$.)

- Thereby, produce a $\Psi(r)$ that (may have angular dependence and) pertains regarding $D_{S A}^{\prime}$ dimensions.

Table 10 list steps - other than deploying mathematics correlating with spinors - that proposed modeling suggests to avoid problems to which equation (22) seems to point.

We explore some modeling that considers angular coordinates. Regarding equation (8), we explore mathematics for which equation (24) pertains for some choice of $\sigma_{S A}, S_{S A}$, and $D_{S A}^{\prime}$. Equation (25) restates equation (8). Combining equations (24) and (25) yields equation (26).

$$
\begin{gathered}
\Omega_{S A}=\sigma_{S A} S_{S A}\left(S_{S A}+D_{S A}^{\prime}-2\right), \text { for } \sigma_{S A}= \pm 1 \\
D=2-\nu_{S A}+\left(\nu_{S A}\right)^{-1} \Omega_{S A} \\
D=2-\nu_{S A}+\left(\nu_{S A}\right)^{-1} \sigma_{S A} S_{S A}\left(S_{S A}+D_{S A}^{\prime}-2\right)
\end{gathered}
$$

Table 11 shows a process for transforming a solution that is appropriate for $D_{1}=D$ dimensions into a solution that is appropriate for $D_{2}=D_{S A}^{\prime}$ dimensions. (See equation (26).) Here, we deploy some concepts that ongoing modeling embraces. (See table 7.)

We anticipate using PDE modeling that combines TA-side aspects and SA-side aspects. The following equations define the operators $A_{T A}^{P D E}$ and $A_{S A}^{P D E}$. The symbol $\Psi(t, r)$ denotes a solution.

$$
\begin{gathered}
A_{T A}^{P D E} \Psi(t, r)=\xi_{T A} \Psi(t, r)=\left(\xi_{T A}^{\prime} / 2\right)\left(-\left(\eta_{T A}\right)^{2} \nabla_{t}^{2}+\left(\eta_{T A}\right)^{-2} t^{2}\right) \Psi(t, r) \\
\nabla_{t}^{2}=t^{-\left(D_{T A}-1\right)}(\partial / \partial t)\left(t^{D_{T A}-1}\right)(\partial / \partial t)-\Omega_{T A} t^{-2} \\
A_{S A}^{P D E} \Psi(t, r)=\xi_{S A} \Psi(t, r)=\left(\xi_{S A}^{\prime} / 2\right)\left(-\left(\eta_{S A}\right)^{2} \nabla_{r}^{2}+\left(\eta_{S A}\right)^{-2} r^{2}\right) \Psi(t, r) \\
\nabla_{r}^{2}=r^{-\left(D_{S A}-1\right)}(\partial / \partial r)\left(r^{D_{S A}-1}\right)(\partial / \partial r)-\Omega_{S A} r^{-2}
\end{gathered}
$$

For core proposed modeling, we assume that equation (31) pertains.

$$
0=A^{P D E}=A_{T A}^{P D E}-A_{S A}^{P D E}
$$


Table 12: Representations for ALG solutions

(a) Representation showing individual oscillators

\begin{tabular}{cccccccccc}
\hline Side & 0 & 1 & 2 & 3 & 4 & $\ldots$ & 16 & $\ldots$ & 20 \\
\hline TA & $n_{T A 0}$ & $n_{T A 1}$ & $n_{T A 2}$ & $n_{T A 3}$ & $n_{T A 4}$ & $\ldots$ & $n_{T A 16}$ & $\ldots$ & $n_{T A 20}$ \\
SA & $n_{S A 0}$ & $n_{S A 1}$ & $n_{S A 2}$ & $n_{S A 3}$ & $n_{S A 4}$ & $\ldots$ & $n_{S A 16}$ & $\ldots$ & $n_{S A 20}$ \\
\hline
\end{tabular}

(b) Representation featuring pairings of individual oscillators

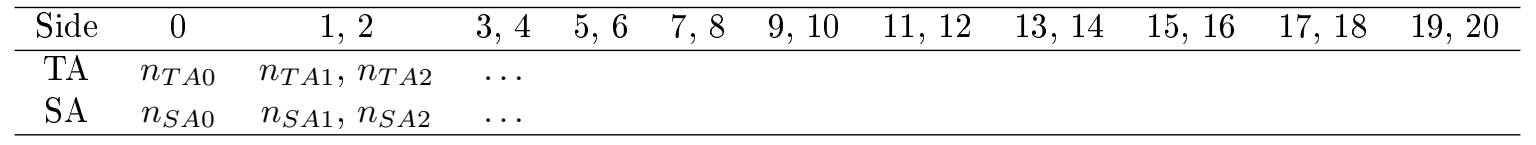

\subsection{ALG mathematics}

We explore mathematics underlying ALG modeling.

Equation 32 shows an ongoing modeling representation for states for a one-dimensional harmonic oscillator. The symbol $\left.\right|_{-}>$correlates with the notion of quantum state. (See equation (2).) Equation (33) shows the ongoing modeling representation for a raising operator. Equation (34) shows the ongoing modeling representation for a lowering operator. In ongoing modeling, $n$ is a nonnegative integer.

$$
\begin{gathered}
\mid n> \\
a^{+}\left|n>=(1+n)^{1 / 2}\right| n+1> \\
a^{-}\left|n>=n^{1 / 2}\right| n-1>
\end{gathered}
$$

Proposed modeling extends the domain correlating with equation (32) from the ongoing modeling domain of $n \geq 0$ to the domain of $n \geq-1$. Proposed modeling includes equations (35) and (36). The extended domain plays roles regarding multidimensional harmonic oscillators.

$$
\begin{aligned}
& a^{+}|-1>=0| 0> \\
& a^{-}|0>=0|-1>
\end{aligned}
$$

Equation (37) correlates with equations (27), (28), 29), and (30). Here, XA can be either one of TA and SA. For each of the two values of XA, $A_{X A}^{A L G}$ includes $D_{X A}$ one-dimensional oscillators.

$$
A_{X A}^{A L G}=\left(\xi_{X A}^{\prime} / 2\right) \sum_{\iota=0}^{D_{X A}-1}-\left(\eta_{X A}\right)^{2}\left(\frac{d}{d r_{X A \iota}}\right)^{2}+\left(\eta_{X A}\right)^{-2}\left(r_{X A \iota}\right)^{2}
$$

For ALG modeling, equation (38) pertains. Each of $A_{T A}^{A L G}$ and $A_{S A}^{A L G}$ correlates with the concept of an isotropic quantum harmonic oscillator. The word isotropic (or, the two-word term equally weighted) also pertains to the pair consisting of $A_{T A}^{A L G}$ and $A_{S A}^{A L G}$. The one-element term double-entry pertains. For example, increasing a TA-side excitation number by one requires either decreasing a different TAside excitation by one or increasing one SA-side excitation by one. The two-element term double-entry bookkeeping pertains.

$$
0=A^{A L G}=A_{T A}^{A L G}-A_{S A}^{A L G}
$$

For core proposed modeling, we assume that equation (38) pertains. Equation (38) provides an ALG analog to PDE equation (31).

Table 12 provides ways to visualize solutions to equation (38). For each of TA and SA, one includes just the columns XA0 through $\mathrm{XA}\left(D_{X A}-1\right)$. Each relevant $n_{X A}$ is an integer. (Note equation (32). Also, for some applications, we assume the following. We assume that equation (38) implies that $\left(\xi_{\text {TA }}^{\prime} / 2\right)=$ $\left(\xi_{S A}^{\prime} / 2\right) \neq 0$. We can assume, without loss of generality, that $\left(\xi_{T A}^{\prime} / 2\right)=\left(\xi_{S A}^{\prime} / 2\right)>0$. Paralleling results that equations (7) and (8) show, we assume, without loss of generality for ALG modeling, that $\left.\eta_{T A}=\eta_{S A}=1.\right)$ 
Equations (38) and (39) characterize all solutions that we include in ALG modeling that is based on isotropic harmonic oscillators.

$$
A_{X A}^{A L G}=\sum_{\iota=0}^{D_{X A}-1}\left(n_{X A \iota}+1 / 2\right)
$$

We posit that equations (40) and (41) extend equation (38). Here, the number, $n$, correlating with excitations satisfies $n \geq 0$.

$$
\begin{aligned}
& a^{+} A_{T A}^{A L G}=a^{+} A_{S A}^{A L G} \\
& a^{-} A_{T A}^{A L G}=a^{-} A_{S A}^{A L G}
\end{aligned}
$$

We posit that equations (42) and (43) have relevance for the domain $-1 \leq n \leq 0$. (See discussion regarding table 44- which pertains for charged leptons - and discussion regarding table 46- which pertains for neutrinos.) This essay de-emphasizes fully discussing implications of applications of equations (42) and (43).

$$
\begin{gathered}
b^{+}\left|n>=n^{1 / 2}\right| n+1> \\
b^{-}\left|n>=(1+n)^{1 / 2}\right| n-1>
\end{gathered}
$$

We discuss symmetries that correlate with mathematics for isotropic harmonic oscillators.

Table 13 shows symbols that this essay suggests and groups to which proposed modeling refers. Regarding information pertaining to groups, aside from rows (in the table) that show a negative contribution to $A_{X A}^{A L G}$ or that show the symbol $\pi_{@_{0}, @_{-1}}$, information in the table comports with standard relationships between mathematics of group theory and mathematics for isotropic quantum harmonic oscillators. The leftmost column shows the relevant number of oscillators. For each row, the symbol $X A$ can be TA, in which case all of the oscillators are TA-side oscillators, or SA, in which case all of the oscillators are SA-side oscillators. The symbol $\chi$ correlates with the concept of choice. The symbol $\chi_{a}$ pertains to one oscillator and correlates with the equation $n_{X A}=a$. The symbol $S 1 G$ denotes a group with one generator. The number of generators for $U(1)$ is two. One generator correlates with integer increases regarding the number of excitations that pertain for the oscillator for which the table shows $n \geq 0$. One generator correlates with integer decreases regarding the number of excitations that pertain for the oscillator for which the table shows $n \geq 0$. The symbol $\pi$ correlates with the concept of permutations. The symbol $\pi_{a, b}$ denotes two possibilities. Regarding the two oscillators, for one possibility, $a$ pertains to the first oscillator and $b$ pertains to the second oscillator. For the other possibility, $a$ pertains to the second oscillator and $b$ pertains to the first oscillator. Regarding the symbol $\pi_{n, @_{-1}}$, the $U(1)$ symmetry correlates with the $n$. Use of the symbol $\pi_{@_{0}, @_{-1}}$ is sensitive to context. In some contexts, a $U(1)$ symmetry pertains and correlates with the appearance of $@_{0}$. In some contexts, no $U(1)$ symmetry pertains. The symbol A0+denotes $\pi_{@_{0}} @_{0}$. The symbol $\kappa$ correlates with the concept of a continuous set of choices. For example, regarding two oscillators XA1 and XA2, equations (44) and (45) describe the continuum of possibilities correlating with $\kappa_{0,-1}$. Here, each of $d$ and $e$ is a complex number. The symbol [blank] denotes the concept that, in tables such as table 35. one can interpret a blank cell as correlating with $\kappa_{0,-1}$. Regarding $S U(j)$, each one of the symbols $\kappa_{@_{-1}, \cdots, @_{-1}}$ and $\kappa_{@_{0}, \cdots, @_{0}}$ correlates with a continuous set of choices involving amplitudes pertaining to $j$ oscillators. The number of generators for $S U(j)$ is $j^{2}-1$.

$$
\begin{gathered}
d\left|n_{X A 1}=0, n_{X A 2}=-1>+e\right| n_{X A 1}=-1, n_{X A 2}=0> \\
|d|^{2}+|e|^{2}=1
\end{gathered}
$$

We discuss relationships between the numbers of generators for some $S U(j)$ groups.

In equation (46), $g_{j}$ denotes the number of generators of the group $S U(j)$, the symbol $\mid$ denotes the word divides (or, the two-word phrase divides evenly), and the symbol $X$ denotes the four-word phrase does not divide evenly. For some aspects of proposed modeling, equation (46) correlates with ending the series $S U(3), S U(5), \cdots$ at the item $S U(7)$. For some aspects of proposed modeling, the series $S U(3)$, $S U(5), S U(7)$, and $S U(17)$ might pertain. 
Table 13: Number of oscillators, symbols, groups, and contributions to $A_{X A}^{A L G}$

\begin{tabular}{ccccc}
\hline Oscillators & Symbol(s) & Group & Generators & Contribution to $A_{X A}^{A L G}$ \\
\hline 1 & $\chi_{0}$ & $S 1 G$ & 1 & $1 / 2$ \\
1 & $@_{0}$ & - & - & $1 / 2$ \\
1 & $@_{-1}$ & - & - & $-1 / 2$ \\
1 & $n{ }_{-}(n \geq 0)$ & $U(1)$ & 2 & $n-(1 / 2)$ \\
2 & $\pi_{n, @_{-1}(n \geq 0)}$ & $U(1)$ & 2 & $n$ \\
2 & $\pi_{@_{0}, @_{-1}}$ & $U(1)$ & 2 & 0 \\
2 & $\pi_{@_{0}, @_{-1}}$ & - & - & 0 \\
2 & $\mathrm{~A} 0+, \pi_{@_{0}, @_{0}}$ & - & - & -1 \\
2 & $\pi_{@_{-1}, @_{-1}}$ & - & & 0 \\
2 & {$\left[\mathrm{blank},, \kappa_{0,-1}\right.$} & - & - & $j / 2$ \\
$j$ & $\kappa_{@_{0}, \cdots, @_{0}}$ & $S U(j), j \geq 2$ & $j^{2}-1$ & $-j / 2$ \\
$j$ & $\kappa_{@_{-1}, \cdots, @_{-1}}$ & $S U(j), j \geq 2$ & $j^{2}-1$ & -1 \\
\hline
\end{tabular}

Table 14: Some aspects and objectives of physics modeling regarding an object

Aspect and objective

- Description - Describe the object.

- Motion - Regarding the object, describe a trajectory (classical physics) or wave function (quantum physics).

- Measurements - Regarding an observer's measurements of the object, predict or explain measured values.

$\left.g_{3}\left|g_{5}, g_{3}\right| g_{7}, g_{5}\left|g_{7} \quad g_{5} \backslash g_{9}, g_{7}\right\rangle g_{9}, g_{7}\right\rangle\left\langle g_{11} \quad g_{3}\left|g_{17}, g_{5}\right| g_{17}, g_{7}\right| g_{17}$

We anticipate invoking the mathematical notion of ending a series $S U(3), S U(5), \cdots$ at the item $S U(7)$. Sometimes, we correlate an ending with physics data. Sometimes, we correlate an ending with symmetries related to kinematics conservation laws.

We note a relationship between $S U(j)$ groups and the group $U(1)$.

Equation (47) echoes mathematics and some ongoing modeling. Here, each of the positive integers $j_{1}$ and $j_{2}$ is at least two. The symbol $\supset$ correlates with the notion that each group to the right of the symbol is a subgroup of the group to the left of the symbol.

$$
S U\left(j_{1}+j_{2}\right) \supset S U\left(j_{1}\right) \times S U\left(j_{2}\right) \times U(1)
$$

\subsection{Relationships between applications of various types of models}

Table 14 shows some aspects and objectives of physics modeling regarding an object. (This essay de-emphasizes discussing many notions regarding the completeness with which modeling does or could meet objectives that table 14 notes. For example, we do not necessarily adequately discuss the extent to which modeling regarding objects leads to modeling that sub-optimally describes nature. For example, we do not necessarily adequately discuss the extent to which models completely separate one object from the rest of the universe. Ongoing modeling devotes much attention to the notion of entanglement. For example, we do not necessarily adequately discuss the notion of how well modeling defines objects. In ongoing modeling, modeling based on the Dirac equation does not necessarily completely separate an electron from a correlated positron. And so forth.)

This essay seeks to develop core proposed modeling - that models descriptions of objects - that complements core ongoing modeling models regarding motion and measurements.

Table 15 discusses the terms KMS (or, kinematics modeling space), PFS (or, particle field space), and UMS (or, united modeling space).

Table 16 discusses uses for core proposed modeling ALG models.

Table 17 characterizes applications - that this essay discusses - of various types of modeling based on harmonic oscillator mathematics. For each of the cases PFS PDE and KMS PDE, this essay uses symbols such as $t$ and $r$ to denote relevant coordinates. PFS PDE use of such a symbol does not necessarily completely correlate with KMS PDE use of the same symbol.

UMS modeling provides a basis to explore relationships between KMS modeling and core proposed modeling PFS models. This essay does not fully explore relationships between KMS modeling and core 
Table 15: KMS (or, kinematics modeling space), PFS (or, particle field space), and UMS (or, united modeling space)

\begin{tabular}{l}
\hline Aspect \\
- The term KMS abbreviates the three-word term kinematics modeling space. The term KMS \\
refers to modeling that can - and, in ongoing modeling, often does - use coordinates that people \\
use to model aspects that people correlate with notions of space-time. Ongoing modeling tends to \\
use KMS modeling to address the topics of motion and measurements. (See table 14.) Core \\
proposed modeling tends to embrace results from such ongoing modeling. Supplementary proposed \\
modeling suggests new KMS modeling that might supplement such ongoing modeling. \\
- The term PFS abbreviates the three-word term particle field space. The term PFS refers to \\
modeling that generally does not correlate directly with space-time coordinates. Core proposed \\
modeling tends to use PFS modeling to address the topic of objects (especially, elementary \\
particles). (See table 14.) Core ongoing modeling seems not to include, for at least elementary \\
particles, an analog to PFS modeling. \\
- The term UMS abbreviates the three-word term united modeling space. The term UMS refers to \\
modeling that embraces aspects that correlate with, at least, KMS modeling and PFS modeling. \\
Core proposed modeling tends to use UMS ALG modeling to address topics regarding properties of \\
objects and regarding conservation laws. Here, the two-word term conservation laws pertains \\
regarding internal properties of objects and regarding motions of objects. (Perhaps, note table 14.) \\
Unverified ongoing modeling seems to suggest - for example via the notion of a so-called theory of \\
everything - possible desirability for people to explore notions such as UMS modeling.
\end{tabular}

Table 16: Uses for core proposed modeling ALG models

\begin{tabular}{l}
\hline Use \\
- Core proposed modeling ALG models provide means to extend and catalog results - regarding \\
elementary particles - that core proposed modeling PDE models output. \\
- Core proposed modeling ALG models suggest means to integrate modeling regarding the aspects \\
that table 14 discusses.
\end{tabular}

Table 17: Applications of various types of modeling based on harmonic oscillator mathematics

\begin{tabular}{lccc}
\hline Type & Applications & $\begin{array}{c}\text { Integer 2 } \\
\text { (pertains re PDE) }\end{array}$ & $\begin{array}{c}\text { Integer } n \\
\text { (pertains re ALG) }\end{array}$ \\
\hline Core proposed modeling & PFS & $2 \nu<0$ & $n \geq-1$ \\
Core ongoing modeling & KMS & $2 \nu \geq 0$, even & $n \geq 0$ \\
Unverified ongoing modeling & KMS & $2 \nu \geq 0$, even & $n \geq 0$ \\
Supplementary proposed modeling & KMS & $2 \nu \geq 0$, even & $n \geq 0$ \\
Core proposed modeling ALG models & UMS & - & $0 \geq n \geq-1$ \\
\hline
\end{tabular}


proposed modeling PFS models. We suggest that some such exploration might feature linking PFS modeling and KMS modeling via modeling that involves concepts such as phase spaces, tangent spaces, or symplectic geometry. (See, for example, reference [4.) One concept might include the notion of considering that PFS PDE modeling might pertain mathematically to a tangent space to a space that correlates with space-time coordinates. One concept might include the notion of considering that PFS PDE modeling might pertain mathematically to a tangent space to a space that correlates with a phase space that correlates with an energy-momentum space and with space-time coordinates. Per table 17, we suggest that people might use core proposed modeling ALG models as bases for exploring such concepts.

We provide additional perspective about modeling that this essay discusses.

Generally, core proposed modeling embraces motion via representations for motion-centric conservation laws and via relying on ongoing modeling models for motion. Hence, core proposed modeling uses, at least indirectly, KMS modeling. Core proposed modeling uses PFS modeling to, for example, match known and predict new elementary particles. Supplementary proposed modeling suggests uses of KMS modeling to, for example, model aspects of multicomponent objects.

KMS PDE modeling can feature linear coordinates or radial plus angular coordinates. PFS PDE modeling features radial coordinates. Each of ongoing modeling and proposed modeling uses modeling for which solutions that correlate with equation (1) normalize. Each normalized PFS $\nu<0$ solution normalizes because the number of dimensions is adequately large.

Proposed modeling associates the one-element term TA-side with modeling that correlates with the two-word term temporal aspects. The two-word term temporal aspects echoes notions of temporal aspects of ongoing modeling KMS modeling that uses space-time coordinates. We use the term temporal aspects in the context of PFS modeling and in the context of KMS modeling. Proposed modeling associates the one-element term SA-side with modeling that correlates with the two-word term spatial aspects. The two-word term spatial aspects echoes notions of spatial aspects of ongoing modeling KMS modeling that uses space-time coordinates. We use the term spatial aspects in the context of PFS modeling and in the context of KMS modeling.

\subsection{A method that organizes properties of elementary particles and other objects}

We discuss a method to organize and conceptualize aspects of modeling that we develop below. The method itself might point to insight about modeling and about nature. (See discussion related to table 111.) However, for the moment, we treat the method as, in essence, an organizational convenience.

The method has bases in the notion that $S U(17)$ symmetry might be relevant. The method has bases in the notion that modeling based on 17 harmonic oscillators can correlate with $S U(17)$ symmetry. (See table 13.)

The method has bases in table 12b. We label the 17 oscillators as HO1, HO2, ..., and HO17.

The method uses equation (47).

The method correlates with the two one-element terms UMS and ALG.

Table 18 notes aspects pertaining to some types of symmetries and related conservation laws. Proposed modeling correlates these aspects with aspects regarding interactions between objects. The terms exact symmetry, approximate symmetry, and no symmetry correlate with mathematical modeling and with aspects of ongoing modeling. The following discussion reprises examples that table 18 shows. An electron, correlates with an exact symmetry regarding conservation of charge. An electron correlates with a rebuttable always conservation law that correlates with charge. For an interaction between the electron and other objects that all correlate with rebuttable always conservation of charge, the outgoing fermion that correlates with the incoming electron has the same charge as the electron. For that example, a notion of always conservation of charge - across the electron and the related outgoing fermion - pertains. Suppose, however, that one of the incoming particles is a $\mathrm{W}$ boson. The $\mathrm{W}$ boson correlates with no symmetry and with not necessarily conservation of charge. If the $\mathrm{W}$ boson is a positively charged $\mathrm{W}$ boson, the interaction can transform the electron into a neutrino, which has zero charge. Here, in effect, the not necessarily conservation of charge correlating with the $\mathrm{W}$ boson rebuts the rebuttable always conservation of charge for the electron.

Table 19 discusses aspects relevant to the method to organize properties of objects.

Table 20 discusses the method and previews aspects of applications of the method.

Table 21 posits aspects - of proposed modeling and of nature - that might correlate with SM6a and SM6b symmetries.

\section{Results: elementary particles}

This unit predicts elementary particles that people have yet to find. 
Table 18: Aspects pertaining to some types of symmetries and related conservation laws

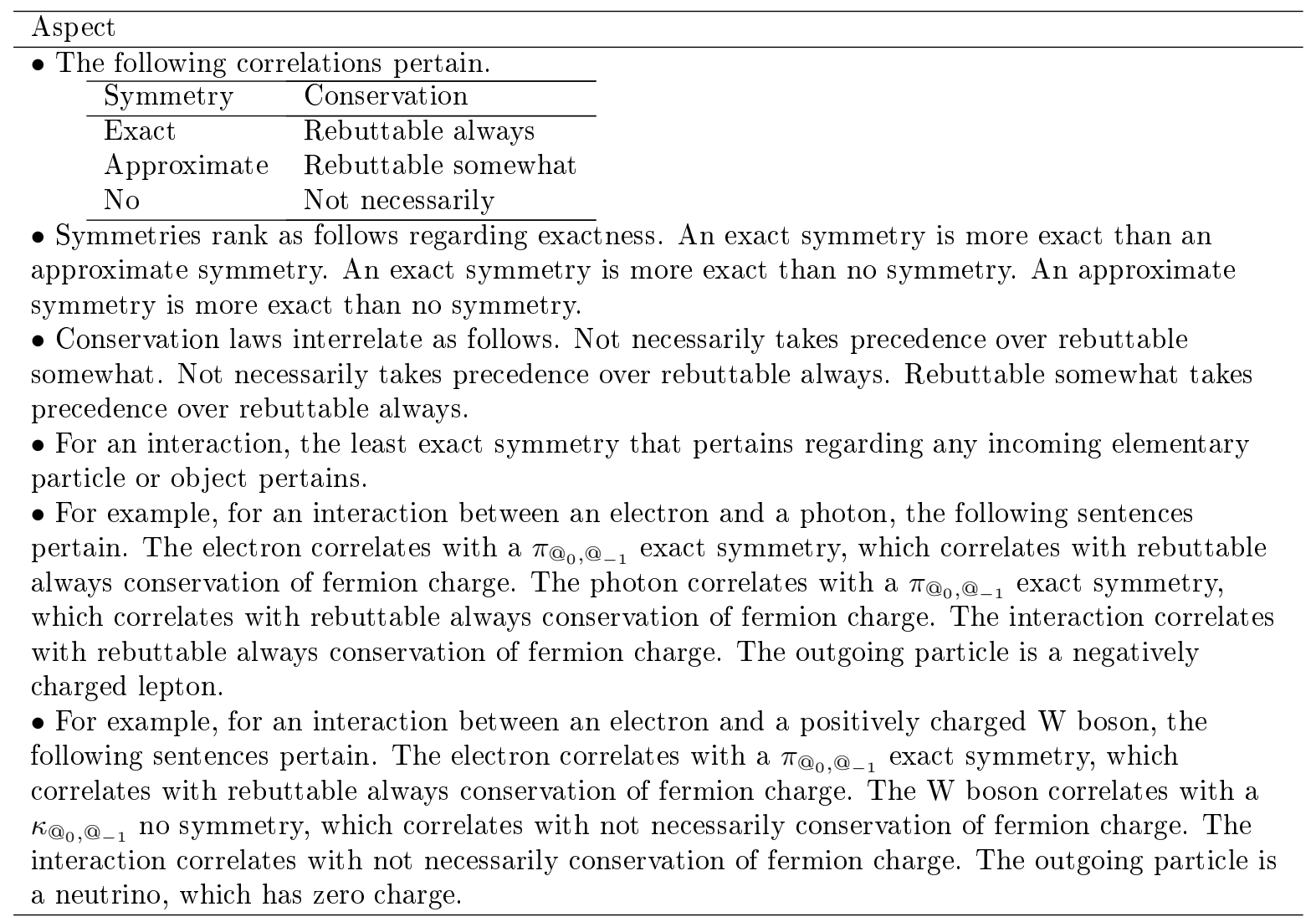

Table 19: Aspects relevant to a method to organize properties of objects

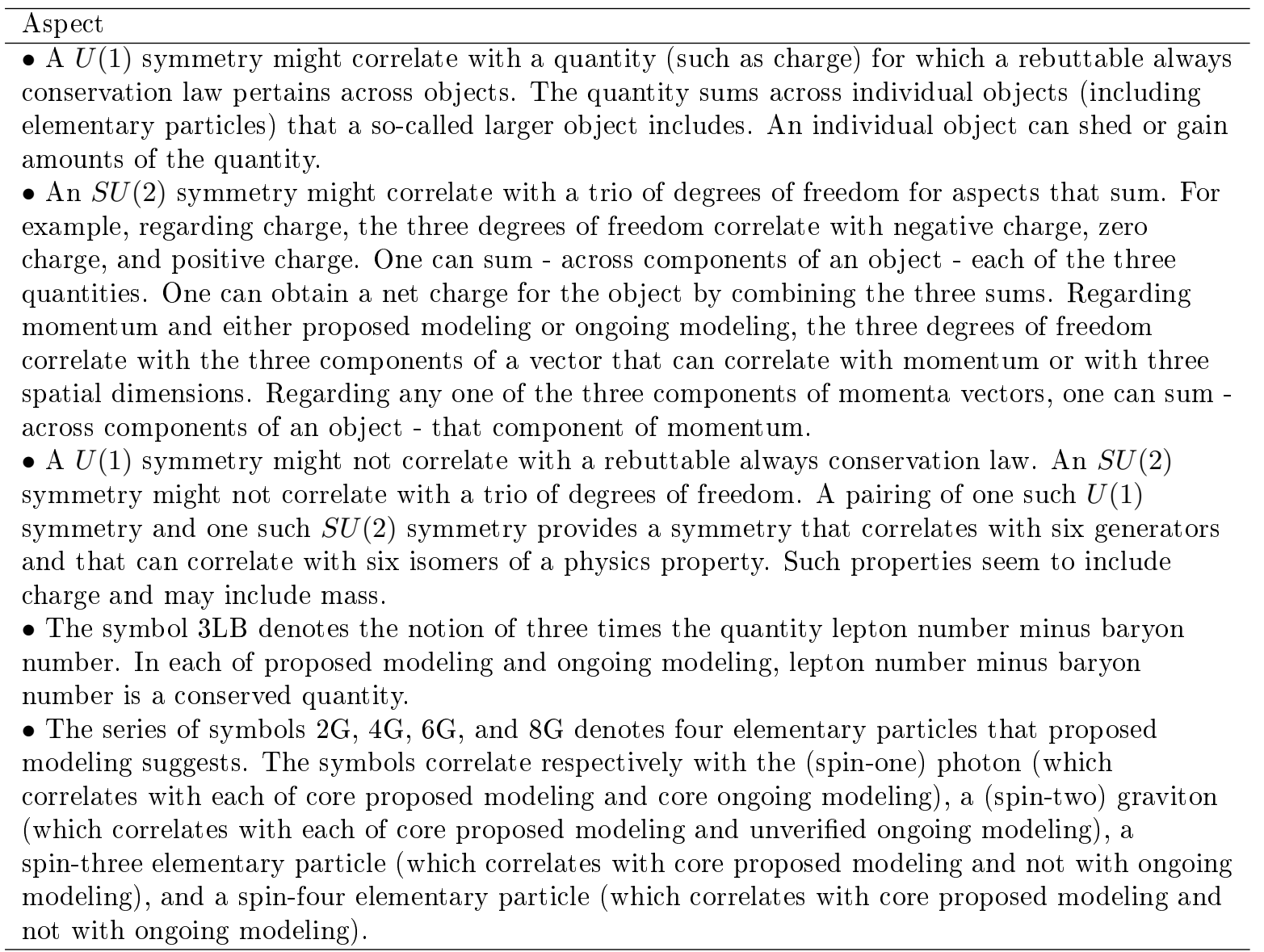


Table 20: A method to organize properties of objects (with the one-element term HO abbreviating the two-word phrase harmonic oscillator)

(a) Steps that develop the method (with the word generations pertaining to elementary fermions)

\begin{tabular}{cccclll}
\hline HO range & $j_{1}+j_{2}$ & $j_{1}$ & $j_{2}$ & HO range re $j_{1}$ & HO range re $j_{2}$ & Use of $U(1)$ \\
\hline $1-17$ & 17 & 8 & 9 & $1-8$ (deferred) & $9-17$ (deferred) & (deferred to 1-2) \\
$1-8$ & 8 & 4 & 4 & $1-2 ; 7-8$ (deferred) & 3-6 (deferred) & Cons of energy \\
$1-2 ; 7-8$ & 4 & 2 & 2 & $1-2$ Cons of charge & 7-8 Cons of 3LB & Cons of 3LB \\
$3-6$ & 4 & 2 & 2 & $3-4$ Mass, SM6a $S U(2)$ & $5-6$ Generations & 3-4 SM6a $U(1)$ \\
$9-17$ & 9 & 5 & 4 & $9-10 ; 15-17$ (deferred) & 11-14 (deferred) & (deferred to 11-12) \\
$9-10 ; 15-17$ & 5 & 3 & 2 & $9-10 ; 17$ SI $S U(3)$ & $15-16$ SM6b $S U(2)$ & $15-16$ SM6b $U(1)$ \\
$11-14$ & 4 & 2 & 2 & $11-12$ Cons of ang mom & $13-14$ Cons of mom & Cons of mom \\
\hline
\end{tabular}

(b) Some notes

Note

- Each one-word item (deferred) implies that a later row in table 20a pertains for the HO range.

- Each multi-element item (deferred to $k_{1}-k_{2}$ ) implies that a later row discusses the HO range

$k_{1}-k_{2}$ and that the $U(1)$ symmetry pertains to the $k_{1}-k_{2}$ HO range.

- The multi-element item 1-2; 7-8 denotes the four oscillators $1,2,7$, and 8 .

- Cons denotes the word conservation.

- Regarding the item 3-4 Mass, a property pertaining to all objects is rest mass (or - equivalently rest energy).

- The items 3-4 SM6a $S U(2)$ and 3-4 SM6a $U(1)$ correlate with the six generators of an $S U(2) \times U(1)$ symmetry.

- Regarding the item 5-6 Generations, the property pertaining to all objects is freeable energy (or, energy above ground state).

- Conservation of energy - for an individual object - correlates with conservation of rest energy (or, rest mass times $c^{2}$ - in which $c$ denotes the speed of light) minus freeable energy.

- The multi-element item 9-10; 15-17 denotes the five oscillators 9, 10, 15, 16, and 17 .

- The SI $S U(3)$ item correlates with strong interaction $S U(3)$ symmetry.

- The items 15-16 SM6b $S U(2)$ and 15-16 SM6b $U(1)$ correlate with the six generators of an $S U(2) \times U(1)$ symmetry.

- The two-element abbreviation ang mom abbreviates the two-word phrase angular momentum.

- The one-element abbreviation mom abbreviates the word momentum.

(c) Additional notes

Note

- In table 20a, HO range 1-2 correlates with charge and with the notion that $2 \mathrm{G}$ interacts with charge.

- In table $20 \mathrm{a}, \mathrm{HO}$ range $3-4$ correlates with rest energy and with the notion that $4 \mathrm{G}$ interacts with rest energy.

- In table 20a, HO range 5-6 correlates with freeable energy and with the notion that $6 \mathrm{G}$ interacts with freeable energy.

- Regarding elementary particles, the $S U(2)$ that correlates with SM6a correlates with three generations for elementary fermions. (See table 58a.) This notion correlates with a seemingly approximate - but not exact - symmetry that pertains regarding elementary leptons. (See discussion regarding table 96 .)

- In table $20 \mathrm{a}, \mathrm{HO}$ range $7-8$ correlates with $3 \mathrm{LB}$ and with the notion that $8 \mathrm{G}$ interacts with $3 \mathrm{LB}$.

- The two-element term G-family physics includes $2 \mathrm{G}, 4 \mathrm{G}, 6 \mathrm{G}$, and $8 \mathrm{G}$. The term G-family physics does not include, for example, the bosons associated with the weak interaction.

- In table 20a, assignments of oscillators in the HO range 9-17 do not necessarily reflect G-family physics. People might explore possibilities for developing principles governing such assignments. Without such principles, people might consider that proposed modeling is invariant under permutation of the assignments. 
Table 21: Aspects - of proposed modeling and of nature - that might correlate with SM6a and SM6b symmetries

(a) Aspects

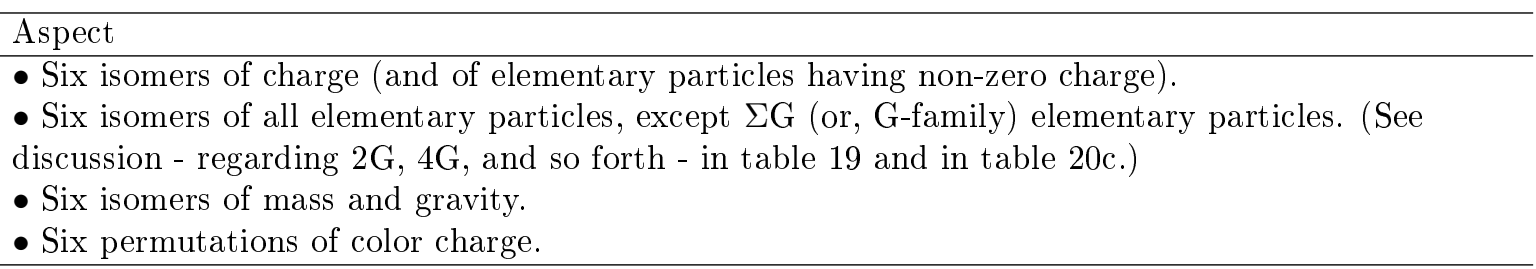

(b) Notes

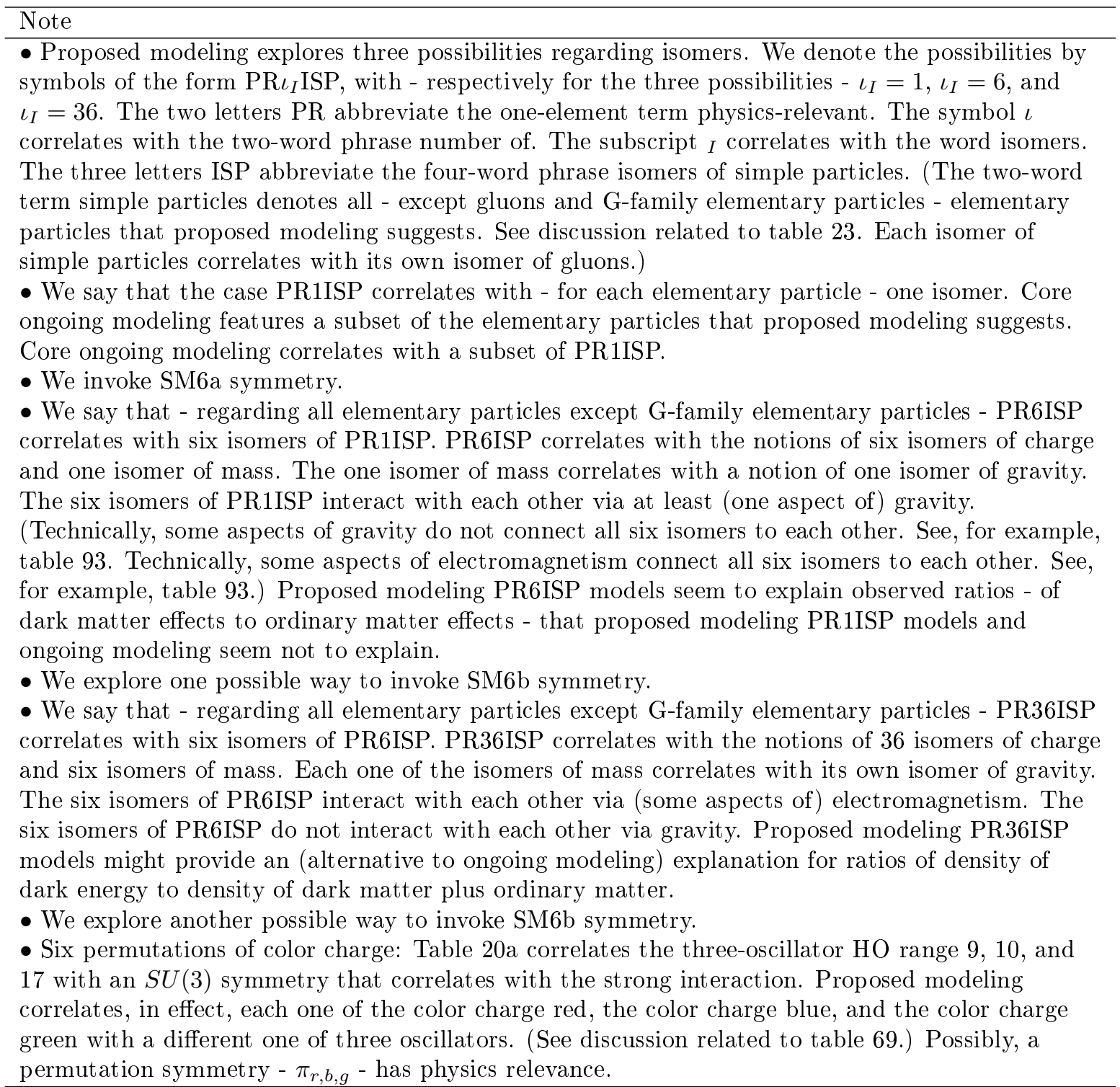


Table 22: Use of the two-word term elementary particle

(a) Vocabulary regarding aspects that people might consider as settled

Note

- This essay de-emphasizes the notion that each quark correlates, based on the existence of three color charges, with three elementary particles.

(b) Aspects that are not necessarily universal throughout proposed modeling and ongoing modeling

\begin{tabular}{l}
\hline Aspect \\
- Proposed modeling suggests some modeling that correlates aspects of the residual strong force \\
with elementary particles and not necessarily with ongoing modeling notions that do not directly \\
involve elementary particles. Examples include the following. Proposed modeling based on \\
so-called 2J bosons (or, jay elementary particles) might correlate with ongoing modeling that \\
correlates with the so-called Pauli exclusion force and a repulsive component of the residual strong \\
force. Proposed modeling based on a so-called 0P boson (or, pie elementary particle) might \\
correlate with ongoing modeling that correlates with virtual pions, a Yukawa potential, and an \\
attractive component of the residual strong force. \\
- Proposed modeling suggests some new modeling that people might correlate with ongoing \\
modeling notions that correlate with terms such as the five-word term interactions with the \\
quantum vacuum. Examples include some interactions involving the so-called 0I boson (or, aye \\
elementary particle) and some interactions involving the $2 \mathrm{~J}$ bosons.
\end{tabular}

(c) Policy regarding using the two-word term elementary particle

Aspect

- This essay uses the two-word term elementary particle so as to include proposed modeling aspects - such as the 0I, 0P, and 2J bosons - to which table 22b alludes.

\subsection{Summary: a table of known and suggested elementary particles}

We suggest that the notion of elementary particle might depend, to some extent, on modeling and vocabulary that people choose to use.

Table 22 discusses use - in this essay - of the two-word term elementary particle. Table 22a discusses vocabulary regarding aspects that people might consider as settled. Table $22 \mathrm{~b}$ discusses aspects that are not necessarily universal throughout proposed modeling and ongoing modeling. Aspects that table $22 \mathrm{~b}$ mentions correlate directly with possible elementary bosons and do not necessarily correlate directly with possible elementary fermions. Table 22c states policy that this essay follows regarding using the two-word term elementary particle.

Table 23 catalogs elementary particles that core ongoing modeling recognizes or that core proposed modeling suggests. (Core proposed modeling includes all of the elementary particles that core ongoing modeling recognizes.) Each row in the table 23 a features one value of spin $S$. The symbol $S$ denotes spin, in units of $\hbar$. (Technically, $S$ correlates with the $S$ in the ongoing modeling expression $S(S+1) \hbar^{2}$.) The definition $\Sigma=2 S$ provides for numbers $\Sigma$ that are non-negative integers. The value of $\Sigma$ appears as the first element of each two-element symbol $\Sigma \Phi$. The letter value of $\Phi$ denotes a so-called family of elementary particles. The symbol $\Sigma \Phi$ denotes a so-called subfamily of elementary particles. The expression $m \doteq 0$ denotes a notion of zerolike mass. Some ongoing modeling models correlate neutrinos with small positive masses. Some ongoing modeling models correlate neutrinos with zero masses. The expression $m>0$ correlates with mass being positive in all ongoing modeling models and in all proposed modeling models. A number $(n)$ denotes a number of elementary particles. A number $((n))$ denotes a number of modes. Table $23 \mathrm{~b}$ provides additional information regarding items that table 23a lists. Table $26 \mathrm{c}$ alludes to possible candidate elementary particles that table 23 does not include and that this essay de-emphasizes. For some elementary particles that people have yet to find, table $23 \mathrm{~b}$ shows names that this essay suggests. Regarding $\Sigma_{\max }$, see discussion regarding equation (48) and equation (49).

We use the two-word term simple particle to pertain to each entry in table 23 other than G-family entries and U-family entries. We correlate the two-word term root force with each G-family entry in table 23 and with the U-family entry in table 23. This use of the word root reflects the notion that some ongoing modeling PDE mathematics-based modeling, which has bases in ongoing modeling KMS aspects of root forces, outputs solutions that correlate with known and suggested simple particles. (See discussion related to equation (58). This essay does not necessarily suggest physics meaning for such 
Table 23: Elementary particles (or simple particles and root forces)

(a) Simple particles and root forces (with notation featuring names of families)

\begin{tabular}{cccc}
\hline Spin $(S)$ & $\Sigma$ & $m>0$ & $m \doteq 0$ \\
\hline 0 & 0 & $0 \mathrm{H}(1), 0 \mathrm{P}(1)$ & $0 \mathrm{I}(1)$ \\
$1 / 2$ & 1 & $1 \mathrm{C}(3), 1 \mathrm{Q}(6)$ & $1 \mathrm{~N}(3), 1 \mathrm{R}(6)$ \\
1 & 2 & $2 \mathrm{~W}(2), 2 \mathrm{~T}(4)$ & $2 \mathrm{~J}(3)$ \\
1 & 2 & & $2 \mathrm{G}((2)), 2 \mathrm{U}(8)$ \\
2 & 4 & & $4 \mathrm{G}((2))$ \\
3 & 6 & & $6 \mathrm{G}((2))$ \\
$\ldots$ & $\ldots$ & & $\Sigma_{\max } \mathrm{G}((2))$ \\
$\Sigma_{\max } / 2$ & $\Sigma_{\max }$ & & \\
\hline
\end{tabular}

(b) Simple particles and root forces (with notation featuring names of elementary particles; with * denoting that people might have yet to find the elementary particles; and with TBD denoting the three-word phrase to be determined)

\begin{tabular}{cccc}
\hline Spin $(S)$ & $\Sigma$ & $m>0$ & $m \doteq 0$ \\
\hline 0 & 0 & Higgs boson (1), Pie* (1) & Aye* $^{*}(1)$ \\
$1 / 2$ & 1 & Charged leptons (3), Quarks (6) & Neutrinos (3), Arcs* $(6)$ \\
1 & 2 & Z and W bosons (2), Tweaks* (4) & Jays* $(3)$ \\
1 & 2 & & Photon $((2))$, Gluons $(8)$ \\
2 & 4 & Graviton* $((2))$ \\
3 & 6 & TBD* $\left.^{*}(2)\right)$ \\
$\ldots$ & $\ldots$ & $\ldots$ \\
$\Sigma_{\max } / 2$ & $\Sigma_{\max }$ & $\mathrm{TBD}^{*}((2))$ \\
\hline
\end{tabular}

use of the word root. Beyond the modeling-based contrast with the term root force, this essay does not necessarily suggest physics meaning for such use of the word simple.) We correlate the three-element term long-range root forces with the $\mathrm{G}$ family.

Particle counts in table 23 de-emphasize modeling that would count, for example, a down quark with green color charge as differing from a down quark with red color charge. (See table 22a.)

We discuss the elementary particles for which the spin is zero (or, $\Sigma=0$ ).

The $0 \mathrm{H}$ particle is the Higgs boson.

The $0 \mathrm{P}$, or so-called pie, possible particle would correlate with a core ongoing modeling notion of an attractive component of the residual strong force.

The 0I, or so-called aye, particle is a possible zerolike-mass relative of the Higgs boson. Proposed modeling suggests that the aye particle is a candidate for the ongoing modeling notion of an inflaton.

We discuss the elementary particles for which the spin is one-half (or, $\Sigma=1$ ).

The three $1 \mathrm{C}$ particles are the three charged leptons - the electron, the muon, and the tauon.

The six 1Q particles are the six quarks.

The three $1 \mathrm{~N}$ particles are the three neutrinos. Some aspects of ongoing modeling suggest that at least one neutrino mass must be positive. At least one positive mass might explain neutrino oscillations and some astrophysics data. Some aspects of ongoing modeling, such as some aspects of the Standard Model, suggest that all neutrino masses are zero. Proposed modeling suggests that effects of $8 \mathrm{G}$ forces might fully explain neutrino oscillations and the relevant astrophysics data. For example, proposed modeling suggests that $8 \mathrm{G}$ forces lead to effects that ongoing astrophysics modeling would correlate with a sum of neutrino masses of $3 \alpha^{2} m_{\epsilon}$. The symbol $\alpha$ denotes the fine-structure constant. The symbol $m_{\epsilon}$ denotes the mass of an electron. The amount $3 \alpha^{2} m_{\epsilon}$ falls within the range that ongoing astrophysics modeling attributes to observed data. (See equations $(142)$ and 1143$)$.) The $8 \mathrm{G}$ forces do not interact with the property of mass. Proposed modeling suggests the possibilities that each neutrino has zero mass or that neutrinos have non-zero (zerolike) masses that are significantly smaller than $\alpha^{2} m_{\epsilon}$.

The six 1R, or so-called arc, possible particles are zero-charge zerolike-mass analogs of the six quarks. Hadron-like particles made from arcs and gluons contain no charged particles and measure as dark matter.

We discuss the elementary particles, other than G-family elementary particles, for which the spin is one (or, $\Sigma=2$ ).

The two $2 \mathrm{~W}$ particles are the two weak interaction bosons - the $\mathrm{Z}$ boson and the $\mathrm{W}$ boson. 
The four 2T, or so-called tweak, possible particles are analogs to the weak interaction bosons. The charge of one non-zero-charge $2 \mathrm{~T}$ particle is two-thirds the charge of the $\mathrm{W}$ boson. The charge of one non-zero-charge $2 \mathrm{~T}$ particle is one-third the charge of the $\mathrm{W}$ boson. The non-zero-charge tweak particles may have played roles in the creation of baryon asymmetry. The non-zero charge tweak particles might correlate with unverified ongoing modeling notions of leptoquarks.

The 2J particles, or so-called jays, are possible zerolike-mass bosons. The jay particles would correlate with a core ongoing modeling notion of a repulsive component of the residual strong force. (The jay particles would correlate with a core ongoing modeling notion of a Pauli exclusion force.) Proposed modeling suggests that the jay particles played roles that ongoing modeling correlates with times just before the inflationary epic and with times during the inflationary epoch.

The eight $2 \mathrm{U}$ particles are the eight gluons. In each of core ongoing modeling and core proposed modeling, gluons correlate with the strong interaction and bind quarks into hadrons. Proposed modeling suggests that gluons bind arcs into hadron-like particles.

We discuss additional roles for the aye boson and jay bosons.

Some proposed modeling models correlating with the aye particle and the jay particles might correlate with some ongoing modeling models that include notions of interactions with a quantum vacuum.

We discuss G-family forces.

Each G-family force exhibits two modes. Our discussion tends to focus on circularly polarized modes. One mode correlates with left circular polarization. One mode correlates with right circular polarization. For 2G, ongoing modeling suggests classical physics models and quantum physics models. The word electromagnetism can pertain. Proposed modeling suggests modeling that provides for $2 \mathrm{G}$ aspects that include and complement ongoing modeling electromagnetism. Regarding gravitation, ongoing modeling suggests classical physics models. Proposed modeling suggests modeling for $4 \mathrm{G}$ aspects that include and complement ongoing modeling gravitation. Proposed modeling regarding $4 \mathrm{G}$ includes classical physics aspects and quantum physics aspects. Proposed modeling regarding $4 \mathrm{G}$ includes aspects that ongoing modeling correlates with the four-word term dark energy negative pressure. Proposed modeling suggests that quantum interactions, involving simple fermions, mediated by $4 \mathrm{G}$ can correlate with a notion of rebuttable somewhat conservation of fermion generation. Ongoing modeling does not include 6G aspects and does not include $8 \mathrm{G}$ aspects. Proposed modeling suggests that $8 \mathrm{G}$ interacts with lepton number minus baryon number.

Regarding G-family forces, proposed modeling suggests, in some sense, more than one component for each one of some $\Sigma \mathrm{G}$. For example, $2 \mathrm{G}$ includes one component that correlates with interactions with charge and one component that correlates with interactions with nominal magnetic dipole moment. This notion of components is appropriate because aspects of proposed modeling can address the topics of properties and interactions without necessarily selecting an ongoing modeling model for motion. (See, for example, discussion regarding table 61 and discussion regarding table 62) The notion of components is essential for proposed modeling models that suggest explanations for observed ratios of dark matter amounts (or other effects) to ordinary matter amounts (or other effects). (See discussion regarding table 89 and discussion regarding tables 92 and 93.)

Proposed modeling suggests that one of equation (48) and equation (49) pertains. Proposed modeling includes the possibility that nature includes 10G, 12G, 14G, 16G, 18G, and 20G bosons. (See discussion related to table 48 and discussion related to equation (135).) G-family bosons for which $\Sigma \geq 10$ would interact with anomalous properties and not with nominal properties. Examples of nominal properties include charge (which correlates with $2 \mathrm{G}$ ), nominal magnetic dipole moment (which also correlates with $2 \mathrm{G}$ ), and rest mass (which correlates with $4 \mathrm{G}$ ). An example of an ongoing modeling anomalous property is anomalous magnetic dipole moment. Detecting effects of $\Sigma G$ for which $\Sigma \geq 10$ might be difficult. (See discussion related to equation (140.) This essay does not necessarily recommend which one of equation (48) and equation (49) pertains to nature. (See discussion regarding equation (134) and equation (135).)

$$
\begin{gathered}
\Sigma_{\max }=8 \\
\Sigma_{\max }=20
\end{gathered}
$$

\subsection{Modeling leading to the table of elementary particles}

We discuss concepts and methods that lead to the table of known and suggested elementary particles. (See table 23.)

We provide perspective regarding processes leading to development of the table. 
Ideally, we might use a method that features notions that we might call small data-sets and smalldata techniques. An input small data-set could be the set of known elementary particles. The small-data techniques could feature not very many formulas or other mathematics techniques. The output would feature a presumably-small data-set of all elementary particles that nature includes.

Some aspects of table 23 point to possibilities for the scenario that we just described. The table exhibits organizing principles. One principle features a choice between values of $\Sigma$. One principle features a choice between non-zero mass and zerolike mass.

Some aspects of table 23 point to possible difficulties regarding the scenario that we just described. The notion of zerolike mass correlates with ongoing modeling KMS modeling. For some models, zerolike means zero. For some models zerolike means - for some elementary particles - non-zero. More generally, ongoing modeling KMS modeling includes models that use the notion of potential energy and, thereby, might bypass some needs to consider elementary bosons. Some of those models correlate with classical physics. Some of those models correlate with quantum physics (and, for example, with the Schrodinger equation).

Similar ambiguities pertained regarding the periodic table for chemical elements. There were two organizing principles - atomic weight and similarity regarding chemical interactions. (Perhaps, note reference [5].) People originally did not understand bases for those principles. Neither principle proved to be strictly rigorous. After people developed nuclear physics modeling and atomic physics modeling, people better understood the principles and the chemical elements.

Our method features an input small data-set that is the set of known elementary particles. The output features a small data-set that might include all elementary particles that nature includes. (See table 23.)

We characterize our method as using (non-computerized or mental) techniques that correlate with the two-word term machine learning and with the two-element term big-data techniques.

A pivotal aspect of the method features the following steps. Recognize that some parts of a partial differential equation, which ongoing modeling uses for KMS PDE modeling, seem to encode information correlating with ongoing modeling KMS modeling for potentials that correlate with electromagnetism and with the strong interaction. Use the equation in a context of proposed modeling PFS PDE modeling. Anticipate that solutions correlating with the equation will correlate with simple particles. This duality - that some particles (which correlate with root forces) correlate with the equation and some particles (which correlate with simple particles) correlate with solutions to the equation - portends some complexity regarding the method.

Another pivotal aspect of the method features the notion that one can use PFS ALG modeling to represent elementary particles and to add (compared to results from PFS PDE modeling) information about conservation-law symmetries that pertain. However, without inputs based on PFS PDE modeling, PFS ALG modeling could point to an overly large set of candidate elementary particles.

The method has iterative aspects. Look at data and modeling. Reuse, extend, create, or integrate modeling. Match, explain, predict, or reinterpret data. Iterate.

Assuming that our modeling proves useful, the possibility that people can gain more understanding becomes relevant.

Regarding the periodic table for chemical elements, gaining new understanding correlated with developing nuclear physics and with developing atomic physics.

If we assume that (at least fermion) elementary particles are truly elementary, gaining more understanding (might include embracing a notion of dark matter isomers but) would not necessarily feature deeper aspects of nature. (Here, this essay uses the two-word phrase truly elementary advisedly, from a standpoint of modeling. This essay de-emphasizes the topic of the extent to which nature might exhibit aspects that people might consider to be more elementary than elementary particles.) New understanding could feature new modeling. Aspects of UMS modeling might point to how to develop a so-called theory of everything. (See discussion related to table 110.) UMS modeling might point not only to all elementary particles and their properties but also to an adequately encompassing set of quantum mechanics modeling regarding motion and classical mechanics modeling regarding motion.

We provide perspective regarding aspects of the table of elementary particles (or, table 23).

Table 24 summarizes some possible correlations between observed phenomena, ongoing modeling, and proposed modeling. For each row in the table, proposed modeling suggests that the item in the third column might explain aspects correlating with the other two columns. (Regarding the entries that allude to one or more components of $4 \mathrm{G}$ forces, see table 62 and specifically see table $62 \mathrm{~b}$. Regarding the notion of isomers, see tables $20,27 \mathrm{~b}, 27 \mathrm{~d}, 62 \mathrm{~b}$, and 63 .)

Table 25 reviews and previews correlations that core proposed modeling includes. (See tables $12 \mathrm{~b}$, 19, 20, and 58.) We provide table 25 to provide context and perspective for our discussing how proposed 
Table 24: Some possible correlations between observed phenomena, ongoing modeling, and proposed modeling (with specifics about $4 \mathrm{G}$ forces correlating with the notion of isomers and with the notion of components)

\begin{tabular}{lll}
\hline Phenomenon & Ongoing modeling & Proposed modeling \\
\hline & Quantum vacuum & Aye and jays \\
& Inflaton & Aye \\
& Inflationary dark energy & Components of 4G forces \\
Accelerating expansion & Dark energy negative pressure & Component of 4G forces \\
Neutrino oscillations & At least one non-zero neutrino rest mass & $8 \mathrm{G}$ forces \\
Some astrophysics data & At least one non-zero neutrino rest mass & 8G forces \\
Nuclear physics & Attractive residual strong force & Pie \\
Nuclear physics & Repulsive residual strong force & Jays \\
\hline
\end{tabular}

Table 25: Some possible correlations between root forces and phenomena (with the three-word term conserved and additive referring to a sum - across objects - of values of the property)

\begin{tabular}{llcc}
\hline$\Sigma \Phi$ & Property of an object & Conserved and additive & XA oscillator pair \\
\hline $2 \mathrm{G}$ & Charge & Yes & 1,2 \\
$4 \mathrm{G}$ & Rest energy & No & 3,4 \\
$6 \mathrm{G}$ & Freeable energy (which, for simple & No & 5,6 \\
& fermions only, correlates with generation) & & \\
$8 \mathrm{G}$ & 3LB number & Yes & 7,8 \\
$2 \mathrm{U}$ & Color charge & No & 9,10 \\
\hline
\end{tabular}

modeling develops table 23 .

For $2 \mathrm{G}, 4 \mathrm{G}$, and $2 \mathrm{U}$, table 25 shows seemingly expected correlations between proposed modeling and ongoing modeling. For example, in each of core proposed modeling and core ongoing modeling, photons (or, regarding proposed modeling, $2 \mathrm{G}$ ) interact with charge and nominal magnetic moment.

Core ongoing modeling (and, as far as we know, also unverified ongoing modeling) does not include aspects that would correlate directly with $6 \mathrm{G}$ or $8 \mathrm{G}$. Core ongoing modeling includes the notion of internal energy above a ground state energy.

\subsubsection{Proposed elementary particle modeling (or, PEPM)}

We continue discussion regarding proposed elementary particle modeling (or, PEPM). (See discussion related to tables 5 and 6 .)

Mathematics and ongoing modeling include partial differential equations pertaining to isotropic harmonic oscillators. A partial differential equation correlating with an isotropic multidimensional quantum harmonic oscillator includes an operator that correlates with $r^{-2}$ and an operator that correlates with $r^{2}$. (See equations (3) and (4).) We consider KMS modeling. (See table 17.) The symbol $r$ denotes a radial spatial coordinate. The $r^{-2}$ operator in equation (4) can model aspects correlating with the square of an electrostatic potential. The potential correlates with $r^{-1}$ and can be either attractive or repulsive. The force correlates with $r^{-2}$ and can be either attractive or repulsive. The $r^{-2}$ operator can model aspects correlating with the square of a gravitational potential. The $r^{-2}$ operator can model aspects correlating with each G-family force $\Sigma G$ for which $\Sigma \leq 8$. (See table 25.) The $r^{2}$ operator in equation (3) can model aspects correlating with the square of a strong interaction potential. Ongoing modeling includes the concept of asymptotic freedom. The potential correlates with $r^{1}$. The force correlates with $r^{0}$. (Apparently, over time, ongoing modeling discussion might have de-emphasized a possible correlation between asymptotic freedom and the notion that aspects of a potential that might approach - at sufficiently large distance $-r^{1}$ behavior pertains.) This strong interaction potential would correlate with excitations related to the $2 \mathrm{U}$ subfamily (or, gluons) and with interactions within hadron-like particles. (Ongoing modeling includes within the two-word term strong force the notion of a residual strong force. The three-word term residual strong force pertains to interactions between hadron-like particles. Proposed modeling suggests correlating an attractive component of the residual strong force with the so-called 0P - or, pie - simple boson. Proposed modeling suggests correlating a repulsive component of the residual strong force with the so-called 2J - or, jay - simple bosons.)

Equations (3) and (4) seem to encode information (about potentials and forces) that correlate with G-family and U-family elementary particles. We pursue the notion that solutions to equations (3) and (4) might correlate with aspects that pertain to some elementary particles. Our use of equations (3) and (4) to match and predict elementary particles correlates with PFS modeling. (Technically, such use 
of equations (3) and (4) to match and predict elementary particles does not necessarily depend on the extent to which either of the electromagnetic interaction or the gravitational interaction correlates with a potential that correlates with $r^{-1}$. Technically, such use of equations (3) and (4) to match and predict elementary particles does not necessarily depend on the extent to which the strong interaction correlates with a potential that correlates with $r^{1}$. We might anticipate that people will develop integrative modeling that better integrates modeling that we use. Perhaps, people will - to develop such integrative modeling - use notions that correlate with information that table 57 shows. Perhaps, people will - to develop such integrative modeling - use notions that people would correlate with UMS modeling and with results from UMS modeling. This essay de-emphasizes developing such integrative modeling.)

Proposed modeling PFS PDE modeling might point to results pertaining to other than the G family and the U family. For example, the next two sentences might pertain. Operator aspects that correlate with $r^{0}$ might correlate with simple fermions. Operator aspects that correlate with $r^{0}$ might correlate with aspects of the weak interaction. (Here, the expression $r^{0}$ does not necessarily correlate with all non-residual aspects of the strong interaction.)

Table 26 outlines steps that our modeling takes. (This table symbolizes steps. Understanding this table is not necessary for understanding aspects below in this essay.) For each step, the leftmost column lists items that correlate with inputs to the step. The next column notes modeling concepts that are key to taking the step. The rightmost column lists items that correlate with outputs from the step. PFS modeling pertains. (See table 17.) In table 26a, the first step uses the notion that correlates aspects of PDE modeling with potentials that we associate with root forces. The steps output a list of elementary particles. In table $26 \mathrm{~b}$ steps output masses. Table $26 \mathrm{c}$ shows possible steps that this essay generally de-emphasizes. Table 26d discusses symbols that appear in tables 26a, 26b, and 26c. Equation (50) explains the notation $\left|\iota_{3 C H}\right| \stackrel{\prime}{=}$. (Each one of tables 26a, 26c, and 26d exhibits use of this notation.)

$$
\left|\iota_{3 C H}\right| \stackrel{=}{=} \text { denotes }\left|\iota_{3 C H}\right|=n \text { or } 0
$$

We discuss objects and properties.

Each of ongoing modeling and proposed modeling includes the notion of an object. (See table 14.) Models for an object may include notions of internal properties upon which all observers would agree. One such property is charge (or, charge that people would observe in the context of a frame of reference in which the object does not move). Models for objects may include notions of kinematics properties upon which observers might legitimately disagree. One such notion is velocity, relative to the observers, of an object. Models can include notions of interactions between objects. An interaction can change for an object - at least one of some internal properties and some kinematics properties.

Table 27 lists some properties that people attribute to objects. PEPM tends to work from table $27 \mathrm{a}$ toward table $27 \mathrm{e}$. In contrast, development of aspects of ongoing modeling, including QFT (or, quantum field theory), has emphasized - from early on in the development of ongoing modeling - aspects correlating with table $27 \mathrm{e}$. The symbol $q_{\epsilon}$ denotes the charge of an electron. The symbol $c$ denotes the speed of light. In table 27a, $S$ correlates with the $S$ in the expression $S(S+1) \hbar^{2}$ and not necessarily with a notion of spin with respect to a particular axis. In tables $27 \mathrm{~b}$ and $27 \mathrm{~d}$, the notion of isomers correlates with the topic of dark matter and with aspects of tables $62 \mathrm{~b}, 63$, and 89 . In table $27 \mathrm{c}$, the use of the symbol $S$ does not correlate with notions of spin. (Compare with, for example, table 27a.) Elsewhere, this essay tends to de-emphasize discussing entropy and does not use the symbol $S$ to pertain to entropy. The symbol NR denotes the two-word phrase not relevant.

Each of the symbols, except $m$, in table 27 a denotes a quantity that is always an integer. Each of the quantities in table 27a pertains for each elementary particle. Each - except generations - of the quantities in table 27 a can pertain for objects that contain more than one elementary particle. In terms of measurements, equation (51) pertains. The symbol $\varepsilon_{0}$ denotes the vacuum permittivity.

$$
\iota_{3 C H}=1 \text { correlates with }\left(\left|q_{\epsilon}\right| / 3\right) /\left(4 \pi \varepsilon_{0}\right)^{1 / 2}
$$

Table 28 lists aspects correlating with some symmetries that table 13 lists. (See table 18. Perhaps, compare with table 19. Perhaps, note table 34. Perhaps, note table 40.)

We discuss the notion of double-entry bookkeeping.

Ongoing modeling includes modeling, for photons, that features mathematics correlating with two harmonic oscillators. Ongoing modeling correlates modeling for each of two polarization modes with one harmonic oscillator. Each mode can correlate with a spatial dimension that is orthogonal to both the direction of motion of the photon and to the spatial dimension correlating with the other mode. These notions correlate with KMS modeling. 
Table 26: Steps, regarding modeling

(a) Steps that output elementary particles

\begin{tabular}{clc}
\hline From & Via & To \\
\hline$\Sigma \mathrm{G}, 2 \mathrm{U}$ & PFS PDE, PFS ALG & $0 \mathrm{H}, 1 \mathrm{C}, 1 \mathrm{~N}, 2 \mathrm{~W}$ \\
$1 \mathrm{C}, 1 \mathrm{~N}, 2 \mathrm{~W}$ & $\left|\iota_{3 C H}\right| \doteq 3 \rightarrow\left|\iota_{3 C H}\right|=(2$ or 1$)$ & $1 \mathrm{Q}, 1 \mathrm{R}, 2 \mathrm{~T}$ \\
$2 \mathrm{~W}$ & $\mathrm{UMS}$ ALG & $2 \mathrm{~J}$ \\
$0 \mathrm{H}$ & $n_{X A 0}=0 \leftarrow n_{X A 0}=-1$ & $0 \mathrm{I}$ \\
$2 \mathrm{U}$ & $S U(3) \rightarrow S U(3) \uplus I$ & $0 \mathrm{P}$ \\
\hline
\end{tabular}

(b) Steps that output boson masses

\begin{tabular}{clc}
\hline From & Via & To \\
\hline$\Sigma \mathrm{G}$ & PFS ALG, PFS PDE & $0 \mathrm{H}, 2 \mathrm{~W}$ \\
$0 \mathrm{H}, 2 \mathrm{~W}$ & $n_{X A 0}=0 \leftarrow n_{X A 0}=-1$ & $0 \mathrm{I}, 2 \mathrm{~J}$ \\
$\Sigma \mathrm{G}$ & PFS ALG, PFS PDE & $2 \mathrm{~T}$ \\
Ongoing & $m_{\pi}$ & $0 \mathrm{P}$ \\
\hline
\end{tabular}

(c) Possible steps that the modeling de-emphasizes

\begin{tabular}{clc}
\hline From & Via & To \\
\hline $2 \mathrm{U}$ & PFS ALG & $(\geqq 4) \mathrm{U}$ \\
$0 \mathrm{H}$ & $\left|\iota_{3 \mathrm{CH}}\right| \stackrel{=}{=} \rightarrow\left|\iota_{3 C H}\right| \dot{=}(2$ or 1$)$ & $\%$ \\
\hline
\end{tabular}

(d) Explanations regarding some symbols

Discussion

- The symbol $\iota$ denotes an integer.

- The symbol $\iota_{3 C H}$ denotes charge, in units of one-third the negative of the charge of an electron (or, in units of the negative of the charge of a down quark).

- $\left|\iota_{3 C H}\right|^{\prime} 3 \rightarrow\left|\iota_{3 C H}\right| \stackrel{\prime}{=}(2$ or 1$)$ denotes extending results for $\left|\iota_{3 C H}\right| \stackrel{\prime}{=} 3$ to results for $\left|\iota_{3 C H}\right| \stackrel{\prime}{=} 2$ and to results for $\left|\iota_{3 C H}\right| \stackrel{\prime}{=}$.

- The expression $n_{X A 0}=0 \leftarrow n_{X A 0}=-1$ denotes - for each of XA equals TA and XA equals SA - substituting the number minus one for the number zero.

- $S U(3) \rightarrow S U(3) \uplus I$ denotes extending modeling to, in effect, include the identity operator, which operator-centric modeling regarding $S U(3)$ lacks.

- The word ongoing denotes aspects of ongoing modeling that model the attractive component of the residual strong force via modeling that includes notions of virtual pions.

- The symbol $m_{\pi}$ denotes the mass (or masses) of pions.

- The notation $\not$ denotes the notion that this essay generally de-emphasizes the concept $\mathrm{X}$. 
Table 27: Some properties of objects

(a) Invariant properties of objects

\begin{tabular}{ccll}
\hline Symbol & Definition & Concept & Related symbol (ongoing modeling) \\
\hline$\iota_{3 C H}$ & & 3CH (charge, in units of $\left.\left|q_{\epsilon}\right| / 3\right)$ & $q$ - charge \\
$m$ & & rest mass, in units of energy $/ c^{2}$ & $m$ - rest mass \\
$j$ & $1 \leq j \leq 3$ & generation, for simple fermions & 1 or 2 or 3 \\
$\iota_{S}$ & $=2 S$ & spin, in units of $\hbar / 2$ & $S$ - spin (nonnegative), in units of $\hbar$ \\
$\iota_{L}$ & $=3 L$ & lepton number, in units of $3 L$ & $L$ - lepton number (integer) \\
$\iota_{B}$ & $=3 B$ & baryon number, in units of $3 B$ & $B$ - baryon number (integer $\times 1 / 3)$ \\
$\iota_{3 L B}$ & $=3(L-B)$ & 3LB number (or, 3LB) & \\
\hline
\end{tabular}

(b) Other invariant property of elementary particles (proposed modeling)

\begin{tabular}{|c|c|c|c|}
\hline Symbol & Definition & Concept & $\begin{array}{l}\text { Related symbol } \\
\text { (ongoing } \\
\text { modeling) }\end{array}$ \\
\hline- & relevant isomers & $\begin{array}{l}\text { a list of isomers of charge (or, a list of } \\
\text { isomers of simple particles) with which an } \\
\text { excitation of the elementary particle } \\
\text { correlates }\end{array}$ & - \\
\hline
\end{tabular}

(c) Other properties

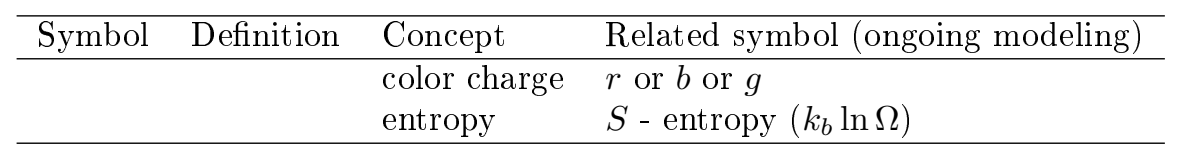

(d) Other invariant property of the universe (proposed modeling)

\begin{tabular}{|c|c|c|c|}
\hline Symbol & Definition & Concept & $\begin{array}{l}\text { Related symbol } \\
\text { (ongoing } \\
\text { modeling) }\end{array}$ \\
\hline$\iota_{I}$ & $=\mathrm{NR}, 1,6$, or 36 & $\begin{array}{l}\text { number of isomers of charge (or, number of } \\
\text { isomers of simple particles) }\end{array}$ & - \\
\hline
\end{tabular}

(e) Observer-centric properties

\begin{tabular}{cll}
\hline Symbol & Concept & Related symbol (ongoing modeling) \\
\hline$E$ & energy, in units of energy & $E$ \\
$\vec{P}$ & momentum, in units of momentum & $\vec{P}$ \\
$\vec{J}$ & angular momentum, in units of angular momentum & $\vec{J}$ \\
\hline
\end{tabular}


Table 28: Aspects - of modeling - that correlate with some TA-side aspects and some SA-side aspects

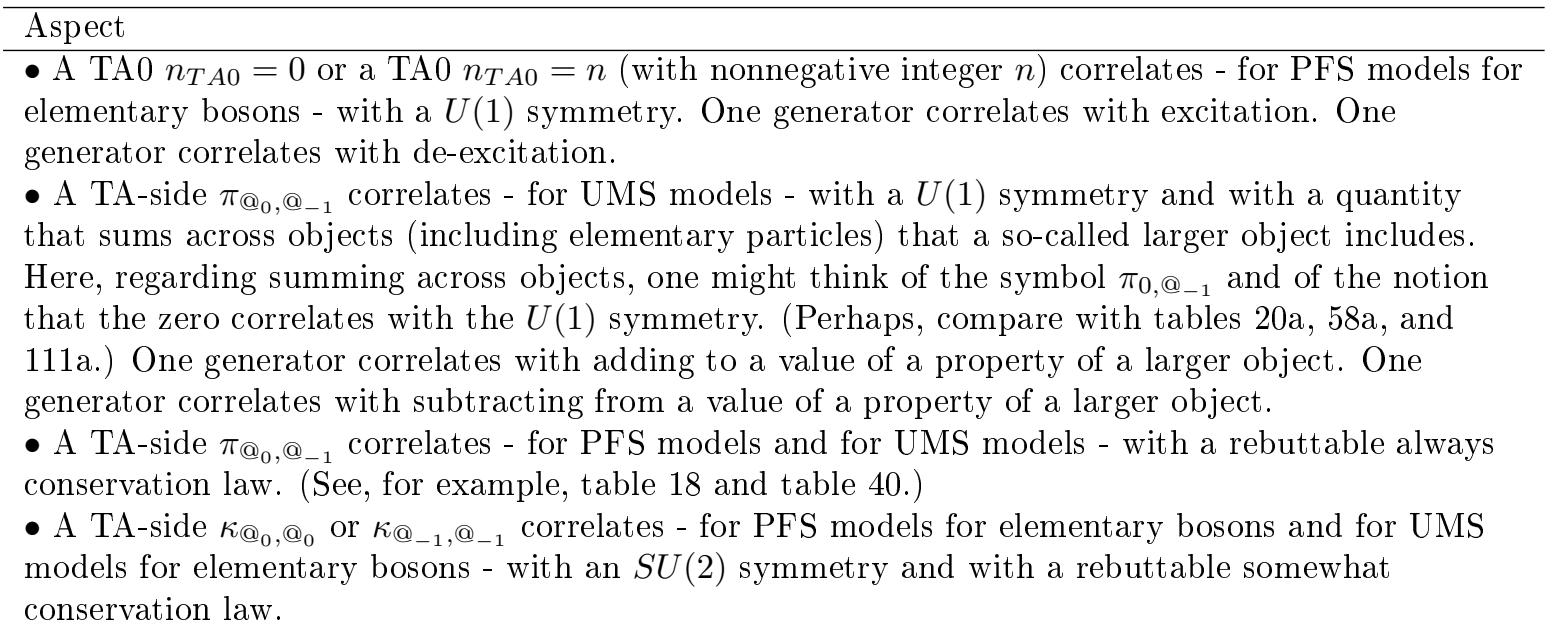

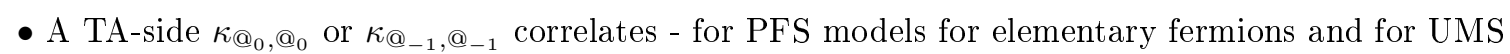
models for elementary fermions - with (for TA5-and-TA6) an $S U(2)$ symmetry and with a rebuttable always conservation law.

- A TA-side $\kappa_{@_{0}} @_{-1}$ correlates - for PFS models for elementary bosons and for UMS models for elementary bosons - no symmetry and with a so-called not necessarily conservation law. (See table 18 and table 40.)

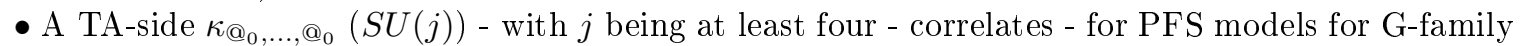
elementary bosons - with a not necessarily conservation law regarding fermion generation. (See, for example, table 48b. Technically the notation correlates with $j$ - not two - TA-side oscillators.)

- A TA-side $\kappa_{@_{-1}, \ldots, @_{-1}}(S U(3))$ - correlates - for PFS models for U-family elementary bosons with a rebuttable always conservation law regarding fermion generation. (See discussion regarding table 54. Technically the notation correlates with three - not two - TA-side oscillators.)

- An SA-side $\pi_{n, @_{0}}$ or $\pi_{0, @_{0}}$ correlates - for PFS models for G-family elementary bosons - with two modes. One mode correlates with left circular polarization. One mode correlates with right circular polarization.

- An SA-side $\pi_{@_{0}, @_{-1}}$ correlates - for PFS models for elementary fermions and for UMS models for elementary fermions - with (for SA1-and-SA2) particle and antiparticle and with (for SA7-and-SA8) positive 3LB number and negative 3LB number.

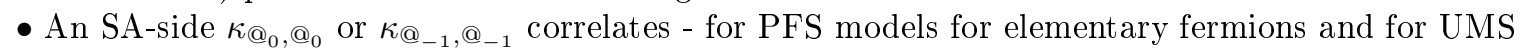
models for elementary fermions - with an $S U(2)$ symmetry and with (for SA5-and-SA6) three generations or (for SA9-and-SA10) three color charges.

- An SA-side $\kappa_{@_{0}, @_{-1}}$ correlates - for PFS models for elementary bosons - with no dependence (regarding interaction strengths) regarding the property that correlates with the relevant oscillator pair. (For example, the Higgs boson does not interact with charge. See table 47, )

- An SA-side $\pi_{@_{0}, @_{0}}$ correlates - for PFS models for G-family elementary bosons - with no dependence (regarding interaction strengths) regarding the property that correlates with the relevant oscillator pair. (For example, the graviton - or 4G - does not interact with charge. See table 48a.)

- An SA-side $\kappa_{@_{0}} @_{0}, \kappa_{0,0}, \kappa_{@_{-1}, @_{-1}}$, or $\kappa_{-1,-1}$ might correlate - for UMS models - with an $S U(2)$ symmetry and with a trio of degrees of freedom for aspects that sum across objects. For example, regarding charge, the three degrees of freedom correlate with negative charge, zero charge, and positive charge. Regarding 3LB, the three degrees of freedom correlate with negative 3LB, zero 3LB, and positive 3LB. Regarding momentum, ongoing modeling correlates with three spatial dimensions. Also, regarding the jay (or, 2J) bosons, the three degrees of freedom correlate with three simple elementary particles. (For the jay bosons, the notion of summable property correlates with 3LB. See table 52 .) 
Proposed modeling PFS ALG modeling has bases in the concept that modeling photons based on four harmonic oscillators has uses. The concept has bases in the ongoing modeling notion of KMS modeling based on four dimensions. One of those four dimensions is temporal. The other three of those four dimensions are spatial. The concept points to equation (38) and to a concept to which we apply the two-element term double-entry bookkeeping. The term refers to ALG modeling that maintains a numeric balance between TA-side aspects and SA-side aspects. The balance reflects a notion that a sum pertaining to TA-side aspects equals a sum pertaining to SA-side aspects.

Proposed modeling PDE modeling also exhibits aspects that we correlate with the two-element term double-entry bookkeeping. Here, the balance refers to effects of a TA-side quantum operator and to effects of an SA-side quantum operator. (See, for example, equation (31).)

\subsubsection{Patterns regarding properties of known elementary particles}

We discuss possibilities regarding an analog - to the periodic table for chemical elements - for elementary particles.

The periodic table reflects properties of chemical elements. (Note reference [5].) One relevant property is the types of chemical interactions in which an element participates. One relevant property is the atomic weight. A usual display of the periodic table features an array with columns and rows. Elements listed in a column participate in similar interactions. For a row, the atomic weight of an element is usually greater than the atomic weight for each element to the left of the subject element. Atomic weights in one row exceed atomic weights in rows above the subject row.

We look for patterns regarding the known elementary particles. (See table 23.)

Table 29 reflects a concept that - for particles for which $m>0$ pertains - the number of elementary particles in a subfamily correlates with the spin of the elementary particles in the subfamily. Table $29 \mathrm{~b}$ explains notation that table 29 a uses. The spin $S$ correlates with an overall angular momentum for which the expression $S(S+1) \hbar^{2}$ pertains. The spin $S$ does not depend on a choice of an axis. Each of the three columns that correlate with the one-element label sub-hadronic correlates with a magnitude of charge that differs from the magnitude of charge pertaining to the other two columns labeled sub-hadronic.

Equation 52 pertains for known elementary particles for which $m>0$. (See table 29c.) Spin and the number of particles relate to each other.

$$
\iota_{S}+1=\sum n
$$

\subsubsection{Some applications of PDE mathematics}

Table 30 notes some applications of modeling that people can base on the mathematics that underlies PDE modeling. (See table 17.) Applications for which the table shows the symbol ${ }^{\dagger}$ pertain regarding supplementary proposed modeling. Applications for which the table shows the symbol ${ }^{\dagger}$ are generally not directly necessary for core proposed modeling work regarding elementary particles, astrophysics, and cosmology. We assume that ongoing modeling kinematics and dynamics modeling generally suffices. Each of ongoing modeling and proposed modeling can use KMS applications.

Table 17 discusses some aspects regarding PDE modeling. (Perhaps, note work leading to equation (31).) For KMS modeling, the variable t can correlate with ongoing modeling notions of temporal aspects and the variable $\mathrm{r}$ can correlate with ongoing modeling notions of spatial aspects. Solutions $\Psi$ can correlate with wave functions. For PFS modeling, the variable t does not necessarily correlate with ongoing modeling notions of temporal aspects and the variable $r$ does not necessarily correlate with ongoing modeling notions of spatial aspects. Solutions $\Psi$ do not necessarily correlate with the ongoing modeling notion of wave functions.

\subsubsection{PDE aspects of proposed elementary particle modeling}

We discuss modeling correlating with the first row in table 30. The notion of PFS modeling pertains. The expression $\nu_{S A}<0$ pertains.

This work features the numbers of dimensions that equations (53) and (54) show. Even though our work here features PFS modeling, people might want to consider the extent to which equation (53) correlates with a KMS modeling notion of three spatial dimensions. A possible SA-side aspect features correlations between numbers of simple particles, which would be a PFS modeling topic, and numbers of spin states, which would be a KMS modeling topic. (See discussion related to table 29 and equation $(52)$.)

$$
D_{S A}^{*}=3
$$


Table 29: Known elementary particles

(a) Elementary particles

\begin{tabular}{|c|c|c|c|c|c|}
\hline \multirow[t]{2}{*}{$\iota_{S}=2 S$} & Can be free & Can be free & Sub-hadronic & Sub-hadronic & Sub-hadronic \\
\hline & $m>0$ & $m \doteq 0$ & $m>0$ & $m>0$ & $m \doteq 0$ \\
\hline 0 & $0 \mathrm{H}^{0}: 1$ & & & & \\
\hline 1 & $1 C_{j}^{3}: 2$ & $1 \mathrm{~N}_{j}^{0}:\{1$ or 2$\}$ & $1 \mathrm{Q}_{j}^{2}: 2$ & $1 \mathrm{Q}_{j}^{1}: 2$ & \\
\hline 2 & $2 W^{0}: 1$ & & & & \\
\hline 2 & $2 W^{3}: 2$ & & & & \\
\hline 2 & & $2 \mathrm{G}^{0}:((2))$ & & & $2 \mathrm{U}^{0}: 8$ \\
\hline 4 & & $\left\{4 \mathrm{G}^{0}:((2))\right\}$ & & & \\
\hline
\end{tabular}

(b) Notation

\begin{tabular}{ccl}
\hline Notation & Note & Discussion \\
\hline$\iota_{S}$ & & Spin, in units of $\hbar / 2$ \\
& & Spin, in units of $\hbar$ \\
& Can be free & Can move independently of hadrons (Perhaps, see table 37$)$ \\
Sub-hadronic & Found only (in today's universe) in hadrons \\
$m \doteq 0$ & Non-zero mass & The mass is at least the mass of an electron \\
$\iota_{S} \Phi^{\left|\iota_{3 C H}\right|}$ & Zerolike mass & Models (in some models) as having zero mass \\
& $\iota_{S} \Phi$ & A subfamily of the $\Phi$ family of elementary particles \\
& $\left|\iota_{3 C H}\right|$ & Charge in units of one-third the charge of a positron \\
& $|x|$ & The absolute value of $x$ \\
& $\overline{N R}$ & Generation, for fermions, with $1 \leq j \leq 3 ;$ NR for bosons \\
$: n$ & $n=1$ or 2 & Not relevant \\
& $\{1$ or 2$\}$ & Majorana fermion or Dirac fermion, respectively \\
& 8 & Number of gluons \\
$:((2))$ & & One particle with two modes \\
$\left\{\iota_{S} \Phi_{-}^{\iota_{3 C H}}\right\}$ & & Hypothetical subfamily (hypothesized, but not yet found) \\
& $\left\{4 \mathrm{G}^{0}:((2))\right\}$ & Graviton (hypothesized, but not yet found) \\
\hline
\end{tabular}

(c) Subfamilies for which $m>0$ (with the one-element term gen denoting the word generation)

\begin{tabular}{cccc}
\hline Subfamily and (if not NR) gen & Particles & $\sum n($ per $: n)$ & $\Sigma+1$ \\
\hline $0 \mathrm{H}$ & Higgs boson & 1 & 1 \\
$1 \mathrm{C}_{j}$ & $j$-th generation of charged leptons & 2 & 2 \\
$1 \mathrm{Q}_{j}^{2}$ & $j$-th generation of $\left|\iota_{3 C H}\right|=2$ quarks & 2 & 2 \\
$1 \mathrm{Q}_{j}^{1}$ & $j$-th generation of $\left|\iota_{3 C H}\right|=1$ quarks & 2 & 2 \\
$2 \mathrm{~W}$ & $\mathrm{Z}$ and $\mathrm{W}\left(\mathrm{W}^{-3}\right.$ and $\left.\mathrm{W}^{+3}\right)$ bosons & 3 & 3 \\
\hline
\end{tabular}

(d) Subfamilies for which $m=0$ (with the one-element term gen denoting the word generation)

\begin{tabular}{ccc}
\hline Subfamily and (if not NR) gen & Particles & $\sum n$ (per $\left.: n\right)$ \\
\hline $1 \mathrm{~N}_{j}$ & $j$-th generation of neutrinos & $\{1$ or 2$\}$ \\
$2 \mathrm{G}$ & Photon & $((2))$ \\
$\{4 \mathrm{G}\}$ & Graviton (hypothetical) & $((2))$ \\
$2 \mathrm{U}$ & Gluons & 8 \\
\hline
\end{tabular}

(e) A note regarding neutrinos

\begin{tabular}{l}
\hline Note \\
- Tables 29a, 29b, and $29 \mathrm{~d}$ comport with ongoing modeling notions that neutrinos might model \\
either as Majorana fermions or as Dirac fermions. Proposed modeling suggests that lepton number \\
minus baryon number is a conserved quantity. As a result, proposed modeling suggests that \\
neutrinos model as Dirac fermions and not as Majorana fermions. (See, for example, equation \\
$(179$.$) Regarding each one of tables 29 \mathrm{a}, 29 \mathrm{~b}$, and $29 \mathrm{~d}$, proposed modeling suggests that the \\
replacement $\{1$ or 2$\} \leftarrow 2$ is appropriate.
\end{tabular}


Table 30: Some applications of modeling that people can base on the mathematics that underlies PDE modeling (with the symbol ${ }^{\dagger}$ denoting applications that pertain regarding supplementary proposed modeling and generally are not directly necessary for core proposed modeling work regarding elementary particles, astrophysics, and cosmology)

\begin{tabular}{|c|c|c|c|c|}
\hline Application & ... modeling & PFS or KMS & $\nu_{S A}$ & Focus \\
\hline $\begin{array}{l}\text { Simple particles that } \\
\text { nature embraces }\end{array}$ & Proposed & PFS & $<0$ & One simple particle \\
\hline $\begin{array}{l}\text { Interaction vertices that } \\
\text { modeling includes }{ }^{\dagger}\end{array}$ & Proposed & PFS & $<0$ & Multiple elementary particles \\
\hline $\begin{array}{l}\text { Modeling for some } \\
\text { aspects of excitations }\end{array}$ & $\begin{array}{l}\text { Ongoing or } \\
\text { proposed }\end{array}$ & KMS & $\geq 0$ & Multi-object system \\
\hline $\begin{array}{l}\text { Modeling for some } \\
\text { aspects of dynamics }\end{array}$ & $\begin{array}{l}\text { Ongoing or } \\
\text { proposed }\end{array}$ & KMS & $\geq 0$ & Multi-object system \\
\hline
\end{tabular}

Table 31: PDE notions that pertain for some PFS modeling applications

Notion

- The symbol $S$ denotes spin divided by $\hbar$. The symbol $\hbar$ denotes the reduced Planck's constant.

- For some solutions - which comport with equation $(55)$ - to equation (8), $D_{S A} \neq D_{S A}^{*}$.

- Solutions for which $\nu_{S A}=-1 / 2$ can correlate with notions of fields for simple fermions.

- Solutions for which $\nu_{S A}=-1$ can correlate with notions of fields for simple bosons.

- Solutions for which $\nu_{S A}=-3 / 2$ can correlate with notions of particles for simple fermions.

- TA-side PDE solutions are radial with respect to $t$, the TA-side analog to the SA-side radial coordinate $r$.

- For some TA-side PDE solutions, $D_{T A} \neq D_{T A}^{*}$.

$$
D_{T A}^{*}=1
$$

We anticipate making PFS modeling uses of equations (55) and (56). Here, each of $2 S$ and $2 S_{T A}$ is a nonnegative integer. (We de-emphasize using the symbol $S_{S A}$ instead of the symbol $S$.) The case that features equation (55), $\sigma_{S A}=+1$, and $S=\nu_{S A}$ is a restating of equation (8). (Ongoing modeling KMS modeling features expressions of the form that equation (57) shows.) The case that features equation (55) and $\sigma_{S A}=-1$ correlates with some aspects of proposed modeling models. (See discussion related to table 76.) Similar concepts pertain regarding equation (56) and $\sigma_{T A}$.

$$
\begin{gathered}
\Omega_{S A}=\sigma_{S A} S\left(S+D_{S A}^{*}-2\right)=\sigma_{S A} S(S+1), \text { for } \sigma_{S A}= \pm 1 \\
\Omega_{T A}=\sigma_{T A} S_{T A}\left(S_{T A}+D_{T A}^{*}-2\right)=\sigma_{T A} S_{T A}\left(S_{T A}-1\right), \text { for } \sigma_{T A}= \pm 1
\end{gathered}
$$

$$
\hbar^{2} S(S+1) \text {, for nonnegative integer } 2 S
$$

Table 31 lists notions that pertain for some PFS modeling applications.

Along with mathematics correlating with three dimensions and $D_{S A}^{*}=3$ and with mathematics correlating with one dimension and $D_{T A}^{*}=1$, we anticipate needing mathematics correlating with two dimensions and a case that we denote by $D^{\prime \prime}=2$. (For example, discussion above does not adequately cover the topic of notions of particles for simple bosons. The case of $D^{\prime \prime}=2$ is relevant to - at least notions of particles for simple bosons.)

Table 32 shows some relationships between some PDE parameters. The symbol XA can denote either $\mathrm{SA}$ or TA. Here, we correlate with $D^{\prime \prime}$ the symbols $S^{\prime \prime}, \nu^{\prime \prime}, \Omega^{\prime \prime}$, and $\sigma^{\prime \prime}$. Each of $S^{\prime \prime}, \nu^{\prime \prime}, \Omega^{\prime \prime}$, and $\sigma^{\prime \prime}$ does not necessarily correlate with uses of $S, \nu_{S A}, \Omega_{S A}, \sigma_{S A}, S_{T A}, \nu_{T A}, \Omega_{T A}$, or $\sigma_{T A}$ in models regarding simple particles. For $\Omega^{\prime \prime}=0$, the table uses the letters NR to denote that the sign of $\sigma^{\prime \prime}$ is not relevant. For table $32 \mathrm{~b}$ we use equation (25) to develop the relevant expressions for $D$ and to calculate values of $D$. Similar methodology pertains regarding $D$ in tables $32 \mathrm{c}, 32 \mathrm{~d}$, and $32 \mathrm{e}$. (When considering tables $32 \mathrm{~b}$, $32 \mathrm{c}, 32 \mathrm{~d}$, and $32 \mathrm{e}$, perhaps note that calculations of $D$ do not involve values of $D_{S A}^{*}, D_{T A}^{*}$, and $D^{\prime \prime}$.)

\subsubsection{PDE modeling regarding simple particles}

We explore bounds regarding the simple particles that proposed modeling suggests. 
Table 32: Relationships between some PDE parameters

(a) Relationships relevant to $D_{X A}^{*}$ and $D^{\prime \prime}$ (with XA denoting either SA or TA)

\begin{tabular}{|c|c|c|c|c|c|}
\hline$D_{X A}^{*}$ & $D^{\prime \prime}$ & $\nu_{X A}$ & $\nu^{\prime \prime}$ & $D_{X A}^{*}+2 \nu_{X A}$ & $D^{\prime \prime}+2 \nu^{\prime \prime}$ \\
\hline 1 & & $-1 / 2$ & & 0 & \\
\hline 1 & & -1 & & -1 & \\
\hline 1 & & $-3 / 2$ & & -2 & \\
\hline & 2 & & -1 & & 0 \\
\hline 3 & & $-1 / 2$ & & 2 & \\
\hline 3 & & -1 & & 1 & \\
\hline 3 & & $-3 / 2$ & & 0 & \\
\hline
\end{tabular}

(b) SA-side relationships, for $\sigma_{S A}=+1$ (with $D$ denoting $D_{S A}$; with $\Omega \operatorname{denoting} \Omega_{S A}$; and with * denoting a possible cause for concern regarding a possible lack of normalization)

\begin{tabular}{cccccccccc}
\hline$\nu_{S A}$ & $D$ & $S$ & $\Omega_{S A}$ & $\sigma_{S A}$ & $D$ & $D+2 \nu_{S A}$ & $D_{S A}^{*}+2 \nu_{S A}$ & Re simple particles & $\iota_{S}$ \\
\hline-1 & $3-\Omega$ & 0 & 0 & +1 & 3 & 1 & 1 & Boson field & 0 \\
$-1 / 2$ & $(5-4 \Omega) / 2$ & $1 / 2$ & $3 / 4$ & +1 & 1 & 0 & 2 & Fermion field & 1 \\
$-3 / 2$ & $(21-4 \Omega) / 6$ & $1 / 2$ & $3 / 4$ & +1 & 3 & 0 & 0 & Fermion particle & 1 \\
-1 & $3-\Omega$ & 1 & 2 & +1 & 1 & $-1^{*}$ & 1 & Boson field & 2 \\
\hline
\end{tabular}

(c) TA-side relationships, for $\sigma_{T A}=+1$ (with $D$ denoting $D_{T A}$; with $\Omega$ denoting $\Omega_{T A}$; and with * denoting a possible cause for concern regarding a possible lack of normalization)

\begin{tabular}{cccccccccc}
\hline$\nu_{T A}$ & $D$ & $S_{T A}$ & $\Omega_{T A}$ & $\sigma_{T A}$ & $D$ & $D+2 \nu_{T A}$ & $3+2 \nu_{T A}$ & Re simple particles & $\iota_{S}$ \\
\hline-1 & $3-\Omega$ & 0 & 0 & +1 & 3 & 1 & 1 & Boson field & 0 \\
$-1 / 2$ & $(5-4 \Omega) / 2$ & $3 / 2$ & $3 / 4$ & +1 & 1 & 0 & 2 & Fermion field & 1 \\
$-3 / 2$ & $(21-4 \Omega) / 6$ & $3 / 2$ & $3 / 4$ & +1 & 3 & 0 & 0 & Fermion particle & 1 \\
-1 & $3-\Omega$ & 1 & 2 & +1 & 1 & $-1^{*}$ & 1 & Boson field & 2 \\
\hline
\end{tabular}

(d) SA-side relationships, for $\sigma_{S A}=-1$ (with $D$ denoting $D_{S A}$; and with $\Omega$ denoting $\left.\Omega_{S A}\right)$

\begin{tabular}{cccccccc}
\hline$\nu_{S A}$ & $D$ & $S$ & $\Omega_{S A}$ & $\sigma_{S A}$ & $D$ & $D+2 \nu_{S A}$ & $2 S+1$ \\
\hline$-1 / 2$ & $(5-4 \Omega) / 2$ & $1 / 2$ & $-3 / 4$ & -1 & 4 & 3 & 2 \\
$-1 / 2$ & $(5-4 \Omega) / 2$ & $3 / 2$ & $-15 / 4$ & -1 & 10 & $\ldots$ & $\ldots$ \\
$-1 / 2$ & $(5-4 \Omega) / 2$ & $\ldots$ & $\ldots$ & $\ldots$ & $\ldots$ & $\ldots$ & $\ldots$ \\
-1 & $3-\Omega$ & 0 & 0 & -1 & 3 & 1 & 1 \\
-1 & $3-\Omega$ & 1 & -2 & -1 & 5 & 3 & 3 \\
-1 & $3-\Omega$ & 2 & -6 & -1 & 9 & $\ldots$ & $\ldots$ \\
-1 & $3-\Omega$ & $\ldots$ & $\ldots$ & $\ldots$ & $\ldots$ & $\ldots$ & $\ldots$ \\
$-3 / 2$ & $(21-4 \Omega) / 6$ & $1 / 2$ & $-3 / 4$ & -1 & 4 & 1 & 2 \\
$-3 / 2$ & $(21-4 \Omega) / 6$ & $3 / 2$ & $-15 / 4$ & -1 & 6 & $\ldots$ & $\ldots$ \\
$-3 / 2$ & $(21-4 \Omega) / 6$ & $\ldots$ & $\ldots$ & $\ldots$ & $\ldots$ & $\ldots$ & $\ldots$ \\
\hline
\end{tabular}

(e) Relationships between some parameters, for $D^{\prime \prime}=2$ and $D^{\prime \prime}+2 \nu^{\prime \prime}=$ 0 (with NR denoting that the sign of $\sigma^{\prime \prime}$ is not relevant)

\begin{tabular}{cccccccc}
\hline$\nu^{\prime \prime}$ & $D$ & $S^{\prime \prime}$ & $\Omega^{\prime \prime}$ & $\sigma^{\prime \prime}$ & $D$ & $D+2 \nu^{\prime \prime}$ & $2 S^{\prime \prime}+1$ \\
\hline-1 & $3-\Omega^{\prime \prime}$ & 1 & 1 & +1 & 2 & 0 & 3 \\
-1 & $3-\Omega^{\prime \prime}$ & 0 & 0 & $\mathrm{NR}$ & 3 & 1 & 1 \\
-1 & $3-\Omega^{\prime \prime}$ & 1 & -1 & -1 & 4 & 2 & 3 \\
-1 & $3-\Omega^{\prime \prime}$ & 2 & -4 & -1 & 7 & 5 & 5 \\
-1 & $3-\Omega^{\prime \prime}$ & 3 & -9 & -1 & 12 & 10 & 7 \\
-1 & $3-\Omega^{\prime \prime}$ & 4 & -16 & -1 & 19 & 17 & 9 \\
-1 & $3-\Omega^{\prime \prime}$ & 5 & -25 & -1 & 28 & 26 & 11 \\
-1 & $3-\Omega^{\prime \prime}$ & 6 & -36 & -1 & 39 & 37 & 13 \\
-1 & $3-\Omega^{\prime \prime}$ & 7 & -49 & -1 & 52 & 50 & 15 \\
-1 & $3-\Omega^{\prime \prime}$ & 8 & -64 & -1 & 67 & 65 & 17 \\
-1 & $3-\Omega^{\prime \prime}$ & 9 & -81 & -1 & 84 & 82 & 19 \\
\hline
\end{tabular}


Table 33: Aspects that PEPM posits to correlate with modeling for simple elementary particles

\begin{tabular}{l}
\hline Aspect \\
- Each simple elementary fermion correlates with a subfamily for which a $\nu_{S A}=-1 / 2$ solution \\
exists. The solution correlates with the notion of fields for the elementary particles in the \\
subfamily. The solution correlates with the notion of volume-like. \\
- Each simple elementary fermion correlates with a subfamily for which a $\nu_{S A}=-3 / 2$ solution \\
exists. The solution correlates with the notion of particles for the elementary particles in the \\
subfamily. The solution correlates with the notion of point-like. \\
- Each simple elementary boson correlates with a subfamily for which a $\nu_{S A}=-1$ solution exists. \\
The solution correlates with the notion of fields for the elementary particles in the subfamily. The \\
solution correlates with the notion of volume-like. \\
- Each simple elementary boson correlates with a subfamily for which a $\nu_{S A}=-1$ solution exists \\
for each of three oscillator pairs. The trio of solutions correlates with the notion of particles for the \\
elementary particles in the subfamily. The solutions correlate with the notion of point-like. \\
- For each such solution, the relevant $\Omega \ldots$ is nonnegative, the relevant $\sigma \ldots$ is plus one, the relevant \\
$2 S \ldots$ is a nonnegative integer, and the relevant $D$ is a positive integer. \\
\hline
\end{tabular}

Table 33 lists aspects that PEPM posits to correlate with modeling for simple elementary particles. (See table 31.) The table limits the range of relevant subfamilies. The table does not specify the number of subfamilies that nature embraces.

The order of rows in table $32 \mathrm{~b}$ correlates with non-decreasing values of $\Omega_{S A}$. A value of spin $S$ correlates with the value of $\Omega_{S A}$. Proposed modeling posits that each simple elementary particle correlates with a field. Proposed modeling posits that $D$ must be a positive integer. (Also, note that, for this discussion, $D=D_{S A}$.) No larger values of $S$ comport with equation (58). (For example, for fermion fields, $S=3 / 2$ would correlate with $\Omega_{S A}=15 / 4$ and with a negative value, -5 , for $D_{S A}$.) Equation (59) correlates with a limit that pertains regarding simple particles. (See table 33. Also, our assumptions regarding the existence of simple particles include excluding solutions for which $\sigma_{S A}=-1$. See table $32 \mathrm{~d}$. If we included solutions for which $\sigma_{S A}=-1$, table $32 \mathrm{~d}$ indicates a possibility for indefinitely large values of $S$.) We do not expect that nature embraces simple particles with spins other than zero, one-half, and one.

$$
\begin{gathered}
S \geq 0 \text { and } D \geq 1 \\
0 \leq S \leq 1
\end{gathered}
$$

We explore modeling regarding the simple particles that proposed modeling suggests. This exploration pertains within the bounds that equations (58) and (59) imply.

Tables $32 \mathrm{~b}$ and $32 \mathrm{c}$ show solutions that correlate with fields for all relevant cases. Tables $32 \mathrm{~b}$ and $32 \mathrm{c}$ show solutions that correlate with particles for all relevant elementary fermion cases. The tables do not discuss particles for relevant elementary boson cases.

Table $32 \mathrm{~b}$ includes a column with label $D_{S A}^{*}+2 \nu_{S A}$. We use the symbol $D^{\prime}$ to denote $D_{S A}^{*}$. Table $32 \mathrm{c}$ includes a column with label $3+2 \nu_{T A}$. We use the symbol $D^{\prime}$ to denote the three. These two columns comport with the notion that a relevant $D^{\prime}+2 \nu_{X A}$ should be positive for fields and zero for particles. For each of tables $32 \mathrm{~b}$ and $32 \mathrm{c}, D^{\prime}=3$.

We pursue discussion based on relevance of the three TA-side oscillators TA0, TA1, and TA2 and three SA-side oscillators SA0, SA1, and SA2. (Compare with equation (37).)

In general, use of equation (37) allows separation of terms into clusters. Equation (37) is a sum of $D_{X A}$ terms. Each one of the $D_{X A}$ terms appears in exactly one cluster. For $D_{X A}=1$, there is one term (which correlates with the XA0 oscillator) and one cluster (which contains the one term). For $D_{X A}=3$, we use two clusters. One cluster correlates with the XA0 oscillator. One cluster correlates with the XA1-and-XA2 oscillator pair. In these and similar cases, we apply - for each two-oscillator cluster - an analog to equations (3) and (4).

Here, specifically, $D_{T A}=D_{S A}=D^{\prime}=3$.

We anticipate aspects regarding modeling - for fields and particles - for simple bosons and simple fermions.

For each of fields for simple bosons and fields for simple fermions, modeling points to the notion that, for relevant choices of sets of oscillators and of $D$, equation 60 pertains. For fields for simple bosons, 
$\nu_{S A}=-1$. For fields for simple fermions, $\nu_{S A}=-1 / 2$. The notion of volume-like correlates with equation $(60)$.

$$
D+2 \nu_{S A}=1
$$

For each of particles for simple bosons and particles for simple fermions, modeling points to the notion that, for relevant choices of sets of oscillators and of $D$, equation (61) pertains. For particles for simple bosons, $\nu_{S A}=-1$. For particles for simple fermions, $\nu_{S A}=-3 / 2$. The notion of point-like correlates with equation (61).

$$
D+2 \nu_{S A}=0
$$

We anticipate that, for some purposes, the substitutions that equations (62) and (63) show are useful. (The notation $\mathrm{a} \leftarrow \mathrm{b}$ denotes the notion that $\mathrm{b}$ replaces - or substitutes for - a.)

$$
\begin{aligned}
& \mathrm{TA} 1 \leftarrow \mathrm{TA} 5, \mathrm{TA} 2 \leftarrow \mathrm{TA} 6 \\
& \mathrm{SA} 1 \leftarrow \mathrm{SA} 5, \mathrm{SA} 2 \leftarrow \mathrm{SA} 6
\end{aligned}
$$

We discuss modeling for fields for simple bosons.

Regarding modeling for fields for $\iota_{S}=2$ simple bosons, one can use the notion of mapping the $D=1$ solutions - that tables $32 \mathrm{~b}$ and $32 \mathrm{c}$ show - into the three dimensions that correlate with $D^{\prime}=3$. (For each of PFS modeling and KMS modeling, the $D=1$ solution has or would have no dependence on angular coordinates.) Here, each one of the SA-side solution and the TA-side solution normalizes and correlates with equation (60). $\left(D_{S A}^{*}+2 \nu_{S A}\right.$ is one. $3+2 \nu_{T A}$ is one.) We correlate the three oscillators $\mathrm{SA0}$, $\mathrm{SA} 1$, and SA2 with the SA-side solution. The following sentences pertain regarding the weak interaction (or, Z and W) bosons. (Similar remarks pertain regarding tweak bosons.) SA-side use of $D^{\prime}=3$ is compatible with (PFS modeling and) the existence of three elementary boson states (the $\mathrm{Z}$ boson, the negatively charged $\mathrm{W}$ boson, and the positively charged $\mathrm{W}$ boson). (See table 42, SA-side use of $D^{\prime}=3$ is compatible with (KMS modeling and) the existence of three spin states. Also, SA-side use of $D^{\prime}=3$ is compatible with (UMS modeling and) the existence of three elementary boson states (the Z boson, the negatively charged $\mathrm{W}$ boson, and the positively charged $\mathrm{W}$ boson). (See the XA1-XA2 row in table 58.) TA-side use of $D^{\prime}=3$ is compatible with (PFS modeling and) rebuttable somewhat conservation of fermion generation. (See table 34 and discussion related to table 42.) We invoke equation (62). The oscillator pair TA5-and-TA6, correlates with and, in effect, contains the $\kappa_{@_{0}, @_{0}}($ or, $S U(2))$ symmetry that is relevant to rebuttable somewhat conservation of fermion generation.

Regarding modeling for fields for $\iota_{S}=0$ simple bosons, one can use results that tables $32 \mathrm{~b}$ and $32 \mathrm{c}$ show. For each of XA equals $\mathrm{SA}$ and XA equals TA, $D^{\prime}=3$ and $D^{\prime}+2 \nu_{X A}=1$. For each of XA equals SA and XA equals TA, equation (60) pertains. We invoke each of equations (62) and (63). (See, for example, table 47.) Rebuttable somewhat conservation of fermion generation pertains.

We discuss modeling for particles for simple bosons.

For simple bosons, we expect that modeling regarding particles correlates with the equations $D^{\prime \prime}=2$, $\nu^{\prime \prime}=-1$ and $D+2 \nu^{\prime \prime}=0$. (See tables 33 and 32e.) We base this expectation on the notion that, for simple fermions, modeling regarding particles correlates with the expression $D_{T A}+2 \nu_{T A}=0=D_{S A}+2 \nu_{S A}$. (See equation (61) and tables $32 \mathrm{~b}$ and $32 \mathrm{c}$.)

Regarding modeling for particles for simple bosons, we start from the $D_{T A}=D_{S A}=D^{\prime}=3$ models for fields. We use the clusters TA1-and-TA2 (or, after applying equation (62), TA5-and-TA6), TA0-andSA0, and SA1-and-SA2. For each cluster, we use the equations $D^{\prime \prime}=2, \nu^{\prime \prime}=-1$ and $D+2 \nu^{\prime \prime}=0$.

Regarding modeling for particles for $\iota_{S}=2$ simple bosons, notions - such as three oscillator pairs and rebuttable somewhat conservation of fermion generation - that pertain for fields for $\iota_{S}=2$ simple bosons continue to pertain. Paralleling work regarding fields for $\iota_{S}=2$ simple bosons, we invoke equation (62).

Regarding modeling for particles for $\iota_{S}=0$ simple bosons, the two perhaps seemingly extra oscillator pairs - TA1-and-TA2 and SA1-and-SA2 - correlate with the notion of rebuttable somewhat conservation of fermion generation. Paralleling work regarding fields for $\iota_{S}=0$ simple bosons, we invoke equations (62) and (63).

We discuss modeling for fields for simple fermions.

Regarding modeling for fields for $\iota_{S}=1$ simple fermions, the $D_{S A}^{*}+2 \nu_{S A}$ column in table $32 \mathrm{~b}$ shows a value of two. The $3+2 \nu_{T A}$ column in table $32 \mathrm{c}$ shows a value of two. Seemingly, equation (60) might not pertain. 
Table 34: Some symmetries and interpretations that pertain to all elementary fermions

\begin{tabular}{|c|c|c|}
\hline Pair & Symmetry & Interpretation \\
\hline SA1-and-SA2 & $\pi_{@_{0}, @_{-1}}$ & This symmetry correlates with matter and antimatter. \\
\hline SA5-and-SA6 & $S U(2)$ & $\begin{array}{l}\text { The three generations of elementary fermions correlate with the three } \\
\text { generators of } S U(2) \text {. For ALG modeling, each of the symbols } \kappa_{@_{0}, @_{0}} \\
\text { and } \kappa_{@_{-1}, @_{-1}} \text { can correlate with the } S U(2) \text { symmetry. }\end{array}$ \\
\hline
\end{tabular}

We focus on aspects that correlate with fields that correlate with fermion subfamilies $1 \Phi$.

Regarding fields for elementary fermions, modeling can feature an effective $D_{\dagger}=2$ instead of $D^{\prime}=3$. (Each elementary fermion correlates with one - not two - values for handedness. For example, each known matter elementary fermion correlates with left handedness and not with right handedness. Each known antimatter elementary fermion correlates with right handedness and not with left handedness. A reduction from $D^{\prime}=3$ dimensions to $D_{\dagger}=2$ correlates, in effect, with the lack - for each particle - of a second handedness. Perhaps, note discussion - regarding photon modes - related to table 35. Also, perhaps, note table 10.) For $D_{\dagger}=2, D_{\dagger}+2 \nu_{S A}=D_{\dagger}+2 \nu_{T A}=1$. The notions of volume-like and field still pertain. Equation (60) pertains.

We focus on aspects that correlate with fields that correlate with individual elementary particles (or, individual generations) within fermion subfamilies $1 \Phi$.

We shift our attention to aspects that are somewhat separate from aspects correlating with $D_{\dagger}=2$. From $D_{1}=D^{\prime}=3$, proposed modeling applies the transformation that correlates with equation (19). (Perhaps note that, in equation (19), $j=2$ and that, regarding discussion here, $j \nu_{S A}$ is an integer.) The result $D_{2}=(2 \cdot 3)-2=4$ pertains. We bring together aspects correlating with $D_{\dagger}=2$ and aspects correlating with $D_{2}=4$. The result $D_{2}-D_{\dagger}=4-2=2$ pertains. In effect, the transformation - from $D_{1}$ to $D_{2}$ adds - compared to models for which $D_{\dagger}=2$ pertains - two TA-side oscillators and two SA-side oscillators. Each new oscillator pair can correlate with an $S U(2)$ symmetry. (See table 13.) Equation (60) pertains for each of $D=D_{\dagger}=2$ and $D=D_{2}-D_{\dagger}=2$. Elsewhere, we correlate (for modeling for elementary fermion fields and for modeling for elementary fermion particles, the additional two TA-side oscillators with the TA5-and-TA6 pair and the two additional SA-side oscillators with the SA5-and-SA6 pair. (See, for example table 58.) We invoke equations (62) and (63). The SA5-and-SA6 pair correlates with - for each elementary fermion - three generations.

Table 34 shows and interprets symmetries that pertain to all elementary fermions. (Note tables 28 and 58.) For each elementary fermion, either $@_{0}$ correlates with three of the four relevant oscillators or $@_{-1}$ correlates with three of the four relevant oscillators.

We discuss modeling for particles for $\iota_{S}=1$ simple fermions.

Table $32 \mathrm{~b}$ shows $D=3$ and $D+2 \nu_{S A}=0$. Table $32 \mathrm{c}$ shows $D=3$ and $D+2 \nu_{T A}=0$. Equation (61) pertains. The number $D=3$ correlates - regarding table 34 - with one of the number of SA-side $@_{0}$ and the number of SA-side $@_{-1}$. Results that table 34 features pertain. One can reuse results that pertain for fields for $\iota_{S}=1$ simple fermions.

\subsubsection{Concepts regarding representations for photons}

We discuss notions that, with respect to ongoing modeling, correlate with KMS modeling.

Ongoing modeling describes photon states via two harmonic oscillators. Ongoing modeling features four space-time-coordinate dimensions.

Why not describe photon states via four harmonic oscillators?

Proposed modeling describes photon states via ALG modeling that features four harmonic oscillators.

The four-oscillator models correlate with PFS modeling.

One might assume that four-oscillator models must correlate with non-zero longitudinal polarization and with a photon rest mass that would be non-zero. However, mathematics allows a way to avoid this perceived possible problem. (See equation (35).)

One might assume that using four oscillators would add no insight. However, using four oscillators leads to a framework for expressing aspects of proposed modeling and leads to insight about a family of phenomena that includes photons.

\subsubsection{ALG representations for elementary particles}

We discuss aspects of ALG modeling.

We consider the left circular polarization mode of a photon. We denote the number of excitations of the mode by $n$. Here, $n$ is a nonnegative integer. One temporal oscillator pertains. We label that oscillator 
Table 35: A PFS-centric representation for excitations for the left circular polarization mode of a photon

\begin{tabular}{cccccccccc}
\hline Side & 0 & 1,2 & 3,4 & 5,6 & 7,8 & 9,10 & 11,12 & 13,14 & 15,16 \\
\hline TA & $n$ & & & & & & & & \\
SA & -1 & $n, @_{0}$ & & & & & & & \\
\hline
\end{tabular}

Table 36: A PFS-centric representation for excitations for a photon

\begin{tabular}{cccccccccc}
\hline Side & 0 & 1,2 & 3,4 & 5,6 & 7,8 & 9,10 & 11,12 & 13,14 & 15,16 \\
\hline TA & $n$ & $\pi_{@_{0}, @_{-1}}$ & & & & & & & \\
SA & -1 & $\pi_{n, @_{0}}$ & & & & & & & \\
\hline
\end{tabular}

TA0. The excitation number $n_{T A 0}=n$ pertains. Here, $n_{T A 0}=n \geq 0$ pertains. Harmonic oscillator mathematics correlates a value of $n+1 / 2$ with that oscillator. Three spatial oscillators pertain. Here, $n_{S A 0}=-1, n_{S A 1}=n, n_{S A 2}=@_{0}$. Oscillator SA0 correlates with longitudinal polarization and has zero amplitude for excitation. (See equation (35).) Oscillator SA1 correlates with left circular polarization. Oscillator SA2 correlates with right circular polarization. The symbol @ denotes a value of _that, within a context, never changes. For left circular polarization, $@_{0}$ pertains for oscillator SA2. The sum $n+1 / 2$ correlates with each of the one TA-side oscillator and the three SA-side oscillators. For the TAside oscillator, the sum - with which we correlate the symbol $A_{T A}^{A L G}$ - equals $(n+1 / 2)$. For the SA-side oscillators, the sum - with which we correlate the symbol $A_{S A}^{A L G}$ - equals $(-1+1 / 2)+(n+1 / 2)+(0+1 / 2)$.

Table 35 shows excitations for the left circular polarization mode of a photon. (See table 12b. Perhaps, compare with table 20a.) The two-element term PFS-centric representation correlates with the notion that a more complete PFS representation exists. In the case of table 35, a more complete PFS representation would show information correlating with, for example, conservation of charge and conservation of 3LB. (See, for example, table 41)

For the right circular polarization mode of a photon, one exchanges the values of $n_{S A 1}$ and $n_{S A 2}$. The result is $n_{S A 1}=@_{0}, n_{S A 2}=n$.

Table 36 shows excitations for a photon. Photons interact with charge. We assume that the oscillator pair SA1-and-SA2 correlates with charge or with interactions with charge. Photons do not change the charges of objects with which photons interact. Per table 28, the TA1-and-TA2 entry in table 35 correlates with rebuttable always conservation of charge. Per table 13 , this term makes a zero contribution to $A_{T A}^{A L G}$. Proposed modeling does not correlate the TA1-and-TA2 entry in table 35 with excitation of photons or with de-excitation of photons.

For each mode, for the TA0 oscillator, raising operators and lowering operators that correlate with $U(1)$ symmetry pertain. (Perhaps, note table 28.) One generator correlates with excitation. One generator correlates with de-excitation. This essay does not fully explore the extent to which this $U(1)$ symmetry correlates with the $U(1)$ symmetry that the elementary particle Standard Model associates with photons. (Perhaps, note discussion regarding equation (173).)

The representation that table 36 shows is invariant with respect to observer. In interpreting a measurement, each observer would correlate the measurement with the same one of left circular polarization and right circular polarization. For that polarization, each observer, in effect, would measure the same value of $n$. Observers might disagree with respect to measured values of energy or momentum.

We prepare to explore representations for elementary particles other than photons.

Table 37 discusses our uses - regarding elementary particles, regarding objects in general, and regarding environments in which elementary particles and other objects exist - of the word unfree and of the word free.

Table 38 discusses aspects regarding modeling for elementary bosons for which $n_{T A 0}=-1$. Absent such aspects, models might correlate with the notion that such bosons would not excite. (Note equation $(35)$.)

Table 39 lists questions that, for this immediate discussion, we de-emphasize fully addressing.

Table 40 discusses aspects regarding elementary boson TA-side symmetries. (See table 18. Perhaps, see table 28.) We interpret core ongoing modeling as correlating with the proposed modeling TA7-andTA8 notion of always - when summed over interacting objects - conservation of 3LB number. (Core ongoing modeling does not include aspects correlating with the proposed modeling notion of the $2 \mathrm{~T}$ subfamily. Reference [6] discusses observational evidence that we interpret as supporting the proposed modeling TA7-and-TA8 notion of always conservation of 3LB number.) Elsewhere, we discuss aspects that correlate with the explicit mentions, in table 40, of $2 \mathrm{~W}$ (See table 42.), 4G (See table 48a.), 6G (See 
Table 37: The word unfree and the word free - regarding elementary particles, objects in general, and environments

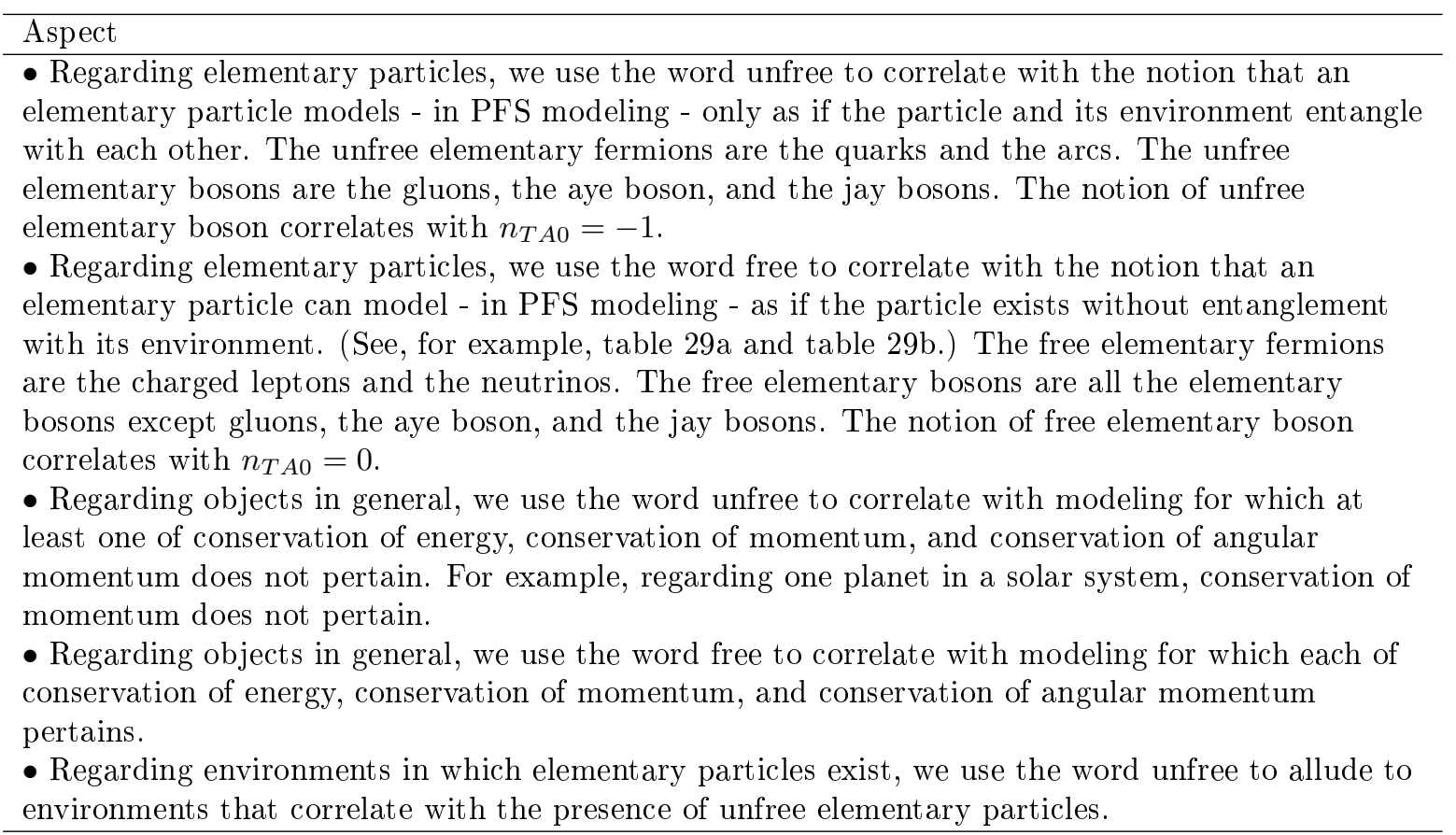

Table 38: Aspects regarding modeling elementary bosons for which $n_{T A 0}=-1$

Aspect

- Elementary bosons that correlate with $n_{T A 0}=-1$ can excite based on - to use a word from ongoing modeling - entanglement with their environments.

- Modeling for excitations can correlate with a notion of $n_{T A 0}=(-1)^{+}$. (See, for example, discussion related to table 68 and equation (81).)

Table 39: Questions regarding interpreting the equation $n_{S A 0}=-1$

Question
- To what extent do answers to the following questions differ between simple bosons and simple
fermions?
- To what extent does $n_{S A 0}=-1$ correlate with zero longitudinal polarization?
- To what extent does $n_{S A 0}=-1$ correlate with zero rest mass?
- To what extent does $n_{S A 0}=-1$ correlate with being able to excite a state via using an
arbitrarily small amount of energy squared?
- To what extent does $n_{S A 0}=-1$ correlate, for free environments, with travel at the speed of light?
- To what extent does $n_{S A 0}=-1$ correlate with inabilities to interact with phenomena, such as
the Higgs boson, that proposed modeling associates with the SA0 oscillator?


Table 40: Aspects regarding elementary boson TA-side symmetries (with the assumption that each elementary fermion correlates - for each relevant TA-side oscillator pair - with the notion of exact symmetry)

\begin{tabular}{|c|c|c|c|c|}
\hline \multicolumn{5}{|l|}{ Aspect } \\
\hline \multicolumn{5}{|c|}{ - The following correlations pertain regarding the TA1-and-TA2 oscillator pair. } \\
\hline \multicolumn{2}{|c|}{ Symbol Symmetry } & Conservation & $\begin{array}{l}\text { If this symmetry is } \\
\text { the least exact symmetry, } \\
\text { elementary fermions ... }\end{array}$ & $\begin{array}{l}\text { Elementary } \\
\text { boson } \\
\text { subfamilies }\end{array}$ \\
\hline$\pi_{@_{0}, @_{-1}}$ & Exact & Rebuttable always & do not change charge. & All but ... \\
\hline & Approximate & Rebuttable somewhat & (not relevant) & \\
\hline$\kappa @_{0}, @_{-1}$ & No & Not necessarily & can change charge. & $2 \mathrm{~W}, 2 \mathrm{~T}$ \\
\hline \multicolumn{5}{|c|}{ - The following correlations pertain regarding the TA5-and-TA6 oscillator pair. } \\
\hline $\begin{array}{l}\text { Symbol } \\
\text { (group) }\end{array}$ & \multicolumn{2}{|c|}{ Symmetry $\quad$ Conservation } & $\begin{array}{l}\text { If this symmetry is } \\
\text { the least exact symmetry, } \\
\text { elementary fermions ... }\end{array}$ & $\begin{array}{l}\text { Elementary } \\
\text { boson } \\
\text { subfamilies }\end{array}$ \\
\hline $\begin{array}{l}\kappa_{@_{-1}, \ldots, @_{-}} \\
(S U(3))\end{array}$ & Exact & $\begin{array}{l}\text { (Rebuttable) always } \\
\text { (See table } 113 a \text { ) }\end{array}$ & do not change generations & $2 \mathrm{U}$ \\
\hline $\begin{array}{l}\pi_{@_{0}, @_{-1}} \\
(-)\end{array}$ & Exact & Rebuttable always & do not change generations. & All but ... \\
\hline $\begin{array}{l}\kappa_{\Theta_{0}, @_{0}} \\
(S U(2))\end{array}$ & Approximate & Rebuttable somewhat & might change generations. & $2 \mathrm{~W}, 2 \mathrm{~T}, 4 \mathrm{G}$ \\
\hline $\begin{array}{l}\kappa_{@_{0}, \ldots, @_{0}} \\
(S U(4))\end{array}$ & In effect, no & Not necessarily & can change generations. & $6 \mathrm{G}$ \\
\hline $\begin{array}{l}\kappa_{@_{0}, \ldots, @_{0}} \\
(S U(6)) \\
\end{array}$ & In effect, nc & Not necessarily & can change generations. & $8 \mathrm{G}$ \\
\hline \multicolumn{5}{|c|}{ - The following correlations pertain regarding the TA7-and-TA8 oscillator pair. } \\
\hline Symbol & Symmetry & Conservation & $\begin{array}{l}\text { If this symmetry is } \\
\text { the least exact symmetry, } \\
\text { elementary fermions ... }\end{array}$ & $\begin{array}{l}\text { Elementary } \\
\text { boson } \\
\text { subfamilies }\end{array}$ \\
\hline$\pi_{@_{0}, @_{-1}}$ & Exact & Rebuttable always & do not change 3LB numbers. & All but ... \\
\hline - & Approximate & Rebuttable somewhat & (not relevant) & - \\
\hline$\kappa @_{0}, @_{-1}$ & No & Not necessarily & can change 3LB numbers. & $2 \mathrm{~T}$ \\
\hline
\end{tabular}

table 48b.), 8G (See table 48c), and 2T (See table 52.). Regarding 6G, the three-word phrase in effect no correlates with notions of two - not one - oscillator pairs. Regarding $8 \mathrm{G}$, the three-word phrase in effect no correlates with notions of three - not one - oscillator pairs. Extrapolating backwards, regarding $2 \mathrm{G}$ and the TA5-and-TA6 oscillator pair, an exact symmetry pertains. (See table 41.) We assume that each elementary fermion correlates - for each relevant TA-side oscillator pair - with the notions of exact symmetry and rebuttable always conservation of property. (Note discussion in table 18 about modeling for interactions and about the roles of conservation of property.)

For PFS-centric representations for elementary particles, equation (64) pertains - with the proviso that, for representations for elementary fermions, one does not count the two oscillators that correlate with an $S U(2)$ symmetry that correlates with three generations. Here, $N_{S A}$ denotes a number of relevant SA-side oscillators. (Perhaps, compare equation (64) with equation (52). Also, note that, for each elementary fermion, only one of the two oscillators SA1 and SA2 is relevant. See, for example, discussion - related to table 34 - regarding $D_{\dagger}=2$ and $D^{\prime}=3$.)

$$
N_{S A}=2 S+1
$$

We return to discussing photons.

Table 41 updates table 36 to take into account aspects that table 40 mentions. Reference [7] discusses observational evidence that we interpret as supporting the TA5-and-TA6 notion that correlates with rebuttable always conservation of fermion generation regarding the $2 \mathrm{G}$ subfamily. The TA7-and-TA8 entry correlates with rebuttable always conservation of $3 \mathrm{LB}$ number.

We turn our attention to elementary particles other than photons. (We anticipate roughly following steps that table 26a shows.)

We discuss modeling pertaining to the weak interaction (or, $\mathrm{Z}$ and $\mathrm{W}$ ) bosons.

We note that ongoing modeling interrelates photons and weak interaction bosons. 
Table 41: A PFS-centric representation for excitations for a photon

\begin{tabular}{cccccccccc}
\hline Side & 0 & 1,2 & 3,4 & 5,6 & 7,8 & 9,10 & 11,12 & 13,14 & 15,16 \\
\hline TA & $n$ & $\pi_{@_{0}, @_{-1}}$ & & $\pi_{@_{0}, @_{-1}}$ & $\pi_{@_{0}, @_{-1}}$ & & & & \\
SA & -1 & $\pi_{n, @_{0}}$ & & & & & & & \\
\hline
\end{tabular}

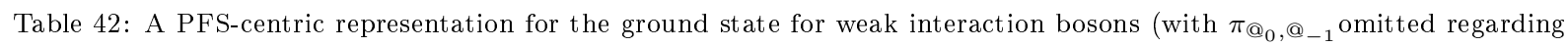
TA7-and-TA8)

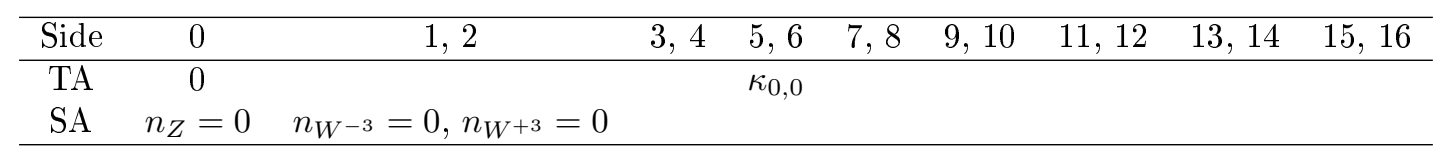

Table 42 posits a ground state for weak interaction bosons. The relevant bosons are the $\mathrm{Z}$ and $\mathrm{W}$ bosons. The table correlates the negative charge state of the $\mathrm{W}$ boson with the SA1 oscillator. The table correlates the positive charge state of the W boson with the SA2 oscillator. (One might correlate negative charge with SA2 and positive charge with SA1. We do not explore this possibility further. This essay does not explore the possibility of a link between such an assignment regarding charge and the assignment of photon circular polarization modes. This essay does not explore the handedness of $\mathrm{W}$ bosons. Perhaps, see reference [8.) Interactions with $\mathrm{W}$ bosons change the charges of elementary fermions. The TA1-andTA2 entry does not show a symmetry that correlates with conservation of charge. (Perhaps, note the equivalence - per table 13 - of [blank] and $\kappa_{0,-1}$.) Placement of $\kappa_{0,0}$ with the TA5-and-TA6 oscillator pair comports with equation (62) and table 20a.

We discuss W-family excitations and we discuss the notion of rebuttable somewhat conservation of fermion generation.

To describe $n$ excitations of the same state of one of the $\mathrm{W}$-family bosons, we use $n_{T A 0}=n=n_{S A}$, with $\mathrm{SA}_{-}$correlating with the one boson. An isolated interaction that excites or de-excites the boson conserves the generation of the fermion that participates in the interaction. For example, an interaction between an electron (or, generation-one charged lepton) and a $\mathrm{W}^{+3}$ boson produces a generation-one neutrino. (Per notation that this essay uses, the charge that correlates with the symbol $\mathrm{W}^{+3}$ equals the charge of a positron. See table 26d.) We say that conservation of generation pertains. We consider some interactions in hadrons (such as protons and neutrons). Here, we consider an entangled emission and absorption of a pair of (perhaps, in the sense of some aspects of ongoing modeling, virtual) W bosons, with one $\mathrm{W}$ boson being a $\mathrm{W}^{-3}$ and the other $\mathrm{W}$ boson being a $\mathrm{W}^{+3}$. Ongoing modeling results suggest that conservation of fermion generation need not pertain for the relevant quarks. Regarding proposed modeling, a transition from the state that table 42 shows to the state characterized by $n_{T A 0}=2, n_{S A 0}=0$, $n_{S A 1}=1$, and $n_{S A 2}=1$ would violate equation (40). The TA-side raising operations would produce a factor of $(1+0)^{1 / 2}(1+1)^{1 / 2}$, which equals $2^{1 / 2}$. The SA-side raising operations would produce a factor of $(1+0)^{1 / 2}(1+0)^{1 / 2}$, which equals 1 . Equations (40) and (41) imply that one of oscillators TA5 and TA6 participates. There are three generations of quarks. Three is the number of generators of $S U(2)$. (See table 13.) We posit that an approximate $S U(2)$ symmetry pertains. We use the five-word term rebuttable somewhat conservation of generation (or, the six-word term rebuttable somewhat conservation of fermion generation). Ongoing modeling seems to correlate this proposed modeling notion of non-conservation of generation with the ongoing modeling notion of CP violation. (See, for example, reference [9].) Proposed modeling suggests the possibility that people might be able to detect non-conservation, induced by $\mathrm{W}$ family effects, of lepton generation. (Reference [9] suggests that people may be on the verge of observing evidence of lepton $\mathrm{CP}$ violation.)

Combining the TA5-and-TA6 $S U(2)$ symmetry with the TA0 $U(1)$ symmetry yields an $S U(2) \times U(1)$ symmetry. (See tables 13 and 42.) This essay does not fully explore possible correlations between this proposed modeling $S U(2) \times U(1)$ symmetry and the $S U(2) \times U(1)$ symmetry that ongoing modeling correlates with the weak interaction. (Perhaps, note discussion regarding equation (173).)

Table 43 shows a UMS-centric representation for the weak interaction bosons. The SA1-and-SA2 $\kappa_{@_{0}, @_{0}}$ correlates with 3 generators, three spin states, and with three charge states. The TA5-and-TA6 $\kappa_{@_{0}, @_{0}}$ correlates with rebuttable somewhat conservation of fermion generation. (See table 18 and table 40.)

We discuss representations for charged leptons and for neutrinos.

For elementary fermions, we posit that ALG modeling does not necessarily need to correlate with excitations. 
Table 43: A UMS-centric representation for the weak interaction bosons (with $\pi_{@_{0}} @_{-1}$ omitted regarding TA7-and-TA8)

\begin{tabular}{cccccccccc}
\hline Side & 0 & 1,2 & 3,4 & 5,6 & 7,8 & 9,10 & 11,12 & 13,14 & 15,16 \\
\hline TA & $@_{0}$ & $\kappa_{@_{0}, @_{-1}}$ & & $\kappa_{@_{0}, @_{0}}$ & & & & & \\
SA & $@_{0}$ & $\kappa @_{0}, @_{0}$ & & & & & & & \\
\hline
\end{tabular}

Table 44: A PFS-centric representation for the subfamily of charged leptons (with $\pi_{@_{0}, @_{-1}}$ omitted regarding TA7-and-TA8)

\begin{tabular}{cccccccccc}
\hline Side & 0 & 1,2 & 3,4 & 5,6 & 7,8 & 9,10 & 11,12 & 13,14 & 15,16 \\
\hline TA & $@_{0}$ & $\pi_{@_{0}, @_{-1}}$ & & & & & & & \\
SA & $@_{0}$ & $\pi_{@_{0}, @_{-1}}$ & & & & & & & \\
\hline
\end{tabular}

Table 44 shows a representation for the $1 \mathrm{C}$ subfamily of charged leptons. The representation comports with the notions of $D^{\prime}=3$ and $D_{\dagger}=2$. (See discussion related to tables $32 \mathrm{~b}, 32 \mathrm{c}$, and 34.) For each of SA1-and-SA2 and TA1-and-TA2, effectively only one oscillator pertains. For SA1-and-SA2, the symbol $\pi_{@_{0}, @_{-1}}$ correlates with the notion of matter particles and antimatter particles. For TA1-and-TA2, the symbol $\pi_{0}, @_{-1}$ correlates with a symmetry that correlates with rebuttable always conservation of charge. For SA0, the symbol $@_{0}$ correlates with non-zero mass and non-zero charge. For TA0, the symbol $@_{0}$ pertains based on double-entry bookkeeping.

Table 45 shows a representation for individual charged leptons. The representation comports with the notions of $D_{1}=D^{\prime}=3$ and $D_{2}=4$. (See discussion related to tables $32 \mathrm{~b}, 32 \mathrm{c}$, and 34.) For SA5-andSA6, the notation $\kappa_{@_{0}} @_{0}$ correlates with the notion of three generations. Per table 28, the TA5-and-TA6 information that table 45 shows correlates with a notion of rebuttable always conservation of fermion generation. (Note interpretations that table 40 describes.)

Table 46 shows a representation for individual neutrinos. Some differences between table 46 and table 45 correlate with two applications of equations (42) and (43). (These applications correlate with interactions that transform a fermion from one of $m>0$ and $m \doteq 0$ to the other of $m>0$ and $m \doteq 0$. Regarding notation, see discussion related to table 23 . Here, the difference between 0 and $@_{0}$ is not material. Here, the difference between -1 and $@_{-1}$ is not material.) One application correlates with oscillator SA0. One application correlates with oscillator TA0. For SA0, the symbol @ $@_{-1}$ correlates with zerolike mass and zero charge. For TA0, the symbol @ $@_{-1}$ pertains based on double-entry bookkeeping. Other differences

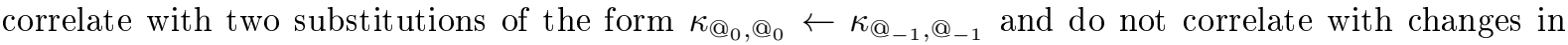
relevant symmetries. The two $\kappa_{@_{0}, @_{0}} \leftarrow \kappa_{@_{-1}, @_{-1}}$ substitutions correlate with the notion that additional oscillators correlate with $n_{X A}=n_{T A 0}$. The notion of $n_{X A}=n_{T A 0}$ is relevant to proposed modeling models regarding refraction. (See discussion related to table $\overline{6} 8$.)

We return to discussing elementary bosons.

Table 47 shows a PFS-centric representation for the ground state for the Higgs boson. Per discussion related to tables $32 \mathrm{~b}$ and $32 \mathrm{c}$, three TA-side oscillators and three SA-side oscillators pertain. The SA-side instance of $\kappa_{@_{0}}, @_{-1}$ correlates with no interactions with charge. Unlike for the weak interaction bosons, no excitements can correlate with a mismatch between $n_{T A 0}$ and a relevant $n_{S A}$. (Note discussion related to table 42.) For Higgs bosons, interactions with elementary fermions correlate with rebuttable always conservation of fermion generation. Hence, table 47 shows $\pi_{@_{0}} @_{-1}$ regarding TA5-and-TA6.

Table 48 shows PFS-centric representations for $4 \mathrm{G}, 6 \mathrm{G}$, and $8 \mathrm{G}$ root forces. Each representation comports with equation (64). Each representation extrapolates from a representation for a photon. (See table 41.) Regarding $4 \mathrm{G}$ and paralleling results regarding the $2 \mathrm{~W}$ subfamily, the TA5-and-TA6 appearance of $\kappa_{@_{0}, @_{0}}$ in table 48 a correlates with rebuttable somewhat conservation of fermion generation. (This leaves the possibility that either modeling that correlates with ongoing modeling notions of virtual interactions or occurrences of multiple close-by interactions could explain neutrino oscillations. However, proposed modeling offers another explanation. See discussion related to equation (142). The proposed modeling treatment seems to explain observed data. Proposed modeling does not necessarily preclude contributions - toward such data - from other phenomena.) Regarding each of table 48b and table 48c,

Table 45: A PFS-centric representation for charged leptons (with $\pi_{@_{0}}$, ${ }_{-1}$ omitted regarding TA7-and-TA8)

\begin{tabular}{cccccccccc}
\hline Side & 0 & 1,2 & 3,4 & 5,6 & 7,8 & 9,10 & 11,12 & 13,14 & 15,16 \\
\hline TA & $@_{0}$ & $\pi_{@_{0}}, @_{-1}$ & & $\kappa_{@_{0}}, @_{0}$ & & & & & \\
SA & $@_{0}$ & $\pi_{@_{0}, @_{-1}}$ & & $\kappa_{@_{0}, @_{0}}$ & & & & & \\
\hline
\end{tabular}




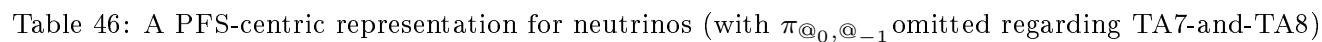

\begin{tabular}{|c|c|c|c|c|c|c|c|c|c|}
\hline Side & 0 & 1,2 & 3,4 & 5,6 & 7,8 & 9,10 & 11,12 & 13,14 & 15,16 \\
\hline TA & $@_{-1}$ & $\pi_{@_{0}, @_{-1}}$ & & $\kappa_{@_{-1}, @_{-1}}$ & & & & & \\
\hline SA & $@_{-1}$ & $\pi_{@_{0}, @_{-1}}$ & & $\kappa_{@_{-1}, @_{-1}}$ & & & & & \\
\hline
\end{tabular}

Table 47: A PFS-centric representation for the ground state for the Higgs boson (with $\pi_{@_{0}}$ @ $_{-1}$ omitted regarding TA1-andTA2 and regarding TA7-and-TA8)

\begin{tabular}{|c|c|c|c|c|c|c|c|c|c|}
\hline Side & 0 & 1,2 & 3,4 & 5,6 & 7,8 & 9,10 & 11,12 & 13,14 & 15,16 \\
\hline TA & 0 & & & $\pi @_{0}, @_{-1}$ & & & & & \\
\hline SA & 0 & $\kappa @_{0}, @_{-1}$ & & & & & & & \\
\hline
\end{tabular}

we use the TA5-and-TA6 item to point to a symmetry that technically correlates with more than just two oscillators.

Table 48 points to the possibility that $\Sigma_{\max }=8$ pertains (Perhaps, see equation (48).) We consider the notion that $n_{T A 0}=0$ correlates with $n_{T A 0}=@_{0}$. We consider the notion that - regarding $4 \mathrm{G}$, $6 \mathrm{G}$, and $8 \mathrm{G}$ respectively - the respective symmetries $S U(3), S U(5)$, and $S U(7)$ pertain. We consider the notion that a limit of $S U(7)$ might pertain. (See, for example, discussion related to equation (46) and discussions related to tables $62 \mathrm{a}$ and 64 . Perhaps, also consider that $20 \mathrm{G}$ does not correlate with $S U(17)$.)

We turn our attention to elementary particles that correlate with $\left|\iota_{3 C H}\right|=2$ or $\left|\iota_{3 C H}\right| \dot{=} 1$. (See table 26d and equation (50).)

Table 49 notes concepts regarding values, for objects, of charge, of $\iota_{3 L B}$, of $L$, and of $B$. Here, we consider that a proton or other hadron with no more than three quarks can correlate with the notion of free. The following notion also pertains. For a hadron-like particle that includes no more than three quarks and arcs, the restrictions to integer charge and integer baryon number preclude the presence of both quarks and arcs.

Proposed modeling suggests a symmetry regarding $\iota_{3 C H}$. The symmetry suggests that each of the cases $\iota_{3 C H}=2$ and $\iota_{3 C H}=1$ is similar to the case $\iota_{3 C H}=3$. For the $1 \mathrm{C}$ subfamily, the cases $\iota_{3 C H}=2$ and $\iota_{3 C H}=1$ correlate with the six $1 \mathrm{Q}$ (or, quark) elementary fermions. Quarks have fractional charges.

Table 50 shows, simple particles that proposed modeling suggests based on the symmetry related to $\iota_{3 C H}$. For the zero-charge $1 \mathrm{R}$ and $2 \mathrm{~T}$ particles that table 50 shows, the number of tick marks in a symbol $\Sigma \Phi^{0}$ - equals $\left|\iota_{3 C H}\right|$.

A representation for each $1 \mathrm{Q}$ particle to which table 50 alludes equals the representation for the corresponding $1 \mathrm{C}$ particle. Table 45 provides a PFS-centric representation for quarks.

A representation for each $1 \mathrm{R}$ particle to which table 50 alludes equals the representation for the corresponding $1 \mathrm{~N}$ particle. Table 46 provides a PFS-centric representation for arcs.

Table 51 provides a UMS-centric representation for tweak bosons. The representation amends the representation that table 43 shows for the weak interaction bosons so as to include not necessarily conservation of fermion 3LB number. (See table 40.)

We discuss the jay bosons.

Table 52 shows a UMS-centric representation for the jay (or, 2J) bosons. The table shows results of applying equations (42) and (43) to a representation for the weak interaction bosons. (See table 43.) The result $n_{S A 0}=-1$ correlates with zero charge and zerolike mass. Paralleling results pertaining to the 2W subfamily, the SA7-and-SA8 $S U(2)$ symmetry correlates with three elementary particles and with three spin states. The SA7-and-SA8 and TA5-and-TA6 items comport with notation-centric conventions regarding $n_{X A}=n_{T A 0}$.

We use the following notation and posit the following notions regarding the three $2 \mathrm{~J}$ bosons. $2 \mathrm{~J}_{0}$ can exhibit left circular polarization and right circular polarization. 2.J_ can exhibit (say) left circular polarization and cannot exhibit right circular polarization. 2 $\mathrm{J}_{+}$can exhibit right circular polarization and cannot exhibit left circular polarization. (Elsewhere, we more fully discuss aspects of jay physics. See table 70 )

We discuss the aye boson.

Table 53 shows a PFS-centric representation for the ground state for the aye boson. Table 53 differs from table 47 - which pertains for the Higgs boson - based on two substitutions - one for TA0 and one for $\mathrm{SA} 0$ - of the form $n_{X A 0}=0 \leftarrow n_{X A 0}=-1$.

We discuss gluons.

Table 54 shows a UMS-centric representation for $2 \mathrm{U}$ forces. (Regarding the use of the oscillator pair 
Table 48: PFS-centric representations for ground states for the 4G, 6G, and 8G root forces

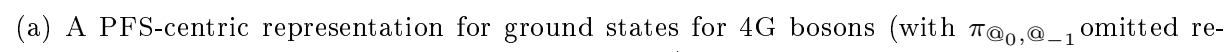
garding TA1-and-TA2 and regarding TA7-and-TA8)

\begin{tabular}{cccccccccc}
\hline Side & 0 & 1,2 & 3,4 & 5,6 & 7,8 & 9,10 & 11,12 & 13,14 & 15,16 \\
\hline TA & 0 & & & $\kappa_{@_{0}, @_{0}}$ & & & & & \\
SA & -1 & $\pi_{@_{0}, @_{0}}$ & $\pi_{0, @_{0}}$ & & & & & & \\
\hline
\end{tabular}

(b) A PFS-centric representation for ground states for 6G bosons (with $\pi_{@_{0}}$ @ $_{-1}$ omitted regarding TA1-andTA2 and regarding TA7-and-TA8)

\begin{tabular}{cccccccccc}
\hline Side & 0 & 1,2 & 3,4 & 5,6 & 7,8 & 9,10 & 11,12 & 13,14 & 15,16 \\
\hline TA & 0 & & & $\kappa_{@_{0}, \ldots, @_{0}}(S U(4))$ & & & & & \\
SA & -1 & $\pi_{@_{0}, @_{0}}$ & $\pi_{@_{0}, @_{0}}$ & $\pi_{0, @_{0}}$ & & & & & \\
\hline
\end{tabular}

(c) A PFS-centric representation for ground states for 8G bosons (with $\pi_{@_{0}}$ @ $_{-1}$ omitted regarding TA1-and-TA2 and regarding TA7-and-TA8)

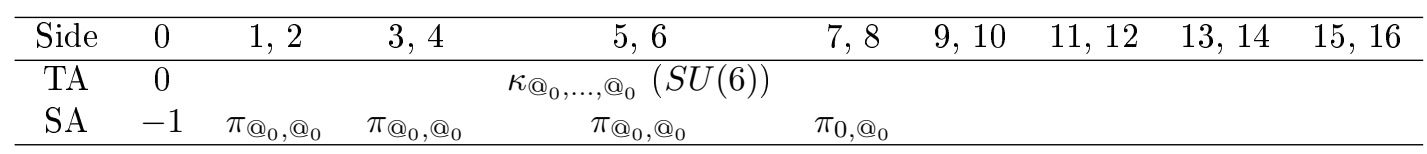

Table 49: Values, for objects, of $\iota_{3 L B}$, of $L$, and of $B$

Concept
- For free objects, the minimum magnitudes of some non-zero quantities are $\left|q_{\epsilon}\right|$ for charge and
three for $\left|\iota_{3 L B}\right|$.
- For unfree objects, the minimum magnitudes of some non-zero quantities are $\left|q_{\epsilon}\right| / 3$ for charge
and one for $\left|\iota_{3 L B}\right|$.
- Each of the quantities charge, $\iota_{3 L B}, L$, and $B$ is additive with respect to components of a
multicomponent object.

Table 50: Simple particles for which $\left|\iota_{3 C H}\right| \stackrel{=}{=}$ or $\left|\iota_{3 C H}\right| \doteq=1$ (with two columns showing some simple particles for which $\left.\left|\iota_{3 C H}\right| \underline{=} 3\right)$

\begin{tabular}{|c|c|c|c|c|c|c|}
\hline$\iota_{S}=2 S$ & $\begin{array}{c}\left|\iota_{3 C H}\right|^{\prime}=3 \\
m>0\end{array}$ & $\begin{array}{c}\left|\iota_{3 C H}\right| \stackrel{=}{=} 3 \\
m \doteq 0\end{array}$ & $\begin{array}{c}\left|\iota_{3 C H}\right|^{\prime}=2 \\
m>0\end{array}$ & $\begin{array}{c}\left|\iota_{3 C H}\right| \stackrel{=}{=} 2 \\
m \doteq 0\end{array}$ & $\begin{array}{c}\left|\iota_{3 C H}\right| \stackrel{=}{=} 1 \\
m>0\end{array}$ & $\begin{array}{c}\left|\iota_{3 C H}\right| \doteq 1 \\
m \doteq 0\end{array}$ \\
\hline 1 & $1 \mathrm{C}_{i}^{3}: 2$ & $1 \mathrm{~N}_{j}^{0}:\{1$ or 2$\}$ & $1 Q_{i}^{2}: 2$ & $1 \mathrm{R}_{i}^{0^{\prime \prime}}:\{1$ or 2$\}$ & $1 \mathrm{Q}_{j}^{1}: 2$ & $1 \mathrm{R}_{j}^{0^{\prime}}:\{1$ or 2$\}$ \\
\hline 2 & $2 W^{0}: 1$ & & $2 \mathrm{~T}^{0^{\prime \prime}}: 1$ & & $2 \mathrm{~T}^{0^{\prime}}: 1$ & \\
\hline 2 & $2 W^{3}: 2$ & & $2 \mathrm{~T}^{2}: 2$ & & $2 \mathrm{~T}^{1}: 2$ & \\
\hline
\end{tabular}

Table 51: A UMS-centric representation for the tweak bosons

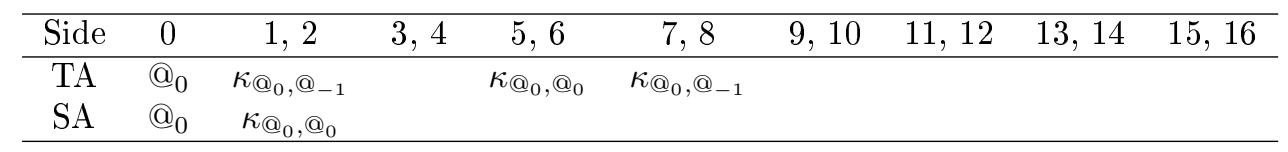

Table 52: A UMS-centric representation for the jay bosons

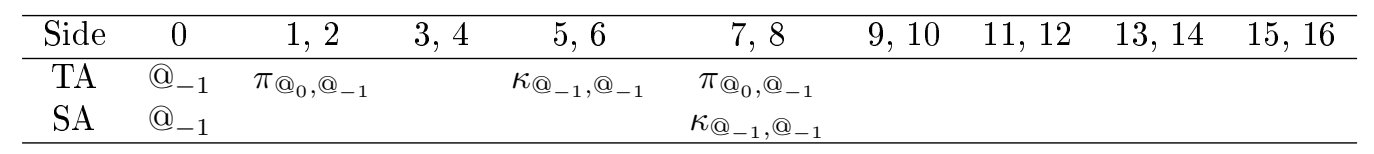

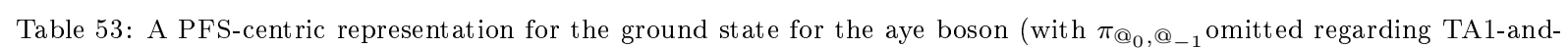
TA2 and regarding TA7-and-TA8)

\begin{tabular}{cccccccccc}
\hline Side & 0 & 1,2 & 3,4 & 5,6 & 7,8 & 9,10 & 11,12 & 13,14 & 15,16 \\
\hline TA & -1 & & & $\pi_{@_{0}, @_{-1}}$ & & & & & \\
SA & -1 & $\kappa_{@_{0}, @_{-1}}$ & & & & & & & \\
\hline
\end{tabular}




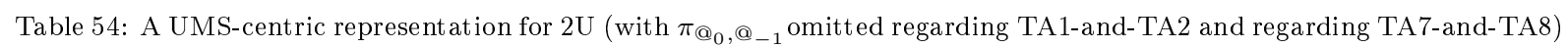

\begin{tabular}{|c|c|c|c|c|c|c|c|c|c|}
\hline Side & 0 & 1,2 & 3,4 & 5,6 & 7,8 & 9,10 & 11,12 & 13,14 & 15,16 \\
\hline $\mathrm{TA}$ & & & & ${ }_{1}, @_{-1}$ & & & & & \\
\hline SA & $@_{-1}$ & & & & & $\underbrace{}_{-1}, @$ & & & \\
\hline
\end{tabular}

Table 55: Possible correlations between U-family mathematics and simple bosons that do not belong to the U-family of root forces

\begin{tabular}{ccccccc}
\hline Subfamily & Concept & Aspect & KMS SA-side aspect & $0 \Phi$ & $0 \Phi$ mass & Residual strong force \\
\hline $2 \mathrm{U}$ & $S U(3) \uplus I$ & $I$ operator & $-\exp \left(r /\left(\left|\eta_{S A}\right|\right)\right)$ & $0 \mathrm{P}$ & $m>0$ & Attractive \\
\hline
\end{tabular}

SA9-and-SA10, see table 20a.) The representation includes a TA-side $S U(3)$ symmetry that correlates, in PFS-centric models, with oscillators TA0, TA9, and TA10. (Perhaps, regarding the seeming use of three - not two - instances of $n_{T A}=-1$ for aspects that seemingly correlate with just two oscillators, compare with the $S U(4)$ and $S U(6)$ items in table 40.) The TA-side $S U(3)$ symmetry comports with rebuttable always conservation of fermion generation. (Perhaps, see table 40.) As far as we know, each one of observations and core ongoing modeling does not correlate the (non-residual) strong interaction with either changes in elementary fermion generation or violation of CP symmetry. (Perhaps, see discussion regarding table 113.) This essay does not fully explore the extent to which this TA-side $S U(3)$ symmetry correlates with the notion of an $S U(3)$ symmetry that ongoing modeling correlates with the strong interaction. (Perhaps, note discussion regarding equation (173).) The SA-side $S U(2)$ symmetry correlates with the notion of three color charges. (See table 58a.) Discussion regarding table 69 shows, from a PFScentric perspective, modeling pertaining to the excitation and de-excitation of gluons.

We discuss the pie boson.

We explore correlations between the $2 \mathrm{U}$ solution and the pie (or, $0 \mathrm{P}$ ) boson.

Table 55 alludes to modeling that correlates the pie boson with U-family forces. Tables 26a and $26 \mathrm{~d}$ discuss the notion of $S U(3) \uplus I$. Table 55 focuses on the identity operator aspect of the notion of $S U(3) \uplus I$. Proposed modeling suggests that pie particles correlate with the ongoing modeling notion of a Yukawa potential that, in atomic nuclei, attracts hadrons to each other. (See discussion related to equation (186).) Whereas, $2 \mathrm{U}$ particle interactions correlate with color charges, 0P particles - in effect interact with clear (or, white) color charge.

Table 56 shows a PFS-centric representation for the 0P boson. The 0P simple particle does not interact with simple fermions.

The mass of the pie simple boson might approximate the masses of pions. (We base that notion on ongoing modeling - regarding nuclear physics - that features virtual pions.) This essay does not further explore modeling that might correlate with the mass of the pie boson.

\subsubsection{Some PEPM concepts re models that output the list of elementary particles}

Table 57 summarizes some PEPM concepts regarding models that output the list of elementary particles. (Perhaps, compare table 57 with table 72 )

\section{Results: properties of elementary particles and multicomponent objects}

This unit interrelates properties of elementary particles and properties of multicomponent objects.

\subsection{Summary: a table of properties of elementary particles and multicomponent objects}

Table 58 lists properties that pertain to elementary particles and that may pertain to objects that contain more than one component object. (See table 20.)

We anticipate exploring predictions about and correlations among the properties, especially in regard to properties of elementary particles.

Table 56: A PFS-centric representation for the 0P simple boson (with $\pi_{@_{0}, @_{-1}}$ omitted regarding TA1-and-TA2 and regarding TA7-and-TA8)

\begin{tabular}{cccccccccc}
\hline Side & 0 & 1,2 & 3,4 & 5,6 & 7,8 & 9,10 & 11,12 & 13,14 & 15,16 \\
\hline TA & 0 & & & & & & & & \\
SA & 0 & & & & & & & & \\
\hline
\end{tabular}


Table 57: Some PEPM concepts regarding models that output the list of elementary particles

\begin{tabular}{llll}
\hline $\begin{array}{l}\text { Type of elementary particles } \\
\text { (with respect to PDE modeling) }\end{array}$ & $\begin{array}{l}\text { PDE } \\
\text { correlation }\end{array}$ & Subfamilies & $\begin{array}{l}\text { ALG } \\
\text { correlation }\end{array}$ \\
\hline Long-range root forces & $r^{-2}$ & $\Sigma \mathrm{G}$ & $n_{T A 0}=0, n_{S A 0}=-1$ \\
Other root forces & $r^{2}$ & $2 \mathrm{U}$ & $n_{T A 0}=(-1)^{+}$ \\
Simple particles & $r^{0}$ & All others & $n_{T A 0}=n_{S A 0}$ \\
\hline
\end{tabular}

Elsewhere, we speculate that aspects of table 58a correlate with the group $S U(17)$. (See discussion related to table 20.) Elsewhere, we speculate that people might want to consider the notion that table 58 a correlates with - at least a key component of - a so-called theory of everything. (See discussion related to table 111.)

\subsection{Modeling regarding properties of elementary particles and multicomponent objects}

We discuss concepts and methods that point to results regarding some properties of elementary particles.

\subsubsection{Kinematics conservation laws}

We explore modeling regarding conservation of energy, momentum, and angular momentum.

In ongoing modeling, the electromagnetic field carries information that correlates with events that excited the field. Via de-excitations, people measure energies, momenta, and polarizations. (Also, people measure or infer that the de-excitation event features de-excitation of a mode of the electromagnetic field and does not feature de-excitation of a non-electromagnetic field.) People infer information about excitation events.

We want to discuss the extent to which proposed modeling models for $\Sigma \mathrm{G}$ (or, G-family) fields reflect encoded information.

We start by exploring modeling related to energy, momentum, and angular momentum.

Ongoing modeling discusses models for objects, internal properties (such as spin and charge) of objects, motion-centric properties (such as momentum) of objects, and interactions (or, forces) that affect internal properties of objects or motions of objects.

We discuss symmetries that ongoing modeling and proposed modeling correlate with conservation laws related to motion.

Table 59 summarizes symmetries correlating with kinematics conservation laws. Ongoing modeling correlates an $S 1 G$ symmetry with conservation of energy. The one-element term $S 1 G$ denotes a symmetry correlating with a group for which one generator pertains. Core proposed modeling PFS modeling pertains to the existence of elementary particles and multicomponent objects and pertains to interactions between elementary particles and multicomponent objects. Supplementary proposed modeling includes KMS modeling for motion. Supplementary proposed modeling KMS modeling for motion includes the kinematics symmetries that table 58 shows.

The following concepts pertain regarding proposed modeling.

- Models for the kinematics of objects in free environments need to include the possibility that all three conservation laws pertain. The relevance of all three conservation laws correlates with modeling that correlates with the notion of a distinguishable object and with the notion of a free environment.

- Objects can exist as components of, let us call them, larger objects that are free. For one example, an electron can exist as part of an atom. For another example, a hadron can exist as part of an atomic nucleus that includes more than one hadron. The two-word term confined environment can pertain.

- Models regarding the kinematics (or, dynamics) of objects in unfree (or, confined) environments do not necessarily need to embrace all three kinematics conservation laws. Unfree objects can model as existing in the contexts of larger free objects.

- For a proposed modeling ALG model to embrace conservation of momentum and conservation of angular momentum, one, in effect, adds (to a model for an object) four SA-side oscillators and expresses two instances of $S U(2)$ symmetry. Double-entry bookkeeping suggests adding four TAside oscillators. Proposed modeling suggests that, for each of the eight added oscillators, $n=n_{T A 0}$. 
Table 58: A catalog of properties that pertain to elementary particles and that may pertain to multicomponent objects

(a) The catalog (with DoF abbreviating the three-word phrase degrees of freedom)

\begin{tabular}{|c|c|c|c|c|}
\hline$\overline{\mathrm{XA}}$ & $\begin{array}{l}\text { SA-side } \\
\text { symmetry } \\
\text { (* denotes } \\
\text { SA-side and } \\
\text { TA-side) }\end{array}$ & Properties & $\begin{array}{l}\text { SA-side } \\
\text { count }\end{array}$ & $\begin{array}{l}\text { TA-side } \\
\text { symmetry } \\
\text { (summable } \\
\text { and } \\
\text { conserved) }\end{array}$ \\
\hline 0 & - & Positive or zerolike rest energy & - & - \\
\hline 1,2 & $S U(2)$ & Charge & $3(-, 0,+)$ & $U(1)$ \\
\hline 3,$4 ; 5,6$ & $S U(4)$ & Rest energy minus freeable energy & - & $U(1)$ \\
\hline 3,4 & $S U(2) \times U(1) *$ & Rest energy. Isomers (See table 58c ) & 6 & - \\
\hline 5,6 & $(S U(2))$ & Freeable energy (Gens, re simple fermions) & (3 gens) & $(-)$ \\
\hline 7,8 & $S U(2)$ & 3LB number & $3(-, 0,+)$ & $U(1)$ \\
\hline 9,10 & $S U(2)$ & Color charge & $3(\mathrm{r}, \mathrm{b}, \mathrm{g})$ & - \\
\hline 11,12 & $S U(2)$ & Momentum & $3 \mathrm{DoF}$ & $U(1)$ \\
\hline 13,14 & $S U(2)$ & Angular momentum & $3 \mathrm{DoF}$ & $U(1)$ \\
\hline 15,16 & $S U(2) \times U(1) *$ & Isomers (See table 58c.) & 6 & - \\
\hline
\end{tabular}

(b) Notes about columns that pertain for the catalog

Note

- The column labeled XA displays sets of oscillators. Here, XA includes each of TA and SA.

- The column labeled SA-side symmetry (generally, with two marked exceptions) lists SA-side symmetries. The symbol * correlates with an exception. For the exceptions, the column combines SA-side and TA-side symmetries.

- The column labeled properties lists properties.

- Numbers in the column labeled SA-side count equal the number of generators for the groups in the column labeled SA-side symmetry.

- For a row for which the column labeled TA-side symmetry shows the group $U(1)$, the property is a conserved quantity and the property sums across components of a multicomponent object.

(c) Notes about rows and items that pertain for the catalog

Note
- The notion of zerolike rest energy pertains for some elementary particles and not for other
objects.

- Each object has a charge. The charge is an integer multiple of one-third the magnitude of the charge of an electron. The symbol $(-, 0,+)$ correlates with the following three possibilities. The integer is negative. The integer is zero. The integer is positive.

- For an object that remains intact during an interaction with other objects, the quantity rest energy minus freeable energy remains unchanged by the interaction. The pairs XA3-and-XA4 correlate with rest energy. The pairs XA5-and-XA6 correlate with freeable energy.

- The row for which the XA column shows just the integers three and four is a sub-case of the row immediately above that row.

- The row for which the XA column shows just the integers five and six is a sub-case of the row two rows above that row.

- The one-element item gens abbreviates the word generations. The notion of generations pertains for elementary fermions only.

- Each object has a 3LB number. The 3LB number is an integer multiple of the magnitude of the baryon number for a quark. The symbol $(-, 0,+)$ correlates with the following three possibilities.

The integer is negative. The integer is zero. The integer is positive.

- As far as we know, other permuting, among rows, of the items that table 58a shows as correlating with XA9-and-XA10 through XA15-and-XA16 would not make a difference regarding modeling that this essay discusses.

- The three-element item (r, b, g) correlates with three color charges - red, blue, and green.

- The one-element item DoF abbreviates the three-word phrase degrees of freedom.

- Each of the two notions of six isomers might correlate with a physics-relevant concept. (See table 21a.) The notion that correlates with SM6a might correlate with six isomers of charge (and of simple particles), with PR6ISP modeling, and with explanations for observed ratios of dark matter effects to ordinary matter effects. (See table $21 \mathrm{~b}$.) 
Table 59: Symmetries correlating with kinematics conservation laws

\begin{tabular}{|c|c|c|c|c|c|}
\hline \multirow[t]{2}{*}{ Conservation law } & \multirow[t]{2}{*}{$\begin{array}{l}\text { Ongoing } \\
\text { modeling } \\
\text { KMS models }\end{array}$} & \multicolumn{2}{|c|}{$\begin{array}{l}\text { Core proposed } \\
\text { modeling } \\
\text { PFS models }\end{array}$} & \multicolumn{2}{|c|}{$\begin{array}{l}\text { Proposed } \\
\text { modeling } \\
\text { UMS/KMS models }\end{array}$} \\
\hline & & TA-side & SA-side & TA-side & SA-side \\
\hline Conservation of energy & $S 1 G$ & $S U(5)$ & & $U(1)$ or $S 1 G$ & $S U(4)$ \\
\hline Conservation of momentum & $S U(2)$ & & $S U(2)$ & $U(1)$ & $S U(2)$ \\
\hline Conservation of angular momentum & $S U(2)$ & & $S U(2)$ & $U(1)$ & $S U(2)$ \\
\hline
\end{tabular}

Table 60: Possible representation for conservation of energy, momentum, and angular momentum for free objects

(a) The case $n_{T A 0}=0$ (with $\kappa_{0,0,0,0,0}$ spanning the three items showing the symbol *)

\begin{tabular}{cccccccccc}
\hline Side & 0 & 1,2 & 3,4 & 5,6 & 7,8 & 9,10 & 11,12 & 13,14 & 15,16 \\
\hline TA & $*$ & & & & & & $*$ & $*$ & \\
SA & 0 & & & & & & $\kappa_{0,0}$ & $\kappa_{0,0}$ & \\
\hline
\end{tabular}

(b) The case $n_{T A 0}=-1$ (with $\kappa_{-1,-1,-1,-1,-1}$ spanning the three items showing the symbol *)

\begin{tabular}{ccccccccccc}
\hline Side & 0 & 1,2 & 3,4 & 5,6 & 7,8 & 9,10 & 11,12 & 13,14 & 15,16 \\
\hline TA & $*$ & & & & & & $*$ & $*$ & \\
SA & -1 & & & & & & $\kappa_{-1,-1}$ & $\kappa_{-1,-1}$ & \\
\hline
\end{tabular}

- For some modeling, proposed modeling suggests combining the four TA-side oscillators with the TA0 oscillator to correlate with an $S U(5)$ symmetry. For such modeling, proposed modeling suggests that the TA-side $S U(5)$ symmetry correlates with conservation of energy. (See table 59.)

- For some modeling, it might be appropriate to use $S U(4)$ plus $S 1 G$.

- For some modeling, it might be appropriate to use $S U(4)$ plus $U(1)$.

- In proposed modeling, each of $S 1 G$ and $U(1)$ can correlate with one harmonic oscillator. (See table 13.) In ongoing modeling, $S 1 G$ correlates with one dimension with respect to spacetime coordinates. This essay de-emphasizes discussing details of mathematical relationships between $S 1 G$ and $U(1)$.

- Table 60 shows possible proposed modeling representations of kinematics conservation laws for free objects. We think that it might not be necessary to designate specific oscillator pairs. (A choice of oscillator pairs XA11-and-XA12 and XA13-and-XA14 correlates with other modeling uses for oscillators XA0-through-XA10. A choice of oscillator pairs XA11-and-XA12 and XA13-and-XA14 correlates with the modeling that table 58a shows.) For - at least - convenience regarding notation, this essay chooses the oscillator pairs XA11-and-XA12 and XA13-and-XA14. (For representations for the case of $\Sigma_{\max }=20$, one might want to use oscillator pairs such as XA21-and-XA22 and XA23-and-XA24. Presumably, the pair XA19-and-XA20 would correlate with 10G.)

- Special relativity correlates with boost symmetry, which correlates with an additional $S U(2)$ symmetry. Boost symmetry correlates with KMS modeling. Core proposed modeling does not necessarily need to accommodate boost symmetry.

- A contrast between table 48 and table 60 pertains. Some information in table 48 correlates with symmetries and conservation laws (or with approximate symmetries and rebuttable somewhat conservation laws) that pertain regarding fields and quantum excitations. Some information in table 60 correlates with interactions and with conservation laws that pertain regarding kinematics.

- The following proposed modeling aspects can pertain regarding combining two free objects to form one free object.

- Each of the two original objects contributes two SA-side $S U(2)$ symmetries. 
- Two of the original SA-side $S U(2)$ symmetries can pertain regarding modeling for the motion of the new object. The other two SA-side $S U(2)$ symmetries are available for modeling internal aspects of the new object. Neither of the original two objects continues to exhibit both conservation of momentum and conservation of angular momentum. For example, for a system consisting of a star and a planet, neither the star nor the planet exhibits conservation of momentum. In this context, kinematics modeling for each of the two original objects can correlate with unfree modeling. In this context, each leftover internal $S U(2)$ symmetry can correlate with modeling for one of the original objects, for fields that model interactions between the two original objects, for combinations of objects and fields, or for something else. Here, the notion of something else can correlate with, for example, aspects of two-body modeling that features the concept of reduced mass.

- Similarly, one of the original two TA-side $S U(5)$ symmetries can pertain regarding modeling for the motion of the new object. The other TA-side $S U(5)$ symmetry is available for modeling internal aspects of the new object.

\subsubsection{G-family phenomena, including electromagnetism and gravity}

We explore aspects regarding G-family forces and regarding components of G-family forces.

In ongoing modeling KMS modeling, an excitation of a G-family force carries information through which people infer aspects of an event that includes the excitation. For example, people measure the energy of a photon and might use that information to infer information about an atomic transition that excited the photon.

We explore PFS modeling that encodes, regarding $2 \mathrm{G}$ modes, information about excitations of the overall $2 \mathrm{G}$ field. We anticipate that PFS modeling points to encoded information to which ongoing modeling KMS modeling does not point. The additional encoded information correlates with the socalled isomer or isomers that participated in the creation of the photon. (See table 58. See discussion leading to table 88. Perhaps note, for example, discussion - pertaining to isomers and spans - related to equation 149 and discussion related to table 93.)

We consider the left circular polarized mode. Modeling for some excitations correlates with aspects of table 35 .

We consider an excitation that models conceptually as combining an excitation of the left circular mode of $4 \mathrm{G}$ and the right circular mode of $2 \mathrm{G}$. (This essay de-emphasizes the possible relevance of an actual object that combines a graviton and a photon.) The combination yields a left circular polarization spin-1 excitation. The combination correlates with $2 \mathrm{G}$.

Equation (65) provides notation that we use for such combinations. The symbol $\Sigma G$ denotes a subfamily of the G-family of solutions to equation (38). The symbol $\Gamma$ denotes a set of even integers selected from the set $\{2,4,6,8\}$. We use the symbol $\lambda$ to denote an element of $\Gamma$. Each value of $\lambda$ correlates with the oscillator pair $\mathrm{SA}(\lambda-1)$-and-SA $\lambda$. (Elsewhere, we discuss aspects correlating with the limit $\lambda \leq 8$. See discussion related to table 62.) For the above example of subtracting spin- 1 from spin-2, the notation $\Gamma=24$ pertains and equation 66 pertains.

$\Sigma \mathrm{G} \Gamma$

$$
\Sigma=|-2+4|=2
$$

Table 61 points to possibly relevant solutions. The label monopole correlates with the existence of one mathematical solution for each item in the column labeled monopole. The label dipole correlates with the existence of two mathematical solutions for each item in the column labeled dipole. For example, for $\Gamma=24$, each one of the solutions 2 G24 and 6G24 pertains. The symbol 6 G24 correlates with $\Sigma=|+2+4|=6$. The label quadrupole correlates with the existence of four mathematical solutions for each item in the column labeled quadrupole. G-family physics does not include phenomena that might correlate with the symbol 0G. For each of two quadrupole items, the one 0G mathematical solution is not relevant to G-family physics. (The solutions may be relevant to physics other than G-family physics. See, for example, table 73.) For example, the solution 0G246, which correlates with $|-2-4+6|$, is not relevant to G-family physics. The label octupole correlates with the existence of eight mathematical solutions for the one item in the column labeled octupole. The solution 0G2468 is not relevant to G-family physics. The table notes a conceptually possible $0 \mathrm{G} \emptyset$ solution. The symbol $\emptyset$ denotes the empty set.

So far, our discussion of the terms monopole through octupole features numbers of solutions and does not feature physics phenomena. 
Table 61: G-family solutions that may be relevant

\begin{tabular}{ccccc}
\hline Other & Monopole & Dipole & Quadrupole & Octupole \\
\hline 0G $\emptyset$ & $2 \mathrm{G} 2$ & $\Sigma \mathrm{G} 24$ & $\Sigma \mathrm{G} 246$ & $\Sigma \mathrm{G} 2468$ \\
& $4 \mathrm{G} 4$ & $\Sigma \mathrm{G} 26$ & $\Sigma \mathrm{G} 248$ & \\
& $6 \mathrm{G} 6$ & $\Sigma \mathrm{G} 28$ & $\Sigma \mathrm{G} 268$ & \\
& $8 \mathrm{G} 8$ & $\Sigma \mathrm{G} 46$ & $\Sigma \mathrm{G} 468$ & \\
& & & & \\
& & & & \\
& & & \\
\end{tabular}

So far, proposed modeling does not depend on choosing a kinematics model. Examples of kinematics models include Newtonian physics and general relativity.

We posit that the words monopole through octupole correlate, for ongoing modeling KMS Newtonian modeling, with force laws. Ongoing modeling correlates the word monopole with a potential energy that varies as $r^{-1}$ and with the RSDF of $r^{-2}$. (The concept of RSDF correlates with KMS modeling.) Here, $r$ denotes the distance from the center of the one relevant object. RSDF abbreviates the five-word term radial spatial dependence of force. Here, we de-emphasize angular aspects of forces. (Discussion related to table 66 shows relationships between some solutions that table 61 lists and aspects of ongoing modeling. For example, 2G2 correlates with interactions with charge. 2G24 correlates with interactions with nominal magnetic dipole moment.)

Table 62 shows representations for the G-family solutions that table 61 lists. The solutions correlate with symmetries pertaining to ground states. For the case of $\Sigma$ being two, excitations comport with the type of $\Sigma \mathrm{G}$ excitations to which table 36 alludes. For the cases of $\Sigma$ being four, six, or eight, excitations comport with the type of $\Sigma \mathrm{G}$ excitations to which table 48 alludes. In table 62 , the rightmost seven columns comport with double-entry bookkeeping. For example, a TA-side $S U(3)$ symmetry alludes to two additional TA-side oscillators for each of which $n_{T A}=0$. Those two oscillators plus the TA0 oscillator correlate with $\kappa_{0,0,0}$ (or, with $S U(3)$ symmetry). The symbol $\mathrm{A} 0+$ correlates with an oscillator pair for which, for each of the two oscillators, the symbol $@_{0}$ pertains. (Perhaps, see table 13.) In table $62 \mathrm{a}$, the column regarding span pertains regarding aspects of dark matter specifically and, generally, aspects of astrophysics and cosmology. (See table 93 and table $62 \mathrm{~b}$.) Regarding each $\Sigma>0$ solution that the table shows, the KMS radial behavior of the potential is $r^{n_{S A 0}}$. The RSDF is $r^{n_{S A 0}-1}$.

Table 63 generalizes from table $62 \mathrm{~b}$.

Table 64 lists notions that might correlate with a limit of $\lambda \leq 8$. Possibly, each one of the notions is relevant.

Table 65 lists G-family solutions $\Sigma$ G $\Gamma$ for which both $\Sigma$ does not exceed eight and $\Sigma$ appears in the list $\Gamma$. The expressions $|-2+4-6+8|$ and $|-2-4-6+8|$ show that two solutions comport with the notion of $4 \mathrm{G} 2468$. The expressions $|+2+4-6+8|$ and $|-2-4+6+8|$ show that two solutions comport with the notion of $8 \mathrm{G} 2468$. We use the symbol $\Sigma \gamma$ to refer to the set of G-family solutions $\Sigma G \Gamma$ for which $\Sigma$ appears in the list $\Gamma$. (See equation (67).) We use the symbol $\gamma \lambda$ to refer to the set of G-family solutions $\Sigma \mathrm{G} \Gamma$ for which $\lambda$ appears in the list $\bar{\Gamma}$ and $\Sigma$ does not appear in the list $\Gamma$. (See equation (68).)

$$
\begin{gathered}
\Sigma \gamma=\{\Sigma \mathrm{G} \Gamma \mid \Sigma \in \Gamma\} \\
\gamma \lambda=\{\Sigma \mathrm{G} \Gamma \mid \lambda \in \Gamma, \Sigma \notin \Gamma\}
\end{gathered}
$$

Proposed modeling correlates the two-word term monopole gravity (or, the four-word term monopole component of gravity) with the $4 \mathrm{G} 4$ solution. (See table 63.) Proposed modeling correlates the twoelement term non-monopole gravity with the 4G48, 4G246, 4G2468a, and 4G2468b solutions. Solution $4 \mathrm{G} 48$ correlates with some effects for which ongoing modeling might use the four-word term dark energy negative pressure. Solutions 4G2468a and 4G2468b correlate with some effects for which ongoing modeling uses the three-word term inflationary dark energy. Each one of solutions 4G48, 4G246, 4G2468a, and $4 \mathrm{G} 2468$ b correlates also with - regarding ongoing modeling - effects for which people do not use either one of the terms dark energy negative pressure and inflationary dark energy.

Table 66 discusses aspects of table 65. Here, we anticipate possibilities for developing supplementary proposed modeling kinematics models. (See, for example, discussion related to table 119 and discussion related to table 123.) Here, we use wording that correlates with KMS classical physics Newtonian modeling. Solution 2G248 correlates with interactions with an object for which a non-zero magnetic dipole moment pertains, an axis of rotation pertains regarding the orientation of the axis of the magnetic 
Table 62: PFS information, including TA-side symmetries, regarding G-family solutions

(a) $\Sigma \Phi \Gamma$, TA-side symmetries, and other aspects

\begin{tabular}{|c|c|c|c|c|c|c|c|c|}
\hline$\Sigma \Phi \Gamma$ & $\begin{array}{c}\text { Span } \\
\text { (for } \\
\iota_{I} \geq 6 \text { ) }\end{array}$ & $\begin{array}{c}\text { TA-side } \\
S U\left(\_\right) \\
\text {symmetry }\end{array}$ & $\begin{array}{c}\text { TA } \\
0\end{array}$ & $\begin{array}{c}\text { SA } \\
0\end{array}$ & $\begin{array}{c}\mathrm{SA} \\
1 \text { and } 2\end{array}$ & $\begin{array}{c}\mathrm{SA} \\
3 \text { and } 4\end{array}$ & $\begin{array}{c}\mathrm{SA} \\
5 \text { and } 6\end{array}$ & $\begin{array}{c}\text { SA } \\
7 \text { and } 8\end{array}$ \\
\hline $0 \mathrm{G} \emptyset$ & 1 & None & -1 & -1 & & & & \\
\hline $2 \mathrm{G} 2$ & 1 & None & 0 & -1 & $\pi_{0, @_{0}}$ & & & \\
\hline $4 \mathrm{G} 4$ & 6 & $S U(3)$ & 0 & -1 & $\mathrm{~A} 0+$ & $\pi_{0, @_{0}}$ & & \\
\hline$\Sigma \mathrm{G} 24$ & 1 & None & 0 & -2 & $\pi_{0, @_{0}}$ & $\pi_{0, @_{0}}$ & & \\
\hline 6G6 & 2 & $S U(5)$ & 0 & -1 & $\mathrm{~A} 0+$ & $\mathrm{A} 0+$ & $\pi_{0, @_{0}}$ & \\
\hline$\Sigma \mathrm{G} 26$ & 6 & $S U(3)$ & 0 & -2 & $\pi_{0, @_{0}}$ & $\mathrm{~A} 0+$ & $\pi_{0, @_{0}}$ & \\
\hline$\Sigma \mathrm{G} 46$ & 6 & $S U(3)$ & 0 & -2 & $\mathrm{~A} 0+$ & $\pi_{0, @_{0}}$ & $\pi_{0, @_{0}}$ & \\
\hline$\Sigma \mathrm{G} 246$ & 1 & None & 0 & -3 & $\pi_{0, @_{0}}$ & $\pi_{0, @_{0}}$ & $\pi_{0, @_{0}}$ & \\
\hline 8G8 & 1 & $S U(7)$ & 0 & -1 & $\mathrm{~A} 0+$ & $\mathrm{A} 0+$ & $\mathrm{A} 0+$ & $\pi_{0, @_{0}}$ \\
\hline$\Sigma \mathrm{G} 28$ & 2 & $S U(5)$ & 0 & -2 & $\pi_{0, @_{0}}$ & $\mathrm{~A} 0+$ & $\mathrm{A} 0+$ & $\pi_{0, @_{0}}$ \\
\hline$\Sigma \mathrm{G} 48$ & 2 & $S U(5)$ & 0 & -2 & $\mathrm{~A} 0+$ & $\pi_{0, @_{0}}$ & $\mathrm{~A} 0+$ & $\pi_{0, @_{0}}$ \\
\hline$\Sigma G 68$ & 2 & $S U(5)$ & 0 & -2 & $\mathrm{~A} 0+$ & $\mathrm{A} 0+$ & $\pi_{0, @_{0}}$ & $\pi_{0, @_{0}}$ \\
\hline$\Sigma \mathrm{G} 248$ & 6 & $S U(3)$ & 0 & -3 & $\pi_{0, @_{0}}$ & $\pi_{0, @_{0}}$ & $\mathrm{~A} 0+$ & $\pi_{0, @_{0}}$ \\
\hline$\Sigma G 268$ & 6 & $S U(3)$ & 0 & -3 & $\pi_{0, @_{0}}$ & $\mathrm{~A} 0+$ & $\pi_{0, @_{0}}$ & $\pi_{0, @_{0}}$ \\
\hline$\Sigma \mathrm{G} 468$ & 6 & $S U(3)$ & 0 & -3 & $\mathrm{~A} 0+$ & $\pi_{0, @_{0}}$ & $\pi_{0, @_{0}}$ & $\pi_{0, @_{0}}$ \\
\hline$\Sigma \mathrm{G} 2468$ & 1 & None & 0 & -4 & $\pi_{0, @_{0}}$ & $\pi_{0, @_{0}}$ & $\pi_{0, @_{0}}$ & $\pi_{0, @_{0}}$ \\
\hline
\end{tabular}

(b) Notes regarding excitations and regarding information that correlates with specific $\Sigma \mathrm{G} \Gamma$

Note

- An excitation of a $\Sigma$ G field does not (directly) encode information about a relevant $\Sigma G \Gamma$.

- Proposed modeling includes so-called PR $\iota_{I} \mathrm{ISP}$ modeling, with $\iota_{I}$ being one of the integers one, six, and 36. The models address aspects of astrophysics and aspects of cosmology. The integer $\iota_{I}$ denotes a number of so-called isomers of simple particles.

- In this respect, PR1ISP modeling correlates with core ongoing modeling. The notion of span is not relevant. (Or, one can say that each simple particle and each component of root forces has a span of one.)

- For PR $\iota_{I}$ ISP modeling for which $\iota_{I} \geq 6$, an excitation (for example, of a $\Sigma G$ field) encodes information that specifies relevant isomers of simple particles. Here, the word relevant denotes relevant to the excitation. The word span denotes the number of relevant isomers.

- For PR $\iota_{I}$ ISP modeling for which $\iota_{I} \geq 6$, a de-excitation must correlate with an isomer in the list of isomers that correlates with the relevant excitation.

Table 63: Notes regarding excitations and regarding information that correlates with excitations, plus notes regarding PFS modeling and KMS modeling

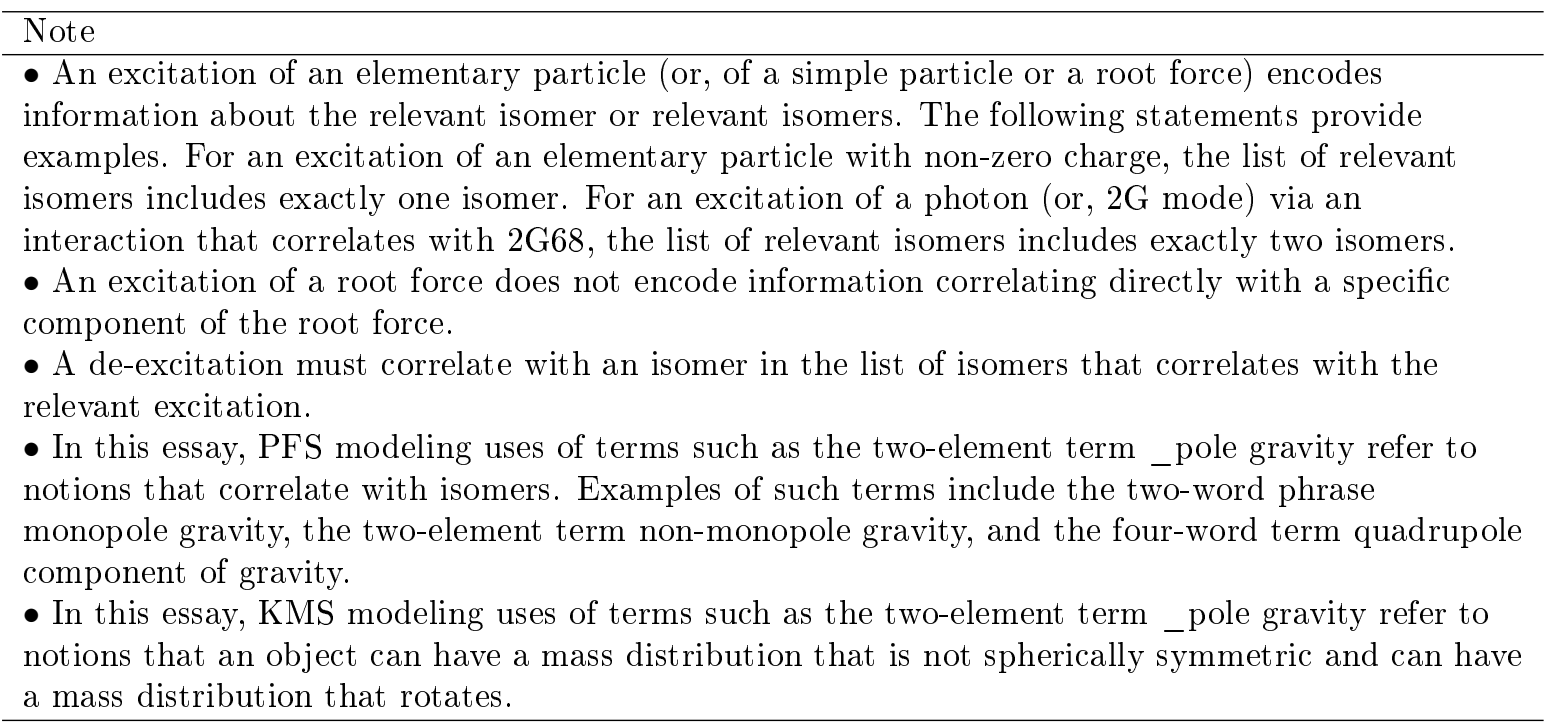


Table 64: Notions that might correlate with a limit of $\lambda \leq 8$

Notion
- The limit might correlate with a scaling law. For the $\Gamma$ of $2468 \llbracket 10 \rrbracket$, the one-element phrase
hexadecimal-pole would pertain. Here, the symbol $\llbracket 10 \rrbracket$ denotes the number ten. Assuming KMS
Newtonian modeling, the RSDF (or, radial spatial dependence of force) would be $r^{-6}$. We consider
interactions between two similar, neighboring, non-overlapping, somewhat spherically symmetric
objects. A $\Sigma G 2468 \llbracket 10 \rrbracket$ force would scale like $\left(v^{3} \rho\right)^{2} /(v r)^{6}$, in which $v$ is a non-dimensional scaling
factor that correlates with linear size (or, a length), $\rho$ is the relevant object property for the case
for which $v=1$, and $r$ is the distance between the centers of the objects. The factor $v^{3}$ provides
for scaling for an object that has three spatial dimensions. The force would be independent of $v$.
That independence might suggest, from a standpoint of physics, that $\varrho=0$ pertains.
- The limit might correlate with the notion of three eras in the rate of expansion of the universe.
(See discussion related to table 102 ) Proposed modeling correlates those eras with (respectively,
working backwards in time from the present era) dipole repulsion, quadrupole attraction, and
octupole repulsion. We know of no evidence for an era that would correlate with hexadecimal
phenomena.
• The limit might correlate with a TA-side $S U(9)$ symmetry. Based on thinking that leads to table
$62,10 G \llbracket 10 \rrbracket$ correlates with a TA-side $S U(9)$ symmetry. We posit that remarks regarding equation
(46) pertain. Here, we de-emphasize the notion that $16 \mathrm{G} \llbracket 16 \rrbracket$ has relevance to physics. (See
discussion related to table 123 .) The solution $16 \mathrm{G} \llbracket 16 \rrbracket$ would correlate with TA-side $S U(17)$
symmetry.
$\bullet$ The limit might correlate with the notion of channels. Discussion related to equation (135)
suggests that a that exceeds eight is not relevant regarding G-family physics.
- The limit might correlate with modeling that correlates with aspects of table 20 . This essay
de-emphasizes this possibility.

Table 65: $\Sigma \gamma$ solutions (or, G-family solutions for which $\Sigma$ appears in the list $\Gamma$ )

\begin{tabular}{ccccc}
\hline$\Sigma$ & Monopole & Dipole & Quadrupole & Octupole \\
\hline 2 & $2 \mathrm{G} 2$ & $2 \mathrm{G} 24$ & $2 \mathrm{G} 248$ & \\
4 & $4 \mathrm{G} 4$ & $4 \mathrm{G} 48$ & $4 \mathrm{G} 246$ & $4 \mathrm{G} 2468 \mathrm{a}, 4 \mathrm{G} 2468 \mathrm{~b}$ \\
6 & $6 \mathrm{G} 6$ & & $6 \mathrm{G} 468$ & \\
8 & $8 \mathrm{G} 8$ & & & $8 \mathrm{G} 2468 \mathrm{a}, 8 \mathrm{G} 2468 \mathrm{~b}$ \\
\hline
\end{tabular}


Table 66: KMS-modeling interpretations regarding some $\Sigma \gamma$ force components for which $\Sigma \leq 4$

(a) Interactions

\begin{tabular}{cl}
\hline Components & Interactions with ... \\
\hline $2 \mathrm{G} 2$ & Charge \\
$2 \mathrm{G} 24$ & Magnetic dipole moment \\
$2 \mathrm{G} 248$ & Magnetic dipole moment for which the axis changes over time \\
$4 \mathrm{G} 4$ & Mass \\
$4 \mathrm{G} 48$ & Mass that rotates \\
$4 \mathrm{G} 246$ & Quadrupole moment of mass \\
$4 \mathrm{G} 2468 \mathrm{a}, 4 \mathrm{G} 2468 \mathrm{~b}$ & Quadrupole moments of mass that rotates \\
\hline
\end{tabular}

(b) An interpretation

\begin{tabular}{cl}
\hline Aspect & Interpretation \\
\hline $8 \in \Gamma$ & Rotation \\
\hline
\end{tabular}

dipole moment, and the axis of rotation does not match the axis correlating with the magnetic dipole moment. The notion of a vector cross product between a vector correlating with the axis of dipole moment and a vector correlating with the axis of rotation pertains. For the earth, the 2G248 interaction correlates with the non-alignment of the axis of rotation and the axis of the magnetic field. We posit that $8 \in \Gamma$ - or the number eight appearing in the list $\Gamma$ - correlates with non-zero rotation. One of $4 \mathrm{G} 2468 \mathrm{a}$ and $4 \mathrm{G} 2468 \mathrm{~b}$ interacts - with rotational aspects of quadrupole distributions of mass - based on an axis of maximal moment of inertia. The other one of $4 \mathrm{G} 2468 \mathrm{a}$ and $4 \mathrm{G} 2468 \mathrm{~b}$ interacts - with rotational aspects of quadrupole distributions of mass - based on an axis of minimal moment of inertia.

Statements above regarding $2 \mathrm{G}$ and $4 \mathrm{G}$ correlate with concepts that equations $(69)$ and $(70)$ symbolize. PFS modeling regarding quantum states and excitations does not necessarily involve modeling pertaining to translational motion. Equation (69) pertains. (See table 48.) Equation (70) correlates with linking Gfamily physics to models for forces and translational motion. (See discussion above regarding $2 \mathrm{G}$ and $4 \mathrm{G}$ and see, for example, table 62.) Another aspect of such linking correlates with kinematics conservation laws. (See discussion related to table 59.)

$$
\Sigma \mathrm{G} \leftrightarrow \text { quantum excitations }
$$

$\Sigma \mathrm{G} \Gamma \leftrightarrow$ a bridge between quantum excitations and kinematics forces

We explore the extent to which components of G-family forces interact with simple particles. (This exploration correlates with PFS modeling.)

We combine aspects of equation (46), table 59, and table 62. We posit that TA-side aspects of table 59 and TA-side aspects of table 62 combine. For example, for 8G8, a TA-side $S U(11)$ symmetry would pertain. (In table 59, seven TA-side oscillators pertain. In table 62, five TA-side oscillators pertain. The tables share their respective $n_{T A 0}=0$ values. Seven plus five minus one is 11.) For example, for $4 \mathrm{G} 4$, a TA-side $S U(7)$ symmetry would pertain. For example, for $2 \mathrm{G} 2$ or $2 \mathrm{G} 24$, a TA-side $S U(5)$ symmetry would pertain. We posit a limit that correlates with aspects of equation (46). We posit that each component that appears in table 62 and has a TA-side symmetry of None or $S U(3)$ can interact with simple particles. (Here, combining the TA-side symmetry that table 62 shows with the conservation of energy symmetry produces, respectively, $S U(5)$ or $S U(7)$.) We posit that components that appear in table 62 and have a TA-side symmetry of None or $S U(3)$ can interact with multicomponent objects. We posit that each component that appears in table 62 and has a TA-side symmetry of $S U(5)$ or $S U(7)$ does not interact with simple particles. (Here, combining the TA-side symmetry that table 62 shows with the conservation of energy symmetry produces, respectively, $S U(9)$ or $S U(11)$.) We posit that a combined symmetry of either $S U(9)$ or $S U(11)$ correlates with possible interactions with multicomponent objects.

For example, 2G68 can interact with an atom but not with an isolated electron. (Table 62 shows, regarding 2G68, a TA-side $S U(5)$ symmetry.) We correlate 2 G68 with at least the 21-centimeter hyperfine interaction with hydrogen atoms. (See discussion related to equation (162).) Generally, $6 \in \lambda$ can correlate with interactions regarding freeable energies of objects. (See discussion related to table 25.) Generally, $8 \in \lambda$ can correlate with interactions regarding rotations of objects or spins of objects. (See discussion related to table 25 and see table $66 \mathrm{~b}$.) 
Table 67: G-family solutions for which $\lambda \leq 4$

\begin{tabular}{ccc}
\hline Other & Monopole & Dipole \\
\hline 0G $\emptyset$ & $2 \mathrm{G} 2$ & $\Sigma \mathrm{G} 24$ \\
& $4 \mathrm{G} 4$ & \\
\hline
\end{tabular}

We contrast aspects of proposed modeling G-family modeling with a possible proposed modeling interpretation of aspects of ongoing modeling.

Table 67 contrasts with table 61 . Regarding table 67, we deploy PEPM techniques, but with an assumption that the maximum $\lambda$ in any $\Gamma$ is 4 . Ongoing modeling does not necessarily include a particle that would correlate with the $0 \mathrm{G} \emptyset$ solution. Ongoing modeling includes classical physics modeling and quantum physics modeling that correlate with $2 \mathrm{G} 2$ and $2 \mathrm{G} 24$. Ongoing modeling does not directly include notions that would correlate with 6G24. Ongoing modeling includes classical physics models for gravity but does not (yet) include a complete statement regarding a graviton (or, regarding a quantum mechanical treatment correlating with $4 \mathrm{G} 4)$.

\subsubsection{Conservation of lepton number minus baryon number}

We explore the notion of conservation of lepton number minus baryon number.

Equation (71) shows a quantity, $N_{L-B}$ (or, lepton number minus baryon number). The symbol $L$ correlates with the ongoing modeling notion of lepton number. The symbol $B$ correlates with the ongoing modeling notion of baryon number. For a matter lepton, $L=+1$ and $B=0$. For an antimatter lepton, $L=-1$ and $B=0$. For a matter quark, $L=0$ and $B=1 / 3$. For an antimatter quark, $L=0$ and $B=-1 / 3$. Other than possibly for charged T-family bosons, for simple bosons and root forces, $0=L=B=N_{L-B}$. In ongoing modeling, $N_{L-B}$ is a conserved quantity. Equation (71) defines the symbol $\iota_{3 L B}$.

$$
N_{L-B}=L-B \text { and } \iota_{3 L B}=3\left(N_{L-B}\right)
$$

We correlate, with $\iota_{3 L B}$, the two-element term 3LB number. Sometimes, we use the one-element term 3LB to denote 3LB number. The four-element term conservation of 3LB number pertains.

Proposed modeling includes the notion of conservation of $\iota_{3 L B}$. (See, for example, table 58.)

Each of equations $72,(73), 74)$, and $(75)$ shows an interaction that would involve the $2 \mathrm{~T}^{+1}$ simple particle; transform a matter quark into another simple fermion; and conserve $\iota_{3 L B}, L$, and $B$. Here, for fermions, the notation $1 \Phi_{\iota_{3 L B} \iota_{3 L, 3 B}}^{\iota_{3 C H}}$ pertains. Here, for bosons, equations show notation of the form $2 \Phi_{\iota_{3 L B ; 3 L, 3 B}}^{\iota_{3 C H}}$ and might suggest that each of $L$, conservation of $L, B$, and conservation of $B$ is appropriate. However, discussion related to equation $(76)$ indicates that none of $L$, conservation of $L, B$, and conservation of $B$ is relevant to the relevant boson physics. Each of the first three equations (that is, equations (72), (73), and (74)) correlates with transforming a matter quark into an antimatter simple fermion. Among those equations, the notion of $2 \mathrm{~T}_{-2 ;}^{+1}$, pertains. There are two forms of $2 \mathrm{~T}_{-2,}^{+1}$, , $_{-}$, namely $2 \mathrm{~T}_{-2 ; 0,+2}^{+1}$ and $2 \mathrm{~T}_{-2 ;-3,-1}^{+1}$. The two forms, $2 \mathrm{~T}_{-2 ; 0,+2}^{+1}$ and $2 \mathrm{~T}_{-2 ;-3,-1}^{+1}$, show the same $\iota_{3 L B}$, but do not correlate with the same $L$ or with the same $B$. The fourth equation correlates with transforming a matter quark into a matter fermion. Each one of the second, third, and fourth equations might correlate with the ongoing modeling notion of leptoquarks.

$$
\begin{aligned}
& 1 \mathrm{Q}_{-1 ; 0,+1}^{+2} \rightarrow 1 \mathrm{Q}_{+1 ; 0,-1}^{+1}+2 \mathrm{~T}_{-2 ; 0,+2}^{+1} \\
& 1 \mathrm{Q}_{-1 ; 0,+1}^{+2}+2 \mathrm{~T}_{-2 ;-3,-1}^{+1} \rightarrow 1 \mathrm{C}_{-3 ;-3,0}^{+3} \\
& 1 \mathrm{Q}_{-1 ; 0,+1}^{-1}+2 \mathrm{~T}_{-2 ;-3,-1}^{+1} \rightarrow 1 \mathrm{~N}_{-3 ;-3,0}^{0} \\
& 1 \mathrm{Q}_{-1 ; 0,+1}^{-1}+2 \mathrm{~T}_{+4 ;+3,-1}^{+1} \rightarrow 1 \mathrm{~N}_{+3 ;+3,0}^{0}
\end{aligned}
$$

More generally, equation (76) shows possible charged $2 \mathrm{~T}$ simple bosons that convert simple fermions between matter and antimatter. Equation (77) shows possible $2 \mathrm{~T}$ charged simple bosons that would not convert simple fermions between matter and antimatter. For each of the four possible charged simple bosons, the notation does not show a number $3 L$ and does not show a number $3 B$.

$$
2 \mathrm{~T}_{\mp 2}^{ \pm 1} \text { and } 2 \mathrm{~T}_{ \pm 2}^{ \pm 2}
$$


Table 68: Cases - for zerolike rest mass elementary particles - for modeling regarding refraction and similar phenomena (with the symbol NR denoting the two-element term not physics-relevant)

\begin{tabular}{cccc}
\hline$n_{S A 0}$ & $n_{T A 0}$ & Boson/Fermion & Example \\
\hline-1 & 0 & Boson & Photons \\
-1 & -1 & Fermion & Neutrinos \\
-1 & -1 & Boson & Gluons \\
-1 & 0 & Fermion & NR \\
\hline
\end{tabular}

$$
2 \mathrm{~T}_{ \pm 4 ;}^{ \pm 1} \text { and } 2 \mathrm{~T}_{\mp 4}^{ \pm 2}
$$

This essay de-emphasizes the possibilities that equation (77) shows.

Regarding equation (76), each of the four possibilities, of which one possibility is $2 \mathrm{~T}_{-2}^{+1}$; , correlates with two possible $L$-and- $B$ pairs. We assume that charged $2 \mathrm{~T}$ bosons are ambiguous with respect to each of $L$ and $B$.

Generally, interactions conserve $\iota_{3 L B}$, do not necessarily conserve $L$, and do not necessarily conserve $B$. Non-conservation of $L$ and $B$ correlates with involvement - in the interactions - of $2 \mathrm{~T}^{ \pm}$bosons. One might deploy the six-word phrase rebuttable somewhat conservation of lepton number and the six-word phrase rebuttable somewhat conservation of baryon number. One might deploy, regarding elementary fermions, the seven-word phrase rebuttable always conservation of fermion 3LB number.

\subsubsection{Refraction and similar phenomena}

We explore modeling regarding contexts in which a zerolike rest mass elementary particle interacts with its surroundings. (This exploration correlates with PFS modeling.) Known examples include photons in refractive media. We explore the notion that similar considerations pertain for neutrinos and for gluons.

Table 68 shows four mathematically possible cases. The case of boson and $n_{T A 0}=0$ pertains for G-family forces. The case of fermion and $n_{T A 0}=-1$ pertains for (at least) neutrinos. The case of boson and $n_{T A 0}=-1$ pertains for (at least) gluons. The case of fermion and $n_{T A 0}=0$ is not necessarily physics-relevant. (Proposed modeling does not predict the existence of fermion simple particles for which $\left.n_{T A 0} \neq n_{S A 0}.\right)$ The equation $n_{S A 0}=-1$ correlates with the notion of zerolike rest mass.

We posit that PFS ALG modeling extends to include notions of non-isotropic harmonic oscillators.

Each of equations $(78)$ and $(79)$ offers, based on using the range $-1<n_{S A 0}<0$, a possible basis for modeling regarding a zerolike rest mass elementary particle. (We contrast $-1<n_{S A 0}<0$ with $n_{S A 0}<-1$. Uses of the expression $n_{S A 0}<-1$ pertain for applications related to components of Gfamily forces and not necessarily for other purposes. Regarding applications related to components of G-family forces, see table 62.) In the sense of KMS modeling, $E$ denotes energy, $\vec{P}$ denotes momentum, $\vec{v}$ denotes velocity, $<_{-}>$denotes the expected value of ${ }_{-}, P^{2}=<\vec{P} \cdot \vec{P}>$, and $v^{2}=<\vec{v} \cdot \vec{v}>$. Here, double-entry bookkeeping pertains to models for which at least one of the PFS ALG TA-side set of harmonic oscillators and the PFS ALG SA-side set of harmonic oscillators is not necessarily isotropic.

$$
\begin{gathered}
n_{S A 0}=-c^{2} P^{2} / E^{2} \\
n_{S A 0}=-v^{2} / c^{2}
\end{gathered}
$$

For each of the three physics-relevant cases, each of equations (78) and (79) adds a positive amount to $A_{S A}^{A L G}$. For each of the three cases, we posit that, for each relevant oscillator, $-1 \leq n \leq 0$ pertains.

For the case of boson and $n_{T A 0}=0$, for each relevant TA-side oscillator, $n_{T A}=0$. One cannot satisfy double-entry bookkeeping by adding to $A_{T A}^{A L G}$. Satisfying double-entry bookkeeping correlates with subtracting something positive from at least one of the SA-side oscillators that correlate with $S U(2)$ kinematics symmetries. Proposed modeling correlates this subtracting with aspects of refraction. Ongoing modeling correlates the expression $c / v$ (or, $\left(c^{2} / v^{2}\right)^{1 / 2}$ ) with the two-word term refractive index (or, with the three-word term index of refraction). This case correlates with refraction of light.

For the case of fermion and $n_{T A 0}=-1$, for each relevant SA-side oscillator, $n_{S A}=-1$. One cannot satisfy double-entry bookkeeping by adding to $A_{S A}^{A L G}$. Satisfying double-entry bookkeeping correlates with adding something positive to at least one of the two TA-side oscillators that correlate with $S U(2)$ rebuttable somewhat conservation of generation symmetry or to at least one of the TA-side oscillators that correlate with conservation of energy symmetry. Assuming that conservation of energy pertains, (rebuttable always) conservation of generation does not pertain. This modeling comports with the notion 
Table 69: PFS representation for SA-side aspects regarding 2U erase or paint ground states (with some aspects - for example, TA-side aspects related to conservation of charge - omitted)

\begin{tabular}{|c|c|c|c|}
\hline Ground state & SA0 & SA9 & SA10 \\
\hline $2 \mathrm{U} 0$ & $(-1)^{+}$ & -1 & -1 \\
\hline 2U9 & -1 & $(-1)^{+}$ & -1 \\
\hline 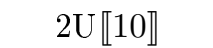 & -1 & -1 & $(-1)^{+}$ \\
\hline
\end{tabular}

of neutrino oscillations. This case correlates with neutrino oscillations. Observations suggest that rates at which neutrinos oscillate vary with the energies of the neutrinos. (See reference [10.) This essay does not pursue the notion that double-entry bookkeeping techniques and an equation such as equation (78) might correlate with a model - for symmetry breaking - that correlates with rates of neutrino oscillations.

For the case of boson and $n_{T A 0}=-1$, equations $80,(81)$ and 82 can pertain. Here, the symbol $n_{S A_{-}}$correlates with an oscillator for which $n_{S A_{-}}=-1$ originally pertained. (Perhaps, compare with discussion, pertaining to refraction, regarding equations (78) and (79).) Here, the notation $a \leftarrow b$ correlates with the three-element phrase $a$ becomes $b$ (or, with the notion that $b$ replaces $a$ ). Here, the notion of $n_{S A}=(-1)^{+}$correlates with concepts such as refraction and with modeling that correlates with non-isotropic harmonic oscillators. Here, discussion is not necessarily as straightforward as is discussion for the other two physics-relevant cases. Discussion related to table 69 pertains regarding gluons.

$$
\begin{gathered}
-1<(-1)^{+}<0 \\
n_{T A 0}=-1 \leftarrow n_{T A 0}=(-1)^{+} \\
n_{S A_{-}}=-1 \leftarrow n_{S A_{-}}=(-1)^{+}
\end{gathered}
$$

\subsubsection{Gluon interactions}

We explore modeling regarding gluons and modeling regarding U-family interactions.

The 2U solutions correlate with gluons. Here, we provide details correlating with PFS ALG modeling.

We denote three relevant PFS oscillators by the symbols SA0, SA9, and SA10. (Perhaps, compare with table 54.) Regarding quark or arc simple fermions, each of oscillators SA0, SA9, and SA10 correlates with a color charge. Relative to an ongoing modeling standard representation for gluons, one of SA9 and SA10 correlates with the color red, the other of SA9 and SA10 correlates with the color blue, and SA0 correlates with the color green.

Table 69 shows aspects regarding three erase or paint ground states.

A gluon correlates with a weighted sum of two or three erase-and-paint pairs. For each pair, the erase part correlates with, in effect, an ability to erase, from the quark or arc simple fermion that absorbs the gluon, a color. The paint part correlates with, in effect, an ability to paint, on to the quark or arc simple fermion that absorbs the gluon, a color. The value $n_{S A}=(-1)^{+}$denotes an ability for a gluon to erase or paint the color charge correlating with the SA_oscillator. Equation (83) shows an ongoing modeling representation for one of the eight gluons. (Out of the eight gluons, this is the only one that involves three erase-and-paint pairs. Each of the other seven gluons involves two erase-and-paint pairs.) Regarding table 69, we make the following correlations. (Alternatively, without loss of generality or results, one might reverse the roles of SA9 and SA10.) The symbol $r$ correlates with painting the color red and with a painting application of $2 \mathrm{U} \llbracket 10 \rrbracket$. The symbol $\bar{r}$ correlates with erasing the color red and with an erasing application of $2 \mathrm{U} \llbracket 10 \rrbracket$. The symbol $b$ correlates with painting the color blue and with a painting application of $2 \mathrm{U} 9$. The symbol $\bar{b}$ correlates with erasing the color blue and with an erasing application of $2 \mathrm{U} 9$. The symbol $g$ correlates with painting the color green and with a painting application of $2 \mathrm{U} 0$. The symbol $\bar{g}$ correlates with erasing the color green and with an erasing application of $2 \mathrm{U} 0$.

$$
(r \bar{r}+b \bar{b}-2 g \bar{g}) /(6)^{1 / 2}
$$

The expression $n_{T A 0}=(-1)^{+}$pertains. (See equation (81).) The expressions $n_{T A 5}=-1$ and $n_{T A 6}=-1$ correlate with a $\kappa_{-1,-1}$ (or, $\left.S U(2)\right)$ symmetry. (Regarding the choice of the oscillator pair TA5-and-TA6, see table 54.) That symmetry might correlate with (at least) rebuttable somewhat conservation of fermion generation. That symmetry might correlate with always conservation of fermion generation. (See discussion - related to the possibility for $2 \mathrm{U}$ strong interaction $\mathrm{CP}$ violation - in table 113.) 


\subsubsection{Numbers of color charges}

Table 58 points to proposed modeling aspects that correlate with exactly three color charges. (Perhaps, see also, table 20.)

The notion of three color charges comports with the notion that $D=3$ pertains regarding TA-side aspects of modeling for fermion elementary particles. (See table 32c.) This discussion might correlate with a notion that three (and no more than three) color charges pertain for each quark and each arc.

\subsubsection{Interactions involving jay bosons}

We note - as perspective - one observational result that might correlate with effects correlating with jay bosons.

Reference [11] reports a discrepancy between the observed energy correlating with one type of finestructure transition in positronium and a prediction based on core ongoing modeling. (Perhaps, see also reference [12.) Equation (84) states a transition frequency. The observed value of transition frequency correlates with the energy that correlates with the transition. Equation 85 correlates with ongoing modeling. The observed energy exceeds the predicted energy. Reference [11] characterizes the transition via the expression $2^{3} S_{1} \rightarrow 2^{3} P_{0}$.

$$
\begin{aligned}
& 18501.02 \pm 0.61 \mathrm{MHz} \\
& 18498.25 \pm 0.08 \mathrm{MHz}
\end{aligned}
$$

We explore the topic of interactions and effects correlating with jay bosons.

Table 70 discusses aspects regarding physics, interactions, and modeling involving jay (or, 2J) simple bosons.

\subsubsection{A series of formulas for lengths, including the Planck length}

We discuss three related formulas that produce lengths. The formulas correlate with aspects pertaining to elementary particles and to other objects.

We suggest a series of formulas for lengths. Equation (86) correlates with the Schwarzschild radius for an object of mass $m$. Equation (87) correlates with the Planck length and does not depend on $m$. Equation (88) includes a factor of $m^{-1}$. When applied to the mass of $2 \mathrm{~W}$ bosons, equation (88) correlates somewhat with the range of the weak interaction. When applied to the mass of a charged pion, equation (88) correlates somewhat with a range for the component, of the strong interaction, that has bases in gluons. (That component binds the two quarks that exist within the pion.) Equation 89 shows the ratio between successive formulas. Equation (90) shows, for the electron, the ratio correlating with equation (89).

$$
\begin{gathered}
R_{4}(m)=\left(G_{N}\right)^{1} m^{1} \hbar^{0} c^{-2} 2^{1} \\
R_{2}(m)=\left(G_{N}\right)^{1 / 2} m^{0} \hbar^{1 / 2} c^{-3 / 2} 2^{0} \\
R_{0}(m)=\left(G_{N}\right)^{0} m^{-1} \hbar^{1} c^{-1} 2^{-1} \\
\left(G_{N}\right)^{-1 / 2} m^{-1} \hbar^{1 / 2} c^{1 / 2} 2^{-1} \\
\left(G_{N}\right)^{-1 / 2}\left(m_{\epsilon}\right)^{-1} \hbar^{1 / 2} c^{1 / 2} 2^{-1} \approx 1.1945 \times 10^{22}
\end{gathered}
$$

\subsubsection{Ranges for interactions that correlate with elementary bosons}

Table 71 discusses ranges that pertain regarding interactions that correlate with elementary bosons. Generally, subsequent rows in table 71 correlate with smaller effective ranges than the effective ranges that correlate with previous rows. Some items in the table correlate with equation (88). 
Table 70: Aspects regarding 2J bosons

(a) Aspects - correlating with observations and modeling - that might correlate with $2 \mathrm{~J}$ bosons

\begin{abstract}
Aspect
- Interactions - between identical fermions - that correlate with ongoing modeling notions of a Pauli exclusion force. (A pair of such identical fermions can be, for example, two hadrons in an atomic nucleus or two elementary particles. In ongoing modeling, the notion of identical can involve rest energy, charge, generation, and - for example, in an atom - spin orientation and orbital state. Aspects such as spin orientation and orbital state correlate with ongoing modeling KMS aspects. Perhaps note table 109. Proposed modeling would include - in the notion of identical - 3LB number. This inclusion would not necessarily add to the list that correlates with ongoing modeling. Proposed modeling would suggest - regarding the notion of identical - including a number that correlates with isomer. This inclusion would add to the list that correlates with ongoing modeling.)

- Forces correlating with some energy levels of positronium atoms. (See discussion related to equation (84).)

- Some interaction vertices that involve an incoming spin-one fermion, an incoming or outgoing $\Sigma G$ for which $\Sigma \geq 4$, and an outgoing spin-one fermion. (See discussion related to equation (124). For this example, a 2J boson absorbs, in effect, one unit of spin that correlates originally with an incoming fermion. The unit correlates with $\hbar$.)

- Some interaction vertices that involve no fermions. (See discussion related to equation 163 . For this example, a 2J boson might correlate with, in effect, two units of spin that correlates with an outgoing component of a graviton. The unit correlates with $\hbar$.)
\end{abstract}

(b) Suggested aspects regarding $2 \mathrm{~J}$ bosons

\begin{tabular}{|c|}
\hline Aspect \\
\hline 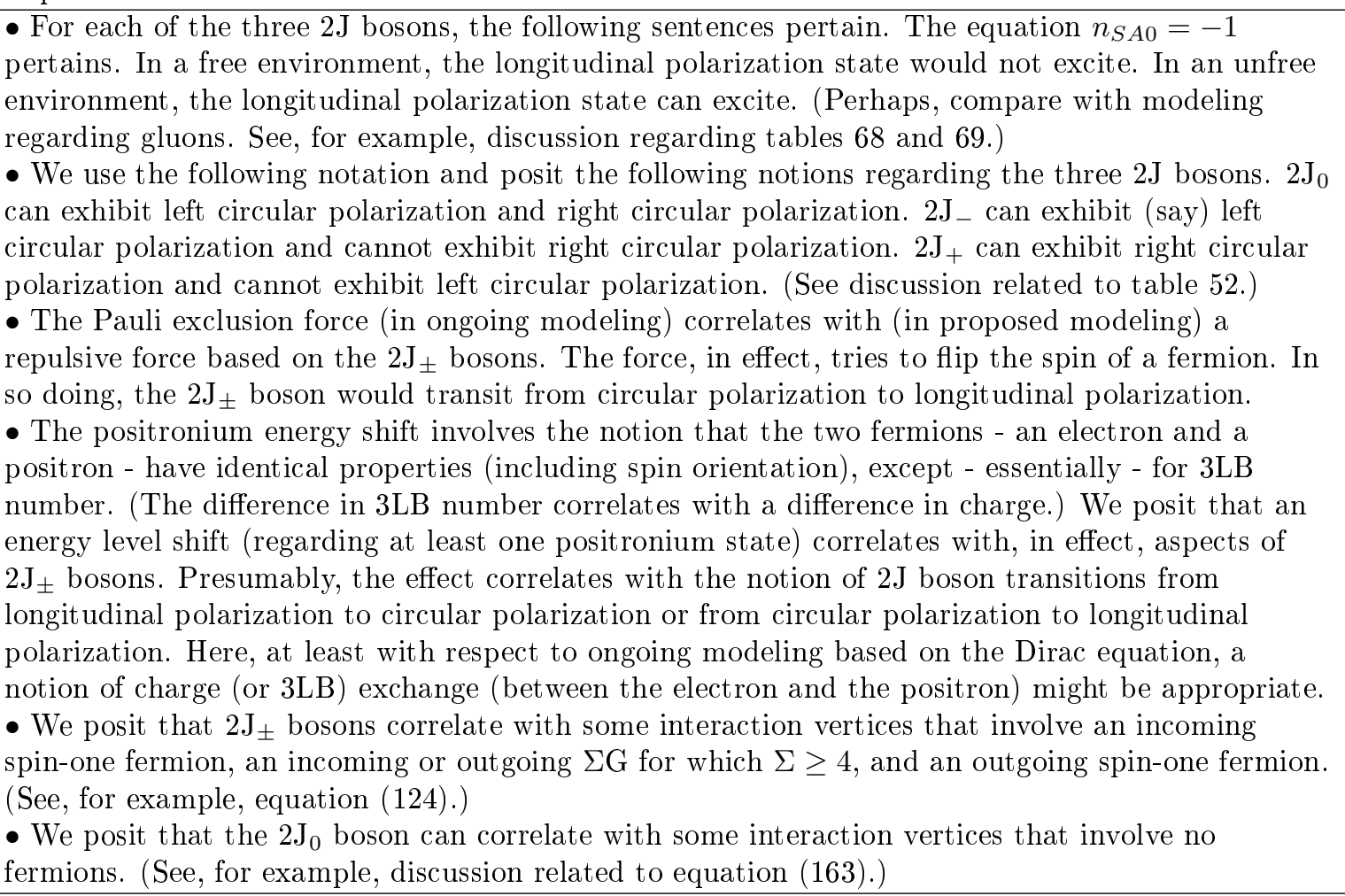 \\
\hline
\end{tabular}


Table 71: Ranges for interactions that correlate with elementary bosons

\begin{tabular}{|c|c|c|c|}
\hline$n_{T A 0}$ & $n_{S A 0}$ & Subfamilies & $\begin{array}{l}\text { Discussion regarding effective ranges regarding interactions mediated } \\
\text { between elementary particles and hadron-like particles }\end{array}$ \\
\hline 0 & -1 & $\Sigma \mathrm{G}, \Sigma \geq 2$ & $\begin{array}{l}\text { - The photon, graviton, and so forth have effectively unbounded } \\
\text { ranges. }\end{array}$ \\
\hline 0 & 0 & $0 \mathrm{P}$ & $\begin{array}{l}\text { - The pie has a range that correlates with the distance, within an } \\
\text { atomic nucleus, between neighboring hadrons. }\end{array}$ \\
\hline-1 & -1 & $2 \mathrm{U}$ & - Gluons have a range of the size of a hadron-like particle. \\
\hline 0 & 0 & $0 \mathrm{H}, 2 \mathrm{~W}$ & $\begin{array}{l}\text { - The range of the weak interaction is about } 10^{-18} \text { meters, in the } \\
\text { sense that } 2 \mathrm{G} \text { (if relevant) has more effect (than the weak } \\
\text { interaction) at distances greater than about } 10^{-18} \text { meters. }\end{array}$ \\
\hline 0 & 0 & $2 \mathrm{~T}$ & $\begin{array}{l}\text { - Proposed modeling suggests that tweak masses are larger than the } \\
\text { mass of the Higgs boson and that the range for tweak bosons is less } \\
\text { than the range for the weak interaction. }\end{array}$ \\
\hline-1 & -1 & $0 \mathrm{I}$ & $\begin{array}{l}\text { - The 0I particle may lose its effectiveness (at least, relative to effects } \\
\text { of other relevant interactions) regarding interactions when densities } \\
\text { are somewhat less than densities pertaining to the end of the } \\
\text { inflationary epoch. }\end{array}$ \\
\hline-1 & -1 & 2.J & $\begin{array}{l}\text { - Regarding interactions with fermions, jay particles have effective } \\
\text { ranges that are similar to the effective range of the ongoing modeling } \\
\text { Pauli exclusion force. The ranges related to jay bosons might vary } \\
\text { based on whether modeling pertains, for example, to interactions } \\
\text { between elementary fermions or to interactions between fermion } \\
\text { hadron-like particles. } \\
\text { - Regarding interactions with elementary bosons, jay particles might } \\
\text { lose their effectiveness (at least, relative to effects of other relevant } \\
\text { interactions) regarding interactions when densities are less than } \\
\text { densities pertaining to the start of the inflationary epoch. }\end{array}$ \\
\hline
\end{tabular}

Table 72: Correlations between $0 \mathrm{G}$ and $0 \mathrm{U}$ solutions and simple bosons

\begin{tabular}{ccccl}
\hline $\begin{array}{c}\text { Direct basis } \\
\text { (solutions) }\end{array}$ & $\begin{array}{c}\text { Other } \\
\text { basis }\end{array}$ & Subfamily & Bosons & Discussion related to ... \\
\hline $0 \mathrm{G} 2468$ & - & $0 \mathrm{H}$ & Higgs & Table 73 \\
- & $0 \mathrm{H}$ & $0 \mathrm{I}$ & Aye & Table 53 \\
$0 \mathrm{G} 246$ and $0 \mathrm{G} 268$ & - & $2 \mathrm{~W}$ & Z and W & Table 73 \\
$0 \mathrm{G} \Gamma_{\lambda_{\max }=14}$ & - & $2 \mathrm{~T}$ & Tweaks & Equation 91 \\
$0 \mathrm{G} \emptyset$ & - & $2 \mathrm{~J}$ & Jays & Table 74 \\
$0 \mathrm{U}$ & - & $0 \mathrm{P}$ & Pie & Table 55 \\
\hline
\end{tabular}

\subsubsection{Correlations between simple particles and G-family and U-family ALG solutions}

Work elsewhere discusses the notion that PDE equations and solutions correlate with all the elementary particles that proposed modeling suggests. (See table 57.)

Table 72 discusses, from a perspective of ALG modeling, the notion that 0G and 0U solutions might correlate with all simple bosons. The leftmost column points to G-family solutions $\Sigma \mathrm{G}$ that correlate with $\Sigma=0$ and to one possible U-family solution. For each row, other than the row pertaining to the OI subfamily, proposed modeling suggests the possibility that subfamilies and bosons correlate - from a perspective of some modeling - with the solutions that the table list for that row. Table 72 suggests a possible (indirect) correlation (via modeling for the Higgs boson) regarding the aye boson. For each row, the rightmost column points to aspects of this essay that discuss the suggested correlations.

We discuss the Higgs boson and the weak interaction bosons.

Table 73 posits correlations between all $\mathrm{W}$-family and $\mathrm{H}$-family bosons and all G-family $\Sigma=0$ solutions - other than $0 \mathrm{G} \emptyset$ - that correlate with table 62 . The symbol $j_{\lambda \in \Gamma}$ denotes the number of elements in the $\Gamma$ in $\Sigma$ G $\Gamma$. Elsewhere, we correlate $j_{\lambda \in \Gamma}$ with mass. (See discussion regarding table 76 .)

Each of the $\Sigma=0$ items that table 73 lists has a TA-side symmetry of none or $S U(3)$. (See table 62 .) We discuss the aye boson.

We correlate the mass of the aye (or, 0I) boson with the result $D+2 \nu^{\prime \prime}=0$ that pertains for the row 
Table 73: Possible correlations between 0G solutions and all W-family and H-family bosons (with $j_{\lambda \in \Gamma}$ denoting the number of elements in the $\Gamma$ in $\Sigma \mathrm{G} \Gamma$ )

\begin{tabular}{cccc}
\hline Solutions & Bosons & Subfamily & $j_{\lambda \in \Gamma}$ \\
\hline 0G246 and 0G268 & Z and W & $2 \mathrm{~W}$ & 3 \\
0G2468 & $\mathrm{H}^{0}$ (or, Higgs) & 0H & 4 \\
\hline
\end{tabular}

Table 74: Possible direct correlation between 0G solutions and jay bosons (with $j_{\lambda \in \Gamma}$ denoting the number of elements in the $\Gamma$ in $\Sigma \mathrm{G} \Gamma$ )

\begin{tabular}{cccc}
\hline Solution & Bosons & Subfamily & $j_{\lambda \in \Gamma}$ \\
\hline $0 \mathrm{G} \emptyset$ & Three 2J (or, jay) bosons & 2J & 0 \\
\hline
\end{tabular}

- in table $32 \mathrm{e}$ - that pertains for $S^{\prime \prime}=1$ and $\Omega^{\prime \prime}=1$.

Proposed modeling correlates the aye boson with the Higgs boson. (See discussion related to table 53.) Thereby, proposed modeling suggests - per table 73 - a correlation between the aye boson and G-family solutions.

We discuss the tweak bosons.

Discussion immediately above involves (at least indirectly) $\Omega^{\prime \prime} \leq 0$ rows in table $32 \mathrm{e}$ that correlate with values of $S^{\prime \prime}$ of zero, three, and four.

The next opportunity for $0 \mathrm{G}$ solutions correlates with the range $2 \leq \lambda \leq \llbracket 14 \rrbracket$. For that range, there are four solutions that correlate with 0G. Equation (91) shows the solutions. Here, we do not put brackets around values of $\lambda$ that exceed eight. Equation (92) shows the same four solutions, but with a different grouping. For each of equation (91) and equation (92), the first item (and, hence, the first two solutions) correlates with the expression $0 \pm 0$.

$$
(14-10-6+2) \pm(12-8-4) ; \quad 14-12-10+8+6-4-2 ; 14-12+10-8-6+4-2
$$

$$
(14-12-10+8) \pm(6-4-2) ; 14+12-10-8-6-4+2 ; 14-12+10-8-6+4-2
$$

Proposed modeling suggests that $2 \mathrm{~T}^{0^{\prime \prime}}$ and $2 \mathrm{~T}^{0^{\prime}}$ correlate with two solutions that equation (91) shows. The other two solutions that the equation shows would correlate with $2 \mathrm{~T}^{2}$ and $2 \mathrm{~T}^{1}$. We use these results to estimate masses for $2 \mathrm{~T}$ simple bosons. (See discussion related to equations $(98)$ and $(99)$.)

We discuss the jay bosons.

Table 74 notes a possible direct correlation between 0G solutions and jay bosons. (See, also, discussion related to equation (95).) We correlate the mass of jay bosons with the result $D+2 \nu^{\prime \prime}=1$ that pertains for the row - in table $32 \mathrm{e}$ - that pertains for $S^{\prime \prime}=0$ and $\Omega^{\prime \prime}=0$.

We discuss the pie boson.

Table 55 suggests correlating the 0P boson with the U-family and not necessarily with the G-family. This essay does not explore mathematics that might correlate modeling for the $0 \mathrm{P}$ boson with a notion of a $0 \mathrm{U}$ solution.

We discuss the possibility of correlations between $0 \mathrm{G}$ solutions and simple fermions.

Table 75 suggests correlations between $2 \mathrm{~W}$ solutions (and hence, via table 72 , 0G solutions) and solutions that correlate with all simple fermions. Table $75 \mathrm{~b}$ restates - for $\mathrm{W}$ bosons - table 42 . Table $75 \mathrm{c}$ reflects aspects of table $75 \mathrm{~b}$, based on notions that the next three sentences mention. The TA0 result correlates with transforming 0 to $@_{0}$ (which is appropriate for fermions). The SA1-and-SA2 result $\pi_{0} @_{0} @_{-1}$ correlates with transforming 0 to $@_{0}$ (which is appropriate for fermions) and transforming one $@_{0}$ to $@_{-1}$ (which is also appropriate for fermions). The TA5-and-TA6 result correlates with applying double-entry bookkeeping and with transforming $\kappa_{0,0}$ to $\kappa_{0,-1}$ (for which the notation of [blank] is appropriate). From here, one presumably can mirror steps - that this essay shows elsewhere - that lead to representations for individual simple $1 \mathrm{C}$ fermions, individual simple $1 \mathrm{~N}$ fermions, individual simple 1Q fermions, and individual simple $1 \mathrm{R}$ fermions. (See discussions related to table 45 , table 46 , and table 50.)

\subsection{Predictions and correlations regarding properties of elementary particles}

We explore masses and other properties of elementary particles. 
Table 75: Indirect correlations between 0G solutions and simple fermions

(a) Bases

\begin{tabular}{ccccl}
\hline $\begin{array}{c}\text { Direct basis } \\
\text { (solutions) }\end{array}$ & $\begin{array}{c}\text { Other } \\
\text { basis }\end{array}$ & Subfamily & Fermions & Discussion related to $\ldots$ \\
\hline- & $2 \mathrm{~W}$ & $1 \mathrm{C}$ & Charged leptons & Table $75 \mathrm{~b}$ and table $75 \mathrm{c}$ \\
- & $1 \mathrm{C}$ & $1 \mathrm{~N}$ & Neutrinos & Table 46 \\
- & $1 \mathrm{C}$ & $1 \mathrm{Q}$ & Quarks & Table 50 \\
- & $1 \mathrm{~N}$ & $1 \mathrm{R}$ & Arcs & Table 50 \\
\hline
\end{tabular}

(b) A PFS-centric representation for the ground state for W bosons

\begin{tabular}{cccccccccc}
\hline Side & 0 & 1,2 & 3,4 & 5,6 & 7,8 & 9,10 & 11,12 & 13,14 & 15,16 \\
\hline TA & 0 & & & $\kappa_{0,0}$ & $\pi_{@_{0}, @_{-1}}$ & & & & \\
SA & $@_{0}$ & $\pi_{0, @_{0}}$ & & & & & & & \\
\hline
\end{tabular}

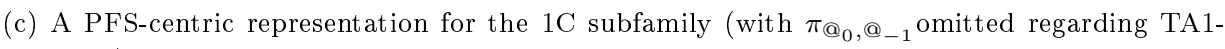
and-TA2)

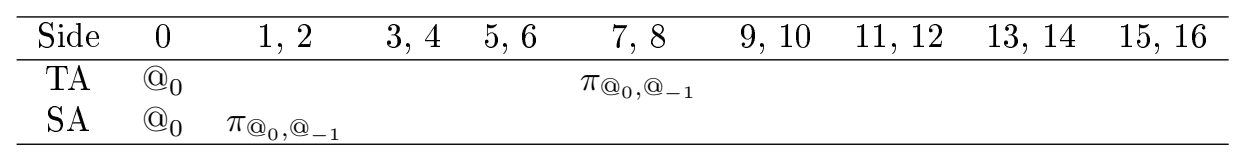

Table 76: Rest energies for known non-zero-mass simple bosons

\begin{tabular}{cccccccc}
\hline \multirow{2}{*}{$\Phi$} & \multirow{2}{*}{$S$} & \multirow{2}{*}{ Symbol } & \multirow{2}{*}{ Name } & $\begin{array}{c}\text { Experimental } \\
m c^{2}(\mathrm{GeV})\end{array}$ & \multicolumn{2}{c}{ Calculated } & Difference \\
\cline { 5 - 7 } & & & $\mathrm{N}$ & $m c^{2}(\mathrm{GeV})$ & (standard deviations) \\
\hline $\mathrm{W}$ & 1 & $2 \mathrm{~W} 1,2 \mathrm{~W} 2$ & $\mathrm{~W}$ & $80.379 \pm 0.012$ & 7 & 80.420 & $\approx 3.4$ \\
$\mathrm{~W}$ & 1 & $2 \mathrm{~W} 0$ & $\mathrm{Z}$ & $91.1876 \pm 0.0021$ & 9 & 91.1876 & - \\
$\mathrm{H}$ & 0 & $0 \mathrm{H} 0$ & $\mathrm{H}^{0}$ & $125.18 \pm 0.16$ & 17 & 125.33 & $\approx 1.0$ \\
\hline
\end{tabular}

\subsubsection{The masses of the $W, Z$, and Higgs bosons}

We explore relationships between masses of the $2 \mathrm{~W}$ (or, $\mathrm{W}$ and $\mathrm{Z}$ ) and $0 \mathrm{H}$ (or, Higgs) bosons.

Table 76 shows, in the column for which the label includes the word experimental, rest energies for the known non-zero-mass simple bosons. (See reference [13.) Notation such as $2 \mathrm{~W} 1$ and $0 \mathrm{H} 0$ extends the notion of $\Gamma$ - as pertaining to oscillators relevant in ALG models for G-family solutions - to notions of $\Gamma$ for ALG models relevant to elementary particle families other than the $G$ family. The most accurately known of the three masses is the mass of the $\mathrm{Z}$ boson. (Rest energy equals mass times $c^{2}$.) The column for which the label includes the word calculated shows results based on equation (93) and on assuming that nine correlates with the square of the mass of the $\mathrm{Z}$ boson. Equation (94) shows the size of one unit. The related mass is $\approx 30.396 \mathrm{GeV} / c^{2}$. In table 76 , the column for which the label includes the word difference shows the number of standard deviations (regarding the experimental results) by which the calculated mass differs from the nominal experimental mass.

$$
\begin{gathered}
\left(m_{\mathrm{H}^{0}}\right)^{2}:\left(m_{\mathrm{Z}}\right)^{2}:\left(m_{\mathrm{W}}\right)^{2}:: 17: 9: 7 \\
\approx 9.239 \times 10^{2}\left(\mathrm{GeV} / c^{2}\right)^{2}:: 1
\end{gathered}
$$

We discuss approximate ratios for the squares of masses of the Higgs, Z, and W bosons.

Based on the ratios (of squares of masses) that equation (93) shows, the possibly least accurately suggested mass is that of the $\mathrm{W}$ boson. Equation (93) correlates with a number that is within four standard deviations of the nominal mass of the $\mathrm{W}$ boson. (See table 76 .)

Proposed modeling correlates the numbers in equation (93) with, respectively, the expressions $17=17$, $9=10-1-0$, and $7=10-1-2$. Each of zero, one, two, 10, and 17 correlates with the value of $D+2 \nu^{\prime \prime}$ for a PDE solution for which $D^{\prime \prime}=2$. (See table 32e.)

The following correlations pertain regarding relative squares of masses. (See table 73 and table 32e.) For each of the $\mathrm{W}, \mathrm{Z}$, and $\mathrm{OH}$ bosons, one positive term pertains. That term correlates with the value of $D+2 \nu^{\prime \prime}$ for which $\sigma^{\prime \prime}=-1$ and $S^{\prime \prime}=j_{\lambda \in \Gamma}$ pertain. (See table 73.) For the $\mathrm{W}$ and $\mathrm{Z}$ bosons, a negative term - minus one - pertains. That term correlates with the negative of the value of $D+2 \nu^{\prime \prime}$ 
for which $\sigma^{\prime \prime}=-1$ and $S^{\prime \prime}=0$ pertain. That term might correlate with spin-one. (See discussion related to equation (136). See also discussion - regarding the mass of the 2J bosons - related to equation (95).) For the $\mathrm{W}$ boson, another negative term - minus 2 - also pertains. That term correlates with the negative of the value of $D+2 \nu^{\prime \prime}$ for which $\sigma^{\prime \prime}=-1$ and $S^{\prime \prime}=1$ pertain. That term might correlate with the magnitude of a nominal magnetic dipole moment (or that term might correlate with a charge of magnitude equal to the magnitude of the charge of the electron). (Perhaps, see discussion related to equation (136).)

To the extent that $m_{\mathrm{W}}$ does not exactly comport with equation (93), proposed modeling suggests the possibility that an anomalous moment pertains. The $\mathrm{W}$ boson has non-zero charge, non-zero nominal magnetic dipole moment, and non-zero mass. We suggest that the anomalous moment might correlate mostly with the 6G24 solution. (Compare with discussion related to equation (196).) The contribution of minus two (compared to the $\mathrm{Z}$ boson) - that equation (93) implies - might correlate with each of $2 \mathrm{G} 24$ and nominal magnetic dipole moment.

\subsubsection{The masses of the aye boson and jay bosons}

We explore modeling for the mass of the jay (or, 2J) bosons.

For the jay bosons, correlation with the $0 \mathrm{G} \emptyset$ solution seems to be appropriate. The $0 \mathrm{G} \emptyset$ solution correlates with $j_{\lambda \in \Gamma}=0$. (Compare with discussion related to table 73.) The result $j_{\lambda \in \Gamma}=0$ might correlate with $S^{\prime \prime}=0$. (See discussion related to table 76.) The result $S^{\prime \prime}=0$ correlates with a relative square of mass of one. (See the column - in table $32 \mathrm{e}$ - labeled $D+2 \nu^{\prime \prime}$.) Paralleling results for the $\mathrm{Z}$ and $\mathrm{W}$ bosons, the square of the masses of the jay bosons would be proportional to plus one minus one, which is zero. (The term minus one correlates with the notion of subtracting one for elementary bosons that have spins of one.) Equation (95) extends equation (93).

$$
\left(m_{\mathrm{H}^{0}}\right)^{2}:\left(m_{\mathrm{Z}}\right)^{2}:\left(m_{\mathrm{W}}\right)^{2}:\left(m_{2 \mathrm{~J}}\right)^{2}:: 17: 9: 7: 0
$$

We explore modeling for the mass of the aye (or, 0I) boson.

We suggest that the aye boson correlates with the solution for which $\sigma^{\prime \prime}=+1$ and $S^{\prime \prime}=1$. (See table 32e.) The result $D+2 \nu^{\prime \prime}=0$ pertains and correlates with a zero square of mass.

\subsubsection{Possible masses of the tweak bosons}

We explore possibilities regarding masses of T-family bosons.

We explore using patterns that have bases in G-family solutions and in aspects of table 32e.

Table 77 points to a possible mass for zero-charge $2 \mathrm{~T}$ bosons. Table $77 \mathrm{a}$ summarizes information pertaining to some simple bosons. The $D+2 v^{\prime \prime}$ column shows information - from table $32 \mathrm{e}-$ that correlates with the relevant positive contribution to the sums that correlate with the squares of the masses of the simple bosons. The $\Omega^{\prime \prime}$ and $S^{\prime \prime}$ columns show other information from table 32e. For other than the 0I boson, the $j_{\lambda \in \Gamma}$ shows the number of oscillator pairs relevant to solutions $\Sigma$ G $\Gamma$ for which $\Sigma=0$. (See, for example, discussion related to table 76.) The next column shows the relevant negative contribution that correlates with spin. (See, for example, discussion related to table 76.) The last column sums the $D+2 v^{\prime \prime}$ integer and the spin-related integer. (See table 76 for a use of the symbol N.) Table $77 \mathrm{~b}$ extrapolates from table $77 \mathrm{a}$. The equation $S^{\prime \prime}=7$ provides the first possibility (beyond the limit $\lambda \leq 8)$ to have G-family-like solutions for which $\Sigma=0$. (See discussion related to equation (91).) The equation $S^{\prime \prime}=7$ would correlate with allowed values of $\lambda$ of two, four, six, eight, 10, 12, and 14. For $S^{\prime \prime}=7, D+2 \nu^{\prime \prime}=50$.

The charges for the $\mathrm{T}^{ \pm 1}$ and $\mathrm{T}^{ \pm 2}$ bosons are between the charges for the $\mathrm{Z}^{0}$ and $\mathrm{W}^{ \pm 3}$ bosons. We assume that the relevant charge-related (or magnetic-moment-related) contributions to the total values of $\mathrm{N}$ are negative numbers that fall in the range of minus two to zero. Equations (96) and (97) pertain. (Here, $\mathrm{T}^{0}$ denotes each of $\mathrm{T}^{0^{\prime \prime}}$ and $\mathrm{T}^{0^{\prime}}$. Here, $\mathrm{T}^{ \pm}$denotes each of $\mathrm{T}^{ \pm 2}$ and $\mathrm{T}^{ \pm 1}$.) Based on data from reference [13] regarding the Higgs boson, the rest energies of the T-family bosons would comport with equations (98) and (99).

$$
\begin{gathered}
\left(m_{\mathrm{T}^{0}}\right)^{2} /\left(m_{\mathrm{H}^{0}}\right)^{2}=49 / 17 \\
47 / 17 \leq\left(m_{\mathrm{T}^{ \pm}}\right)^{2} /\left(m_{\mathrm{H}^{0}}\right)^{2} \leq 49 / 17 \\
\left(m_{\mathrm{T}^{0}}\right) c^{2} \approx 212.5 \mathrm{GeV}
\end{gathered}
$$


Table 77: Patterns regarding the squares of masses of some simple bosons

(a) Results for the 0I, 2J, 2W, and $0 \mathrm{H}$ bosons

\begin{tabular}{ccccccc}
\hline Simple bosons & $D+2 v^{\prime \prime}$ & $\Omega^{\prime \prime}$ & $S^{\prime \prime}$ & $j_{\lambda \in \Gamma}$ & Spin-related & N for zero-charge simple bosons \\
\hline 0I & 0 & 1 & 1 & $(-1)$ & 0 & 0 \\
$2 \mathrm{~J}$ & 1 & 0 & 0 & 0 & -1 & 0 \\
$2 \mathrm{~W}$ & 10 & -9 & 3 & 3 & -1 & 9 \\
$0 \mathrm{H}$ & 17 & -16 & 4 & 4 & 0 & 17 \\
\hline
\end{tabular}

(b) Extrapolation regarding $2 \mathrm{~T}$ bosons

\begin{tabular}{ccccccc}
\hline Simple bosons & $D+2 v^{\prime \prime}$ & $\Omega^{\prime \prime}$ & $S^{\prime \prime}$ & $j_{\lambda \in \Gamma}$ & Spin-related & N for zero-charge simple bosons \\
\hline $2 \mathrm{~T}$ & 50 & -49 & 7 & 7 & -1 & 49 \\
\hline
\end{tabular}

$$
208 \mathrm{GeV} \lesssim\left(m_{\mathrm{T}^{ \pm}}\right) c^{2} \lesssim 213 \mathrm{GeV}
$$

Proposed modeling suggests that equations (98) and (99) correlate with the lowest possible rest energies for tweaks. (Possibly, for example, the mass range correlates with, for example, $S^{\prime \prime}=7, S^{\prime \prime}=8$, $S^{\prime \prime}=11$, or $S^{\prime \prime}=12$.)

Detecting $2 \mathrm{~T}^{ \pm}$particles might require an energy of at least twice the rest energy of a $2 \mathrm{~T}^{ \pm}$.

\subsubsection{A prediction for the tauon mass}

Equation 100 shows an experimental result for the tauon mass, $m_{\tau}$. (See reference [14].)

$$
m_{\tau, \text { experimental }} \approx(1776.86 \pm 0.12) \mathrm{MeV} / c^{2}
$$

Equation (101) defines the symbol $\beta^{\prime}$. Equation 102 defines $\beta$. Here, $m$ denotes mass, $\epsilon$ denotes electron, $q$ denotes charge, $\varepsilon_{0}$ denotes the vacuum permittivity, and $G_{N}$ denotes the gravitational constant. Equation (103) possibly pertains. Equation (103) predicts a tauon mass, which equation (104) shows, with a standard deviation of less than one eighth of the standard deviation correlating with the experimental result. (For relevant data, see reference [14].) Equation (105) shows an approximate value of $\beta$ that we calculate, using data that reference [14] shows, via equation (102).) Elsewhere, we correlate the numbers four and three in the left-hand side of equation (102) with a notion of channels. (See discussion related to equation 130 and discussion related to equation (134).)

$$
\begin{gathered}
\beta^{\prime}=m_{\tau} / m_{\epsilon} \\
(4 / 3) \times \beta^{12}=\left(\left(q_{\epsilon}\right)^{2} /\left(4 \pi \varepsilon_{0}\right)\right) /\left(G_{N}\left(m_{\epsilon}\right)^{2}\right) \\
\beta^{\prime}=\beta \\
m_{\tau, \text { calculated }} \approx(1776.8400 \pm 0.0115) \mathrm{MeV} / c^{2} \\
\beta \approx 3477.1891 \pm 0.0226
\end{gathered}
$$

To the extent that equation (103) correlates with nature, a more accurate experimental determination of $G_{N}$ or $m_{\tau}$ could predict a more accurate (than experimental results) value for, respectively, $m_{\tau}$ or $G_{N}$.

Proposed modeling does not, as yet, suggest a relationship - perhaps similar to equation (102) regarding the ratio $m_{\mu} / m_{\epsilon}$. Here, $\mu$ denotes muon. (See discussion related to equations (119) and (120).)

\subsubsection{The masses of quarks and charged leptons}

Table 78 supports the concept that a formula can link the masses of the six quarks and three charged leptons. The table shows values of $\log _{10}\left(m / m_{\epsilon}\right)$. The symbol $m$ denotes an approximate mass. The symbol $m_{\epsilon}$ denotes the mass of the electron. (Discussion regarding table 79 points to the source for relevant data.) For each column, the value increases as one moves downward. For each row that shows more than one value, the value increases as one moves rightward. For each quark column, the charge of 
Table 78: Non-zero charge simple fermions and values of $\log _{10}\left(m / m_{\epsilon}\right)$

\begin{tabular}{ccc}
\hline Charged lepton and value & Quark and value & Quark and value \\
\hline electron: 0.0 & up: 0.6 & down: 1.0 \\
& strange: 2.3 & charm: 3.4 \\
muon: 2.3 & bottom: 3.9 & top: 4.5 \\
tauon: 3.6 & & \\
\hline
\end{tabular}

Table 79: Approximate rest energies (in $\mathrm{MeV}$ ) for quarks and charged leptons

\begin{tabular}{|c|c|c|c|c|c|}
\hline$M^{\prime \prime}$ & Legend & $\begin{array}{c}M^{\prime} \\
\text { Charge }\end{array}$ & $\begin{array}{c}3 \\
-1 \cdot\left|q_{\epsilon}\right|\end{array}$ & $\begin{array}{c}2 \\
+(2 / 3) \cdot\left|q_{\epsilon}\right|\end{array}$ & $\begin{array}{c}1 \\
-(1 / 3) \cdot\left|q_{\epsilon}\right|\end{array}$ \\
\hline 0 & name & & electron & up & down \\
\hline 0 & data & & $(0.511$ to 0.511$) \times 10^{0}$ & $(1.8$ to 2.7$) \times 10^{0}$ & $(4.4$ to 5.2$) \times 10^{0}$ \\
\hline 0 & calculation & & $m_{\epsilon} c^{2} \approx 0.511 \times 10^{0}$ & $m_{u} c^{2} \approx 2.2 \times 10^{0}$ & $m_{d} c^{2} \approx 4.8 \times 10^{0}$ \\
\hline 1 & name & & & charm & strange \\
\hline 1 & data & & & $(1.24$ to 1.30$) \times 10^{3}$ & $(0.92$ to 1.04$) \times 10^{2}$ \\
\hline 1 & calculation & & & $m_{c} c^{2} \approx 1.263 \times 10^{3}$ & $m_{s} c^{2} \approx 0.938 \times 10^{2}$ \\
\hline 2 & name & & muon & top & bottom \\
\hline 2 & data & & $(1.06$ to 1.06$) \times 10^{2}$ & $(1.56$ to 1.74$) \times 10^{5}$ & $(4.15$ to 4.22$) \times 10^{3}$ \\
\hline 2 & calculation & & $m_{\mu} c^{2} \approx 1.06 \times 10^{2}$ & $m_{t} c^{2} \approx 1.72 \times 10^{5}$ & $m_{b} c^{2} \approx 4.18 \times 10^{3}$ \\
\hline 3 & name & & tauon & & \\
\hline 3 & data & & $(1.777$ to 1.777$) \times 10^{3}$ & & \\
\hline 3 & calculation & & $m_{\tau} c^{2} \approx 1.777 \times 10^{3}$ & & \\
\hline
\end{tabular}

the quark in the second row is the same as the charge of the quark in the third row and is not the same as the charge of the quark in the first row.

Table 79 shows, regarding the rest energies of quarks and charged leptons, data that people report and numbers that we calculate via equation (108). Below, we discuss the table and the data before we discuss the equation and the calculations. Equation (108) results from fitting data. This essay does not show modeling that would generate equation (108).

Regarding the placement of quarks, some placements in table 79 differ from the respective placements in table 78. In table 79, the variable $M^{\prime}$ and the columns related to quarks reflect the concept that some aspects regarding mass correlate with charge. In table 79 , for each quark column, each of the charge of the quark in the second row and the charge of the quark in the third row is the same as the charge of the quark in the first row.

The data in table 79 reflect information from reference [13].) For each particle other than the top quark, reference [13] provides one estimate. For the top quark, reference [13] provides three estimates. For each quark, table 79 shows a data range that runs from one standard deviation below the minimum nominal value that reference [13] shows to one standard deviation above the maximum nominal value that reference [13] shows. Each standard deviation correlates with the reported standard deviation that correlates with the nominal value. For charged leptons (that is, for $M^{\prime}=3$ ), the table does not completely specify accuracy regarding ranges.

The following concepts pertain regarding developing equation (108). Equation (106) produces a meaningful value for $m(3,1)$. (No elementary particle correlates with $M^{\prime \prime}=3$ and $M^{\prime}=1$.) For each $0 \leq M^{\prime \prime} \leq 3$, equation (107) produces a meaningful value of $m\left(M^{\prime \prime}, 3 / 2\right)$. (No elementary particle correlates with $M^{\prime}=3 / 2$. Aspects of equations (108), (112), and (113) correlate with the concept that $m\left(M^{\prime \prime}, 3 / 2\right)$ values have meaning.) Within each row for which $M^{\prime \prime} \neq 3$, the fine-structure constant plays a role regarding linking the masses that pertain for that row. (Aspects of equation (108) comport with this role.)

$$
\begin{gathered}
m(3,1) m(3,2)=m(3,0) m(3,3) \\
\left(m\left(M^{\prime \prime}, 3 / 2\right)\right)^{2}=m\left(M^{\prime \prime}, 2\right) m\left(M^{\prime \prime}, 1\right)
\end{gathered}
$$


Table 80: Ranges of $d^{\prime}\left(M^{\prime \prime}\right)$ that fit the data ranges that table 79 shows for quark masses

\begin{tabular}{cccc}
\hline Symbol & $\begin{array}{c}\text { Minimum } \\
\text { (approximate) }\end{array}$ & $\begin{array}{c}\text { Nominal } \\
\text { (table 79) }\end{array}$ & $\begin{array}{c}\text { Maximum } \\
\text { (approximate) }\end{array}$ \\
\hline$d^{\prime}(0)$ & 0.251 & 0.318 & 0.386 \\
$d^{\prime}(1)$ & -1.072 & -1.057 & -1.042 \\
$d^{\prime}(2)$ & -1.5158 & -1.5091 & -1.5024 \\
\hline
\end{tabular}

The following concepts pertain regarding developing and using equation (108). We use equation (102) to calculate $\beta$. Equation (108) calculates the same value of $m_{\tau}$ that equation (104) calculates.

Equation (108) shows a formula that approximately fits the masses of the six quarks and three charged leptons. The formula includes two integer variables and seven parameters. One integer variable, $M^{\prime \prime}$, correlates somewhat with generation. For the electron and each of the six quarks, the generation equals $M^{\prime \prime}+1$. For each of the muon and the tauon, the generation equals $M^{\prime \prime}$. The other integer variable, $M^{\prime}$, correlates with magnitude of charge. The seven parameters can be $m_{\epsilon}, m_{\mu}$ (or, the mass of a muon), $\beta$, $\alpha, d^{\prime}(0), d^{\prime}(1)$, and $d^{\prime}(2)$. The symbol $\alpha$ denotes the fine-structure constant. (See equation (109).) Here, $d^{\prime}(k)$ pertains regarding generation- $(k+1)$ quarks. For each generation, the number $d^{\prime}(k)$ correlates with the extent to which the two relevant quark masses do not equal the geometric mean of the two quark masses. (See equation (107).) Regarding charged leptons, $M^{\prime}=3$, the term $1-\delta\left(M^{\prime}, 3\right)$ is zero, and the factor - in equation (108) - that includes the fine-structure constant is one. (See equation (112).)

$$
\begin{aligned}
& m\left(M^{\prime \prime}, M^{\prime}\right)=m_{\epsilon} \times\left(\beta^{1 / 3}\right)^{M^{\prime \prime}+\left(j_{M^{\prime \prime}}^{\prime \prime}\right) d^{\prime \prime}} \times\left(\alpha^{-1 / 4}\right)^{\left(1-\delta\left(M^{\prime}, 3\right)\right) \cdot\left((3 / 2) \cdot\left(1+M^{\prime \prime}\right)+\left(j_{M^{\prime}}^{\prime}\right) d^{\prime}\left(M^{\prime \prime}\right)\right)} \\
& \alpha=\left(\left(q_{\epsilon}\right)^{2} /\left(4 \pi \varepsilon_{0}\right)\right) /(\hbar c) \\
& j_{M^{\prime \prime}}^{\prime \prime}=0,+1,-1,0 \text { for, respectively, } M^{\prime \prime}=0,1,2,3 \\
& d^{\prime \prime}=\left(2-\left(\log \left(m_{\mu} / m_{\epsilon}\right) / \log \left(\beta^{1 / 3}\right)\right)\right) \approx 3.840679 \times 10^{-2} \\
& 1-\delta\left(M^{\prime}, 3\right) \text { equals } 0 \text {, for } M^{\prime}=3 \text {, and equals } 1 \text {, otherwise } \\
& j_{M^{\prime}}^{\prime}=0,-1,0,+1 \text { for, respectively, } M^{\prime}=3,2,3 / 2,1 \\
& d^{\prime}(0) \sim 0.318 \\
& d^{\prime}(1) \sim-1.057 \\
& d^{\prime}(2) \sim-1.5091 \\
& m(1,3) \approx 8.59341 \mathrm{MeV} / c^{2}
\end{aligned}
$$

In equation (108), the factor $3 / 2$ correlates with the average of $M^{\prime}=2$ and $M^{\prime}=1$ and correlates with equation (107). (Note the appearance of $M^{\prime}=3 / 2$ in equation (113). The concepts of $M^{\prime}=3 / 2$ and $m\left(M^{\prime \prime}, 3 / 2\right)$ are useful mathematically, though not necessarily directly relevant to physics.) Regarding equations (114), (115), and (116), we choose values that fit data. (The relative signs of the three $d^{\prime}\left({ }_{-}\right)$ reflect the differences - regarding the positioning of quarks - between table 78 and table 79.) Regarding each charged lepton, our calculations fit data to more significant figures than the numbers in table 79 show. Regarding the tauon, our calculation correlates with a mass that may be more accurate, and more accurately specified, than the mass that references [13] and [14] show. (See equations (104) and (100).)

Table 80 shows ranges of $d^{\prime}\left(M^{\prime \prime}\right)$ that fit the data ranges that table 79 shows for quark masses. (See equations (114), (115), and (116).)

To the extent that people measure quark masses more accurately, people might find relationships between $d^{\prime}(0), d^{\prime}(1)$, and $d^{\prime}(2)$, and thereby reduce the number of parameters to less than seven. 
Table 81: Possible estimates for quark rest energies

\begin{tabular}{cccccc}
\hline$M^{\prime \prime}$ & Ratio & Value & $d^{\prime}\left(M^{\prime \prime}\right)$ & $m_{M^{\prime}=2} c^{2}(\mathrm{MeV})$ & $m_{M^{\prime}=1} c^{2}(\mathrm{MeV})$ \\
\hline 0 & $m_{\epsilon} m_{d} /\left(m_{u}\right)^{2}$ & $1 / 2$ & $\approx 0.31216454$ & $m_{u} c^{2} \approx 2.203 \times 10^{0}$ & $m_{d} c^{2} \approx 4.748 \times 10^{0}$ \\
1 & $m(1,3) m_{c} /\left(m_{s}\right)^{2}$ & 1 & -1 & $m_{c} c^{2} \approx 1.178 \times 10^{3}$ & $m_{s} c^{2} \approx 1.006 \times 10^{2}$ \\
2 & $m_{\mu} m_{t} /\left(m_{b}\right)^{2}$ & 1 & $-3 / 2$ & $m_{t} c^{2} \approx 1.695 \times 10^{5}$ & $m_{b} c^{2} \approx 4.322 \times 10^{3}$ \\
\hline
\end{tabular}

Table 81 speculates regarding possible rest energies for quarks. For each row, we assume the value that the third column shows for the ratio that the second column defines. The value implies the number that the column labeled $d^{\prime}\left(M^{\prime \prime}\right)$ shows. The six estimated quark rest energies might not be incompatible with experimental results that table 79 shows. To the extent that table 81 comports with nature, fitting the masses of six quarks and three charged leptons requires at most five parameters. The five parameters can be $m_{\epsilon}, m_{\mu}, \beta, \alpha$, and $d^{\prime}(0)$.

To the extent that table 81 comports with nature, equation 118 pertains.

$$
\left(m_{s}\right)^{2} m_{\mu}=m_{\epsilon} m_{\tau} m_{c}
$$

The charge $q_{\epsilon}$ correlates with $\beta$ via equation (102). The charge $q_{\epsilon}$ appears in $\alpha$, via equation (109). Based on equations (93) and (108) and based on modeling for the G-family, proposed modeling entangles concepts related to mass and concepts related to charge more deeply than does ongoing modeling.

Equations (119) and (120) explore the possibility for a relationship - perhaps similar to equation (102) - regarding the ratio $m_{\mu} / m_{\epsilon}$ or the ratio $m_{\tau} / m_{\mu}$. Equation (121) shows the result that we compute based on data from reference [13]. Equation (122) shows the result that we compute based on data from reference [14]. The main difference between the two sets of data lies in values of the gravitational constant, $G_{N}$. (The two references present the same value for the tauon mass. However, for each result, we use a tauon mass that is based on equation (102).) We do not explore possible significance for the notion that $1+x \approx 10 / 9$.

$$
\begin{gathered}
(1+x) \beta^{1 / 3}=m_{\tau} / m_{\mu} \approx m(1,3) / m_{\epsilon} \\
(1+x)^{-2} \beta^{1 / 3} \approx m_{\mu} / m(1,3) \\
x \approx 0.110033 \\
x \approx 0.110031
\end{gathered}
$$

\subsubsection{The relative strengths of electromagnetism and gravity}

We explore concepts that might correlate with the ongoing modeling notion that the strength of gravity is much less than the strength of electromagnetism.

We explore modeling for interactions that involve a charged simple fermion, such as an electron.

We assume that we can work within aspects of proposed modeling that de-emphasize translational motion and multicomponent objects. We assume that conservation of angular momentum pertains.

We correlate the symbol $1 \mathrm{~F}$ with that fermion. We explore interactions that model as if the number of incoming bosons equals the number of outgoing bosons. (Perhaps, compare with table 115. Note that, in table 115, a number $\mathrm{n}$ - such as in $\mathrm{nf}$ - denotes a number of particles and not a value of $2 S$. For example, $3 f$ denotes three elementary fermions.) Equation (123) shows an interaction in which the fermion absorbs a photon. The spin of the fermion flips. Angular momentum is conserved. Trying to replace, in equation (123), $2 \mathrm{G}$ with $4 \mathrm{G}$ does not work. The angular momentum associated with the fermion can change by no more than one unit. The interaction would not conserve angular momentum. Equation (124) can pertain. (Equation (124) does not portray an interaction - mediated by a $2 \mathrm{~J}$ boson - between two fermions. One can consider that the $2 \mathrm{~J}$ particle in equation (124) is a $2 \mathrm{~J}_{ \pm}$. See table 70 . One might want to consider the notion that equation (124) pertains regarding modeling and - in the current state of the universe does not necessarily pertain regarding easily directly observable physics.)

$$
\begin{aligned}
& 1 \mathrm{~F}+2 \mathrm{G} \rightarrow 1 \mathrm{~F}+0 \mathrm{I} \\
& 1 \mathrm{~F}+4 \mathrm{G} \rightarrow 1 \mathrm{~F}+2 \mathrm{~J}
\end{aligned}
$$


The notion that $1 \mathrm{~F}+4 \mathrm{G} \rightarrow 1 \mathrm{~F}+0 \mathrm{I}$ does not pertain might correlate with ongoing modeling notions that the strength of gravity is much less than the strength of electromagnetism.

We explore the strengths - for the monopole components of interactions between pairs of charged leptons - of electromagnetism and gravity. We use KMS Newtonian modeling.

For each of the three charged leptons, equation (125) characterizes the strength of the $2 \mathrm{G} 2$ component of electromagnetism. Here, $r$ denotes the distance between the two particles. Here, $F$ denotes the strength of the force. The equation correlates with a magnitude of the force. The interaction is repulsive. Equation (126) shows notation regarding the masses of charged leptons. (See discussion related to table 79.) Here, $\epsilon, \mu$, and $\tau$ denote respectively the electron, the muon, and the tauon. Here, the three in $m\left(M^{\prime \prime}, 3\right)$ correlates with charged leptons. (Compare with equation (108), which pertains to the masses of quarks and charged leptons.) Equation (127) repeats equation (101). Equation (128) shows results that reflect data. (We used data that reference [14] shows.) Equation (129) provides a $4 \mathrm{G} 4$ analog to the 2G2 equation (125). The symbol $G_{N}$ denotes the gravitational constant. The equation correlates with a magnitude of the force. Here, the interaction is attractive.

$$
\begin{gathered}
r^{2} F=\left(q_{\epsilon}\right)^{2} /\left(4 \pi \varepsilon_{0}\right) \\
m\left(M^{\prime \prime}, 3\right)=m_{x}, \text { for the pairs } M^{\prime \prime}=0, x=\epsilon ; M^{\prime \prime}=2, x=\mu ; \text { and } M^{\prime \prime}=3, x=\tau \\
\beta^{\prime}=m_{\tau} / m_{\epsilon} \\
m\left(M^{\prime \prime}, 3\right)=y_{M^{\prime \prime}}\left(\beta^{\prime}\right)^{M^{\prime \prime}} m_{\epsilon}, \text { with } y_{0}=y_{3}=1 \text { and } y_{2} \approx 0.9009 \\
r^{2} F=G_{N}\left(m\left(M^{\prime \prime}, 3\right)\right)^{2}
\end{gathered}
$$

We pursue the concept that a value of $M^{\prime \prime}$ can point to a relationship between the strength of electromagnetism and the strength of gravity. Based on the definitions just above, equation (130) pertains within experimental errors regarding relevant data. (Reference [13] provides the data.) Here, in essence, the equation $y_{18}=y_{0}=1$ pertains. Equation (130) echoes equation (102).

$$
\left(\left(q_{\epsilon}\right)^{2} /\left(4 \pi \varepsilon_{0}\right)\right) / 4=\left(G_{N}(m(18,3))^{2}\right) / 3, \text { with } m(18,3)=\left(\beta^{\prime}\right)^{6} m_{\epsilon}
$$

Proposed modeling interprets equation (130) as suggesting that the strength of $2 \mathrm{G} 2$ correlates with four so-called channels. (See discussion related to equation (134).) The interaction strength for each channel is $\left(\left(q_{\epsilon}\right)^{2} /\left(4 \pi \varepsilon_{0}\right)\right) / 4$. The strengths of the four channels combine by addition to yield the $2 \mathrm{G} 2$ strength $\left(q_{\epsilon}\right)^{2} /\left(4 \pi \varepsilon_{0}\right)$. Similarly, the expression $G_{N}\left(m\left(M^{\prime \prime}, 3\right)\right)^{2} / 3$ characterizes the strength per channel for $4 \mathrm{G} 4$. Here, the strengths of the three channels add to yield $G_{N}\left(m\left(M^{\prime \prime}, 3\right)\right)^{2}$.

The following notes pertain.

- Equation (130) links the ratio of two simple particle masses to a ratio of the strengths of two G-family force components.

- Equation (130) links the strength of $2 \mathrm{G} 2$ interactions to the strength of $4 \mathrm{G} 4$ interactions.

- Equation (131) correlates the fine-structure constant, $\alpha$, with a function of the tauon mass and the electron mass. (Regarding the fine-structure constant, see equation (109).)

$$
\alpha=\left(\left(q_{\epsilon}\right)^{2} /\left(4 \pi \varepsilon_{0} \hbar c\right)\right)=(4 / 3) \times\left(m_{\tau} / m_{\epsilon}\right)^{12} G_{N}\left(m_{\epsilon}\right)^{2} /(\hbar c)
$$

- Equation (132) characterizes a per channel ratio that pertains for interactions between two electrons.

$$
\left(\left(\left(q_{\epsilon}\right)^{2} /\left(4 \pi \varepsilon_{0}\right)\right) / 4\right) /\left(\left(G_{N}\left(m_{\epsilon}\right)^{2}\right) / 3\right) \approx 3.124 \times 10^{42}
$$

- Equation (133) recasts equation (102) to feature, in effect, the magnitudes of three interactions, with each one of the interactions involving two similar particles. (For example, $G_{N}\left(m_{\tau}\right)^{2}$ correlates with a gravitational interaction between two tauons.)

$$
(4 / 3)\left(\left(G_{N}\left(m_{\tau}\right)^{2}\right) /\left(G_{N}\left(m_{\epsilon}\right)^{2}\right)\right)^{6}=\left(\left(q_{\epsilon}\right)^{2} /\left(4 \pi \varepsilon_{0}\right)\right) /\left(G_{N}\left(m_{\epsilon}\right)^{2}\right)
$$


Table 82: Possibilities, based on concepts related to observations and proposed modeling pertaining to neutrinos

Two possibilities:

- If we assume that all three neutrinos are Dirac fermions, discussion related to equation (143) and table 84 points to the notion that the number of channels that pertains for each $8 \mathrm{G} 2468 \mathrm{x}$ equals the number of channels that pertains for each $4 \mathrm{G} 2468 \mathrm{x}$. (Here, $\mathrm{x}$ can be either one of a and b.) Equation (135) would pertain. The number of channels is one. The factor of three in equation (143) correlates with the number of Dirac neutrinos. (See discussion regarding table 84.)

- If we assume that one of the three neutrinos is a Dirac fermion and that the other two neutrinos are Majorana fermions, discussion related to equation (143) and table 84 points to the notion that the number of channels that pertains for each $8 \mathrm{G} 2468 \mathrm{x}$ equals one-third of the number of channels that pertains for each $4 \mathrm{G} 2468 \mathrm{x}$. Equation (134) would pertain. The factor of three in equation (143) correlates with a ratio of three $4 \mathrm{G}$ channels to one $8 \mathrm{G}$ channel.

\subsubsection{Channels and interactions that involve G-family bosons}

The notion of channels pertains to, at least, the relative strengths of the $2 \mathrm{G} 2$ component of $2 \mathrm{G}$ (or, electromagnetism) and the $4 \mathrm{G} 4$ component of $4 \mathrm{G}$ (or, gravity). (See discussion related to equation (130).) We extrapolate. For $6 \mathrm{G} 6$, the number of channels is two. For $8 \mathrm{G} 8$, the number of channels is one. For $\Sigma=10$ and $\Gamma=\llbracket 10 \rrbracket, \Sigma G \Gamma$ would correlate with zero channels and no interactions.

Each of equation (134) and equation (135) provides a candidate formula for the number of channels that pertain for a G-family solution $\Sigma G \Gamma$ that correlates with a G-family elementary particle. (This discussion does not necessarily pertain regarding the jay bosons and the $0 \mathrm{G} \emptyset$ solution. This discussion does not necessarily pertain regarding other - for example, the Higgs boson and the weak interaction bosons - elementary bosons that correlate with 0G $\mathrm{G}$ solutions.)

$$
\begin{gathered}
5-(\Sigma / 2) \\
5-\left(\lambda_{\max } / 2\right), \text { with } \lambda_{\max }=\max \{\lambda \mid \lambda \in \Gamma\}
\end{gathered}
$$

Equation (134) would correlate with excitations of the various $\Sigma$ G. (See, for example, table 48.) Possible G-family forces correlating with $\Sigma \geq 10$ would not be relevant to physics. (See table 23.) We would say that $\Sigma_{\max }=8$.

Equation (135) provides another possibility. Equation (135) correlates with notions of components $\Sigma$ GT. Equation (135) might correlate with TA-side symmetries. (See table 62.) Either one of $\Sigma_{\max }=8$ or $\Sigma_{\max }=20$ might pertain to nature.

Proposed modeling uses equation (135) to compute numbers of channels.

We elaborate regarding the selection of equation (135) to compute numbers of channels.

Regarding $2 \mathrm{G}$, some objects measure as having charge (which correlates with $2 \mathrm{G} 2$ ) and not having intrinsic magnetic fields (which would correlate with 2G24). Other objects measure as having intrinsic magnetic fields and not having net non-zero charge. We think that such notions point to the notion that channels correlate directly with $2 \mathrm{G} \Gamma$ and not directly with $2 \mathrm{G}$.

Table 82 summarizes a thought experiment. The notion that some neutrinos are Dirac fermions and some neutrinos are Majorana fermions seems less than fully appealing. (See, for example, table 29e,)

This essay uses equation (135) to compute numbers of channels.

\subsubsection{The relative strengths of 2G2, 2G24, W-boson, and other spin-one interactions}

We explore a possible relationship between the strength of electromagnetism correlating with G-family monopole interactions with charge and the strength of electromagnetism correlating with G-family dipole interactions with nominal magnetic dipole moment.

Equation (136) provides one definition of the fine-structure constant. (Compare with equation (109), which provides a more common definition.) In equation (136), $\left(q_{\epsilon}\right)^{2} /\left(4 \pi \varepsilon_{0} c\right)$ correlates with the strength of $2 \mathrm{G} 2$.

$$
\alpha=\left(\left(q_{\epsilon} / \hbar\right)^{2} /\left(4 \pi \varepsilon_{0} c\right)\right) \cdot \hbar
$$

Equation (136) provides a link between the strength of $2 \mathrm{G} 2$ and the strength of $2 \mathrm{G} 24$. The equation includes the term $\left(q_{\epsilon} / \hbar\right)^{2}$. The Josephson constant $K_{\mathrm{J}}$ equals $2 q_{\epsilon} / h$ (or, $q_{\epsilon} /(2 \pi \hbar)$ ). Ongoing modeling considers that magnetic flux is always an integer multiple of $h /\left(2 q_{\epsilon}\right)$. (We note the existence of an analog 
- to equation (136) - for which $\alpha=(\cdots) \cdot K_{\mathrm{J}}$. Elsewhere, this essay links spin to aspects pertaining to the squares of masses of elementary bosons. See, for example, discussion related to equation (93) and discussion related to equation (95). Elsewhere, this essay mentions the notion that aspects pertaining to squares of masses of elementary bosons might link with nominal magnetic dipole moment. See, discussion related to equation (93). Possibly, the $\alpha=(\cdots) \cdot K_{\mathrm{J}}$ analog to equation (136) has relevance to aspects pertaining to squares of masses of elementary bosons. This essay does not further discuss possible relevance of the $\alpha=(\cdots) \cdot K_{\mathrm{J}}$ analog to equation (136).)

We explore a concept regarding ongoing modeling notions that correlate with relationships between the strengths of the electromagnetic, weak, and strong interactions.

We use the symbol $\Sigma \mathrm{B}$ to denote an elementary boson having a spin of $\Sigma / 2$. The expression $1 \mathrm{~F}+2 \mathrm{~B} \rightarrow 1 \mathrm{~F}+0 \mathrm{~B}$ can pertain for each of the following cases $-2 \mathrm{~B}$ correlates with $2 \mathrm{G}, 2 \mathrm{~B}$ correlates with $2 \mathrm{~W}$, and $2 \mathrm{~B}$ correlates with $2 \mathrm{U}$. This notion might correlate with ongoing modeling notions that correlate with relationships between the strengths of the electromagnetic, weak, and strong interactions.

\subsubsection{Relative strengths of interactions re G-family bosons with spins of at least two}

Equations (137) and (138) parallel equation (124). Compared to equation (124), equation (137) requires dissipation - from the incoming G-family boson - of one more unit - of magnitude $\hbar$ - of spin. Compared to equation (137), equation (138) requires dissipation - from the incoming G-family boson of one more unit - of magnitude $\hbar$ - of spin.

$$
\begin{gathered}
1 \mathrm{~F}+6 \mathrm{G}+0 \mathrm{I} \rightarrow 1 \mathrm{~F}+2 \mathrm{~J}+2 \mathrm{~J} \\
1 \mathrm{~F}+8 \mathrm{G}+0 \mathrm{I}+0 \mathrm{I} \rightarrow 1 \mathrm{~F}+2 \mathrm{~J}+2 \mathrm{~J}+2 \mathrm{~J}
\end{gathered}
$$

Each of 4G4, 6G6, and $8 \mathrm{G} 8$ interacts with a different property of objects. In effect, $4 \mathrm{G} 4$ interacts with (at least some) elementary fermions, while neither of 6G6 and 8G8 interacts with elementary fermions. (See table 93.)

We explore the notion that a strength scaling relationship might pertain regarding G-family components $\Sigma G \Gamma$ that share a value of $\Gamma$. For two such $\Sigma G \Gamma, \Sigma_{1} G \Gamma$ and $\Sigma_{2} G \Gamma$, equation $(139$ pertains.

$$
\left|\Sigma_{2}-\Sigma_{1}\right| / 4 \text { is an integer }
$$

We interpret equation (136) as suggesting that a factor of $\alpha$ might pertain regarding modeling the absorbing of a unit of spin. For a step from equation (124) to equation (138), two factors of $\alpha$ would pertain. The factor of $\alpha^{2}$ correlates with the notion that some proposed modeling suggests $\alpha^{2} m_{\epsilon}$ as equaling or being somewhat less than the ongoing modeling average neutrino mass. (See equation (143).)

We explore aspects that would be relevant for the case of $\Sigma_{\max }=20$ but not for the case of $\Sigma_{\max }=8$.

Each one of $10 \mathrm{G}, 12 \mathrm{G}, \ldots$, and $20 \mathrm{G}$ would interact with anomalous properties and not with nominal properties. Examples of ongoing modeling nominal properties include charge (which correlates with a proposed modeling component, $2 \mathrm{G} 2$, of $2 \mathrm{G}$ ), nominal magnetic dipole moment (which correlates with a proposed modeling component, $2 \mathrm{G} 24$, of $2 \mathrm{G}$ ), and rest mass (which correlates with a proposed modeling component, $4 \mathrm{G} 4$, of $4 \mathrm{G}$ ). An example of an ongoing modeling anomalous property is anomalous magnetic dipole moment. Detecting effects of $\Sigma \mathrm{G}$ for which $\Sigma \geq 10$ might be difficult. (See discussion related to equation (140).)

For each G-family physics-relevant $\Sigma$ G $\Gamma$ solution for which $\Sigma \geq 10$, there is a G-family physics-relevant $\Sigma^{\prime} \mathrm{G} \Gamma$ solution for which $\Sigma^{\prime}$ is less than $\Sigma$. (Compare table 92c with the combination of table 92a and table 92b.) Equation (140) pertains. Also, each G-family physics-relevant $\Sigma$ G $\Gamma$ solution for which $\Sigma \geq 10$ correlates with one of the words dipole, quadrupole, or octupole.

$$
\Sigma-\Sigma^{\prime} \geq 4
$$

Equation (141) follows from equation (109).

$$
\alpha^{2}<5.33 \times 10^{-5}
$$

Effects correlating with each G-family physics-relevant $\Sigma G \Gamma$ solution for which $\Sigma \geq 10$ might be difficult to observe. For each one of those solutions, the word monopole does not pertain and there is a relevant G-family $\Sigma^{\prime} \mathrm{G} \Gamma$ solution (for which the same word out of the list dipole, quadrupole, and octupole pertains) that contributes an effect that is at least a factor of $10^{4}$ larger than effects of the $\Sigma G \Gamma$ solution. 
Table 83: Possible relevance - regarding some interaction strengths - of the fine-structure constant (with the symbol $\mathrm{O}$ denoting the two-word term ongoing modeling; with the symbol $\mathrm{P}$ denoting the two-word term proposed modeling; with KMS denoting KMS modeling; with PFS denoting PFS modeling; with QED denoting the two-word term quantum electrodynamics; and with the symbol * denoting the expression $\left.\left(1-\delta\left(M^{\prime}, 3\right)\right) \cdot\left((3 / 2) \cdot\left(1+M^{\prime \prime}\right)+\left(j_{M^{\prime}}^{\prime}\right) d^{\prime}\left(M^{\prime \prime}\right)\right)\right)$

\begin{tabular}{|c|c|c|c|}
\hline $\mathrm{P} / \mathrm{O}$ & Context & Aspect & Factor \\
\hline $\mathrm{O}-\mathrm{KMS}$ & $\begin{array}{l}\text { QED calculations of anomalous } \\
\text { magnetic dipole moments }\end{array}$ & $\begin{array}{l}\text { Terms involving } j \text { virtual photons (See } \\
\text { discussion related to equation (193).) }\end{array}$ & $\alpha^{j}$ \\
\hline P - PFS & $\begin{array}{l}\text { Relationship between the masses } \\
\text { of quarks and the masses of } \\
\text { charged leptons }\end{array}$ & (See equation (108).) & $\left(\alpha^{-1 / 4}\right)^{*}$ \\
\hline P - PFS & $\begin{array}{l}\text { Possible relationship between } \\
\text { the strength of } 2 \mathrm{G} 2 \text { and the } \\
\text { strength of } 2 \mathrm{G} 24\end{array}$ & $\begin{array}{l}\text { (See discussion related to equation } \\
(136) .)\end{array}$ & $\alpha$ \\
\hline P - PFS & $\begin{array}{l}\text { Lepton number and (ongoing } \\
\text { modeling) perceived masses for } \\
\text { neutrinos }\end{array}$ & $\begin{array}{l}\text { Ratio of strengths: } 4 \mathrm{G} 2468 \mathrm{x} \text { to } 8 \mathrm{G} 2468 \mathrm{x} \text {, } \\
\text { with } \mathrm{x} \text { equal to a or b (See discussion } \\
\text { related to equation (142). See table } 84 \text {. } \\
\text { See discussion related to equation (138).) }\end{array}$ & 1 to $\alpha^{2}$ \\
\hline P - PFS & $\begin{array}{l}\text { Possible generalization for } \\
\Sigma \geq 4,2 \in \Gamma \text {, and } j>0\end{array}$ & $\begin{array}{l}\text { Ratio of strengths: } \Sigma \mathrm{G} \Gamma \text { to }(\Sigma+2 j) \mathrm{G} \Gamma \\
\text { (See discussion related to equation } \\
\text { (138).) }\end{array}$ & 1 to $\alpha^{j}$ \\
\hline P - PFS & $\begin{array}{l}\text { Possible (speculative) aspects } \\
\text { regarding the mass of the } \mathrm{W} \\
\text { boson. }\end{array}$ & $\begin{array}{l}\text { (See discussion related to table } 76 \text { and } \\
\text { discussion related to equation (136).) }\end{array}$ & $?$ \\
\hline
\end{tabular}

\subsubsection{Roles of the fine-structure constant}

Table 83 notes some possibilities for relevance of the fine-structure constant regarding ratios of strengths of interactions.

\subsubsection{Numbers of fermion generations}

Unverified ongoing modeling includes notions of a fourth generation of neutrino. People use the two-word term sterile neutrino. We know of no data that supports the existence of a fourth neutrino.

Table 58 points to proposed modeling aspects that correlate with exactly three generations for elementary fermions. For modeling for elementary fermions, the $S U(2)$ symmetry that correlates with the SA5-and-SA6 oscillator pair correlates with three generators. For example, regarding table 45 , the three (SA-side) generators correlate with three fermion generations. The matching TA5-and-TA6 $S U(2)$ symmetry correlates with rebuttable somewhat conservation of fermion generation.

\subsubsection{Ongoing modeling estimates for the sum of neutrino masses}

Equation (142) provides ongoing modeling limits for the sum, across three generations, of neutrino masses. (See reference [13]. Reference [15] provides the lowest of the upper limits that reference [13] lists.) The integer $j$ correlates with generation. Equation (142) comes from interpretations of astrophysics data.

$$
0.06 \mathrm{eV} / c^{2} \lesssim \sum_{j=1}^{3} m_{j} \lesssim 0.12 \mathrm{eV} / c^{2}
$$

Independent of results of observations and of assumptions about modeling, equation (143) pertains.

$$
3 \alpha^{2} m_{\epsilon} \approx 0.0816 \mathrm{eV} / c^{2}
$$

We anticipate exploring notions that the following sentences state. The number $3 \alpha^{2} m_{\epsilon}$ might predict an upper bound for the lower limit of the range that people derive from the types of observations that underlie equation (142). The number $3 \alpha^{2} m_{\epsilon}$ might predict a lower bound for the upper limit of the range that people derive from the types of observations that underlie equation (142). The factor of three might correlate with the range $1 \leq j \leq 3$ in equation (142). 
Table 84: Interpretations regarding some aspects of G-family solutions

\begin{tabular}{cl}
\hline Aspect & Interpretation \\
\hline $8 \mathrm{G}$ & Interacts with lepton number minus baryon number \\
8G2468a and 8G2468b & Interact with individual neutrinos \\
8G2468a and 8G2468b & Catalyze neutrino oscillations \\
8G2468a and 8G2468b & $\begin{array}{l}\text { Catalyze effects that people interpret as implying (via ongoing } \\
\text { modeling) that at least one generation of neutrino has non-zero } \\
\text { mass }\end{array}$ \\
\hline
\end{tabular}

Table 85: Aspects relevant to modeling regarding magnitudes of neutrino oscillations

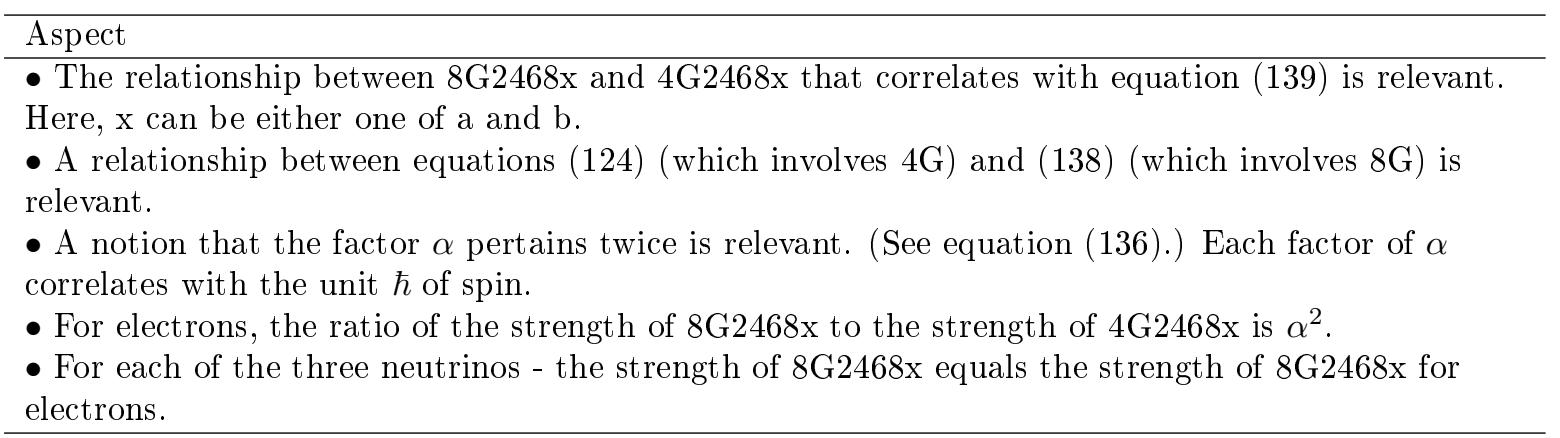

\subsubsection{Models that might estimate an ongoing modeling sum of neutrino masses}

We explore possibilities for developing models that would estimate a non-zero ongoing modeling sum of neutrino masses.

One possibility has bases in the notion that one can extrapolate, based on equation (108), to results that pertain to neutrino masses. We do not find a seemingly useful method. We de-emphasize this possibility.

One possibility assumes the ongoing modeling notion that neutrino oscillations correlate with interactions that we correlate with the $4 \mathrm{G}$ subfamily. Table $48 \mathrm{a}$ correlates $4 \mathrm{G}$ with rebuttable somewhat conservation of fermion generation. Neutrino oscillations might correlate with virtual interactions with $4 \mathrm{G}$ or with multiple close-by interactions with $4 \mathrm{G}$. (People might, therefore, correlate neutrino oscillations with notions of CP violation. See discussion related to table 42.) However, such interactions might not account for observed magnitudes of neutrino oscillations. (Also, ongoing modeling seems not to propose a correlation between CP violation and neutrino oscillations.) We de-emphasize the possibility that quantum interaction with $4 \mathrm{G}$ accounts for most of the effects that people correlate with neutrino oscillations.

One possibility has bases in the notion that modeling regarding neutrino oscillations might feature notions of indices of refraction. (See discussion related to equation (78).) Some refraction might correlate with ongoing modeling notions of classical physics interactions with gravity. As far as we know, people have yet to detect (gravitational lensing of neutrinos or) gravitational refraction of neutrinos. (See discussion related to table 86.) Proposed modeling suggests that interactions mediated by $8 \mathrm{G}$ bosons play significant roles regarding refraction of neutrinos.

Matter charged leptons, including the electron, and matter neutrino simple particles correlate with the same $3 \mathrm{LB}$ number $-\iota_{3 L B}=3$. We assume that, here, neutrinos model as being Dirac fermions and not as being Majorana fermions. (For Majorana fermions, $\iota_{3 L B}$ would be zero. Perhaps, see table 29e.)

Table 84 points to notions that might underlie modeling regarding neutrino oscillations.

Table 85 lists aspects that we posit to have relevance. Here, we posit that - regarding the strength of 8 G2468x interactions with neutrinos - the mass of the muon and the mass of the tauon are not relevant. (Perhaps, the notion of rest energy minus freeable energy pertains. Perhaps, that notion correlates with the notion that, for $\Sigma G 2468 x$, each of $4 \in \Gamma$ and $6 \in \Gamma$ pertains.)

We discuss possible implications regarding ongoing modeling.

Ongoing modeling astrophysics modeling does not include modeling that proposed modeling correlates with $6 \mathrm{G}$ and $8 \mathrm{G}$. We posit one or two conceptual mapping steps. First, in the context of proposed modeling, modeling for $8 \mathrm{G}$ octupole components of force maps to modeling for octupole components of $4 \mathrm{G}$ forces. Perhaps that step suffices. In this context, ongoing modeling paralleling aspects of proposed modeling $4 \mathrm{G} 2468 \mathrm{a}$ and $4 \mathrm{G} 2468 \mathrm{~b}$ interprets $8 \mathrm{G}$ effects on neutrinos as correlating with non-zero neutrino 
Table 86: Aspects that might correlate with the extent to which neutrinos have non-zero masses

Aspect

- Limits regarding neutrino masses, as inferred from astrophysics data.

- The existence of neutrino oscillations.

- Neutrino speeds.

- Effects of neutrino lensing (which would be based on gravity).

- Other.

mass. The following (or, second) step pertains to the extent that relevant ongoing modeling does not correlate adequately well with proposed modeling non-monopole components of $4 \mathrm{G}$. In the context of proposed modeling, modeling for $4 \mathrm{G}$ octupole components of force maps to modeling involving $4 \mathrm{G} 4$. In this context, ongoing modeling based on only proposed modeling $4 \mathrm{G} 4$ would interpret $8 \mathrm{G}$ effects on neutrinos as correlating with non-zero neutrino mass.

We perform a check regarding the reasonableness of proposed modeling regarding interactions that couple to lepton number. (Here, KMS modeling pertains.)

We consider our interpretation of aspects of ongoing modeling. We consider gravitational interactions between two electrons. Equation (144) describes results based just on the component that correlates with proposed modeling 4G4 effects. Equation (145) assumes that $\epsilon^{\prime}$ correlates with one standard deviation regarding the mass of an electron. (Reference [13] provides the data that we use for these calculations.) The lepton number for an electron equals the lepton number for a matter neutrino. Equation (146) correlates with results based just on the component that correlates with proposed modeling $8 \mathrm{G}$ effects. (One exponent of two correlates with the exponent of two pertaining, in essence, to equation (143). One exponent of two correlates with the notion that the interaction involves two simple fermions.) The result that equation (146) shows is less than the result that equation (145) shows. In this context of ongoing modeling, the proposed modeling interaction, between two electrons, based on lepton number is not incompatible with measurements of electron masses.

$$
\begin{gathered}
G_{N}\left(m_{\epsilon}\left(1+\epsilon^{\prime}\right)\right)^{2} / r^{2} \approx G_{N}\left(m_{\epsilon}\right)^{2}\left(1+2 \epsilon^{\prime}\right) / r^{2} \\
\left|\epsilon^{\prime}\right| \approx 1.2 \times 10^{-8} \\
\left(\alpha^{2}\right)^{2} \approx 2.8 \times 10^{-9}
\end{gathered}
$$

Proposed modeling suggests that, for KMS Newtonian modeling, the strength of interactions with lepton number scales as $r^{-5}$. The strength of interactions with charge scales as $r^{-2}$. People might want to estimate a minimum energy for which the interaction between two charged leptons exhibits measurable effects of $8 \mathrm{G}$ octupole components.

We summarize proposed modeling suggestions about ongoing modeling statements about the sum of neutrino masses.

The following statements pertain. The dominant contribution to the relevant astrophysics data correlates with neutrino refraction based on interactions mediated by the $8 \mathrm{G} 2468 \mathrm{a}$ and $8 \mathrm{G} 2468 \mathrm{~b}$ components of $8 \mathrm{G}$. Contributions correlating with trajectory bending via classical physics refractive interactions with gravity might pertain. Contributions correlating with CP-violating interactions with gravity might pertain. Contributions correlating with non-zero neutrino masses might pertain. Each one of the three neutrinos might have zero mass.

\subsubsection{Neutrino masses}

Discussion related to table 84 suggests that proposed modeling can be compatible with modeling that is compatible with either one of the following two statements. All neutrinos have zero mass. Some neutrinos have non-zero mass.

We explore the notion that all neutrinos have zero mass, even though people interpret data as suggesting that at least one flavor of neutrino correlates with non-zero mass.

Table 86 lists aspects that might correlate with the extent to which neutrinos have non-zero masses.

We discuss inferences from astrophysics data.

Discussion related to table 84 and to equation (143) suggests modeling that would be compatible with data and with elementary particle Standard Model ongoing modeling aspects that suggest that all neutrinos have zero rest masses.

We discuss aspects related to neutrino oscillations. 
Ongoing modeling hypothesizes that gravity catalyzes neutrino oscillations. This hypothesis might correlate with a process of elimination. Ongoing modeling suggests that each known simple particle does not catalyze neutrino oscillations. Ongoing modeling suggests that photons do not catalyze neutrino oscillations. Ongoing modeling suggests that the strong interaction does not catalyze neutrino oscillations. The only ongoing modeling catalyst for neutrino oscillations might be gravity.

Proposed modeling suggests that $4 \mathrm{G}$ correlates with rebuttable somewhat conservation of fermion generation. Proposed modeling suggests that interactions mediated by $4 \mathrm{G}$ bosons might be insufficient to catalyze known amounts of neutrino oscillations. Proposed modeling suggests that $8 \mathrm{G}$ bosons catalyze observed neutrino oscillations. (See discussion related to table 84.)

We know of no data about neutrino speeds that would settle the question as to the extent to which neutrinos have non-zero masses.

As far as we know, observations of impacts of possible neutrino lensing have yet to produce relevant results.

As far as we know, other possibly relevant experiments and observations do not provide additional insight about the extent to which neutrinos have non-zero masses. (See, for example, references [16] and [17.)

Proposed modeling suggests that each neutrino might correlate with zero rest mass.

\subsubsection{A possible lack of electric dipole moments for elementary particles}

Table 62 points to no G-family solutions that would correlate with a non-zero electric dipole moment for a point-like elementary particle. The lack of such G-family solutions might correlate with nature not including elementary particles that have non-zero electric dipole moments.

\subsubsection{A possible lack of neutrino asymmetry}

Reference [18] suggests that people might be on the verge of finding an asymmetry, which would correlate with CP violation, between matter neutrinos and antimatter neutrinos. The article suggests that ongoing modeling interpretation of data seems to point toward such an asymmetry and that it might be reasonable to anticipate that, with more data, people will conclude that the asymmetry exists.

Proposed modeling offers an alternative explanation for such data.

People produce the relevant neutrinos 295 kilometers from where the measurements take place. Between production and detection, the neutrinos pass through earth. Along the path, if one just considers protons in atomic nuclei and electrons in materials, $\iota_{3 L B}$ is essentially zero. If one considers also the neutrons in atomic nuclei, $\iota_{3 L B}$ is negative. Core proposed modeling suggests that, via ongoing modeling virtual interactions, relevant neutrinos interact via $8 \mathrm{G}$ interactions with an $\iota_{3 L B}$ that is negative essentially everywhere along the relevant path. (Some aspects of the virtual interactions might correlate with $8 \mathrm{G} 2468 \mathrm{a}$ and $8 \mathrm{G} 2468 \mathrm{~b}$. To the extent that $8 \mathrm{G} 8$ pertains, the $8 \mathrm{G} 8$ component of the virtual interactions might have a magnitude that correlates with $\alpha^{2}$ times the strength of interactions between electrons and gravity.)

This explanation suggests that the would-be asymmetry might correlate with the material through which the neutrinos pass. This explanation suggests that the would-be asymmetry would not necessarily correlate with a $\mathrm{CP}$ violation asymmetry pertaining to neutrinos themselves.

\section{Results: astrophysics and cosmology}

This unit describes dark matter particles. This unit predicts and explains data about dark matter, galaxy formation, other aspects of astrophysics, and the cosmos.

\subsection{Summary: a table of predictions and explanations re astrophysics and cosmology}

We discuss aspects of nature - correlating with the terms dark matter, dark energy, astrophysics, and cosmology - for which proposed modeling might provide, relative to ongoing modeling, new details or better-defined explanations.

Table 87 lists some topics for which proposed modeling seems to provide insight that augments insight that ongoing modeling suggests.

We discuss immediately below some, but not all, of the items that table 87 lists.

Ongoing modeling explores various hypotheses regarding the fundamental components of dark matter. Proposed modeling suggests specific components for dark matter. Proposed modeling uses its description of dark matter fundamental components to explain data that ongoing modeling seems not to explain. 
Table 87: Aspects of nature - that ongoing modeling discusses or suggests - for which proposed modeling seems to provide insight that augments insight that ongoing modeling suggests

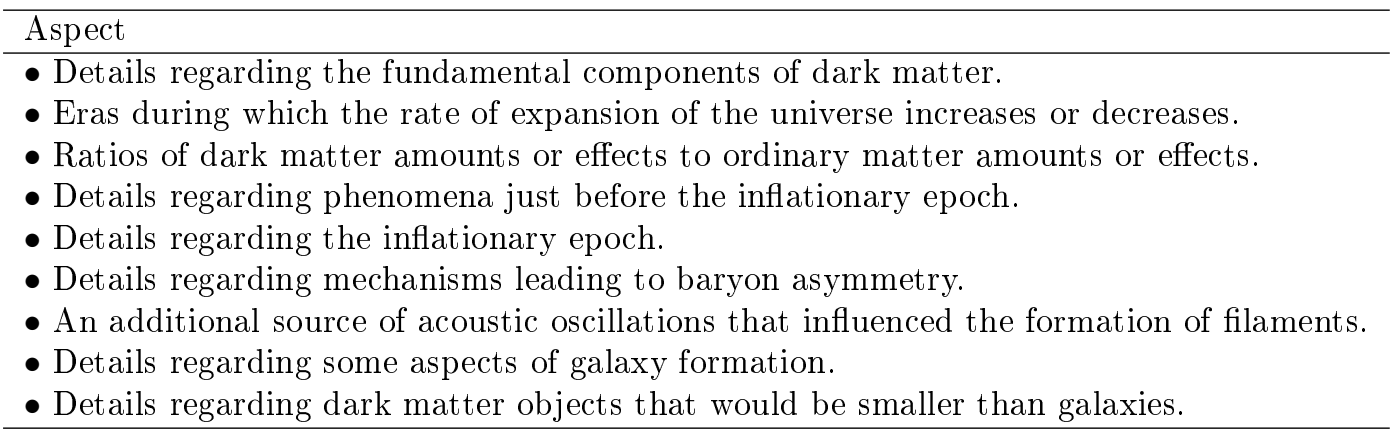

Ongoing modeling suggests notions regarding three known eras in the rate of expansion of the universe. One era features an accelerating (or, increasing) rate and correlates with the so-called inflationary epoch. A later multi-billion-year era features a decelerating (or, decreasing but still positive) rate. The current multi-billion-year era features an accelerating rate. Proposed modeling suggests an explanation that has bases in components of $4 \mathrm{G}$ forces. The explanation does not necessarily depend on ongoing modeling notions of dark energy negative pressure or on ongoing modeling models that have bases in general relativity. The proposed modeling explanation might be generally compatible with ongoing modeling models. The proposed modeling explanation points to some subtleties that ongoing modeling might miss.

Ongoing modeling seems not to explain patterns regarding ratios of dark matter to ordinary matter. Observations point to ratios of five-plus to one regarding densities of the universe and regarding amounts in galaxy clusters. Observations regarding ratios of amounts in early galaxies seem to cluster around zero-plus to one and four to one. Observations regarding ratios of amounts in later galaxies seem to cluster around zero-plus to one, four to one, and one to zero-plus. Observations regarding depletion, via hyperfine interactions with hydrogen atoms, of cosmic microwave background radiation (or, CMB) may point to a ratio of one to one. Proposed modeling suggests explanations for each of these ratios. (See discussion regarding table 95 and see, for example, table 106.) The explanations have bases in proposed modeling specifications for dark matter and in effects correlating with PFS modeling, with components of $4 \mathrm{G}$ forces, and with components of $2 \mathrm{G}$ forces. (See, for example, discussion related to tables 92, 93, 94 and 95.)

Ongoing modeling suggests that the early universe includes an inflationary epoch. Ongoing modeling proposes a role, during that epoch, for a so-called inflaton particle. Proposed modeling suggests that jay (or, 2J) bosons played key roles just before the inflationary epoch. One such role correlates with producing aye (or, 0I) bosons. Proposed modeling suggests that the aye simple particle correlates with the notion of an inflaton. Proposed modeling suggests that octupole components of $4 \mathrm{G}$ forces provided for rapid expansion.

Ongoing modeling suggests that the achievement of baryon asymmetry occurred after the formation of the universe. Ongoing modeling proposes mechanisms that might have catalyzed baryon asymmetry. Ongoing modeling does not necessarily point to the tweak simple bosons that proposed modeling suggests exist. Proposed modeling suggests that tweak bosons might have catalyzed the achievement of baryon asymmetry.

Ongoing modeling provides hypotheses regarding possibilities for substantial objects that might be significantly smaller than galaxies and contain mostly dark matter. Proposed modeling suggests some specifics regarding some objects that would be significantly smaller than galaxies and would contain mostly dark matter.

\subsection{Modeling pertaining to astrophysics and cosmology}

We discuss concepts and methods that lead to proposed modeling results regarding astrophysics and cosmology.

\subsubsection{Modeling that describes dark matter particles}

We discuss one type of dark matter.

We introduce the symbols that equations (147) and (148) show. The symbol 1Q $\otimes 2 \mathrm{U}$ denotes a particle that includes just quarks and gluons. The word hadron pertains for the particle. The one-element term hadron-like pertains for the particle. Examples of such particles include protons, neutrons, and pions. The 
symbol $1 \mathrm{R} \otimes 2 \mathrm{U}$ denotes a particle that includes just arcs and gluons. The one-element term hadron-like pertains for the particle. The particle does not include quarks.

$1 \mathrm{Q} \otimes 2 \mathrm{U}$

$1 \mathrm{R} \otimes 2 \mathrm{U}$

A $1 \mathrm{R} \otimes 2 \mathrm{U}$ hadron-like particle contains no charged simple particles. The $1 \mathrm{R} \otimes 2 \mathrm{U}$ hadron-like particles do not interact with $2 \gamma$. The $1 \mathrm{R} \otimes 2 \mathrm{U}$ hadron-like particles measure as being dark matter.

We correlate work above with the two-element term PR1ISP modeling.

The existence of $1 \mathrm{R} \otimes 2 \mathrm{U}$ hadron-like particles seems insufficient to explain ratios of dark matter effects to ordinary matter effects of (for example) five-plus to one, four to one, and maybe one to one.

We explore the notion that some five-plus to one ratios reflect something fundamental in nature. We correlate some results from this exploration with PR6ISP modeling. (See table 21b.)

The symbol element PR denotes the one-element term physics-relevant. The symbol element ISP correlates with the four-word term isomers of simple particles. The notion of isomer correlates with one so-called SM6_symmetry. (See table 21b.) Discussion related to table 98 and table 99 posits aspects that might correlate with differences between relevant isomers. (This proposed modeling notion of isomers does not necessarily correlate with ongoing modeling notions of isomers. This proposed modeling notion of isomers does not necessarily parallel the nuclear physics notion - same numbers of protons and neutrons, but different energy states - of isomer. This proposed modeling notion of isomers does not necessarily correlate with the chemistry notion - same numbers of various atoms, but different spatial arrangements - of molecular isomers.)

PFS modeling correlates interactions with charge with the $2 \mathrm{G} 2$ component of the $2 \mathrm{G}$ force. We posit that nature includes six isomers of charge. PFS modeling correlates interactions with nominal magnetic dipole moment with the $2 \mathrm{G} 24$ component of the $2 \mathrm{G}$ force. We posit that each isomer of charge correlates with one isomer of nominal magnetic dipole moment. We posit that each of the six pairings of one isomer of charge and one isomer of nominal magnetic moment correlates with its own isomer of all simple particles. One isomer of charge, nominal magnetic dipole moment, and related simple particles measures as ordinary matter. (The previous sentence also pertains regarding PR1ISP modeling.) We label that isomer as isomer zero. We posit that each of the other five isomers of charge, nominal magnetic dipole moment, and related simple particles measures as dark matter. (PR1ISP modeling does not include these five isomers.) We label those isomers as isomer one, isomer two, ..., and isomer five. We posit that each of the six isomers correlates with its own $2 \mathrm{U}$ particles (or, gluons). We posit that one isomer of $4 \mathrm{G} 4$ interacts with each one of the one ordinary matter isomer and five dark matter isomers.

We posit that the next two sentences pertain. The six-isomer notion explains the five that pertains regarding five-plus to one ratios of amounts of dark matter to ordinary matter. The existence of isomerzero $1 \mathrm{R} \otimes 2 \mathrm{U}$ hadron-like particles explains the plus that pertains regarding five-plus to one ratios of amounts of dark matter to ordinary matter. Such five-plus to one ratios pertain regarding densities of the universe and regarding the compositions of (perhaps most) galaxy clusters.

Table 88 provides perspective regarding PR6ISP modeling. The following sentences illustrate the last item in the table. People suggest that dark matter could have characteristics similar to ordinary matter. (See, for example, reference [19.) People suggest that dark matter might include components that include quarks or that might experience Yukawa-like potentials. (See, for example, references 20] and [21.) People suggest that nature might include dark matter photons. (See, for example, reference [22].)

Regarding each one of the six PR6ISP isomers, we suggest that each combination - that table 79 shows - of magnitude of charge and magnitude of mass pertains to a simple fermion that correlates with the isomer. For example, each isomer includes a charged lepton for which the magnitude of charge equals the magnitude of the charge of the ordinary matter electron and for which the rest energy equals the rest energy of the electron. However, regarding charged leptons, the combination of mass and generation number does not necessarily match across isomers. (See table 98.) For example, for so-called isomer one, the generation three charged lepton may have the same mass as the ordinary matter electron. (See table 79.) The ordinary matter electron has a generation number of one.

Tables $21 \mathrm{~b}$ and $27 \mathrm{~d}$ discuss the symbol $\iota_{I}$. Discussion just above pertains regarding $\operatorname{PR} \iota_{I} \mathrm{ISP}$, with $\iota_{I}$ being one or six. Within any one $\mathrm{PR} \iota_{I} \mathrm{ISP}$, equation (149) pertains for each simple particle, for each component of G-family force, for each U-family particle, and for each hadron-like particle. For example, for PR6ISP modeling, for the electron, the number of isomers is six and the span of each isomer is one. 
Table 88: Perspective regarding PR6ISP modeling

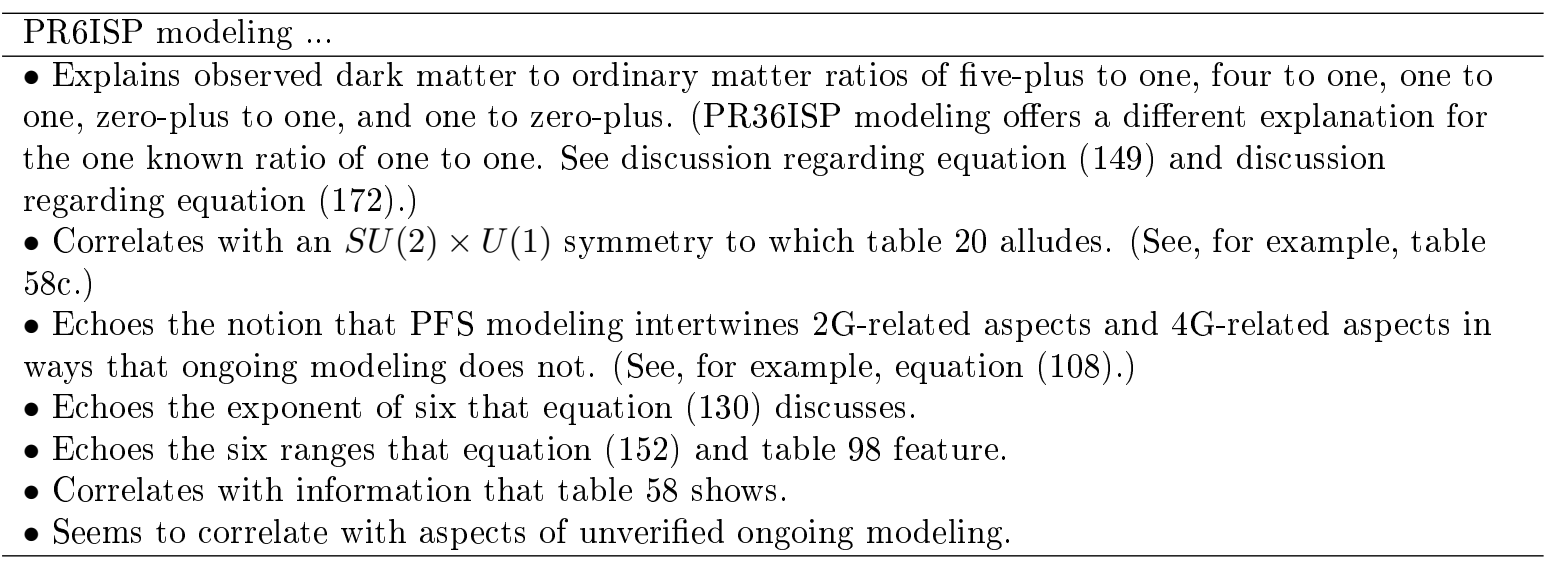

For PR6ISP modeling, for the 4G4 component of 4G, the number of isomers is one and the span of each isomer is six.

$$
\text { (number of isomers }) \times(\text { span of one isomer })=\iota_{I}
$$

PR6ISP modeling suggests that 2G248 has a span of six isomers of simple particles.

PR6ISP modeling assumes that the span of six for $2 \mathrm{G} 248$ embraces the same six isomers as does the span of six for $4 \mathrm{G} 4$.

We explore the notion that nature might include 36 isomers. (See table 21b.)

PR36ISP modeling embraces the possibility that the span of six for $2 \mathrm{G} 248$ is, in effect, orthogonal (or, perpendicular) to the span of six for $4 \mathrm{G} 4$. Here, six isomers of $4 \mathrm{G} 4$ pertain. Each of those six isomers of $4 \mathrm{G} 4$ spans a different (from the other five isomers of $4 \mathrm{G} 4$ ) six isomers of simple particles. We use the two-element term doubly-dark matter to correlate with the 30 isomers of simple particles that do not interact with the ordinary matter isomer via $4 \mathrm{G} 4$. Doubly dark matter does not interact with ordinary matter via $2 \mathrm{G} 2,2 \mathrm{G} 24,4 \mathrm{G} 4$, or other components of $4 \mathrm{G}$. Five doubly dark matter isomers of simple particles interact with the ordinary matter isomer via $2 \mathrm{G} 248$.

From the perspective of each one of the 36 isomers of simple particles, the following statements pertain. The isomer correlates with its own isomers of 2G2, 2G24, 4G246, 4G2468a, and 4G2468b. The isomer interacts via $2 \mathrm{G} 248$ with five other isomers. The isomer interacts via $4 \mathrm{G} 4$ with five other isomers. None of the first five other isomers is one of the second five other isomers. The first five other isomers are from the perspective of the one isomer - doubly dark matter isomers. The second five other isomers are - from the perspective of the one isomer - dark matter isomers.

Compared to PR6ISP modeling, PR36ISP modeling correlates with an additional six-generator symmetry. (See table 21b.)

We preview features of each of PR1ISP, PR6ISP, and PR36ISP modeling.

Table 89 discusses cumulative features of various types of modeling. Generally, each row augments the rows above that row. Regarding ongoing modeling, the symbol NR denotes the concept that the notion of isomers is not relevant. We think that PR6ISP provides useful insight about nature. Regarding ratios of dark energy density of the universe to density of the universe of ordinary matter plus dark matter, PR36ISP offers an alternative (to PR6ISP) explanation of dark energy density. (See discussion related to equation (172).) Otherwise, regarding bases for aspects that table 89 lists, PR36ISP is similar to PR6ISP. Discussion related to equation (172) suggests that PR6ISP modeling might suffice to explain known phenomena and that it might not be necessary to consider PR36ISP modeling. From a standpoint of observations, distinguishing between the case of PR6ISP and the case of PR36ISP might prove difficult.

Table 90 shows relationships regarding PR1ISP, PR6ISP, PR36ISP, and G-family forces.

\subsubsection{Spans for simple particles, components of root forces, and some objects}

We consider PR6ISP modeling.

We discuss spans for components of G-family root forces.

We start from the span of six that we posit for $4 \mathrm{G} 4$. (See discussion regarding equation (149).) We consider TA-side symmetries for G-family solutions. (See table 62.) We aim to develop numbers that belong in the table 62 column that has the label span (for $\iota_{I} \geq 6$ ). The number of generators of each 
Table 89: Cumulative features of various types of modeling

(a) Featured modeling

\begin{tabular}{|c|c|c|c|}
\hline Modeling & $\iota_{I}$ & New descriptions and new explanations & New subtleties \\
\hline $\begin{array}{l}\text { Ongoing } \\
\text { modeling }\end{array}$ & NR & - (Baseline) & - \\
\hline PR1ISP & 1 & $\begin{array}{l}\text { - New simple particles and root forces } \\
\text { - Baryon asymmetry } \\
\text { - Some dark matter }\end{array}$ & $\begin{array}{l}\text { - Dark energy negative } \\
\text { pressure } \\
\text { - Ratios of dark } \\
\text { energy density of the } \\
\text { universe to density of } \\
\text { the universe of } \\
\text { ordinary matter plus } \\
\text { dark matter }\end{array}$ \\
\hline PR6ISP & 6 & $\begin{array}{l}\text { - More dark matter } \\
\text { - Ratios of dark matter effects to } \\
\text { ordinary matter effects } \\
\text { - Objects, smaller than galaxies, that } \\
\text { feature dark matter }\end{array}$ & $\begin{array}{l}\text { - Spans } \\
\text { - Dark energy negative } \\
\text { pressure }\end{array}$ \\
\hline
\end{tabular}

(b) Possibly useful modeling

\begin{tabular}{llll}
\hline Modeling & $\iota_{I}$ & New descriptions and new explanations & New subtleties \\
\hline PR36ISP & 36 & - & $\bullet$ Ratios of dark \\
& & energy density of the \\
& universe to density of \\
& the universe of \\
& ordinary matter plus \\
& dark matter \\
\hline
\end{tabular}

Table 90: Relationships regarding PR1ISP, PR6ISP, PR36ISP, and G-family forces

Aspects

- Absent the notion that some components of G-family forces have spans of more than one, PR6ISP would correlate with six non-interacting sub-universes.

- In PR6ISP models, each sub-universe consists of an isomer of PR1ISP. The six isomers of PR1ISP might differ by as little as differing matches between generation of charged lepton and mass of charged lepton. (See discussion related to table 98.)

- In PR6ISP models, the main interactions between PR1ISP-like isomers correlate with the monopole component (or, 4G4) of gravity (or, 4G). Some other interactions between PR1ISP-like isomers correlate with a dipole (or, 4G48) component of gravity (or, 4G). Some other interactions between PR1ISP-like isomers correlate with dipole and quadrupole components of electromagnetism (or, 2G).

- Absent the notion that some components of G-family forces have spans of more than one, PR36ISP would correlate with 36 non-interacting sub-sub-universes.

- In PR36ISP models, each sub-sub-universe would consist of an isomer of PR1ISP.

- In PR36ISP models, six sub-universes pertain. Each sub-universe consists of a somewhat isomer of PR6ISP. Within each somewhat isomer of PR6ISP, the six PR1ISP-like isomers do not interact with each other via electromagnetism (or, $2 \mathrm{G}$ ).

- In PR36ISP models, the main interactions between somewhat-PR6ISP-like isomers correlate with dipole and quadrupole components of electromagnetism (or, $2 \mathrm{G}$ ). No interactions between somewhat-PR6ISP-like isomers correlate with gravity (or, 4G). Within each somewhat-PR6ISP-like isomer, the main interactions between PR1ISP-like isomers correlate with the monopole component (or, 4G4) of gravity (or, 4G). Within each somewhat-PR6ISP-like isomer, some other interactions between PR1ISP-like isomers correlate with a dipole (or, 4G48) component of gravity (or, 4G). (Per the previous item in this table, within each somewhat-PR6ISP-like isomer, no interactions between PR1ISP-like isomers correlate with electromagnetism.)

- In PR6ISP models and PR36ISP models, within each PR1ISP-like isomer, interactions correlating with gravity (or, 4G) occur and interactions correlating with electromagnetism (or, 2G) occur. 
Table 91: Aspects correlating with our positing that the span for each elementary particle - other than the G-family elementary particles - is one

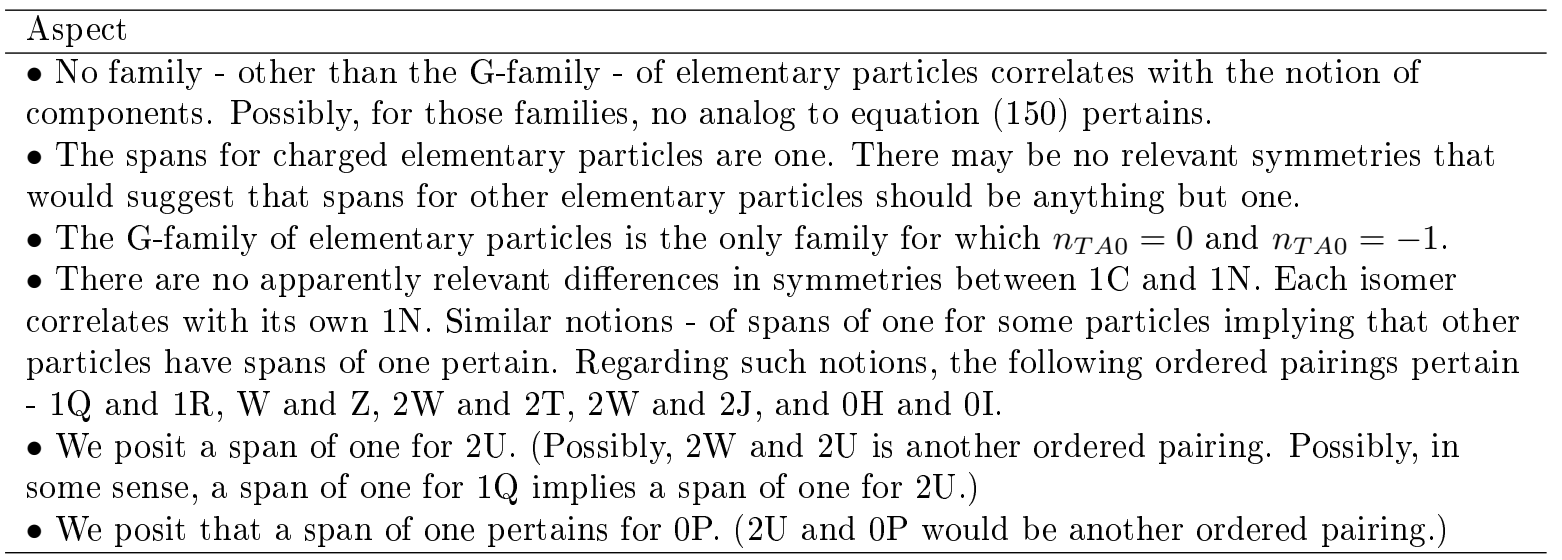

of $S U(3), S U(5)$, and $S U(7)$ divides evenly the integer 48, which is the number of generators of $S U(7)$. Regarding 4G4, we posit that the expression $6=g_{7} / g_{3}$ is relevant. (Regarding notation, see equation (46).) We generalize. We assert that, for each G-family solution for which a TA-side symmetry of $S U(j)$ pertains, equation (150) provides the span. We assume that we can generalize from the assumption that the span of $2 \mathrm{G} 2$ is one. For each G-family solution with no TA-side symmetry, the span is one.

$$
g_{7} / g_{j}
$$

Table 91 discusses aspects correlating with our positing that the span for each elementary particle other than the G-family elementary particles - is one.

The span for $1 \mathrm{Q} \otimes 2 \mathrm{U}$ is one, based on the non-zero charges of $1 \mathrm{Q}$ particles. We assume that the span for $1 \mathrm{R} \otimes 2 \mathrm{U}$ is one.

Equation (151) shows notation for denoting the span, s, for a simple particle or for a component of a root force.

$$
\Sigma(\mathrm{s}) \Phi \quad \text { or } \quad \Sigma(\mathrm{s}) \Phi \Gamma
$$

Table 92 shows the span for each component of G-family forces. The table pertains for each of PR6ISP modeling and PR36ISP modeling. Rows in table 92a list all $\Sigma \gamma$ components. Table 92a lists 2(6)G248 and does not list 2(1)G248. Rows in table 92b list $\Sigma \leq 8$ G-family force components that do not correlate with $\Sigma \gamma$. Table 92c lists some solutions that might - but do not necessarily - correlate with G-family force components. (See discussion related to equation (135).) For $\Sigma_{\max }=8$, table 92c is not physics-relevant. For $\Sigma_{\max }=20$, table $92 \mathrm{c}$ pertains regarding G-family forces.

We consider all three of PR1ISP modeling, PR6ISP modeling, and PR36ISP modeling.

Table 93 summarizes information regarding spans for simple particles, for hadron-like particles, and for some components of root forces. The table summarizes information regarding types of objects with which boson simple particles and some root force components interact. The table separates, based on a proposed modeling view, elementary particle Standard Model aspects from aspects that the elementary particle Standard Model does not embrace. The symbol $1 \mathrm{Q} \otimes 2 \mathrm{U}$ correlates with known and possible hadrons. The symbol $1 \mathrm{R} \otimes 2 \mathrm{U}$ correlates with possible hadron-like particles. Regarding the PR36ISP case, the notation $(\| 2 \mathrm{G})$ denotes a span that couples ordinary matter and doubly dark matter. The symbol $\| 2 \mathrm{G}$ correlates with the 3-element phrase parallel to 2G248. Regarding the PR36ISP case, the notation (\|4G) denotes a span that couples ordinary matter and dark matter. The symbol $\| 4 \mathrm{G}$ correlates with the 3-element phrase parallel to 4G4. Table 93a does not include G-family components that do not correlate with $\Sigma \gamma$ solutions. Regarding the PR6ISP case, the span for 2G68 is two. (See table 93b) Regarding the PR36ISP case, the span for $2 \mathrm{G} 68$ is two and the notion of $\| 2 \mathrm{G}$ pertains. Regarding the PR6ISP case, the pairings of isomers that isomers of $4 \mathrm{G} 48$ span might not equal the pairings of isomers that isomers of $2 \mathrm{G} 68$ span. The symbols : $4 \mathrm{G}$ and $: 2 \mathrm{G}$ correlate with this possible mismatch regarding pairings. Table 93 shows the extent to which each of the simple bosons and some of the root force components interacts directly with each of at least some simple fermions and with each of at least some multicomponent objects. The word Yes denotes that interactions occur. The symbol ${ }^{\dagger}$ denotes that rebuttable somewhat conservation of fermion generation pertains for $1 \mathrm{f}+1 \mathrm{~b} \rightarrow 1 \mathrm{f}+1 \mathrm{~b}$ interaction vertices. (Regarding the notation $1 \mathrm{f}+1 \mathrm{~b} \rightarrow 1 \mathrm{f}+1 \mathrm{~b}$, see 
Table 92: A catalog of components of G-family forces

(a) G-family force components for which $\Sigma \in \Gamma$

\begin{tabular}{cccccc}
\hline $\begin{array}{c}\Sigma \Gamma \\
(\Sigma \in \Gamma)\end{array}$ & $S$ & $\begin{array}{c}\text { Monopole } \\
\left(\mathrm{RSDF}=r^{-2}\right)\end{array}$ & $\begin{array}{c}\text { Dipole } \\
\left(\mathrm{RSDF}=r^{-3}\right)\end{array}$ & $\begin{array}{c}\text { Quadrupole } \\
\left(\mathrm{RSDF}=r^{-4}\right)\end{array}$ & $\begin{array}{c}\text { Octupole } \\
\left(\mathrm{RSDF}=r^{-5}\right)\end{array}$ \\
\hline Yes & 1 & $2(1) \mathrm{G} 2$ & $2(1) \mathrm{G} 24$ & $2(6) \mathrm{G} 248$ & \\
Yes & 2 & $4(6) \mathrm{G} 4$ & $4(2) \mathrm{G} 48$ & $4(1) \mathrm{G} 246$ & $4(1) \mathrm{G} 2468 \mathrm{a}$ \\
Yes & 2 & & & & $4(1) \mathrm{G} 2468 \mathrm{~b}$ \\
Yes & 3 & $6(2) \mathrm{G} 6$ & & & \\
Yes & 4 & $8(1) \mathrm{G} 8$ & & & $8(1) \mathrm{G} 2468 \mathrm{a} 468$ \\
Yes & 4 & & & $8(1) \mathrm{G} 2468 \mathrm{~b}$ \\
\hline
\end{tabular}

(b) G-family force components for which $\Sigma \notin \Gamma$ and $\Sigma \leq 8$

\begin{tabular}{|c|c|c|c|c|c|}
\hline $\begin{array}{c}\Sigma \Gamma \\
(\Sigma \in \Gamma)\end{array}$ & $S$ & $\begin{array}{c}\text { Monopole } \\
\left(\mathrm{RSDF}=r^{-2}\right)\end{array}$ & $\begin{array}{c}\text { Dipole } \\
\left(\mathrm{RSDF}=r^{-3}\right)\end{array}$ & $\begin{array}{c}\text { Quadrupole } \\
\left(\mathrm{RSDF}=r^{-4}\right)\end{array}$ & $\begin{array}{c}\text { Octupole } \\
\left(\mathrm{RSDF}=r^{-5}\right)\end{array}$ \\
\hline No & 1 & & $2(6) \mathrm{G} 46$ & $2(6) \mathrm{G} 468$ & \\
\hline No & 1 & & $2(2) \mathrm{G} 68$ & & \\
\hline No & 2 & & $4(6) \mathrm{G} 26$ & $4(6) \mathrm{G} 268$ & \\
\hline No & 3 & & $6(1) \mathrm{G} 24$ & $6(6) \mathrm{G} 248$ & \\
\hline No & 3 & & $6(2) \mathrm{G} 28$ & & \\
\hline No & 4 & & $8(6) \mathrm{G} 26$ & $8(1) \mathrm{G} 246$ & \\
\hline
\end{tabular}

(c) Some G-family solutions for which $\Sigma \geq 10$

\begin{tabular}{|c|c|c|c|c|c|}
\hline $\begin{array}{c}\Sigma \Gamma \\
(\Sigma \in \Gamma)\end{array}$ & $S$ & $\begin{array}{c}\text { Monopole } \\
\left(\mathrm{RSDF}=r^{-2}\right)\end{array}$ & $\begin{array}{c}\text { Dipole } \\
\left(\mathrm{RSDF}=r^{-3}\right)\end{array}$ & $\begin{array}{c}\text { Quadrupole } \\
\left(\mathrm{RSDF}=r^{-4}\right)\end{array}$ & $\begin{array}{c}\text { Octupole } \\
\left(\mathrm{RSDF}=r^{-5}\right)\end{array}$ \\
\hline No & 5 & & $10(2) \mathrm{G} 28$ & $10(6) \mathrm{G} 248$ & \\
\hline No & 5 & & $10(6) \mathrm{G} 46$ & $10(6) \mathrm{G} 468$ & \\
\hline No & 6 & & $12(2) \mathrm{G} 48$ & $12(1) \mathrm{G} 246$ & $12(1) \mathrm{G} 2468$ \\
\hline No & 6 & & & $12(6) \mathrm{G} 268$ & \\
\hline No & 7 & & 14(2)G68 & $14(6) \mathrm{G} 248$ & \\
\hline No & 8 & & & $16(6) \mathrm{G} 268$ & $16(1) \mathrm{G} 2468$ \\
\hline No & 9 & & & $18(6) \mathrm{G} 468$ & \\
\hline No & 10 & & & & $20(1) \mathrm{G} 2468$ \\
\hline
\end{tabular}


discussion regarding table 115.) The symbol ${ }^{\dagger \dagger}$ denotes that rebuttable always conservation of fermion generation pertains for $1 \mathrm{f}+1 \mathrm{~b} \rightarrow 1 \mathrm{f}+1 \mathrm{~b}$ interaction vertices. The word No denotes that interactions do not occur. Proposed modeling suggests the possibility that neither the $0 \mathrm{H}$ boson nor the $0 \mathrm{I}$ boson interacts directly with multicomponent objects. Proposed modeling suggests that G-family solutions for which the TA-side symmetry is $S U(5)$ or $S U(7)$ do not correlate with direct interactions with simple fermions. (See discussion related to table 59 and discussion related to table 62.) Tables $93 \mathrm{c}$ and $93 \mathrm{~d}$ summarize some concepts relevant to tables $93 \mathrm{a}$ and $93 \mathrm{~b}$.

Excitation of a boson encodes information specifying, in effect, the isomer or isomers that correlate with the excitation.

Table 94 summarizes aspects regarding information - centric to isomers - that proposed modeling fields carry. (Table 94 illustrates concepts that table 63 discusses.) In ongoing modeling, the electromagnetic field carries information that correlates with events that excited the field. Via de-excitations, people measure energies, momenta, and polarizations. People infer information about excitation events. (See discussion related to table 59.) Table 94 discusses additional information (compared to information that ongoing modeling fields carry) that proposed modeling fields carry.

We discuss concepts regarding the 2(2)G68 solution.

The 2(2)G68 solution does not belong to the set of $2 \gamma$ solutions and does not belong to the set of $\gamma 2$ solutions. The 2(2)G68 solution does not correlate with interactions with individual simple fermions. Table 84 correlates $\lambda=8$ with leptons and baryons. Each of table $66 \mathrm{~b}$ and discussion related to table 25 correlates $\lambda=8$ with rotation or spin. Table 58 correlates $\lambda=6$ with changes of internal states for multicomponent objects. We posit that 2(2) G68 correlates with some electromagnetic (or, $\Sigma=2$ ) interactions with atoms and other objects. We posit that those interactions include so-called hyperfine interactions.

Each of 2(1)G2 and 2(1)G24 correlates with some electromagnetic (or, $\Sigma=2$ ) interactions with atoms and other objects that include both baryons and leptons.

Unlike for the cases of electromagnetic interactions that correlate with 2(1)G2 and 2(1)G24, 2G produced by ordinary matter objects interacts with dark matter objects (for the case in which PR6ISP pertains to nature) or doubly dark matter objects (for the case in which PR36ISP pertains to nature) via 2(2)G68. Unlike for the cases of electromagnetic interactions that correlate with 2(1)G2 and 2(1)G24, $2 \mathrm{G}$ produced by some dark matter objects (for the case in which PR6ISP pertains to nature) or by some doubly dark matter objects (for the case in which PR36ISP pertains to nature) interacts with ordinary matter via 2(2)G68.

\subsubsection{Dark matter to ordinary matter ratios that modeling might predict or explain}

We discuss ratios that PR6ISP modeling or PR36ISP modeling might predict or explain.

Table 95 lists some approximate ratios of dark matter effects to ordinary matter effects that PR6ISP modeling might explain. We designed PR6ISP modeling to explain the five-plus to one ratios that people observe regarding densities of the universe. Here, the five correlates with dark matter isomers of charged (and other, except for G-family) elementary particles and the plus correlates with (ordinary matter isomer) hadron-like particles that do not interact with $2 \gamma$ force components. Galaxy clusters seem to be sufficiently large to comport with similar ratios. (However, galaxy clusters that are remnants of collisions of galaxy clusters might be exceptions. See discussion related to table 104.) Discussion just above regarding 2(2)G68 correlates with the approximately one to one ratio. (See, also, discussion related to equation (162).) Ratios of zero-plus to one, four to one, and one to zero-plus comport with roles of non-monopole gravity in scenarios regarding galaxy formation. (See discussion related to table 106.) The DMA:OMA ratios of zero-plus to one, the DMA:OMA ratio of four to one, and the DMA:OMA ratios of one to zero-plus comport with scenarios regarding some galaxies for which observations correlate with times well after galaxy formation. (See other discussion related to table 106.) Regarding the last row in table 95, see AX in table 106a and B0, B3, and BY in table 106b and note that only one of table 106a and table $106 \mathrm{~b}$ pertains to nature.

\subsubsection{Some properties of isomers of quarks and charged leptons}

We consider PR6ISP modeling and PR36ISP modeling.

Table 96 lists aspects that seem to correlate with each other regarding the one isomer that correlates with ordinary matter and the five isomers that correlate with dark matter. (See discussion - in table 20c - regarding SM6a. See discussion related to equation (108). See discussion related to table 98.)

We explore modeling that correlates each of the six relevant isomers with a range of $M^{\prime \prime}$. In equation (152), the integer $n$ numbers the isomers. Here, the ordinary matter isomer correlates with $n=0$. 
Table 93: Particles and solutions that correlate with one isomer and particles and solutions that might correlate with more than one isomer; plus, the extent to which simple bosons and some root force components interact with simple fermions and with multicomponent objects (with the symbol MCO denoting multicomponent objects; with the symbol ${ }^{\dagger}$ denoting that rebuttable somewhat conservation of fermion generation pertains; and with the symbol ${ }^{\dagger \dagger}$ denoting that rebuttable always conservation of fermion generation pertains)

(a) Particles and solutions, other than G-family components that are not $\Sigma \gamma$ components

\begin{tabular}{|c|c|c|c|c|c|c|}
\hline $\begin{array}{c}\text { Standard Model } \\
\text { entities }\end{array}$ & $\begin{array}{l}\text { Possible } \\
\text { entities }\end{array}$ & $\begin{array}{c}\text { PR1ISP } \\
\text { span }\end{array}$ & $\begin{array}{c}\text { PR6ISP } \\
\text { span }\end{array}$ & $\begin{array}{c}\text { PR36ISP } \\
\text { span }\end{array}$ & $\begin{array}{c}\text { 1b interact } \\
\mathrm{w} / 1 \mathrm{f}\end{array}$ & $\begin{array}{c}\text { 1b interact } \\
\mathrm{w} / \mathrm{MCO}\end{array}$ \\
\hline $0 \mathrm{H}$ & $0 \mathrm{I}$ & 1 & 1 & 1 & Yes $^{\dagger \dagger}$ & No \\
\hline- & $0 \mathrm{P}$ & 1 & 1 & 1 & No & Yes \\
\hline $1 \mathrm{C}$ & - & 1 & 1 & 1 & - & - \\
\hline $1 \mathrm{~N}$ & - & 1 & 1 & 1 & - & - \\
\hline $1 \mathrm{Q}$ & $1 \mathrm{R}$ & 1 & 1 & 1 & - & - \\
\hline $2 \mathrm{U}$ & - & 1 & 1 & 1 & $\mathrm{Yes}^{\dagger \dagger}$ & No \\
\hline $2 \mathrm{~W}$ & $2 \mathrm{~T}$ & 1 & 1 & 1 & $\mathrm{Yes}^{\dagger}$ & No \\
\hline- & $2 \mathrm{~J}$ & 1 & 1 & 1 & $\mathrm{Yes}^{\dagger}$ & Yes \\
\hline $1 \mathrm{Q} \otimes 2 \mathrm{U}$ & $1 \mathrm{R} \otimes 2 \mathrm{U}$ & 1 & 1 & 1 & - & - \\
\hline $2 \mathrm{G} 2$ & - & 1 & 1 & 1 & $\mathrm{Yes}^{\dagger \dagger}$ & Yes \\
\hline $2 \mathrm{G} 24$ & - & 1 & 1 & 1 & $\mathrm{Yes}^{\dagger \dagger}$ & Yes \\
\hline $2 \mathrm{G} 248$ & - & 1 & 6 & $6(\| 2 G)$ & $\mathrm{Yes}^{\dagger \dagger}$ & Yes \\
\hline- & $4 \mathrm{G} 4$ & 1 & 6 & $6(\| 4 \mathrm{G})$ & Yes $^{\dagger}$ & Yes \\
\hline - & $4 \mathrm{G} 48$ & 1 & $2(: 4 \mathrm{G})$ & $2(\| 4 \mathrm{G})$ & $\mathrm{Yes}^{\dagger}$ & Yes \\
\hline - & $4 \mathrm{G} 246$ & 1 & 1 & 1 & Yes $^{\dagger}$ & Yes \\
\hline - & $4 \mathrm{G} 2468 \mathrm{a}$ & 1 & 1 & 1 & Yes $^{\dagger}$ & Yes \\
\hline - & $4 \mathrm{G} 2468 \mathrm{~b}$ & 1 & 1 & 1 & Yes $^{\dagger}$ & Yes \\
\hline- & 6G6 & 1 & 1 & 1 & No & Yes \\
\hline - & 6G468 & 1 & 1 & 1 & Yes & Yes \\
\hline - & $8 \mathrm{G} 8$ & 1 & 1 & 1 & No & Yes \\
\hline - & $8 \mathrm{G} 2468 \mathrm{a}$ & 1 & 1 & 1 & Yes & Yes \\
\hline - & $8 \mathrm{G} 2468 \mathrm{~b}$ & 1 & 1 & 1 & Yes & Yes \\
\hline
\end{tabular}

(b) Selected G-family component that is not a $\Sigma \gamma$ component

\begin{tabular}{ccccccc}
\hline $\begin{array}{c}\text { Standard Model } \\
\text { entities }\end{array}$ & $\begin{array}{c}\text { Possible } \\
\text { entities }\end{array}$ & $\begin{array}{c}\text { PR1ISP } \\
\text { span }\end{array}$ & $\begin{array}{c}\text { PR6ISP } \\
\text { span }\end{array}$ & $\begin{array}{c}\text { PR36ISP } \\
\text { span }\end{array}$ & $\begin{array}{c}\text { 1b interact } \\
\text { w/ 1f }\end{array}$ & $\begin{array}{c}\text { 1b interact } \\
\text { w/ MCO }\end{array}$ \\
\hline- & $2 \mathrm{G} 68$ & 1 & $2(: 2 \mathrm{G})$ & $2(\| 2 \mathrm{G})$ & No & Yes \\
\hline
\end{tabular}

(c) Notes regarding the case PR6ISP

Note

- For one of $4 \mathrm{G} \Gamma_{4}$ with a span of two and $2 \mathrm{G} \Gamma_{2}$ with a span of two (and for a numbering system that numbers isomers using the integers zero through five), the pairings 0 -and-3, 1-and-4, and 2-and-5 might pertain. For the other one of the two $\left(4 \mathrm{G} \Gamma_{4}\right.$ and $\left.2 \mathrm{G} \Gamma_{2}\right)$, different pairings might pertain. (Note the notation $: 4 \mathrm{G}$ and $: 2 \mathrm{G}$.)

(d) Notes regarding the case PR36ISP

Note

- For each $\Sigma \Phi$ with $\Phi \neq \mathrm{G}$ and with a PR6ISP span of six or two, we assume that $\| 2 \mathrm{G}$ pertains.

- For each $4 \mathrm{G} \Gamma$ with a PR6ISP span of six or two, we assume that $\| 4 \mathrm{G}$ pertains. 
Table 94: Aspects regarding information - centric to isomers - that proposed modeling fields carry

\begin{tabular}{l}
\hline Aspect \\
- Compared to ongoing modeling models regarding fields, proposed modeling adds - to the set of \\
information that an excitation carries - information about the isomer or isomers correlating with \\
the excitation. \\
- For PR1ISP modeling, only one isomer pertains. The information correlates with a list of the one \\
isomer that correlates with creating the excitation. In effect, there is no added (compared to \\
ongoing modeling) information. \\
- For PR6ISP modeling, the information correlates with a list of the one, two, or six isomers that \\
correlate with creating the excitation. The number of isomers in the list equals the span for the \\
relevant particle or component. (See for example, table 93.) \\
- For PR36ISP modeling, the information correlates with a list of the one, two, or six isomers that \\
correlate with creating the excitation. The number of isomers in the list equals the span for the \\
relevant particle or component. (See, for example, table 93.) \\
- For each of PR1ISP modeling, PR6ISP modeling, and PR36ISP modeling, the excitation does \\
not necessarily carry information about the simple particles or force components that participated \\
in creating the excitation. \\
- For each of PR1ISP modeling, PR6ISP modeling, and PR36ISP modeling, de-excitation of an \\
excitation of a field must correlate with a set of isomers that includes an isomer that the \\
excitation-centric list includes.
\end{tabular}

Table 95: Approximate ratios of dark matter effects to ordinary matter effects (with DM denoting dark matter; with OM denoting ordinary matter; with A denoting amount; with OM CMB denoting cosmic microwave background radiation; and with * denoting that proposed modeling also suggests an explanation - correlating with PR36ISP modeling - that correlates with doubly-dark matter and does not correlate with dark matter)

\begin{tabular}{cl}
\hline $\begin{array}{c}\text { Approximate } \\
\text { DMA:OMA }\end{array}$ & Amounts \\
\hline $5^{+}: 1$ & Density of the universe \\
$5^{+}: 1$ & Amount of stuff in galaxy clusters \\
$1: 1$ or $1^{+}: 1$ & Amount of absorption of OM CMB via interactions with DM* atoms or OM \\
& atoms. \\
$0^{+}: 1$ & Amount of stuff in some early galaxies \\
$\approx 4: 1$ & Amount of stuff in some early galaxies \\
$1: 0^{+}$ & Amount of stuff in some early galaxies \\
$0^{+}: 1$ & Amount of stuff in some later galaxies \\
$\approx 4: 1$ & Amount of stuff in some later galaxies \\
$1: 0^{+}$ & Amount of stuff in some later galaxies \\
$\approx 3: 2$ to $\approx 4: 1$ & Amount of stuff in dark matter halo to amount of stuff near galaxy center (for \\
& some later galaxies) \\
\hline
\end{tabular}

Table 96: Aspects that seem to correlate with each other regarding the one isomer that correlates with ordinary matter and the five isomers that correlate with dark matter

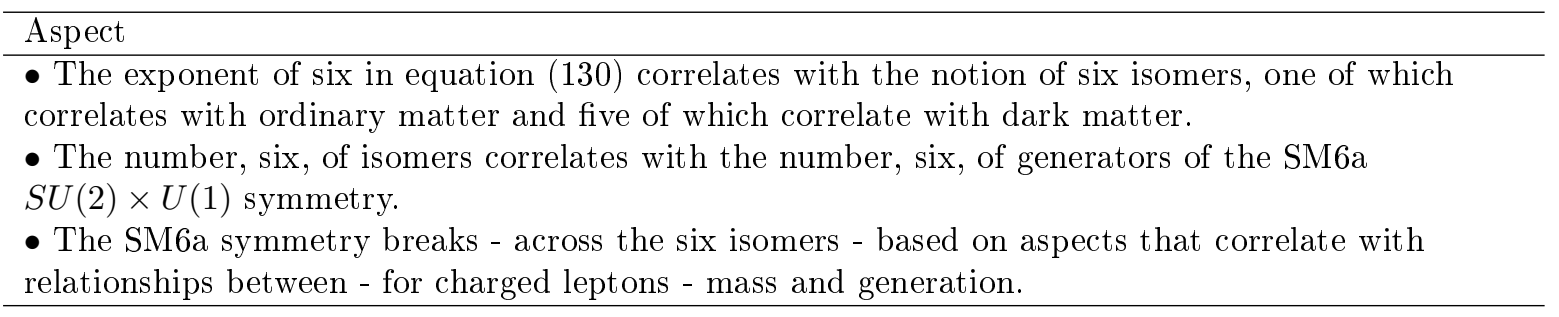


Table 97: Modeling pertaining to the one ordinary matter isomer and the five dark matter isomers

\begin{tabular}{ccc}
\hline$n$ & Formula & Interpretation \\
\hline $0,1,2,3,4$, or 5 & $M^{\prime \prime}=3 n+1$ & No particle \\
0 or 3 & $M^{\prime \prime}=3 n$ & Generation 1 \\
1 or 4 & $M^{\prime \prime}=3 n$ & Generation 3 \\
2 or 5 & $M^{\prime \prime}=3 n$ & Generation 2 \\
\hline
\end{tabular}

Table 98: Relationships between quark generation and lepton aspects

\begin{tabular}{ccccccc}
\hline$M^{\prime \prime}$ & Quark $n$ & $\begin{array}{c}\text { Quark } \\
\text { generation }\end{array}$ & $\begin{array}{c}\text { Lepton } n \\
\text { (for } n \text { even) }\end{array}$ & $\begin{array}{c}\text { Lepton } \\
\text { aspect }(\text { even } n)\end{array}$ & $\begin{array}{c}\text { Lepton } n \\
\text { (for } n \text { odd) }\end{array}$ & $\begin{array}{c}\text { Lepton } \\
\text { aspect }(\text { odd } n \text { ) }\end{array}$ \\
\hline 0 & 0 & 1 & 0 & - & - & - \\
1 & 0 & 2 & 0 & - & - & - \\
2 & 0 & 3 & 0 & 2 & 1 & - \\
3 & 1 & 1 & 0 & 3 & 1 & 3 \\
4 & 1 & 2 & - & - & 1 & - \\
5 & 1 & 3 & - & - & 1 & 1 \\
6 & 2 & 1 & 2 & 2 & - & 2 \\
7 & 2 & 2 & 2 & - & - & - \\
8 & 2 & 3 & 2 & 3 & 3 & - \\
9 & 3 & 1 & 2 & 1 & 3 & - \\
10 & 3 & 2 & - & - & 3 & 2 \\
11 & 3 & 3 & - & - & 3 & 3 \\
12 & 4 & 1 & 4 & 3 & - & - \\
13 & 4 & 2 & 4 & - & - & - \\
14 & 4 & 3 & 4 & 1 & 5 & 2 \\
15 & 5 & 1 & 4 & 2 & 5 & - \\
16 & 5 & 2 & - & - & 5 & 3 \\
17 & 5 & 3 & - & - & 5 & 1 \\
18 & - & - & - & - & & \\
\hline
\end{tabular}

$$
\text { isomer } n \leftrightarrow 3 n \leq M^{\prime \prime} \leq 3 n+3 \text {, for } 0 \leq n \leq 5
$$

Table 97 shows interpretations regarding modeling for the six isomers. (Compare with table 79.) Here, for $n \geq 1$, the $M^{\prime \prime}=3 n$ generation relevant to isomer $n$ equals the $M^{\prime \prime}=3(n-1)+3$ generation relevant to isomer $n-1$. Within an isomer, an overall result correlates with the same cyclic ordering, for generations, that table 79 shows.

We de-emphasize the following notions. Dark matter lepton masses might correlate with $m\left(M^{\prime \prime}, 3\right)$ and $M^{\prime \prime}>3$. Mathematics - such as for $M^{\prime \prime}<0$ - related to equation (108) might help estimate ongoing modeling values for neutrino masses. Results that correlate with $M^{\prime \prime}<0$ might be useful for estimating magnitudes of ordinary matter $2 \mathrm{G}$ interactions with dark matter analogs to ordinary matter charged leptons.

Table 98 shows, for each value of $n$, relationships between quark generation and lepton aspects. Table 98 extends table 97 and includes quarks. For each $n$, the order for quarks is generation one, generation two, and then generation three.

Table 98 has roots in models that correlate with the relative strengths of $2 \mathrm{G} 2$ and $4 \mathrm{G} 4$. We posit that aspects regarding mass correlate with the column with label $M^{\prime \prime}$. We posit that, for the lepton for which $n=1$ and $M^{\prime \prime}=3$, the generation is three and the mass equals the mass of the ordinary matter electron. This essay de-emphasizes the possibility that the masses of dark matter quarks correlate with changes of signs regarding the various $d^{\prime}\left({ }_{-}\right)$. (See equations (114), (115), and (116).) We assume that, for each of the six ranges that equation (152) specifies, the masses of the relevant quarks match the masses that table 79 would show if the masses in table 79 comported exactly with nature. For example, for each isomer, the mass of the generation-one quark with a magnitude of charge of $\left|q_{\epsilon}\right| / 3$ equals the mass of each other generation-one quark with a magnitude of charge of $\left|q_{\epsilon}\right| / 3$.

Work above might be appropriate for proposed modeling to be compatible with observations regarding the Bullet Cluster. (See discussion related to table 104.)

Table 99 summarizes two possible cases. 
Table 99: Two possible cases regarding the evolution of the five dark matter isomers and the one ordinary matter isomer

\begin{tabular}{ll}
\hline Case & Aspect \\
\hline Case S & $\begin{array}{l}\text { Isomer three evolves similarly to isomer zero. Isomer four evolves similarly to isomer } \\
\text { one. Isomer five evolves similarly to isomer two. Isomers one, two, four, and five do not } \\
\text { evolve similarly to isomer zero. }\end{array}$ \\
Case D & Each of the six isomers evolves differently from the other five isomers. \\
\hline
\end{tabular}

We correlate the two-word term case $\mathrm{S}$ with work above.

We do not know of data that correlates with the actual fractions of dark matter that pass through the collision with just gravitational interactions having significance. (See table 104.) Case S can comport with fractions that are less than or that somewhat exceed four-fifths (or, percentages that are less than or that somewhat exceed 80 percent).

Observations of more than 80-plus percent might correlate with geometric aspects such as the sizes and trajectories of the two galaxy clusters.

We explore the possibility that observations correlate with percentages that are too big for the combination of geometric aspects and case $\mathrm{S}$ to explain.

For percentages that are significantly above 80-plus percent, modeling might need to correlate with a significant difference in evolution between isomer three and isomer zero.

Proposed modeling allows for aspects that might comport with a significant difference in evolution between isomer three and isomer zero.

We correlate the two-word term case D with work below. (See table 99.)

One possibility correlates with at least one of the dominant charge or dominant handedness or dominant sign of 3LB for charged leptons. This possibility might correlate with a difference between even $n$ and odd $n$. (See table 98.) Aspects of this possibility might correlate with relationships between the $3 \mathrm{CH}$-centric $\pi_{@_{0}, @_{-1}}$ that correlates with oscillators SA1-and-SA2 and the 3LB-centric $\pi_{0}$, $@_{-1}$ that correlates with oscillators SA7-and-SA8. (See table 45.) For example, the following sentences might pertain. For even $n, @_{0}, @_{-1}, @_{0}$, and $@_{-1}$ pertain respectively for SA1, SA2, SA7, and SA8. For odd $n$, @ 0 , $@_{-1}, @_{-1}$, and $@_{0}$ pertain respectively for SA1, SA2, SA7, and SA8.

\subsubsection{Possible differences regarding the evolution of dark matter isomers}

We explore possible differences regarding the evolution of various dark matter isomers.

We explore case S. (See discussion related to table 98 and see table 99.)

We compare isomer one and isomer zero. (See discussion related to table 98.) For isomer one, the generation one charged lepton has a mass that is equal to the mass of an ordinary matter tauon. The isomer one generation one charged lepton has more mass than does the isomer zero generation one lepton (which is the electron). The isomer one generation two charged lepton has a mass that is equal to the mass of the isomer zero electron. The isomer one generation three charged lepton has a mass that is equal to the mass of the isomer zero muon. Regarding isomer one, each one of the generation two and generation three charged leptons has a mass that is less than the mass of the respective isomer zero charged lepton.

We discuss times for which the density and temperature suffice to catalyze tweak-based interactions that do not conserve fermion generation. Regarding generation one quarks, more transitions to higher generations of leptons occur for isomer one than for isomer zero. (For isomer one, the higher generations of leptons are less massive than the generation one lepton. Also, the isomer one generation one charged lepton is more massive than the isomer zero generation one charged lepton.) Regarding generation two and generation three quarks, fewer transitions - per unit time - to lower generations of leptons occur for isomer one than for isomer zero.

The formation of hadron-like particles based on generation one quarks occurs later for isomer one than for isomer zero.

Isomer one phenomena such as star formation and nuclear fusion start later and at lower densities of atoms than do similar ordinary matter (or, isomer zero) phenomena. Similar results - of later start and lower densities, compared to the ordinary matter isomer - pertain for isomers two, four, and five. (Details regarding isomer four have similarities to details regarding isomer one. Details regarding isomer five have similarities to details regarding isomer two. Details regarding isomer two and five differ from details regarding isomers one and four.) Details regarding isomer three have similarities to details regarding isomer zero. 
Table 100: Ordinary matter, four cool dark matter isomers, and the one other dark matter isomer (with results correlating with case $\mathrm{S}$, as per table 99)

\begin{tabular}{cl}
\hline Isomers $(n)$ & Aspect - regarding each isomer \\
\hline 0 & Is ordinary matter. \\
3 & Evolves similarly to ordinary matter. \\
$1,2,4$, and 5 & Evolves into cool dark matter. \\
\hline
\end{tabular}

Table 101: Ordinary matter and five cool dark matter isomers (with results possibly correlating with case D, as per table 99)

\begin{tabular}{cl}
\hline Isomers $(n)$ & Aspect - regarding each isomer \\
\hline 0 & Is ordinary matter. \\
$1,2,3,4$, and 5 & Evolves into cool dark matter. \\
\hline
\end{tabular}

Each one of the four isomers that exhibit reduced star formation and reduced fusion somewhat rapidly features mainly non-zero mass objects and dark matter photons. From that time forward, the dominant effects are clumping of the objects and cooling of the dark matter photons. The clumping has bases in $4 \mathrm{G}$ interactions. We correlate the three-word term cool dark matter with this dark matter state of mainly non-zero mass objects and dark matter photons. (We do so to denote scientific similarity to, but not necessarily linguistic equality with, ongoing modeling uses of the three-word term cold dark matter.)

Table 100 pertains regarding case S. (See table 99.)

We anticipate that notions that table 100 summarizes might explain aspects of the Bullet Cluster and aspects regarding galaxy dark matter halos. (Regarding the Bullet Cluster, see discussion related to table 104. Regarding dark matter halos, see, for example, discussion related to table 106.)

Table (101) might pertain regarding case D. (See table 99.) The form of cool dark matter correlating with isomer three might differ considerably from the forms of cool dark matter correlating with isomers one and four and with isomers two and five.

To the extent that notions that table 100 summarizes do not explain aspects of the Bullet Cluster and aspects regarding galaxy dark matter halos, notions that table 101 summarizes might explain aspects of the Bullet Cluster and aspects regarding galaxy dark matter halos.

Work elsewhere in this essay assumes that case $\mathrm{S}$ pertains and that one can de-emphasize case D. (See, for example, discussion related to table 104 and discussion related to table 106.) We think that, an ability for case $\mathrm{S}$ to comport with observations - for at least other than the Bullet Cluster and possibly for the Bullet Cluster - might suffice for proposed modeling to de-emphasize case D.

\subsection{Predictions and explanations regarding astrophysics and cosmology}

We explore aspects of astrophysics and cosmology.

\subsubsection{A specification for dark matter and ordinary matter}

We summarize a combined description of dark matter and ordinary matter. (See, for example, table 93a.) This description correlates with PR6ISP modeling.

PR6ISP modeling correlates with six isomers of particles that interact via $2 \mathrm{G} 2$. Here, each of the six isomers of particles correlates with its own isomer of $2 \mathrm{G} 2$. Each of the six isomers correlates with its own isomer of $1 \mathrm{R} \otimes 2 \mathrm{U}$ hadron-like particles. The $1 \mathrm{R} \otimes 2 \mathrm{U}$ hadron-like particles correlating with each - including the ordinary matter isomer - of the six isomers measure as being dark matter. Otherwise, from the perspective of observations that people make, one isomer (of non-G-family elementary particles) measures as being ordinary matter and the other five isomers (of non-G-family elementary particles) measure as being dark matter.

Regarding each ordinary matter simple particle, each one of the five dark matter isomers includes a simple particle that has the same spin, the same magnitude of charge, and the same mass. If the ordinary matter simple particle is a charged lepton, for each of four of the five dark matter isomers the respective same-spin, same-magnitude-of-charge, and same-mass charged lepton correlates with a generation number that differs from the generation number that pertains for the ordinary matter charged lepton.

For each one of those four dark matter isomers, evolution regarding objects differs from the evolution regarding ordinary matter objects. Those four isomers evolve into cool dark matter. (See table 100.) 


\subsubsection{Densities of the universe}

Ongoing modeling discusses five partial densities of the universe. The symbol $\Omega_{\nu}$ denotes neutrino density of the universe. The symbol $\Omega_{\mathrm{c}}$ denotes dark matter (or, cold dark matter) density of the universe. The symbol $\Omega_{\mathrm{b}}$ denotes ordinary matter (or, baryonic matter) density of the universe. The symbol $\Omega_{\gamma}$ denotes photon density of the universe. The symbol $\Omega_{\Lambda}$ denotes dark energy density of the universe. Each of the five densities correlates with data. Equation (153) pertains regarding the total density of the universe, $\Omega$.

$$
\Omega=\Omega_{\nu}+\Omega_{\mathrm{c}}+\Omega_{\mathrm{b}}+\Omega_{\gamma}+\Omega_{\Lambda}
$$

In ongoing modeling, the symbol $\Omega_{\mathrm{c}}$ correlates with all dark matter. To the extent that proposed modeling PR6ISP modeling or PR36ISP modeling comports with nature, the symbol $\Omega_{\mathrm{c}}$ correlates with all of the three aspects - $1 \mathrm{R} \otimes 2 \mathrm{U}$ hadron-like particles, the four dark matter isomers that we correlate above with the word cool, and the one dark matter isomer that we do not necessarily correlate above with the word cool - that proposed modeling correlates with the term dark matter.

Proposed modeling suggests equation 154 . The symbol $\Omega_{1 \mathrm{R} 2 \mathrm{U}, 0}$ denotes the density of the universe that correlates with $1 \mathrm{R} \otimes 2 \mathrm{U}$ that correlates with the ordinary matter isomer (or, isomer zero). The symbol $\Omega_{\mathrm{b},>0}$ denotes the baryonic density of the universe that correlates with the five dark matter isomers (or, isomers one through five). The symbol $\Omega_{\gamma,>0}$ denotes the photon density of the universe that correlates with the five dark matter isomers (or, isomers one through five). The symbol $\Omega_{\nu,>0}$ denotes the neutrino density of the universe that correlates with the five dark matter isomers (or, isomers one through five).

$$
\Omega_{\mathrm{c}}=\Omega_{1 \mathrm{R} 2 \mathrm{U}, 0}+\Omega_{\mathrm{b},>0}+\Omega_{\gamma,>0}+\Omega_{\nu,>0}
$$

We interpret data regarding recent states of (ordinary matter) CMB (or, cosmic microwave background radiation) as correlating with equation $(155)$. The symbol $\Omega_{1 \mathrm{R} 2 \mathrm{U}, 0}$ correlates with the plus in the ratio five-plus to one. The relationships $\Omega_{\mathrm{b}} \gg \Omega_{\gamma}$ and $\Omega_{\mathrm{b}} \gg \Omega_{\nu}$ pertain regarding data. (Reference [13] provides data regarding $\Omega_{\mathrm{b}} \gg \Omega_{\gamma}$ and $\Omega_{\mathrm{b}} \gg \Omega_{\nu}$.) Each of isomers one, two, ..., and six has its own $1 \mathrm{R} \otimes 2 \mathrm{U}$, Equation 156 pertains.

$$
\begin{gathered}
\Omega_{\mathrm{b},>0} \approx \Omega_{\mathrm{b},>0}+\Omega_{\gamma,>0}+\Omega_{\nu,>0} \approx 5\left(\Omega_{\mathrm{b}}+\Omega_{\gamma}+\Omega_{\nu}\right) \approx 5 \Omega_{\mathrm{b}} \\
\Omega_{1 \mathrm{R} 2 \mathrm{U}, 0} \approx\left(\Omega_{\mathrm{c}}-5 \Omega_{\mathrm{b}}\right) / 6
\end{gathered}
$$

Equation 160 estimates $\Omega_{1 \mathrm{R} 2 \mathrm{U}, 0}$ for the current state of the universe. (Reference [13] provides the data that equations (157), (158), and (159) show.)

$$
\begin{gathered}
\Omega_{\mathrm{b}} \approx 0.0484 \pm 0.001 \\
\Omega_{\mathrm{c}} \approx 0.258 \pm 0.011 \\
\Omega_{\gamma} \approx 0.0000538 \pm 0.0000150 \\
\Omega_{1 \mathrm{R} 2 \mathrm{U}, 0} \approx 0.0027
\end{gathered}
$$

Reasons exist for not taking results - that equation (160) shows - to be exact. For example, we note the size of the standard deviation in equation (158).

\subsection{3. $D M$ to $O M$ density of the universe ratios inferred from data regarding $C M B$}

People use data from observations of CMB (or, cosmic microwave background radiation) to infer ratios of dark matter density of the universe to ordinary matter density of the universe. (See discussion that leads to table 89 and includes equation (150).) A ratio of five-plus to one seems to pertain for billions of years. (See discussion related to equation (155) and discussion related to equation (161).) We use that ratio to posit the six-isomer basis for PR6ISP modeling. 


\subsubsection{Constancy of actual density of the universe ratios re DM and $O M$}

We discuss modeling regarding the ratio of actual dark matter density of the universe to actual ordinary matter density of the universe.

Elsewhere, we discuss possible threshold energies pertaining to reactions that might produce $1 \mathrm{R} \otimes 2 \mathrm{U}$ hadron-like particles. (See, for example, discussion regarding equations (206) and (207).) For each of the six isomers, the relative densities of the universe of $1 \mathrm{R} \otimes 2 \mathrm{U}$ hadron-like particles and ordinary matter $1 \mathrm{Q} \otimes 2 \mathrm{U}$ hadron particles might be essentially constant after the universe cools to a temperature correlating with an energy of $81 \mathrm{GeV}$. (See discussion regarding equations (206) and (207).) Regarding PR6ISP modeling and PR36ISP modeling, proposed modeling does not necessarily include interactions that would convert ordinary matter $1 \mathrm{Q} \otimes 2 \mathrm{U}$ to dark matter $1 \mathrm{Q} \otimes 2 \mathrm{U}$ or interactions that would convert dark matter $1 \mathrm{Q} \otimes 2 \mathrm{U}$ to ordinary matter $1 \mathrm{Q} \otimes 2 \mathrm{U}$.

The actual ratio of dark matter density of the universe to ordinary matter density of the universe might not much change after the cooling to the temperature correlating with the energy $81 \mathrm{GeV}$. That energy correlates with a temperature of about $10^{15}$ degrees Kelvin. That temperature correlates with a time that is less than $10^{-4}$ seconds after the Big Bang. (Reference [23] notes that a temperature of $10^{13}$ degrees Kelvin correlates with a time of $10^{-4}$ seconds after the Big Bang.)

Measured ratios of dark matter density of the universe to ordinary matter density of the universe would not much change regarding times for which equation (161) pertains. (Perhaps, see equations (157) and (159).) That time range starts somewhat after 380,000 years after the Big Bang and continues through now.

$$
\Omega_{\gamma} \ll \Omega_{\mathrm{b}} \text { and } \Omega_{\nu} \ll \Omega_{\mathrm{b}}
$$

\subsubsection{A possibly DM effects to OM effects ratio inferred from data regarding $C M B$}

People measure specific depletion of $\mathrm{CMB}$ and attribute some of that depletion to hyperfine interactions with (ordinary matter) hydrogen atoms. (See reference [24.) The amount of depletion is twice or somewhat more than twice the amount that people expected. At least one person speculates that the amount above expectations correlates with effects of dark matter. (See reference [25.)

Proposed modeling suggests the following explanation. Solution 2(2)G68 has a span of two. 2(2)G68 interactions are 2(2)G interactions. Equation (162) pertains. Solution 2(2)G68 does not correlate with interactions with individual simple fermions. (The TA-side symmetry is $S U(5)$. See table 62 and table 93b.) Solution 2(2)G68 might correlate with hyperfine interactions. (Note, for example, that the six in $\Gamma$ might correlate with aspects of multicomponent objects. The eight in $\Gamma$ might correlate with at least one of lepton number and spin.) Half or somewhat less than half of the observed absorption correlates with the ordinary matter isomer of hydrogen atoms. An equal amount of the observed effect correlates with hydrogen-atom isomers that correlate with one dark matter isomer or with one doubly dark matter isomer. The dark matter case correlates with PR6ISP modeling. The doubly dark matter case correlates with PR36ISP modeling.

$$
2 \mathrm{G} 68 \notin 2 \gamma, 2 \mathrm{G} 68 \notin \gamma 2
$$

To the extent that the absorption by ordinary matter is less than half of the total absorption, the following explanations might pertain. One explanation correlates with the notion that the evolution of the relevant non-ordinary-matter isomer might differ from the evolution of the ordinary matter isomer. The non-ordinary-matter isomer might have more hydrogen-atom-like objects than does the ordinary matter isomer. One explanation correlates with $2 \mathrm{G} \Gamma$ solutions with spans of at least two. Each one of solutions 2(6)G46 and 2(6)G468 might pertain. The number six appears in both the $\Gamma$ for 2(2)G46 and the $\Gamma$ for 2(6)G468. Solution 2(2)G46 correlates with a dipole effect. Solution 2(6)G468 correlates with a quadrupole effect.

Proposed modeling might contribute to credibility for assumptions and calculations that led to the prediction for the amount of depletion that correlates with ordinary matter hydrogen atoms. (Regarding the assumptions and calculations, see reference [26].)

\subsubsection{The rate of expansion of the universe}

Two thought experiments set the stage for discussing aspects regarding the rate of expansion of the universe.

We consider one thought experiment. We consider two similar neighboring clumps of stuff. We assume that the clumps are moving away from each other. We assume that the clumps will continue to move away from each other. We assume that, initially, interactions correlating with RSDF $r^{-(n+1)}$ dominate 
Table 102: Eras and components of $4 \mathrm{G}$ forces, regarding expansion of the universe

\begin{tabular}{cccccc}
\hline Era & A/R & RSDF & $\begin{array}{c}\text { Components } \\
\text { of } 4 \gamma\end{array}$ & $\begin{array}{c}\text { Other } \\
\text { components } \\
\text { of } 4 \mathrm{G}\end{array}$ & $\begin{array}{c}\text { Span } \\
\text { (PR6ISP } \\
\text { or } \\
\text { PR36ISP) }\end{array}$ \\
\hline early acceleration & net repulsive & $r^{-5}$ & $4(1) \mathrm{G} 2468 \mathrm{a}$, & & 1 \\
deceleration & net attractive & $r^{-4}$ & $4(1) \mathrm{G} 2468 \mathrm{~b}$ & & \\
$\begin{array}{c}\text { recent acceleration } \\
\text { (recent, for smaller } \\
\text { objects) }\end{array}$ & net repulsive & $r^{-3}$ & $4(2) \mathrm{G} 48$ & $4(2) \mathrm{G} 26$ & 2 \\
\hline
\end{tabular}

regarding interactions between the two clumps. We assume that the two clumps interact via interactions correlating with RSDF $r^{-n}$. We assume that no other forces have adequate relevance. We assume that the distance between the objects increases adequately. Eventually, the RSDF $r^{-n}$ force dominates the RSDF $r^{-(n+1)}$ force.

We consider a similar thought experiment. We consider two similar neighboring clumps. We assume that these clumps are less interactive (for example, less massive) than the two clumps in the first thought experiment. Generally, dominance of the RSDF $r^{-n}$ force over the RSDF $r^{-(n+1)}$ force occurs sooner for the two clumps in the second thought experiment than it does for the two clumps in the first thought experiment.

Table 102 summarizes, regarding the rate of expansion of the universe, eras and $4 \mathrm{G}$ force components. In this context, the eras pertain to the largest objects that people can directly infer. Early acceleration pertains for some time after the Big Bang. Then, deceleration pertains for some billions of years. Acceleration pertains for the most recent some billions of years. (Regarding observations that correlate with the eras that correlate with deceleration and recent acceleration, see references [27, [28, [29, and [30].) Regarding smaller objects, dominant forces within objects and between neighboring objects have, at least conceptually, generally transited parallels to the above-mentioned eras and now generally exhibit behavior correlating with RSDF of $r^{-2}$. (Discussion regarding table 119 notes that high-outflow phenomena related to black holes or neutron stars might provide exceptions regarding the notion of complete dominance correlating with an RSDF of $r^{-2}$. For some aspects of these cases, $r^{-3}$ net repulsion might pertain.) In table 102, the column labeled A/R notes net effects, across force components dominating for each era. The column labeled components of $4 \gamma$ lists solutions that might correlate with significant forces. (See table 65. Proposed modeling suggests that, for the purposes of this discussion, neither 4(1)G268 nor 4(2)G26 correlates with significant effects.) Proposed modeling suggests (but does not necessarily require) that, for the components of $4 \gamma$ that table 102 lists, the two-word term net repulsive correlates with a notion of essentially always repulsive (though perhaps sometimes not significantly repulsive). Proposed modeling suggests (but does not necessarily require) that, for the components of $4 \gamma$ that table 102 lists, the two-word term net attractive correlates with a notion of essentially always attractive (though perhaps sometimes not significantly attractive).

Proposed modeling suggests that the ongoing modeling notion of dark energy negative pressure correlates with the 4(2)G48 component (and possibly with the 4(1)G2468a and 4(1)G2468b components) of $4 \gamma$.

A better characterization than the six-word term rate of expansion of the universe might feature a notion of the rates of moving apart of observed very large astrophysical objects.

\subsubsection{Phenomena - including the creation of isomers - before through just after inflation}

Ongoing modeling suggests that an inflationary epoch might have occurred. Ongoing modeling suggests that the epoch started around $10^{-36}$ seconds after the Big Bang. (This essay de-emphasizes discussing aspects that might correlate with a time that people correlate with the two-word term Big Bang.) Ongoing modeling suggests that the epoch ended around $10^{-33}$ seconds to $10^{-32}$ seconds after the Big Bang. We are not certain as to the extent to which data confirms the occurrence of an inflationary epoch.

Ongoing modeling includes models that people claim would support notions of inflation. The models point to states of the universe, at and somewhat after the inflationary epoch, that would provide bases for evolution that would be consistent with observations about later phenomena and would be consistent with aspects of ongoing modeling. (Reference [31] summarizes aspects related to inflation, points to references regarding ongoing modeling, and discusses some ongoing modeling work.) 
Table 103: Some possible reactions involving pairs of jay bosons

\begin{tabular}{ccc}
\hline $\begin{array}{c}\text { Incoming } \\
\text { particles }\end{array}$ & $\begin{array}{c}\text { Effective } \\
\text { refraction } \\
\left(n_{S A 0}\right)\end{array}$ & $\begin{array}{c}\text { Outgoing } \\
\text { particles }\end{array}$ \\
\hline $2 \mathbf{J}_{ \pm}+2 \mathbf{J}_{ \pm}$ & $0^{-}$ & $4 \mathrm{G}+0 \mathrm{I}, 2 \mathrm{G}+0 \mathrm{I}, 0 \mathrm{I}+0 \mathrm{I}$ \\
$2 \mathrm{~J}_{ \pm}+2 \mathbf{J}_{\mp}$ & $0^{-}$ & $2 \mathrm{G}+0 \mathrm{I}, 0 \mathrm{I}+0 \mathrm{I}$ \\
$2 \mathbf{J}_{0}+2 \mathbf{J}_{0}$ & $0^{-}$ & $4 \mathrm{G}+0 \mathrm{I}, 2 \mathrm{G}+0 \mathrm{I}, 0 \mathrm{I}+0 \mathrm{I}$ \\
$2 \mathrm{~J}_{ \pm}+2 \mathbf{J}_{ \pm}$ & $(-1)^{+}$ & $4 \mathrm{G}+0 \mathrm{I}$ \\
$2 \mathbf{J}_{ \pm}+2 \mathbf{J}_{\mp}$ & $(-1)^{+}$ & $0 \mathrm{I}+0 \mathrm{I}$ \\
$2 \mathbf{J}_{0}+2 \mathbf{J}_{0}$ & $(-1)^{+}$ & $4 \mathrm{G}+0 \mathrm{I}, 0 \mathrm{I}+0 \mathrm{I}$ \\
\hline
\end{tabular}

Reference [32] suggests the possibility that a repulsive aspect of gravity drove phenomena correlating with the inflationary epoch. The reference suggests that the composition of the universe was nearly uniform spatially. The reference suggests the importance of a so-called inflaton field.

We anticipate discussing phenomena that might correlate with times during and just after the inflationary epoch.

We speculate about phenomena that might have occurred before the inflationary epoch.

Table 103 shows some possible reactions involving pairs of jay bosons. The leftmost column describes the pair of incoming jay bosons. The middle column describes two possibilities regarding modeling. (See discussion related to table 68.) For one possibility, an effective $n_{S A 0}$ correlates with $0^{-}$. For the other possibility, an effective $n_{S A 0}$ correlates with $(-1)^{+}$. The rightmost column shows possibilities for pairs of outgoing particles. (Table 103 correlates with the case $\mathrm{n}=2$ in table 115 .) For $n_{S A 0} \lesssim 0$, each of the three jay bosons might exhibit aspects of longitudinal polarization. Each of the $2 \mathbf{J}_{+}$and $2 \mathbf{J}_{-}$would exhibit two states. The $2 \mathrm{~J}_{0}$ would exhibit three states. For $n_{S A 0} \gtrsim-1$, each of the three jay bosons would not exhibit aspects of longitudinal polarization. Each of the $2 \mathbf{J}_{+}$and $2 \mathbf{J}_{-}$would exhibit one state. The $2 \mathbf{J}_{0}$ would exhibit two states. We discuss, as an example, the case of incoming $2 \mathrm{~J}_{ \pm}+2 \mathrm{~J}_{\mp}$. For $n_{S A 0} \gtrsim-1$, the incoming particles correlate with units of spin that have opposite circular polarizations. In effect, the circular polarizations sum to zero circular polarization. The outgoing pair 0I $+0 \mathrm{I}$ is appropriate. For $n_{S A 0} \lesssim 0$, one of the incoming particles might have a unit of circular polarization and the other incoming particle might not have circular polarization. Producing $2 \mathrm{G}+0 \mathrm{I}$ is possible.

We explore the notion that, just before the inflationary epoch, the main component of the universe might have consisted of jay bosons. (One might speculatively correlate the notion of a universe composed essentially only of jay bosons with seemingly unique aspects of modeling regarding the $0 \mathrm{G} \emptyset$ solution.)

Proposed modeling correlates (absence effects that would correlate with notions of refraction) the expression $n_{S A 0}=-1$ with jay bosons. Ongoing modeling seems to suggest that nature creates photons (or, $2 \mathrm{G}$ ) primarily after the inflationary epoch. Regarding time just before inflation, we assume that the lower half of table 103 pertains and that the upper half of table 103 does not pertain.

We assume that the particle density is sufficiently large that modeling can correlate the production of $4 \mathrm{G}$ with the $4 \mathrm{G} 2468 \mathrm{x}$ components of $4 \mathrm{G}$. (Regarding the symbol $4 \mathrm{G} 2468 \mathrm{x}$, see table 82 .)

Equation (163) describes a possible interaction. The span for each of $2 \mathrm{~J}_{-}, 4 \mathrm{G} 2468 \mathrm{x}$ and $0 \mathrm{I}$ is one. For PR6ISP models, the one six-fold (or, $S U(2) \times U(1))$ relevant symmetry could correlate with equal creation of six isomers for each of 2.J-, 4G2468x, and 0I. (See tables 21 and 58.) For PR36ISP models, the two six-fold (or, $S U(2) \times U(1)$ ) relevant symmetries might correlate with equal creation of 36 isomers for each of $2 \mathrm{~J}_{-}, 4 \mathrm{G} 2468 \mathrm{x}$, and $0 \mathrm{I}$.

$$
2(1) \mathbf{J}_{-}+2(1) \mathbf{J}_{-} \rightarrow 4(1) \mathrm{G} 2468 \mathrm{x}+0(1) \mathrm{I}
$$

We turn our attention to the inflationary epoch.

Proposed modeling suggests the possibility that, during the inflationary epoch, aye particles (or, 0I particles) provided a major non-root-force component of the universe. The aye particle matches ongoing modeling notions of a boson with zero spin. (See reference [31].) Ongoing modeling uses the word inflaton to name that boson. Proposed modeling suggests the possibility that the octupole components of $4 \gamma$ provided the repulsive aspect of gravity. (See, for example, table 119.) Those components interact with individual simple particles and are repulsive. Equation (164) shows such an interaction. Here, $\mathrm{x}$ and $y$ might be either of $\mathrm{a}$ and $\mathrm{b}$.

$$
0(1) \mathrm{I}+4(1) \mathrm{G} 2468 \mathrm{x} \rightarrow 0(1) \mathrm{I}+4(1) \mathrm{G} 2468 \mathrm{y}
$$


Equation 165 shows a phenomenon, which might pertain to the extent that aye and jay bosons co-existed in a dense environment.

$$
2(1) \mathbf{J}_{0}+2(1) \mathbf{J}_{0}+0(1) \mathrm{I} \rightarrow 2(1) \mathbf{J}_{0}+2(1) \mathbf{J}_{0}+8(1) G 2468 x
$$

The end of the inflationary epoch might correlate with a change, regarding effects of $4 \mathrm{G}$, from octupole repulsion being dominant to quadrupole attraction being dominant. (This essay does not speculate regarding the extent to which jay bosons continued to have significant effects - except, for example, effects that ongoing modeling correlates with the Pauli exclusion principle - after the inflationary epoch. Possibly, the density of stuff - other than jay bosons - decreased enough that - in a sense of ongoing modeling - essentially no non-virtual jay bosons existed.) The end of the inflationary epoch might also correlate with a growth of spatial inhomogeneities regarding (at least) aye particles. The quadrupole component of $4 \gamma$ might help catalyze some of the spatial inhomogeneities. The quadrupole component of $4 \gamma$ might amplify some of the spatial inhomogeneities.

We turn our attention to just after the inflationary epoch.

References [32] and [31] suggest that inflaton particles dominated (what proposed modeling might characterize as) the non-root-force composition of the universe for some time after the inflationary epoch. Inflatons produced a cascade of interactions that led to a preponderance of protons, neutrons, and electrons. Clumping of the resulting hydrogen atoms led to the formation of stars.

Proposed modeling suggests the possibility that, for some time just after the inflationary epoch, the aye particle might have been a dominant non-root-force component of the universe. The dominant Gfamily force component would have been the quadrupole component of $4 \gamma$. That component interacts with individual simple particles and is attractive. Interactions between aye particles would produce components of $2 \mathrm{G}$ forces. (See equation (166).) Each of proposed modeling and ongoing modeling includes interactions by which $2 \mathrm{G}$ components produce matter-and-antimatter pairs of simple fermions. Interactions between elementary particles would produce simple bosons that are not ayes or jays. (This essay does not speculate regarding the extent to which aye bosons continued to have significant effects much after the inflationary epoch. Possibly, the density of stuff - other than aye bosons - decreased enough that - in a sense of ongoing modeling - essentially no non-virtual aye bosons existed.) From there, the above-mentioned cascade could occur. Proposed modeling suggests the possibility that attraction based on the quadrupole component of $4 \gamma$ contributed to clumping.

$$
0 \mathrm{I}+0 \mathrm{I} \rightarrow 2 \mathrm{G}+2 \mathrm{G}
$$

Discussion above de-emphasizes the question of the extent to which, for clumps or objects that involve multiple simple particles, $4 \gamma$ octupole repulsion might dominate $4 \gamma$ quadruple attraction for at least some time after the end of the inflationary epoch.

We discuss isomers and spans.

Our work considers three $\mathrm{PR} \iota_{I}$ ISP cases $-\iota_{I}$ is one, $\iota_{I}$ is six, and $\iota_{I}$ is 36 . Table 93 suggests that the span for each of the $2 \mathrm{~J}$ bosons, $0 \mathrm{I}$ boson, quadrupole component of $4 \gamma$, and the two octupole components of $4 \gamma$ is one.

We explore the case for which $\iota_{I}$ is six.

As soon as $4 \mathrm{G}$ excites - for example, via the interaction that equation (163) symbolizes - isomers can couple to each other. We consider an excitement of $4 \mathrm{G}$ that - for discussion purposes - we correlate with equation (163). The excitement can interact with any one of the six isomers of 2J. The excitement can interact with any one of the six isomers of 0I. Such (in effect, monopole and also - via 4(2)G48 - dipole) coupling has impact, even though - from a standpoint of kinematics or dynamics - octupole components play dominant roles.

Similar notions pertain regarding $2 \mathrm{G}$. Most notably, some components - such as 2(2)68 and 2(6)248 correlate with interactions between isomers.

We explore the case for which $\iota_{I}$ is 36 .

Concepts similar to concepts pertaining for the case for which $\iota_{I}$ is six pertain. However, for any one isomer of a span-one elementary particle (such as a $2 \mathrm{~J}$ boson), the span of six correlating with $4(6) \mathrm{G} 4$ is, in effect, orthogonal to the span of six correlating with $2(6) 248$.

\subsubsection{Baryon asymmetry}

We explore the notion that the universe transited from an early state that did not exhibit baryon asymmetry to a later state that exhibits baryon asymmetry. 
To the extent that the early universe featured essentially the same number of antimatter quarks as matter quarks, something happened to create baryon asymmetry. The two-word term baryon asymmetry correlates with the present lack, compared to matter quarks, of antimatter quarks.

Aspects of ongoing modeling consider that early in the universe baryon symmetry pertained. Unverified ongoing modeling posits mechanisms that might have led to asymmetry. Some conjectured mechanisms would suggest asymmetries between matter simple fermions and antimatter simple fermions. One set of such simple fermions might feature the neutrinos. (See reference [18].)

Proposed modeling suggests scenarios that might have led to baryon asymmetry.

In one scenario, the interactions that equations $(167)$ and $(168)$ show pertain. This scenario converts three antimatter fermions into one matter fermion. Equation (169) shows an overall result. (Regarding equation (167) and to the extent that one wants to try to impose notions of conservation of lepton number and conservation of baryon number, the notion of $2 \mathrm{~T}_{+2 ; 0,-2}^{-1}$ would pertain. Regarding equation (168) and to the extent that one wants to try to impose notions of conservation of lepton number and conservation of baryon number, the notion of $2 \mathrm{~T}_{+2 ;+3,+1}^{-1}$ would pertain.) Baryon asymmetry would arise because reactions such as equations (167) and (168) show dominated compared to similar reactions that involve antiparticles to the particles that equations (167) and (168) show. Domination might correlate with an occurrence of more $2 \mathrm{~T}_{+2}^{-1}$; lasing than $2 \mathrm{~T}_{-2}^{+1}$; lasing.

$$
\begin{gathered}
1 \mathrm{Q}_{+1 ; 0,-1}^{+1}+1 \mathrm{Q}_{+1 ; 0,-1}^{-2} \rightarrow 2 \mathrm{~T}_{+2 ;}^{-1} \\
1 \mathrm{C}_{-3 ;-3,0}^{+3}+2 \mathrm{~T}_{+2 ;}^{-1} \rightarrow 1 \mathrm{Q}_{-1 ; 0,+1}^{+2} \\
1 \mathrm{C}_{-3 ;-3,0}^{+3}+1 \mathrm{Q}_{+1 ; 0,-1}^{+1}+1 \mathrm{Q}_{+1 ; 0,-1}^{-2} \rightarrow 1 \mathrm{Q}_{-1 ; 0,+1}^{+2}
\end{gathered}
$$

A threshold energy might be in or above the range of $208 \mathrm{GeV}$ to $213 \mathrm{GeV}$. (See equation (99).) A corresponding temperature is about $2 \times 10^{15}$ degrees Kelvin. As far as we know, this result - regarding temperature or (equivalently) regarding time after the Big Bang - is not inconsistent with core ongoing modeling.

We note two possible causes for an occurrence of more $2 \mathrm{~T}_{+2}^{-1}$; lasing than $2 \mathrm{~T}_{-2}^{+1}$; lasing. Possibly, the imbalance occurred, in effect, statistically at the time that baryon asymmetry first appeared. Possibly, earlier, an imbalance regarding production of left circularly polarized 8G2468 and right circularly polarized 8 G2468 occurred - perhaps statistically. (Note equation (165).) To the extent that effects correlating with such residual 8 G2468 remained long enough, some differences in energy distributions between some types of matter fermions and the corresponding types of antimatter fermions might have occurred. Those differences might have driven the asymmetry in tweak lasing.

We explore a concept that involves isomers.

Table 98 suggests possibilities for taking a multiple-isomer view of baryon asymmetry. We consider PR6ISP modeling. In this view, the lepton range $9 \leq M^{\prime \prime} \leq 12$ and quark range $9 \leq M^{\prime \prime} \leq 11$ might provide for an antimatter-centric complement to the matter-centric lepton range $0 \leq M^{\prime \prime} \leq 3$ and quark range $0 \leq M^{\prime \prime} \leq 2$. Similar results pertain for each of the two pairs $n=1$-and- $n=4$ and $n=2$-and$n=5$. With this view, there may be no need to posit interactions that led to baryon asymmetry. A similar conclusion can pertain regarding PR36ISP modeling. This essay does not further explore details regarding or implications of this concept.

\subsubsection{Filaments and baryon acoustic oscillations}

Proposed modeling is compatible with the ongoing modeling notion that ordinary matter baryon acoustic oscillations contributed to the formation of filaments.

Regarding models for which $\iota_{I}$ (as in $\mathrm{PR} \iota_{I} \mathrm{ISP}$ ) exceeds one, each of the five dark matter isomers has its own baryon-like particles and its own 2(1)G physics. Proposed modeling suggests, for models for which $\iota_{I}$ exceeds one, that dark matter baryon-like acoustic oscillations occurred in the early universe. Proposed modeling suggests that dark matter baryon-like acoustic oscillations contributed (along with ordinary matter baryon acoustic oscillations) to the formation of filaments.

\subsubsection{Amounts of clumping for large clumps of ordinary matter gas and of dark matter}

Reference [33] discusses observations that point to the notion that clumping of matter - ordinary matter gas and dark matter - might be less than ongoing modeling models suggest. The article alludes to a dozen observational studies and points to three papers - reference [34], reference [35], and communication 124a. For example, the last one of the three references studies distortions regarding images of galaxies. 
The work studies amounts of clumping of - for example - dark matter along the path that the observed light took. Clumps would be - to use wording from reference [33] - too thin. (Reference [33] suggests a result of too thin by about ten percent. This essay does not explore the topic of quantifying such thinness.) A distribution of galaxies would be - to use wording from reference [36 - too smooth. The reference suggests a notion of ten percent more evenly spread than ongoing modeling predicts.

Proposed modeling suggests that such effects might correlate with the notion that 4(2)G48 repels more stuff than would 4(1)G48. (See table 102.) Early formation of clumps correlates with 4(1)G246 attraction. Early clumps correlate with single isomers. Effects of 4(2)G48 repulsion would dilute matter around early clumps more than would effects that ongoing modeling might correlate with, in effect, 4(1)G48 repulsion. Effects of dilution might carry into the times for which $4(6) \mathrm{G} 4$ attraction dominates and leads to the clumps to which observations pertain.

\subsubsection{Galaxy clusters - ratios of dark matter to ordinary matter}

Regarding some galaxy clusters, people report inferred ratios of dark matter amounts to ordinary matter amounts.

References [37] and [38] report ratios of five-plus to one. The observations have bases in gravitational lensing. Reference [39] reports, for so-called massive galaxy clusters, a ratio of roughly 5.7 to one. (Perhaps, note reference [40.) The observations have bases in X-ray emissions.

Proposed modeling is not incompatible with these galaxy cluster centric ratios. Either one of PR6ISP modeling and PR36ISP modeling can pertain.

Reference [41] suggests a formula that correlates - across 64 galaxy clusters - dark matter mass, hot gas baryonic mass (or, essentially, ordinary matter mass), and two radii from the centers of each galaxy cluster. The reference suggests that the formula supports the notion of a correlation between dark matter and baryons. This essay de-emphasizes discussing the extent to which proposed modeling comports with this formula. Proposed modeling might suggest a correlation, based on proposed similarities between most dark matter and ordinary matter.

\subsubsection{Galaxy clusters - an explanation for aspects of the Bullet Cluster}

We consider either PR6ISP modeling or PR36ISP modeling. For each case, there are five dark matter isomers and one ordinary matter isomer.

Possibly, the evolution of each one of the six isomers paralleled the evolution of each of the other five isomers.

Such parallel evolution might lead to difficulties regarding explaining observations regarding the socalled Bullet Cluster.

People use the two-word term Bullet Cluster to refer, specifically, to one of two galaxy clusters that collided and, generally, to the pair of galaxy clusters. The clusters are now moving away from each other. Ongoing modeling makes the following interpretations based on observations. For each of the two clusters, dark matter continues to move along trajectories generally consistent with just gravitational interactions. For each of the two clusters, stars move along trajectories generally consistent with just gravitational interactions. For each of the two clusters, (ordinary matter) gas somewhat generally moves along with the cluster, but generally lags behind the other two components (dark matter and stars). Regarding such gas, people use the acronym IGM and the two-word term intergalactic medium. Ongoing modeling suggests that the IGM component of each original cluster interacted electromagnetically with the IGM component of the other original cluster. Electromagnetic interactions led to slowing the motion of the gas.

If each of the six dark matter or ordinary matter isomers evolved similarly, there might be problems regarding explaining aspects of the Bullet Cluster. One might expect that, in each galaxy cluster, more (than the observed amount of) dark matter would lag. The lag would occur because of one-isomer 2Gmediated interactions within each of the five dark matter isomers. Possibly, for each dark matter isomer, there would not be enough star-related stuff to explain the amount of dark matter that is not lagging. Possibly, there would not be enough $1 \mathrm{R} \otimes 2 \mathrm{U}$ dark matter to significantly help regarding explaining the amount of dark matter that is not lagging.

We assume that four dark matter isomers correlate with proposed modeling notions of cool dark matter and that one dark matter isomer exhibits behavior similar to behavior that ordinary matter exhibits. (See discussion related to table 98 and see table 100.)

Proposed modeling suggests that, for each of the two galaxy clusters, essentially all the stuff correlating with isomers one, two, four, and five would pass through the collision with just gravitational interactions having significance. For isomer three, incoming $1 \mathrm{R} \otimes 2 \mathrm{U}$ would pass through. For isomer zero, incoming 
Table 104: Aspects regarding a collision between two galaxy clusters (with the assumption that each of the two galaxy clusters has not undergone earlier collisions; and with the assumption that case S pertains, as per table 100)

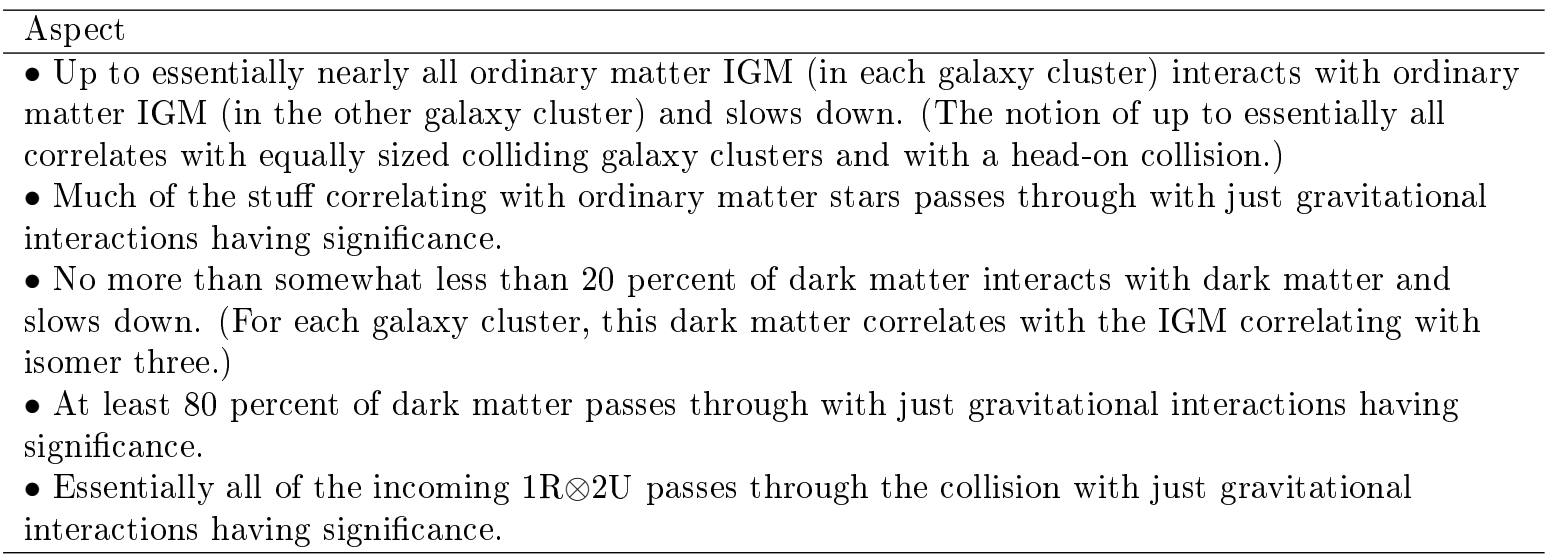

$1 \mathrm{R} \otimes 2 \mathrm{U}$ (which measures as dark matter) would pass through. Thus, at least 80 percent of the incoming dark matter would pass through the collision with just gravitational interactions having significance.

Table 104 lists aspects regarding a collision between two galaxy clusters. Here, we assume that each of the two galaxy clusters has not undergone earlier collisions. Here, we assume that case S pertains. (See table 100.)

We suggest that these proposed modeling notions might comport with various possible findings about IGM after a collision such as the Bullet Cluster collision. The findings might point to variations regarding the fractions of IGM that, in effect, stay with outgoing galaxy clusters and the fractions of IGM that, in effect, detach from outgoing galaxy clusters.

We discuss possible aspects regarding an outgoing galaxy cluster.

Suppose that, before a collision, ordinary matter IGM comprised much of the ordinary matter in the galaxy cluster. Suppose that, because of the collision, the galaxy cluster has a significant net loss of ordinary matter IGM. After the collision, the galaxy cluster could have a (perhaps somewhat arbitrarily) large ratio of amount of dark matter to amount of ordinary matter.

To the extent that IGM detaches from galaxy clusters after the galaxy clusters collide, the detached IGM might form one or more objects. Some such objects might have roughly equal amounts of dark matter and ordinary matter. The dark matter would correlate with isomer three.

\subsubsection{Galaxies - formation}

We discuss galaxy formation scenarios.

We assume that nature comports with at least one of PR6ISP modeling and PR36ISP modeling. (Neither ongoing modeling nor PR1ISP modeling includes the notion of more than one dark matter isomer. Regarding each of ongoing modeling and PR1ISP modeling, we think that it would be, at best, difficult to explain - based on for example $1 \mathrm{R} \otimes 2 \mathrm{U}$ dark matter - ratios, that observations suggest, of dark matter amounts to ordinary matter amounts.) For now, we de-emphasize some phenomena such as collisions between galaxies.

We anticipate that such galaxy formation and evolution scenarios will explain galaxy centric data that table 95 shows.

Models for galaxy formation and evolution might take into account the following factors - one-isomer repulsion (which correlates with the $4 \mathrm{G} 2468 \mathrm{a}$ and $4 \mathrm{G} 2468 \mathrm{~b}$ solutions), one-isomer attraction (which correlates with 4G246), two-isomer repulsion (which correlates with 4G48), six-isomer attraction (which correlates with 4G4), dissimilarities between isomers, the compositions of filaments and galaxy clusters, statistical variations in densities of stuff, and collisions between galaxies. Modeling might feature a notion of a multicomponent fluid with varying concentrations of gas-like or dust-like components and of objects (such as stars, black holes, galaxies, and galaxy clusters) for which formation correlates significantly with six-isomer (or 4G4) attraction.

We focus on early-stage galaxy formation and evolution. For purposes of this discussion, we assume that we can de-emphasize collisions between galaxies. We suggest the two-word term untouched galaxy for a galaxy that does not collide, before and during the time relevant to observations, with other galaxies. We emphasize formation scenarios and evolution scenarios for untouched galaxies. (Communication 124b and communication $124 \mathrm{c}$ discuss data that pertains regarding a time range from about one billion years 
Table 105: A scenario for the formation and evolution of a galaxy for which the original clump contains essentially just one isomer

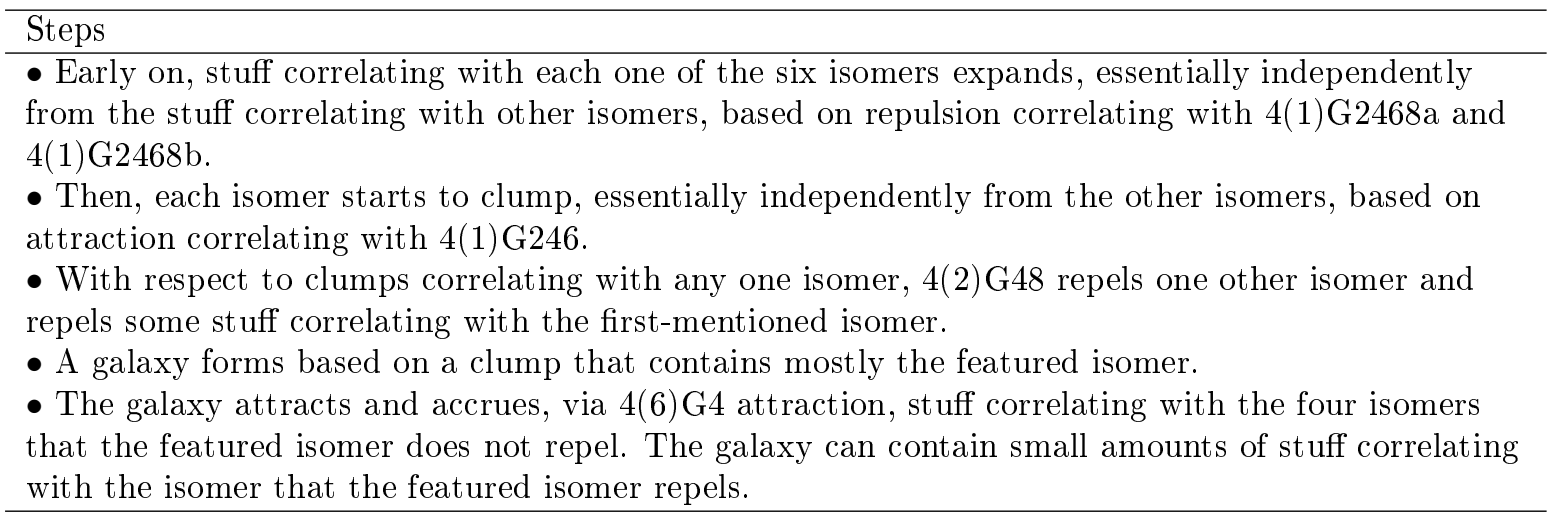

after the Big Bang to about 1.5 billion years after the Big Bang. Observations suggest that, out of a sample of more than 100 galaxies or galaxy-like rotating disks of material, about 15 percent of the objects might have been untouched.)

We assume that differences - in early evolution - regarding the various isomers do not lead, for the present discussion, to adequately significant differences - regarding $4 \mathrm{G}$ interactions and galaxy formation - between isomers. (We think that this assumption can be adequately useful, even given results that table 100 shows and our discussion regarding the Bullet Cluster. Regarding the Bullet Cluster, see discussion related to table 104.)

We organize this discussion based on the isomer or isomers that originally clump based, respectively, on $4 \mathrm{G} 246$ attraction or on 4G246 attraction and 4G4 attraction. Each one of some galaxies correlates with an original clump that correlates with just one isomer. Multi-isomer original clumps are possible. Because of $4 \mathrm{G} 48$ repulsion, an upper limit on the number of isomers that an original clump features might be three.

Table 105 discusses a scenario for the formation and evolution of a galaxy for which the original clump contains essentially just one isomer. Regarding this isomer, we use the word featured. We assume that PR6ISP modeling pertains. We assume that stuff that will become the galaxy is always in somewhat proximity with itself. We assume that no collisions between would-be galaxies or between galaxies occur.

\subsubsection{Galaxies - ratios of dark matter stuff to ordinary matter stuff}

We continue to explore the realm of one-isomer clumps.

One of two cases pertains. For so-called case A, one isomer of $4 \mathrm{G} 48$ spans (or connects) isomers zero and three. (Regarding numbering for isomers, see $n$ in table 100.) For so-called case B, one isomer of $4 \mathrm{G} 48$ spans isomer zero and one isomer out of isomers one, two, four, and five. The existence of many spiral galaxies might point to the notion that case A pertains. (Compare the rightmost column in table 106a and the rightmost column in table 106b.) However, we consider the possibility that people might not know of data or current modeling that would adequately point to the one of case A and case B that pertains. We discuss both cases.

Table 106 pertains. (See table 95.) The following sentences illustrate the notion that some statements in table 106 are at least somewhat conceptual. We assume that local densities for the isomers are somewhat the same. We assume that the galaxy remains adequately untouched. For each row in the table, OM stars can form (and become visible) over time, whether or not significant OM star formation occurs early on. The notation DMA:OMA $=1: 0^{+}$denotes the notion that the ratio of OMA to DMA might be arbitrarily small. The notion of three or four DM isomers in a halo refers to the notion that one or zero (respectively) of the DM isomers in the halo is the featured isomer. We de-emphasize some aspects regarding $1 \mathrm{R} \otimes 2 \mathrm{U}$ hadron-like particles.

Table 106 reflects at least two assumptions. Each core clump features one isomer. Each galaxy does not collide with other galaxies. Yet, data of which we know and discussion below seem to indicate that ratios that table 106 features pertain somewhat broadly. We think that galaxies that have core clumps that feature more than one isomer are more likely to appear as elliptical galaxies (and not as spiral galaxies) than are galaxies that have core clumps that feature only one isomer. Such likelihood can correlate with starting as being elliptical. Such likelihood can correlate with earlier transitions from spiral to elliptical. 
Table 106: Aspects regarding untouched galaxies that correlate with original one-isomer clumps (with just one of cases A and $\mathrm{B}$ pertaining to all galaxies)

(a) Case A

\begin{tabular}{|c|c|c|c|}
\hline Label & $\begin{array}{l}\text { Featured } \\
\text { isomer }(n)\end{array}$ & Early aspects regarding the galaxy & $\begin{array}{l}\text { Possible later aspects regarding } \\
\text { the galaxy }\end{array}$ \\
\hline $\mathrm{A} 0$ & 0 & $\begin{array}{l}\text { Forms some ordinary matter stars } \\
\text { early on. Starts at } \\
\text { DMA:OMA }=0^{+}: 1\end{array}$ & $\begin{array}{l}\text { Attracts cool dark matter over } \\
\text { time. Can get to DMA:OMA } \approx 4: 1 \text {, } \\
\text { with most DM in a halo. Might be } \\
\text { a spiral galaxy. }\end{array}$ \\
\hline A3 & 3 & $\begin{array}{l}\text { Forms some dark matter stars } \\
\text { early on. Starts at } \\
\text { DMA:OMA }=1: 0^{+}\end{array}$ & $\begin{array}{l}\text { Attracts the four other DM } \\
\text { isomers over time. Some OM stars } \\
\text { can form over time. Can settle at } \\
\text { DMA:OMA }=1: 0^{+} \text {. The } \\
\text { three-word term dark matter } \\
\text { galaxy pertains. }\end{array}$ \\
\hline $\mathrm{AX}$ & $1,2,4$, or 5 & $\begin{array}{l}\text { Might form dark matter stars } \\
\text { early on. Starts at } \\
\text { DMA:OMA }=1: 0^{+}\end{array}$ & $\begin{array}{l}\text { Attracts the OM isomer and three } \\
\text { other isomers over time. OM stars } \\
\text { can form over time. Can get to } \\
\text { DMA:OMA } \approx 4: 1 \text {, with three or } \\
\text { four DM isomers in a halo. Might } \\
\text { become an elliptical galaxy. }\end{array}$ \\
\hline
\end{tabular}

(b) Case B

\begin{tabular}{|c|c|c|c|}
\hline Label & $\begin{array}{l}\text { Featured } \\
\text { isomer }(n)\end{array}$ & Early aspects regarding the galaxy & $\begin{array}{l}\text { Possible later aspects regarding } \\
\text { the galaxy }\end{array}$ \\
\hline B0 & 0 & $\begin{array}{l}\text { Forms some ordinary matter stars } \\
\text { early on. Starts at } \\
\text { DMA:OMA }=0^{+}: 1\end{array}$ & $\begin{array}{l}\text { Attracts isomer three and three } \\
\text { cool dark matter isomers over } \\
\text { time. Can get to DMA:OMA } \approx 4: 1 \text {, } \\
\text { with three DM isomers in a halo. } \\
\text { Might appear to be an elliptical } \\
\text { galaxy. }\end{array}$ \\
\hline $\mathrm{BP}$ & $\begin{array}{l}\text { The DM } \\
\text { isomer that } \\
4 \mathrm{G} 48 \text { connects } \\
\text { to the OM } \\
\text { isomer }\end{array}$ & $\begin{array}{l}\text { Might form dark matter stars } \\
\text { early on. Starts at } \\
\text { DMA:OMA }=1: 0^{+} .\end{array}$ & $\begin{array}{l}\text { Attracts the other DM isomers } \\
\text { over time. OM stars can form over } \\
\text { time. Can settle at } \\
\text { DMA:OMA }=1: 0^{+} \text {. The } \\
\text { three-word term dark matter } \\
\text { galaxy pertains. }\end{array}$ \\
\hline B3 & 3 & $\begin{array}{l}\text { Forms some dark matter stars } \\
\text { early on. Starts at } \\
\text { DMA:OMA }=1: 0^{+}\end{array}$ & $\begin{array}{l}\text { Attracts the OM isomer and three } \\
\text { other DM isomers over time. OM } \\
\text { stars can form over time. Can get } \\
\text { to DMA:OMA } \approx 4: 1 \text {, with three } \\
\text { DM isomers in a halo. Might } \\
\text { appear to be an elliptical galaxy. }\end{array}$ \\
\hline BY & $\begin{array}{l}\text { Any one of the } \\
\text { other three } \\
\text { DM isomers }\end{array}$ & $\begin{array}{l}\text { Might form dark matter stars } \\
\text { early on. Starts at } \\
\text { DMA:OMA }=1: 0^{+}\end{array}$ & $\begin{array}{l}\text { Attracts the OM isomer and three } \\
\text { other DM isomers over time. OM } \\
\text { stars can form over time. Can get } \\
\text { to DMA:OMA } \approx 4: 1 \text {, with three or } \\
\text { four DM isomers in a halo. Might } \\
\text { appear to be an elliptical galaxy. }\end{array}$ \\
\hline
\end{tabular}


We explore the extent to which the galaxy formation scenario comports with observations.

Observations regarding stars and galaxies tend to have bases in ordinary matter isomer $2 \mathrm{G}$ phenomena (or, readily observable electromagnetism). (The previous sentence de-emphasizes some observations regarding collisions between black holes or neutron stars - that have bases in 4G phenomena.) People report ratios of amounts of dark matter to amounts of ordinary matter.

We discuss observations correlating with early in the era of galaxy formation. Table 95 comports with these results. We suggest that visible early galaxies correlate with generalization of label-A0 or with generalization of label-B0. (See table 106.) Label-A3 or label-B3 evolves similarly to label-A0 or label-B0, but is not adequately visible early on.

- Reference [42] provides data about early stage galaxies. (See, for example, figure 7 in reference [42]. The figure provides two graphs. Key concepts include redshift, stellar mass, peak halo mass, and a stellar - peak halo mass ratio.) Data correlating with redshifts of at least seven suggests that some galaxies accrue, over time, dark matter, with the original fractions of dark matter being small. Use of reference 43 suggests that redshifts of at least seven pertain to times ending about 770 million years after the Big Bang.

- Reference 44 reports zero-plus to one ratios. The observations have bases in the velocities of stars within galaxies and correlate with the three-word term galaxy rotation curves. Proposed modeling suggests that the above galaxy evolution scenario comports with this data.

We discuss observations correlating with later times. Table 95 comports with these results.

- Reference 45 discusses some MED09 spiral - or, disk - galaxies. A redshift of approximately $z=1.57$ pertains. (See reference [46.) The redshift correlates with a time of 4.12 billion years after the Big Bang. (We used reference [43] to calculate the time.) Reference [45] reports ratios of amount of dark matter to amount of ordinary matter of approximately four to one. The observations have bases in gravitational lensing. We suggest that each label - other than label-A3 or label-BP that table 106 shows might pertain. (We note, without further comment, that this example might correlate with the notion that case A pertains to nature and that case B does not pertain to nature. This example features spiral galaxies. Label-A0 suggests a correlation with spiral galaxies. Each other label - pertaining to case A or to case B - either correlates with dark matter galaxies or might suggest a correlation with - at least statistically - evolution into elliptical galaxies. See table 106.)

- To the extent that such an MED09 galaxy models as being nearly untouched, proposed modeling offers the following possibility. The galaxy began based on a one isomer clump. The clump might have featured the ordinary matter isomer. The clump might have featured a dark matter isomer that does not repel ordinary matter. Over time, the galaxy accrued stuff correlating with the isomers that the original clump did not repel. Accrual led to a ratio of approximately four to one.

- To the extent that such an MED09 galaxy models as not being untouched, proposed modeling offers the following possibility. One type of collision merges colliding galaxies. One type of collision features galaxies that separate after exchanging material. For either type of collision, incoming galaxies having approximately four times as much dark matter as ordinary matter might produce outgoing galaxies having approximately four times as much dark matter as ordinary matter.

- Reference 47 discusses the Dragonfly 44 galaxy. A redshift of $z=0.023$ pertains. The redshift correlates with a time of 13.45 billion years after the Big Bang. (We used reference [43] to calculate the time.) People discuss the notion that ordinary matter accounts for perhaps as little as one part in 10 thousand of the matter in the galaxy. (See reference 48.) The observations have bases in light emitted by visible stars. This case correlates with the three-word term dark matter galaxy. We suggest that label-A3 or label-BP might pertain. (See table 106.)

The following notions pertain regarding other data of which we know. Here, the ratios are ratios of dark matter amounts to ordinary matter amounts. Table 95 seems to comport with these results. (See table 106.)

- Reference 49 discusses six baryon-dominated ultra-diffuse galaxies that seem to lack dark matter, at least to the radius studied by gas kinematics via observations of light with a wavelength of 21 centimeters. These observations seem not to be incompatible with the early stages of label-A0 or label-B0. 
- Reference [50 discusses 19 dwarf galaxies that lack having much dark matter, from their centers to beyond radii for which ongoing modeling suggests that dark matter should dominate. These observations measure r-band light that the galaxies emitted. These observations seem not to be incompatible with the early stages of label-A0 or label-B0.

- People report two disparate results regarding the galaxy NGC1052-DF2. Proposed modeling seems to be able to explain either ratio. Proposed modeling might not necessarily explain ratios that would lie between the two reported ratios.

- Reference [51] suggests a ratio of much less than one to one. The observation has bases in the velocities of stars - or, galaxy rotation curves. This observation seems not to be incompatible with the early stages of label-A0 or label-B0.

- Reference [52 suggests that at least 75 percent of the stuff within the half mass radius is dark matter. This ratio seems similar to ratios that reference [45] discusses regarding some MED09 galaxies. (See discussion above regarding MED09 galaxies.) We suggest that each label - other than label-A3 or label-BP - that table 106 shows can pertain.

- The galaxy NGC1052-DF4 might correlate with a ratio of much less than one to one. (See reference [53.) The observation has bases in the velocities of stars - or, galaxy rotation curves. This observation seems not to be incompatible with the early stages of label-A0 or label-B0.

- The compact elliptical galaxy Markarian 1216 has an unexpectedly large amount of dark matter in its core and may have stopped accumulating each of ordinary matter and dark matter approximately 4 billion years after the Big Bang. (See reference [54.) Observations feature the X-ray brightness and temperature of hot gas. This galaxy might correlate with an original clump that features three isomers. One isomer would be the ordinary matter isomer. Around the time that the galaxy stopped accruing material, there was - near the galaxy - essentially nothing left for the galaxy to attract via $4(6) \mathrm{G} 4$.

- The galaxy XMM-2599 stopped producing visible stars by approximately 1.8 billion years after the Big Bang. (See reference [55].) People speculate regarding a so-called quenching mechanism. Proposed modeling suggests that phenomena similar to phenomena that might pertain regarding Markarian 1216 might pertain regarding XMM-2599.

People report other data. Table 95 and table 106 seem not to be incompatible with these results. We are uncertain as to the extents to which proposed modeling provides insight that ongoing modeling does not provide.

- One example features a rotating disk galaxy, for which observations pertain to the state of the galaxy about 1.5 billion years after the Big Bang. (See reference [56.) People deduce that the galaxy originally featured dark matter and that the galaxy attracted ordinary matter.

- One example features so-called massive early-type strong gravitation lens galaxies. (See reference [57.) Results suggest, for matter within one so-called effective radius, a minimum ratio of dark matter to dark matter plus ordinary matter of about 0.38. Assuming, for example, that measurements correlating with material within larger radii would yield larger ratios, these observational results might support the notion that the galaxies accumulated dark matter over time.

- One example pertains to early stages of galaxies that are not visible at visible light wavelengths. (See reference [58].) Observations feature sub-millimeter wavelength light. We might assume that proposed modeling galaxy formation scenarios comport with such galaxies. We are not certain about the extent to which proposed modeling might provide insight regarding subtleties, such as regarding star formation rates, correlating with this example.

- We are uncertain as to the extent to which proposed modeling might provide insight regarding possible inconsistencies - regarding numbers of observed early stage galaxies and numbers of later stage galaxies - that correlate with various observations and models. (For a discussion of some possible inconsistencies, see reference [59].)

- We are uncertain as to the extent to which proposed modeling might provide insight regarding the existence of two types - born and tidal - of ultra-diffuse galaxies. (See reference [60].)

Observations that we discuss above indicate that some galaxies do not exhibit dark matter halos. Proposed modeling that we discuss above comports with the notion that some galaxies do not exhibit dark matter halos. 


\subsubsection{Aspects regarding some components of galaxies}

We discuss effects, within galaxies, that might correlate with dark matter.

Reference [61] reports, based on a study of 11 galaxy clusters, more instances of more gravitational lensing - likely correlating with clumps of dark matter that correlate with individual galaxies - than ongoing modeling simulations predict. (Perhaps, see reference [62].) Reference [62] suggests that the number of instances - 13 - compares with an expected number of about one. We suggest the possibility that the clumps might be dark matter galaxies. (See, for example, table 106.) Perhaps some of the dark matter galaxies are dwarf dark matter galaxies. We suggest the possibility that galaxies with significant amounts of ordinary matter gravitationally captured (or at least attracted) such dark matter clumps.

People study globular cluster systems within ultra-diffuse galaxies. Regarding 85 globular cluster systems in ultra-diffuse galaxies in the Coma cluster of galaxies, reference 63. suggests that 65 percent of the ultra-diffuse galaxies are more massive than people might expect based on ongoing modeling relationships, for so-called normal galaxies, between stellar mass and halo mass. We are uncertain as to the extent to which proposed modeling might explain this result. For example, proposed modeling might suggest that phenomena related to isomers might play a role. (See, for example, table 106.) Higher-mass galaxies might tend to feature more dark matter isomers (or tend to feature more material that correlates with such isomers) than do lower-mass galaxies.

Discussion related to table 106 is not incompatible with the notion that visible stars do not include much dark matter.

Discussion related to table 106 is not incompatible with the notion that some black holes that form based on the collapse of stars might originally correlate with single isomers. Discussion above is not incompatible with the notion that supermassive black holes might contain material correlating with more than one isomer. (Perhaps, note references [64] and 65].)

We suggest that proposed modeling might provide insight about other aspects regarding black holes. People suggest gaps in understanding about the formation of intermediate-mass and large-mass black holes. (Perhaps, note reference [66].) Proposed modeling suggests the possibility that the 4G(1)246 attractive component of G-family forces plays key roles in the early formation of some intermediate-mass and large-mass black holes.

Regarding the coalescing of two black holes, proposed modeling suggests that people might be able to estimate the extent to which $4 \mathrm{G} 48$ repulsion pertains. Effects of $4 \mathrm{G} 48$ repulsion would vary based on the amounts of various isomers that each black hole in a pair of colliding black holes features.

\subsubsection{Dark matter effects within the Milky Way galaxy}

People look for possible local effects, within the Milky Way galaxy, that might correlate with dark matter.

For one example, data regarding the stellar stream GD-1 suggests effects of an object of $10^{6}$ to $10^{8}$ solar masses. (See reference [67].) Researchers tried to identify and did not identify an ordinary matter object that might have caused the effects. The object might be a clump of dark matter. (See reference [68].) Proposed modeling offers the possibility that the object is an originally dark matter centric clump of stuff.

For other examples, people report inhomogeneities regarding Milky Way dark matter. (See references [68] and [69.) Researchers note that simulations suggest that such dark matter may have velocities similar to velocities of nearby ordinary matter stars. We suggest that these notions are not incompatible with proposed modeling notions of the existence of dark matter stars that would be similar to ordinary matter stars.

\subsubsection{High-mass neutron stars}

We discuss proposed modeling that might explain some aspects regarding high-mass neutron stars.

The following results have bases in observations. An approximate minimal mass for a neutron star might be $1.1 M_{\odot}$. (See reference [70.) The symbol $M_{\odot}$ denotes the mass of the sun. An approximate maximum mass for a neutron star might be $2.2 M_{\odot}$. (See references [71] and [72].) [72].)

Some ongoing modeling models suggest a maximum neutron star mass of about $1.5 M_{\odot}$. (See reference

Observations correlate with most known neutron star pairs having masses in the range that equation (170) shows and one neutron star pair having a mass of about 3.4 solar masses. (See references [73] and [74].) Here, $M$ denotes the mass of a pair. The 3.4 number results from the second detection via gravitational waves of a merger of two neutron stars. People assign the name GW190425 to that detection. 


$$
2.5 M_{\odot} \lesssim M \lesssim 2.9 M_{\odot}
$$

People speculate - based on, at least, the GW190425 result - about needs for new modeling regarding neutron stars. (See references [73] and [72].)

Detection GW190814 suggests that people have inferred the existence of an object for which equation (171) pertains. (See reference [75].) People speculate that the object might have been a high-mass neutron star or might have been a low-mass black hole.

$$
M \approx 2.6 M_{\odot}
$$

We discuss possible bases for high-mass neutron stars.

The PR6ISP span of $4 \mathrm{G} 4$ is six.

Some high-mass neutron stars might, in effect, result from mergers of neutron stars, with each merging neutron star correlating with an isomer that differs from the isomer pertaining to each other neutron star that forms part of the merger.

\subsubsection{Dark energy density}

We explore possible explanations for non-zero dark energy density.

Equation (172) shows an inferred ratio of present density of the universe of dark energy to present density of the universe of dark matter plus ordinary matter plus (ordinary matter) photons. (Reference [13] provides the four items of data.) We know of no inferences that would not comport with a somewhat steady increase, regarding the inferred ratio correlating with equation (172), from approximately zero, with time since somewhat after the Big Bang. (Communication 124d implies a ratio of approximately zero correlating with 380 thousand years after the Big Bang. Also, inferences that reference [76] discusses might suggest that dark energy density increases with time.)

$$
\Omega_{\Lambda} /\left(\Omega_{\mathrm{c}}+\Omega_{\mathrm{b}}+\Omega_{\gamma}\right) \approx 2.3
$$

Some aspects of ongoing modeling correlate inferred dark energy densities of the universe with phenomena correlating with terms such as vacuum energy, vacuum fluctuations, or quintessence. Proposed modeling is not necessarily incompatible with notions such as vacuum energy. However, double-entry aspects of proposed modeling point to possibilities for modeling that does not embrace notions such as vacuum energy.

Aspects related to aye (or, 0I) bosons and jay (or, 2J) bosons might lead to phenomena similar to effects that ongoing modeling correlates with vacuum energy, vacuum fluctuations, or quintessence. (See discussion related to equations (123) and (124). Note that, for much of the history of the universe, large-scale effects of $0 \mathrm{I}$ and $2 \mathrm{~J}$ bosons might be small compared to other phenomena.) Ongoing modeling correlates some of those - that is, vacuum energy, vacuum fluctuations, or quintessence - effects with data about dark energy densities. To the extent that phenomena correlating with aye and jay bosons suffice to explain dark energy densities, there might not be a need to consider PR36ISP modeling. Assuming that such phenomena might not adequately explain non-zero dark energy density, we discuss possibilities for other proposed modeling aspects that might explain non-zero dark energy density.

For PR6ISP modeling, proposed modeling includes the notion of 2(6)G248, whereas ongoing modeling correlates with the notion of 2(1)G248. We suggest that the difference, in proposed modeling, between 2(6)G248 and 2(1)G248 might correlate with nature's indirectly producing effects, regarding CMB, that people correlate, via ongoing modeling, with some non-zero dark energy density. The difference correlates with interactions between ordinary matter and dark matter.

For PR36ISP modeling, differences between 2(>1)G $\mathrm{G}$ and 2(1)G $\Gamma$ correlate with interactions between ordinary matter plus dark matter and doubly dark matter. Dark energy density might correlate with a notion of dark energy stuff. Dark energy stuff would correlate with 30 doubly dark matter isomers. Modeling suggests an upper bound of approximately five regarding, in effect, a possible future value for the ratio that correlates with equation (172).

\section{Discussion: core ongoing modeling and core proposed modeling}

This unit discusses possibilities for adding aspects of core proposed modeling to core ongoing modeling. 


\subsection{The elementary particle Standard Model}

We explore synergies between proposed modeling and the elementary particle Standard Model.

Equation (173) shows internal symmetries that correlate with the ongoing modeling elementary particle Standard Model. Proposed modeling might correlate with these symmetries. (Regarding $S U(3)$, see discussion regarding table 54. Regarding $S U(2)$, see discussion regarding table 42 . Regarding $U(1)$, see discussion regarding table 36 and discussion regarding table 42.)

$$
S U(3) \times S U(2) \times U(1)
$$

People might try to add to the Standard Model some of the symmetries that proposed modeling suggests. Examples include conservation of charge and rebuttable somewhat conservation of fermion generation.

People might try to add to the Standard Model some of the simple particles and root forces that proposed modeling suggests. Examples include the jay bosons and $8 \mathrm{G}$.

People might try to add to the Standard Model the $1 \mathrm{R} \otimes 2 \mathrm{U}$ hadron-like particles that proposed modeling suggests.

Proposed modeling might provide a basis for extending the Standard Model to include concepts related to mass and concepts related to forces that correlate with bosons that have spins of at least two.

This essay does not speculate regarding the extent to which people might find synergies between Lagrangian aspects of the Standard Model and modeling that proposed modeling suggests regarding refraction. (See discussion related to equation (79).)

This essay does not speculate regarding the extent to which people might be able to add concepts related to isomers to Lagrangian aspects of the Standard Model.

\subsection{Concordance cosmology}

We discuss aspects that people might want to add to concordance cosmology.

We note aspects that discussion elsewhere in this essay de-emphasizes.

- Early in the evolution of the universe, quarks, arcs, and gluons might have formed hadron-like seas. The seas might have undergone phase changes, with the last changes featuring at least one transition from seas to hadron-like particles.

- Scenarios regarding clumping suggest that a significant fraction of early black holes contained stuff correlating with essentially just one isomer. Regarding PR6ISP modeling, approximately one-sixth of such one-isomer black holes features ordinary matter and approximately five-sixths of such oneisomer black holes features dark matter.

- Proposed modeling is not necessarily incompatible with an ongoing modeling notion of possible large-scale flatness for the universe.

Table 107 suggests some phenomena that people might want to add to the cosmology timeline or for which people might want to add details to the cosmology timeline.

\subsection{Large-scale physics}

Ongoing modeling concepts that people use to try to model observed changes in the rate of expansion of the universe include the Hubble parameter (or, Hubble constant), equations of state (or, relationships between - at least - density and pressure), and general relativity.

While general relativity comports with various phenomena, people discuss possible problems regarding the applicability of general relativity to large-scale physics. (See, for example, reference [77.)

People suggest possible incompatibilities between observations and ongoing modeling models. (See, for example, reference [78], reference [79], reference [80, reference [36], and communication 124e. However, some people note possible objections to some notions of incompatibility. See, for example, references [81] and [82.) People suggest phenomenological remedies regarding the modeling. (See, for example, reference [83].)

Proposed modeling offers possible insight and resolution regarding such concerns.

We consider modeling that might pertain to large-scale phenomena for other than the very early universe. We assume that general relativity pertains regarding PR1ISP modeling, including $4 \mathrm{G}$ aspects of PR1ISP modeling.

We consider the case of PR6ISP modeling. 
Table 107: Some phenomena that people might want to add to the cosmology timeline or for which people might want to add details to the cosmology timeline

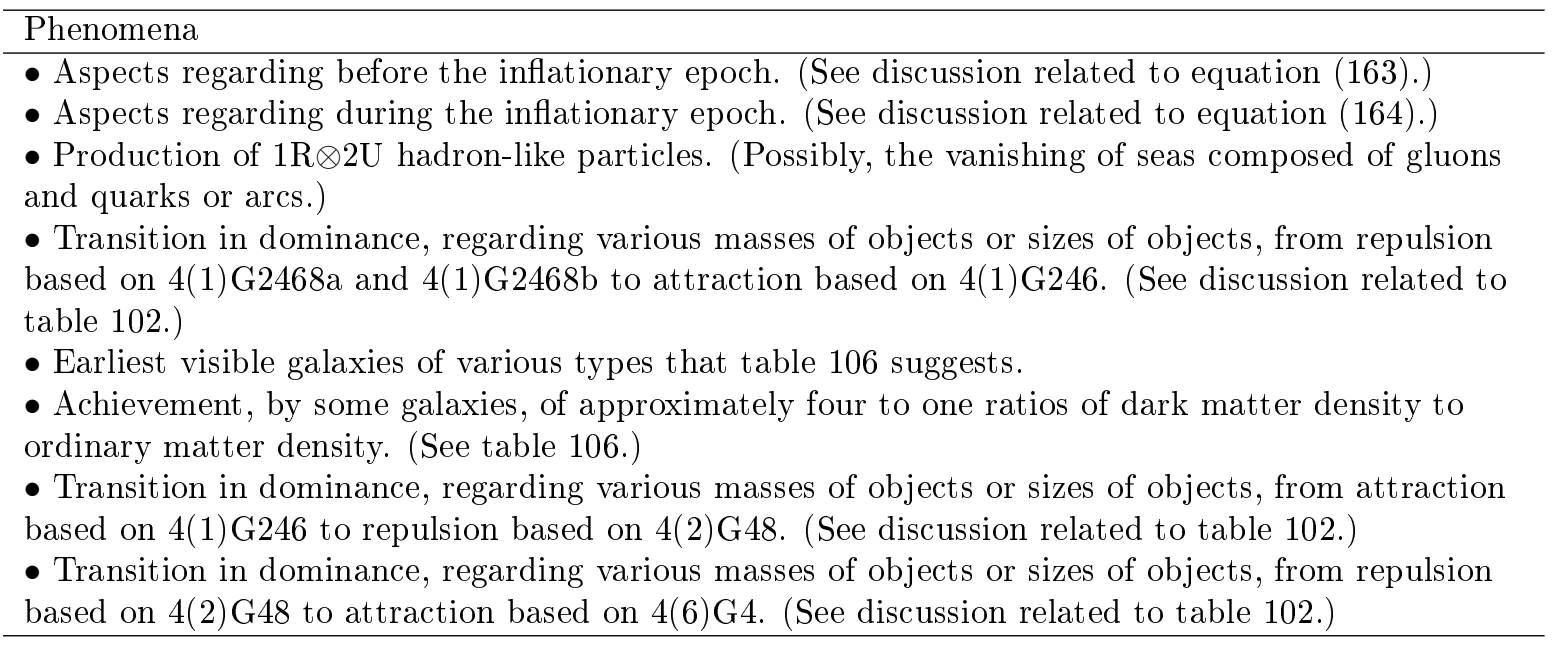

We assume that galaxy clusters tend to have equal amounts of stuff correlating with each of the six isomers.

We consider modeling that includes both the multi-billion-year era of decreasing rate of expansion of the universe and the current multi-billion-year era of increasing rate of expansion of the universe. The $4 \mathrm{G} 246$ attractive component of $4 \mathrm{G}$ has a span of one isomer. The $4 \mathrm{G} 48$ repulsive component of $4 \mathrm{G}$ has a span of two isomers. Tuning a model to the era of decreasing rate might produce a model that underestimates repulsive effects that lead to the increasing rate that correlates with the current era.

We generalize. Regarding the large-scale universe and motions of objects, one might need to limit applications of equations of state and general relativity to motions of objects that modeling can treat as having a span of six and as having roughly equal amounts of stuff correlating with each isomer.

We explore a possible concern regarding smaller objects.

We consider modeling regarding black holes and neutron stars. To the extent that a black hole or neutron star includes significant amounts of material correlating with each of at least two isomers, modeling - based on general relativity - for gravitational effects regarding high-outflow phenomena might be less than adequately accurate. Inaccuracy might occur, for example, to the extent that the outflow material does not interact via $4 \mathrm{G} 48$ with an isomer for which the black hole or neutron star has a significant amount. People observe high-outflow phenomena related to - for example - quasars, blazars, and pulsars.

We consider the case of PR36ISP modeling.

Six isomers of 4(6)G4 pertain. General relativity might pertain somewhat for each of the six PR6ISPlike isomers. The concept of geodesic motion would not pertain across PR6ISP-like isomers.

\subsection{Quantum electrodynamics regarding positronium}

Reference [11] reports a discrepancy between the observed energy correlating with one type of finestructure transition in positronium and a prediction based on core ongoing modeling. (See discussion regarding equation (84).)

Proposed modeling suggests the discrepancy correlates with a limit to the applicability of current core ongoing modeling. Table 70 suggests aspects that might correlate with that limitation. Features of the suggestion include the following notions. Core proposed modeling offers the opportunity to correlate the core ongoing modeling notion of a Pauli exclusion force with effects of jay bosons. Effects of jay bosons correlate with an interaction - between an electron and a positron bound together in positronium - for which ongoing modeling does not have an analog.

\subsection{The masses of $1 R \otimes 2 U$ hadron-like dark matter particles}

We discuss rest energies for $1 \mathrm{R} \otimes 2 \mathrm{U}$ hadron-like particles.

The rest energy of a proton does not differ much from the rest energy of a neutron. For hadrons composed of generation-one quarks, the masses of hadrons do not vary much based on the masses of the quarks or on the charges of the quarks. The rest energies of $1 \mathrm{R} \otimes 2 \mathrm{U}$ hadron-like particles that contain exactly three arcs might approximate the rest energy of the proton, which is about $938 \mathrm{MeV}$. (Reference 
Table 108: Possible rest energies for $1 \mathrm{R} \otimes 2 \mathrm{U}$ hadron-like particles

\begin{tabular}{l}
\hline Possible rest energies (in $\mathrm{GeV}$ ) for $1 \mathrm{R} \otimes 2 \mathrm{U}$ \\
\hline$\sim 0.06$ \\
$\sim 0.9$ \\
$\sim 4$ \\
Other.
\end{tabular}

Table 109: Instances in which core proposed modeling PFS models might seem to depend on - or at least might seem to reflect - aspects of core ongoing modeling KMS models

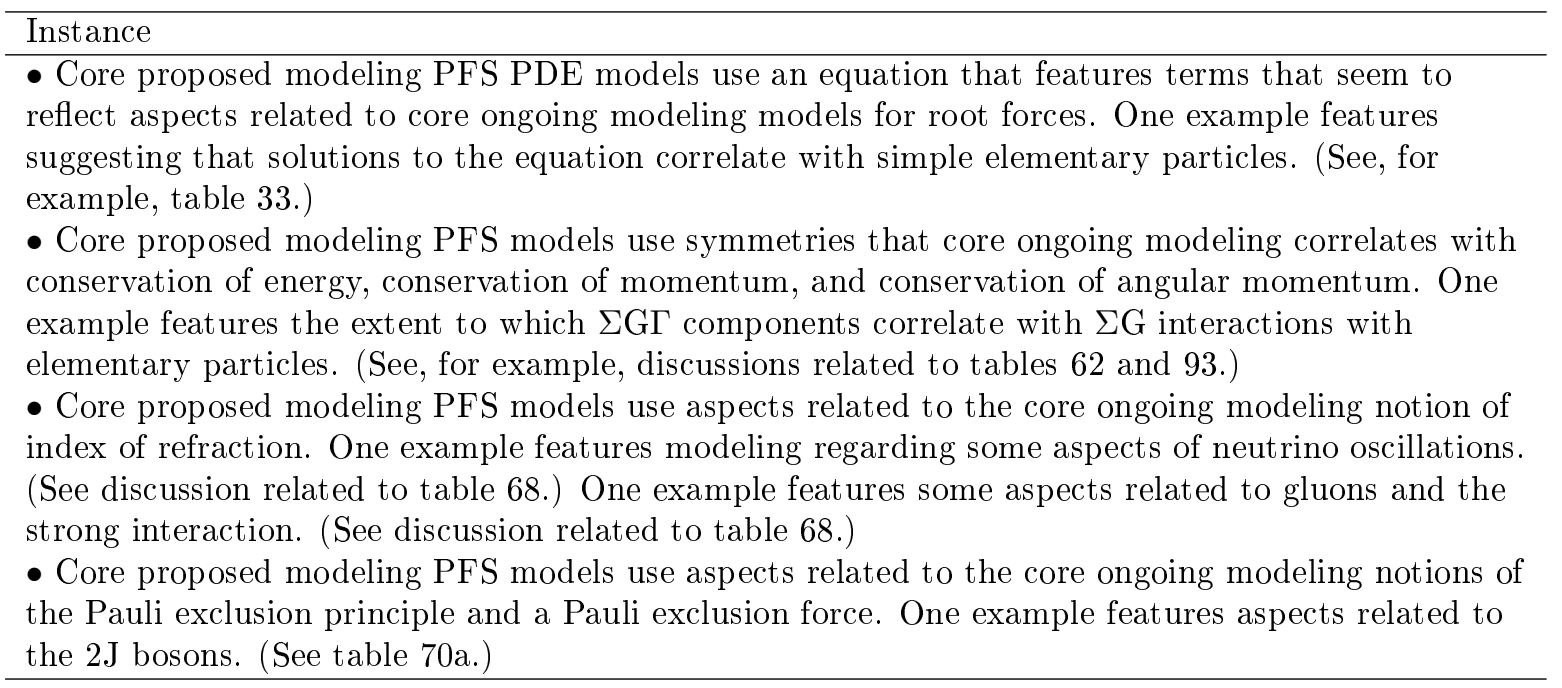

[13] provides data regarding hadron masses.) The rest energies of $1 \mathrm{R} \otimes 2 \mathrm{U}$ hadron-like particles that contain exactly two arcs might approximate the rest energy of the zero-charge pion, which is about 135 $\mathrm{MeV}$.

We explore another concept for estimating masses for $1 \mathrm{R} \otimes 2 \mathrm{U}$ hadron-like particles. The concept has bases in the relative densities of the universe of $1 \mathrm{Q} \otimes 2 \mathrm{U}$ hadrons and $1 \mathrm{R} \otimes 2 \mathrm{U}$ hadron-like particles.

Nature might have created concurrently, essentially, the current populations of $1 \mathrm{Q} \otimes 2 \mathrm{U}$ hadrons and $1 \mathrm{R} \otimes 2 \mathrm{U}$ hadron-like particles. We assume that each of $1 \mathrm{Q} \otimes 2 \mathrm{U}$ hadrons and $1 \mathrm{R} \otimes 2 \mathrm{U}$ hadron-like particles consists mainly of three-fermion particles.

Equation 174 might estimate the current relevant ratio of density of $1 \mathrm{R} \otimes 2 \mathrm{U}$ hadron-like particles to density of ordinary matter. (Regarding the symbol $\Omega_{1 \mathrm{R} 2 \mathrm{U}, 0}$ and the ratio $\Omega_{1 \mathrm{R} 2 \mathrm{U}, 0} / \Omega_{\mathrm{b}} \sim 0.055$, see discussion related to equation (154).) The symbol $m$ denotes the rest mass of a typical hadron-like particle. The leftmost use of the ratio $m_{1 \mathrm{R} \otimes 2 \mathrm{U}} / m_{1 \mathrm{Q} \otimes 2 \mathrm{U}}$ correlates with rest energy (or rest mass) per particle. The rightmost use of the ratio $m_{1 \mathrm{R} \otimes 2 \mathrm{U}} / m_{1 \mathrm{Q} \otimes 2 \mathrm{U}}$ occurs as the input to a calculation of an exponential and correlates with a hypothesis regarding the relative numbers of particles that nature created.

$$
0.055 \sim \Omega_{1 \mathrm{R} 2 \mathrm{U}, 0} / \Omega_{\mathrm{b}} \sim\left(m_{1 \mathrm{R} \otimes 2 \mathrm{U}} / m_{1 \mathrm{Q} \otimes 2 \mathrm{U}}\right) \exp \left(-m_{1 \mathrm{R} \otimes 2 \mathrm{U}} / m_{1 \mathrm{Q} \otimes 2 \mathrm{U}}\right)
$$

Two mathematical solutions exist. The respective solutions, expressed in terms of $m \_c^{2}$ and in units of $\mathrm{GeV}$ are $\sim 0.06$ and $\sim 4$.

Table 108 summarizes some possible rest energies for $1 \mathrm{R} \otimes 2 \mathrm{U}$ hadron-like particles.

\subsection{Relationships between core proposed modeling and core ongoing modeling}

We develop - elsewhere - some aspects of core proposed modeling in a manner that does not necessarily correlate with core ongoing modeling. However, the core proposed modeling list of suggested elementary particles might not necessarily be independent of core ongoing modeling, which tends to feature laws of motion.

Table 109 notes instances in which core proposed modeling PFS models might seem to depend on or at least might seem to reflect - aspects of core ongoing modeling KMS models. 


\section{Discussion: unverified ongoing modeling and core proposed modeling}

This unit discusses possibilities that core proposed modeling provides insight regarding unverified aspects of ongoing modeling.

\subsection{Supersymmetry}

Table 23 might suggest possibilities for some notion of symmetry based on equation 175 . Here, the symbol $\leftrightarrow$ correlates with notions of exchanging roles. For some relevant nonnegative values of $\Sigma$, table 23 might suggest possibilities for a relevant notion of symmetry based on equation (176).

$$
\begin{gathered}
m>0 \leftrightarrow m \doteq 0 \\
\Sigma \leftrightarrow \Sigma+1
\end{gathered}
$$

Tables 23 and 92 seem, in themselves, to be incompatible with supersymmetry. People might explore the notion of layering supersymmetry over results that tables 23 and 92 show. However, given aspects of proposed modeling, supersymmetry might not be necessary to explain known phenomena.

\subsection{String theory}

We discuss the notion that aspects of proposed modeling might help people explore the relevance of string theory to elementary particle physics.

We suggest perspective about string theory and about proposed modeling. (Reference [84] provides perspective about string theory.) Regarding simple particles and root forces, proposed modeling correlates with the three-word term modeling regarding what. Proposed modeling outputs a list of what elementary particles nature embraces or might embrace. We contrast notions of modeling regarding what with notions correlating with the three-word term modeling regarding how. Proposed modeling might not yet suggest modeling regarding how nature selects or forms elementary particles. Attempts to apply string theory to elementary particle physics might correlate with trying to develop modeling regarding how and trying to use the modeling regarding how to produce modeling regarding what.

We suggest that would-be applications of string theory to elementary particle physics, correlate with KMS modeling. Regarding physics modeling, proposed modeling adds, compared to ongoing modeling, the notion of PFS modeling. To the extent that string theory continues not to output a well-defined, potentially-complete list of elementary particles, people might want to explore reusing or extending string theory mathematics to incorporate PFS aspects along with KMS aspects.

People might want to explore possible correlations between string theory notions of so-called frothiness of space-time and aspects of PFS modeling.

PFS modeling includes notions of volume-like solutions that correlate with fields for elementary particles and notions of point-like solutions that correlate with particles for elementary particles. (See equations (60) and (61).) Here, in effect, transitions between volume-like and point-like can involve changes regarding a number, $D$, of dimensions. The numbers $D$ correlate with, at least, aspects of PFS modeling. Perhaps, people can interpret seeming transitions - regarding modeling - between volume-like and point-like as correlating with (at least, mathematical) notions of frothiness.

\subsection{Theory of everything}

We speculate that proposed modeling points toward possibilities for a superset of a so-called theory of everything.

People discuss the notion of modeling that would describe much of fundamental physics. Within this context, people use the three-word term theory of everything to allude to modeling that would unify quantum mechanics and general relativity. People use the one-element term ToE to abbreviate the three-word phrase theory of everything.

We suggest that such a use of the term ToE might correlate with overlooking key aspects of nature. In the context of proposed modeling and ongoing modeling, that use of the term ToE might correlate with a notion of a unified modeling of motion and might overlook the topic of objects that move.

Table 110 notes aspects of a possible superset of a so-called ToE.

The superset correlates, at the least, with the notion of modeling regarding what. The superset does not necessarily correlate with modeling regarding how nature, in effect, selects or creates properties of objects, selects or creates elementary particles, or selects or creates relevant aspects regarding motion. 
Table 110: Aspects of a possible superset of a so-called ToE (or, theory of everything)

Aspect

- Modeling that lists properties of elementary particles and of other objects.

- Modeling that lists elementary particles.

- Modeling that embraces or integrates modeling regarding motion.

- Modeling that unifies the aspects above.

People characterize some ongoing modeling candidates for a ToE by (mathematical) groups with which the candidates correlate.

We discuss the possibility that people can find a group theoretic statement that correlates with the superset. We structure this discussion based on the rows in table 110.

We suggest that the properties portion of our work might correlate with the group $S U(17)$.

Table 111 illustrates the notion that table 58 correlates with the group $S U(17)$ and with six applications of equation (47). This essay does not speculate regarding possibilities for detecting phenomena that would correlate with PR216ISP but not with PR36ISP.

Table 112 lists aspects that might support the notion that people might find a group theoretic expression that correlates with the list of elementary particles that nature includes. (Discussions regarding table 72 and table 75 might provide an alternative description relevant to some facets of the last three aspects that table 112 lists.)

Notions above may suffice to embrace much modeling of motion - including classical physics models and quantum physics models - that comports with six isomers of all elementary particles except Gfamily elementary particles, conservation of energy, conservation of momentum, and conservation of angular momentum. (Regarding six isomers of simple particles, see table 111a. The notion of six isomers of simple particles correlates with the notion that one might need to limit the range of applicability of modeling based on general relativity.) Notions above include quantum gravity - as an aspect of core proposed modeling PFS modeling and with independence from classical models (including general relativity) regarding motion. Classical modeling regarding motion correlates with ongoing modeling KMS modeling.

Beyond notions above, we are uncertain as to the extent to which people might want to add group theoretic concepts related to specific modeling regarding motion. (For example, people might treat Newtonian physics as comporting with special relativity in the limit of small velocities. If so, people might not want to add group theoretic concepts related to Newtonian physics.)

Notions above might suffice for people to state a group theoretic expression that correlates with the superset.

\subsection{The strong CP problem}

We discuss insight, that proposed modeling might provide, regarding the strong CP problem.

Ongoing modeling explores the possibility that the strong interaction contributes to violation of $\mathrm{CP}$ symmetry (or, charge conjugation parity symmetry). People might have yet to detect strong interaction contributions to the violation of CP symmetry. People use the three-element term strong CP problem. Unverified ongoing modeling suggests that such violation might correlate with the existence of axions.

Table 113 lists aspects correlating with insight that proposed modeling might provide regarding the extent to which nature includes strong CP violation. Table 113a lists aspects that people might interpret as ruling out strong interaction contributions to $\mathrm{CP}$ violation. Table $113 \mathrm{~b}$ lists aspects that, if relevant, people might interpret as suggesting that the strong interaction contributes to $\mathrm{CP}$ violation.

\subsection{Possible lacks of some unverified ongoing modeling elementary particles}

We discuss notions that proposed modeling correlates with nature possibly not including some elementary particles that some unverified ongoing modeling models suggest might exist.

\subsubsection{A possible lack of magnetic monopoles}

Table 92 points to no G-family solutions that would correlate with interactions with a magnetic monopole elementary particle. The lack of such G-family solutions might correlate with nature not including a magnetic monopole elementary particle. People might want to consider the notion that equation 177) expresses.

The 2G2 solution correlates with electromagnetic (not magnetic) monopole moments. 
Table 111: Possible correlation between properties and an $S U(17)$ symmetry

(a) Possible correlations between properties and subgroups of $S U(17)$

\begin{tabular}{|c|c|c|c|c|}
\hline$j_{1}+j_{2}$ & $j_{1}$ & $j_{2}$ & Use of $S U\left(j_{2}\right)$ & Use of $U(1)$ \\
\hline 17 & 15 & 2 & Charge & Conservation of charge \\
\hline 15 & 11 & 4 & Rest energy minus freeable energy & $\begin{array}{l}\text { Conservation of rest energy minus } \\
\text { freeable energy }\end{array}$ \\
\hline 11 & 9 & 2 & 3LB number & Conservation of 3LB number \\
\hline 9 & 7 & 2 & Momentum & Conservation of momentum \\
\hline 7 & 5 & 2 & Angular momentum & Conservation of angular momentum \\
\hline 5 & 3 & 2 & $\begin{array}{l}\text { Component of an } S U(2) \times U(1) \\
\text { symmetry }\end{array}$ & $\begin{array}{l}\text { Component of an } S U(2) \times U(1) \\
\text { symmetry }\end{array}$ \\
\hline 3 & - & 3 & $\begin{array}{l}S U(3) \text { strong interaction } \\
\text { symmetry }\end{array}$ & - \\
\hline
\end{tabular}

(b) Notes regarding table $111 \mathrm{a}$

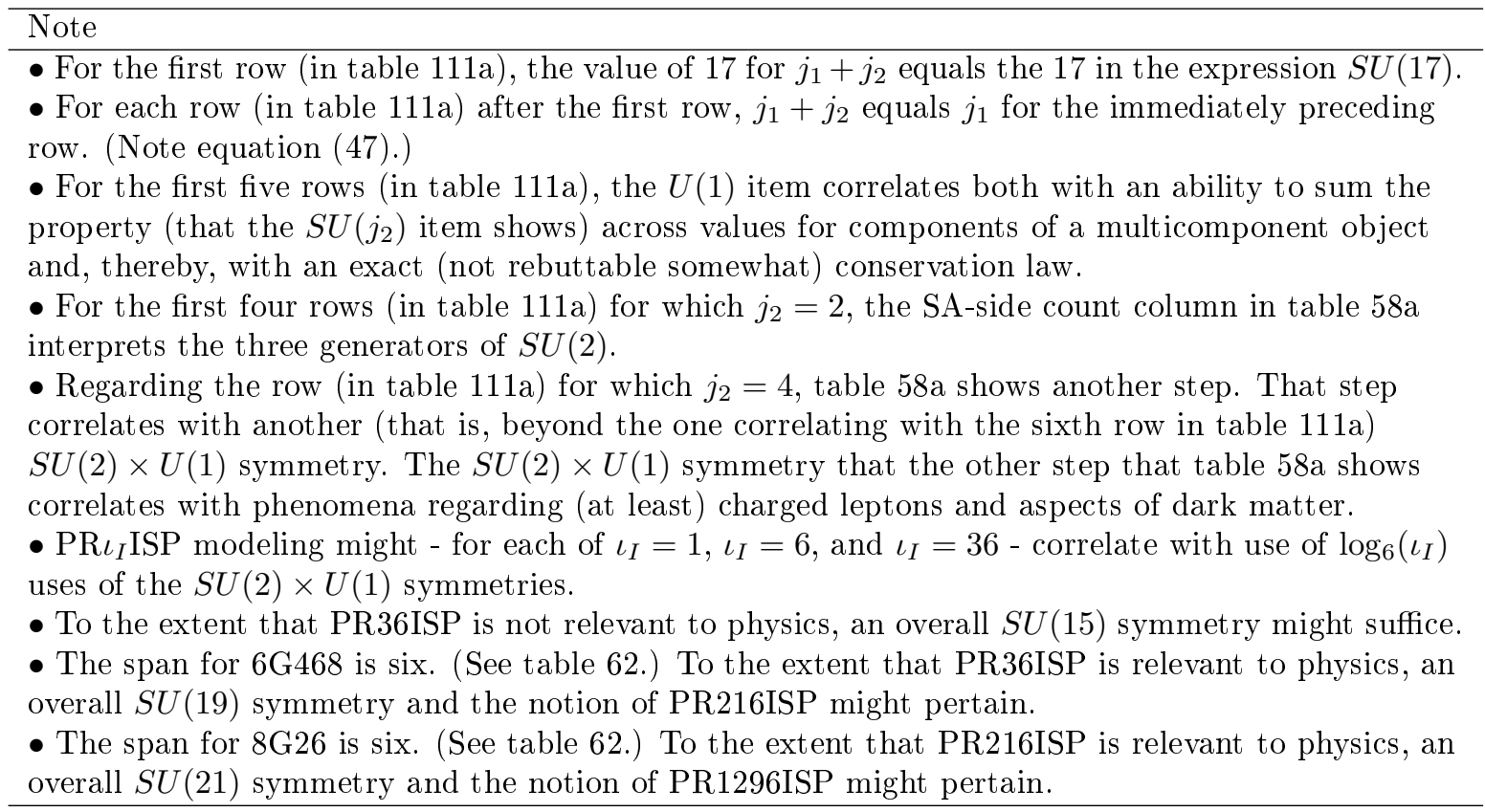

Table 112: Aspects that might support the notion that people might find a group theoretic expression that correlates with the list of elementary particles that nature includes

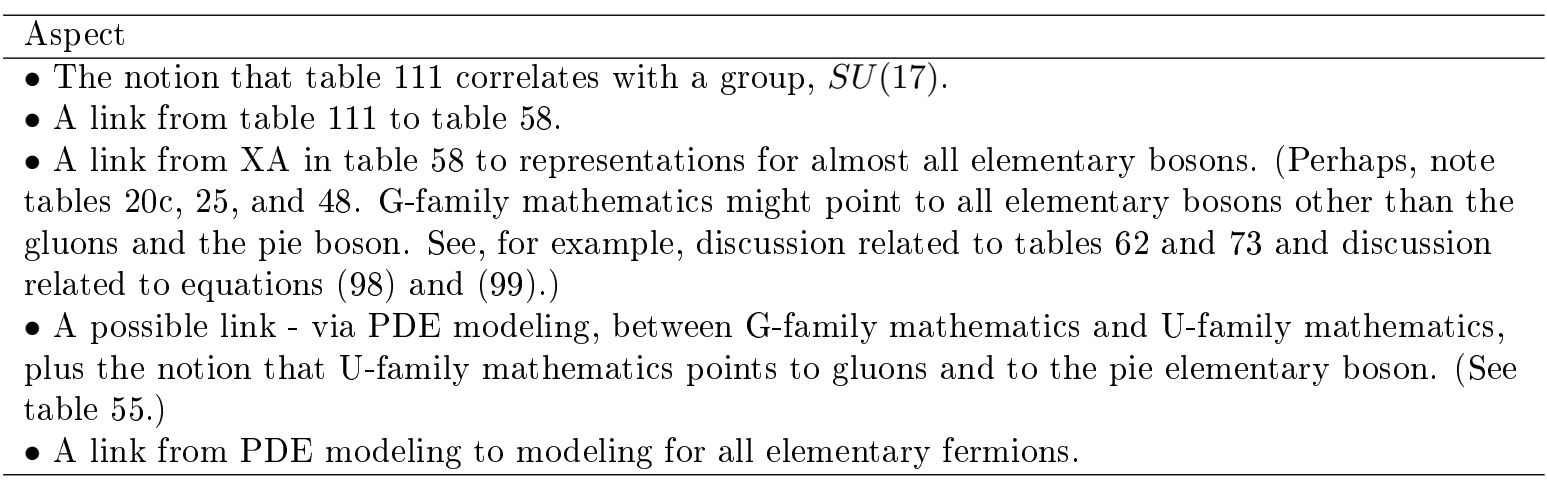


Table 113: Aspects correlating with insight that proposed modeling might provide regarding the extent to which nature includes strong CP violation

(a) Aspects that people might interpret as ruling out strong interaction contributions to CP violation

\begin{tabular}{|c|}
\hline \\
\hline 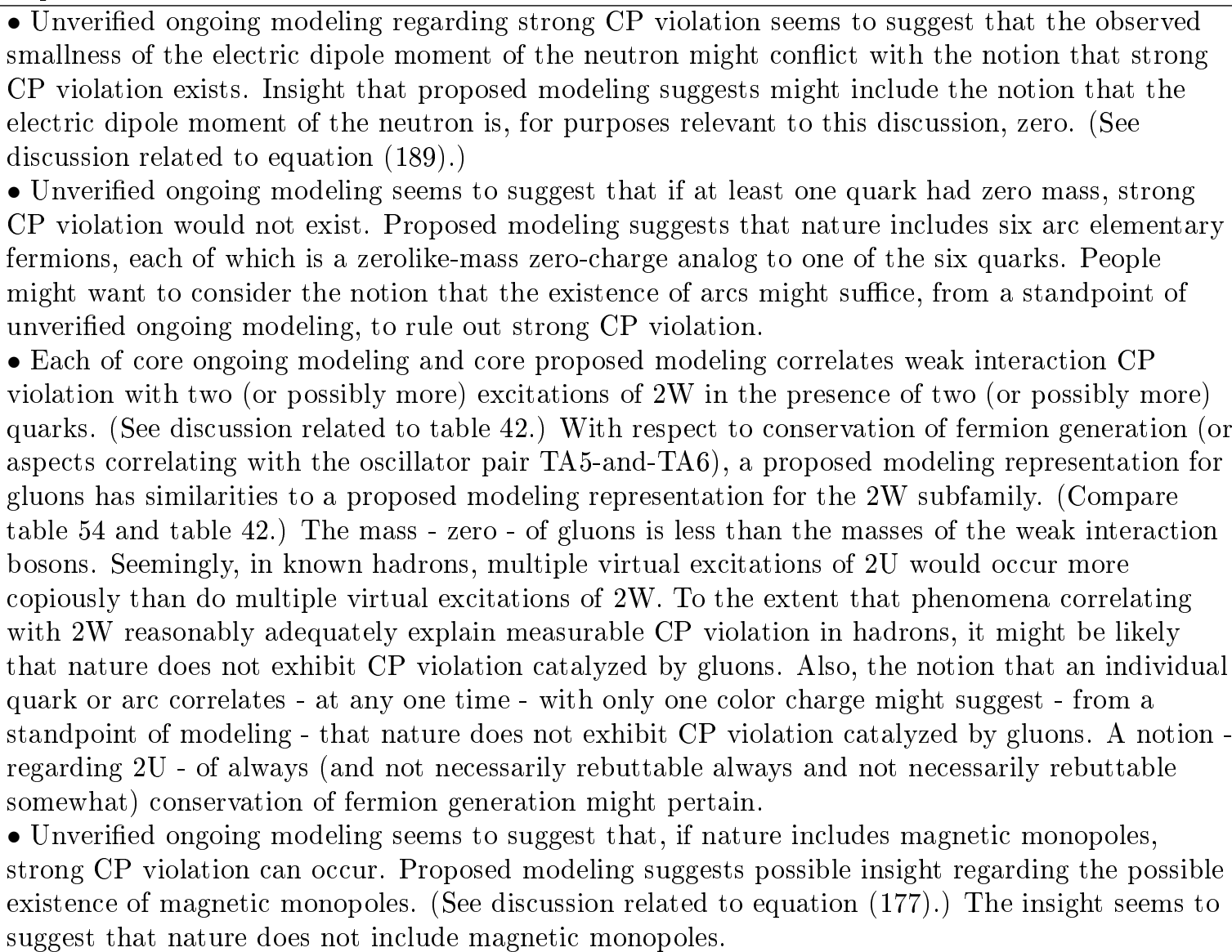 \\
\hline
\end{tabular}

(b) An aspect that, if relevant, people might interpret as suggesting that the strong interaction contributes to CP violation

\begin{tabular}{l}
\hline Aspect \\
- Detection of currently hypothetical so-called axion elementary particles might point to \\
non-conservation - that might correlate with the strong interaction - of CP symmetry. \\
\hline
\end{tabular}


Table 114: Comparative features of supplementary proposed modeling dynamics modeling and ongoing modeling dynamics modeling

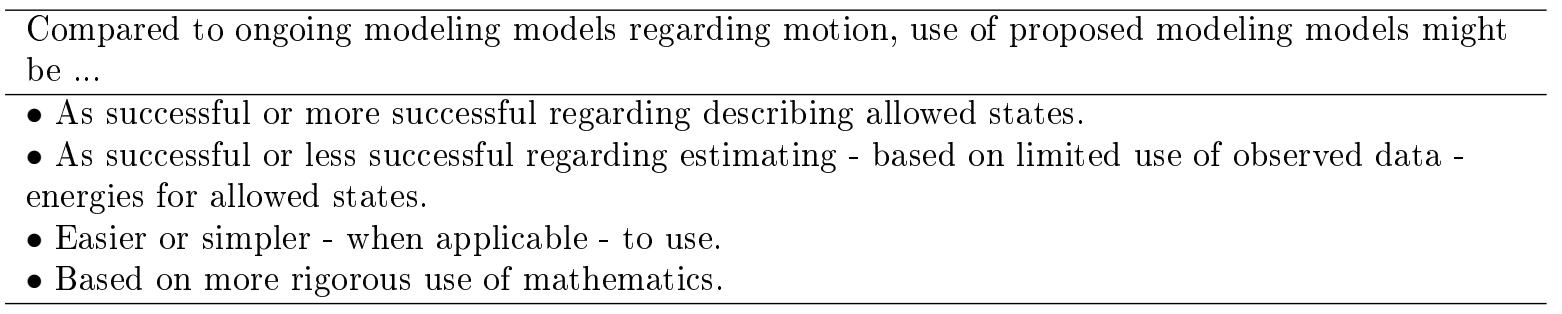

\subsubsection{A possible lack of axion elementary particles}

Each of the following statements might point to insight regarding attempting to detect axions. Proposed modeling suggests the possibility that people might mistake observations of phenomena related to the difference between 2(6)G248 and 2(1)G248 for observations related to axions. (See discussion related to equation (199).) Proposed modeling suggests the possibility that people might mistake observations of phenomena related to the aye (or, 0I) boson for observations related to axions. (For example, equation (166) shows an interaction that people might interpret as correlating with producing a magnetic field.)

\section{Discussion: supplementary proposed modeling and core ongoing modeling}

This unit explores the possibility that supplementary proposed modeling points to useful models for motion.

Discussion above in this essay features proposed modeling suggestions regarding elementary particles and dark matter particles, plus ongoing modeling models regarding motion. We generally assume that the PEPM particle set and ongoing modeling models for motion are adequately compatible with each other. We generally assume that the PEPM particle set and ongoing modeling models for motion are adequate for modeling relevant aspects of nature.

Discussion herein speculates about proposed modeling that would have bases in core proposed modeling models and would pertain directly to motion.

\subsection{Modeling that proposed modeling suggests regarding dynamics}

Table 30 alludes to possible proposed modeling applications, based on mathematics that underlies PDE modeling, to aspects of nature beyond the application correlating with matching known and suggesting new elementary particles.

We use the term CQFM to denote results from developing, from PFS modeling, a possibly useful somewhat analog to ongoing modeling QFT (or, quantum field theory). The acronym CQFM correlates with the four-word term complementary quantum field modeling. Ongoing modeling QFT modeling correlates with the notion of KMS modeling. CQFM would feature KMS modeling. (See table 17.)

We do not necessarily expect that proposed modeling models for motion duplicate ongoing modeling models for motion. Ongoing modeling models tend to be linear in energy. Ongoing modeling quantum models for motion tend to be linear in $\hbar$. Proposed modeling models for motion tend to be quadratic in energy. Proposed modeling quantum models tend to be quadratic in $\hbar$. (Note, for example, that $\Omega_{S A}$ in equation (4) can correlate with the expression $S(S+1) \hbar^{2}$.) Indeed, we anticipate that CQFM might include models that feature potentials or that CQFM might point to models that feature potentials.

We do not necessarily expect that proposed modeling aspects that seem to have parallels to ongoing modeling QFT (or, quantum field theory) need to comply with special relativity. (Regarding ongoing modeling, reference [85] discusses a definition of QFT that does not necessarily imply a correlation with special relativity.)

Table 114 compares aspects of supplementary proposed modeling dynamics modeling and aspects of ongoing modeling dynamics modeling.

\subsubsection{CQFM interaction vertices that involve simple particles and root forces}

We explore notions that underlie possible CQFM models regarding interaction vertices. (See the second row in table 30. Perhaps, see aspects, that mention $\nu_{S A}<0$, of table 31.)

This work generalizes from work above that, nominally, pertains for simple particles. Equations (53) and (54) pertain regarding all simple particles and all root forces. We posit that results - regarding some 
Table 115: Interaction vertices for interactions involving only simple particles and root forces (with $\nu$ denoting the effective $\nu)$

\begin{tabular}{lcl}
\hline Interaction & $\nu$ & Example \\
\hline $0 \mathrm{f}+1 \mathrm{~b} \leftrightarrow 2 \mathrm{f}+0 \mathrm{~b}$ & -1 & A Z boson creates a matter-and-antimatter pair of fermions. \\
$1 \mathrm{f}+1 \mathrm{~b} \leftrightarrow 1 \mathrm{f}+1 \mathrm{~b}$ & $-3 / 2$ & An electron and a $\mathrm{W}^{+3}$ boson produce a neutrino. \\
$1 \mathrm{f}+1 \mathrm{~b} \leftrightarrow 3 \mathrm{f}+0 \mathrm{~b}$ & $-3 / 2$ & A matter fermion and a boson produce two matter fermions and \\
& & one antimatter fermion. \\
$(3 \mathrm{f}+0 \mathrm{~b} \leftrightarrow 3 \mathrm{f}+0 \mathrm{~b})$ & $-3 / 2$ & - \\
$0 \mathrm{f}+\mathrm{nb} \leftrightarrow \mathrm{ff}+\mathrm{nb}$, for $\mathrm{n} \geq 2$ & $-n$ & A Higgs boson creates two photons. \\
\hline
\end{tabular}

roles for $\nu_{S A}, \nu_{T A}$, and $\nu^{\prime \prime}$ - from that work extend to all simple particles and all root forces. (See, for example, table 32b.)

Table 115 lists types of interaction vertices that proposed modeling includes. Here, in the symbol nf, $\mathrm{n}$ denotes a number of simple fermions. In the symbol $\mathrm{nb}, \mathrm{n}$ denotes a number of simple bosons and root forces. A symbol of the form $a \leftrightarrow b$ denotes two cases, namely $a \rightarrow b$ and $b \rightarrow a$. A symbol of the form $\mathrm{a} \rightarrow \mathrm{b}$ denotes the notion that the interaction de-excites each component of a by one unit and excites each component of b by one unit. (Note, for example, that de-excitation of a photon mode does not necessarily produce a ground state. Note, for example, that $1 \mathrm{~b}$ can correlate with $0 \mathrm{I}^{0}$, which is the aye boson.) For each type of interaction vertex, the effective $\nu$ is the sum, over incoming field solutions, of the relevant $\nu_{-}$and is also the sum, over outgoing field solutions, of the relevant $\nu_{-}$. In effect, the value of effective $\nu$ can correlate with aspects of a product of solutions of the form that equation (6) shows. The case $3 \mathrm{f}+0 \mathrm{~b} \leftrightarrow 3 \mathrm{f}+0 \mathrm{~b}$ pertains mathematically, but does not explicitly involve bosons. We are uncertain, in the current context, as to the possible relevance of $3 \mathbf{f}+0 \mathrm{~b} \leftrightarrow 3 \mathbf{f}+0 \mathrm{~b}$. In a broader context, $3 \mathbf{f}+0 \mathrm{~b} \leftrightarrow 3 \mathbf{f}+0 \mathrm{~b}$ might point toward possibilities for extending work herein.

CQFM posits that the notion of $3 \mathrm{f}$ does not necessarily violate ongoing modeling notions of fermion statistics. CQFM features aspects that appear to aggregate QFT interactions. (For one example, CQFM does not necessarily require notions of virtual particles. For this example, CQFM appears to aggregate multiple QFT Feynman diagrams. For another example, CQFM points toward modeling that replaces bosons with potentials.) Leaving aside the notion of aggregation of interactions, 3 f can involve dissimilar elementary fermions. Dissimilarity can correlate with differences regarding generations; matter and antimatter; and (if nothing else) types of simple particle - neutrino, charged lepton, quark, or arc.

We discuss an example that contrasts core ongoing modeling QFT and supplementary proposed modeling CQFM.

Proposed modeling can accommodate, for the weak interaction, modeling that does not require the notion of virtual particles. Equation (178) shows an ongoing modeling $1 \mathrm{f}+1 \mathrm{~b} \leftrightarrow 1 \mathrm{f}+1 \mathrm{~b}$ vertex. A muon transforms into a matter neutrino and a $\mathrm{W}$ boson. Equation (179) shows an ongoing modeling $1 \mathrm{f}+1 \mathrm{~b} \leftrightarrow 1 \mathrm{f}+1 \mathrm{~b}$ vertex. The $\mathrm{W}$ boson transforms into an electron and an antimatter neutrino. Core proposed modeling can accommodate that modeling. Supplementary proposed modeling can accommodate that modeling and can accommodate the $1 \mathrm{f}+1 \mathrm{~b} \leftrightarrow 3 \mathrm{f}+0 \mathrm{~b}$ vertex that equation $180 \mathrm{p}$ shows. Equation (180) does not show a virtual particle such as a W boson. Modeling based on equation (180) can be useful. However, modeling based just on equation (180) would not necessarily support research that estimates properties of the W boson and would not necessarily estimate the strength of the interaction that equation (180) shows.

$$
\begin{gathered}
\mu^{-3}+0 \mathrm{I}^{0} \rightarrow \nu^{0}+\mathrm{W}^{-3} \\
\mathrm{~W}^{-3} \rightarrow \mathrm{e}^{-3}+\bar{\nu}^{0} \\
\mu^{-3}+0 \mathrm{I}^{0} \rightarrow \nu^{0}+\mathrm{e}^{-3}+\bar{\nu}^{0}
\end{gathered}
$$

\subsubsection{Supplementary proposed modeling dynamics models re multicomponent objects}

We discuss the possibility that CQFM extends to include interactions involving objects that are not elementary particles.

For proposed modeling models of interactions that involve simple particles and root forces in free environments, the KMS PDE notion of the mathematical limit expression $\left(\eta_{S A}\right)^{2} \rightarrow 0$ pertains. (See discussion related to equation (13).) Here, $\left(\eta_{T A}\right)^{2} \rightarrow 0$ pertains. We say that the vertex models as being 
Table 116: PDE symbols and, for modeling related to physics dynamics, dimensions correlating with terms

\begin{tabular}{|c|c|c|c|}
\hline Symbol & Discussion & Dimensions - square of ... & Related constant \\
\hline$\xi_{S A}^{\prime}$ & $\xi_{S A}^{\prime} \Omega_{S A} \propto S(S+1) \hbar^{2}$ & Angular momentum & $\hbar^{2}$ \\
\hline$\xi_{S A}^{\prime}\left(\eta_{S A}\right)^{-2}$ & & Momentum & \\
\hline$\xi_{T A}^{\prime}\left(\eta_{T A}\right)^{-2}$ & & Energy & \\
\hline$\left(\eta_{S A}\right)^{2} /\left(\eta_{T A}\right)^{2}$ & & Velocity & $c^{2}$ \\
\hline$\xi_{S A}^{\prime}\left(\eta_{S A}\right)^{+2}$ & & Angular momentum times length & \\
\hline$\xi_{T A}^{\prime}\left(\eta_{T A}\right)^{+2}$ & & Energy times square of time & \\
\hline
\end{tabular}

point-like with respect to KMS coordinates. Here, point-like refers to the temporal coordinate and refers to either a radial spatial coordinate or three spatial coordinates.

An example of modeling of interactions that involve simple particles in so-called confined environments might feature modeling regarding interactions with a quark that exists within a proton.

For proposed modeling models of interactions that involve simple particles and root forces in confined environments, the PDE notion of $\left(\eta_{S A}\right)^{2}>0$ can pertain. The expression that equation 181 shows might correlate with the size of the multicomponent object that correlates with the term confined environment. We say that the vertex models as being volume-like with respect to coordinates. Here, volume-like refers to, at least, either a radial spatial coordinate or three spatial coordinates. Volume-like correlates also with a non-point-like domain for the temporal coordinate.

$$
\left|\eta_{S A}\right|
$$

We discuss some aspects of proposed modeling models. (These remarks tend to correlate with the last row in table 30. However, some of these remarks pertain regarding the existence of elementary particles and regarding the first row in table 116. See discussion regarding table 55 and the pie simple boson.)

Table 116 notes aspects of PDE mathematics that can pertain for dynamics modeling and $\nu_{S A} \geq 0$. In table 116, the associations that the first row shows provide a basis for the remaining rows. The row that notes $\xi_{S A}^{\prime}\left(\eta_{S A}\right)^{+2}$ might point to a series - momentum, angular momentum, and angular momentum times length.

PDE-based modeling might correlate with some aspects of unification of the strong, electromagnetic, and weak interactions. We consider modeling for which $2 \nu_{S A}$ is a non-negative integer. Based on the $r^{-2}$ spatial factor, the $V_{-2}$ term might correlate with the square of an electrostatic potential. (See table 8.) Based on the $r^{2}$ spatial factor, the $V_{+2}$ term might correlate (at least, within hadrons) with the square of a potential correlating with the strong interaction. The sum $K_{0 a}+K_{0 b}$ might correlate with the strength of the weak interaction. (The effective range of the weak interaction is much smaller than the size of a hadron. Perhaps, the spatial characterization $r^{0}$ correlates with an approximately even distribution, throughout a hadron, for the possibility of a weak interaction occurring.) Based on the $V_{-2}$ term, we expect that $\xi_{S A}^{\prime}$ includes a factor $\hbar^{2}$.

Electrostatics includes each of interactions that attract objects to each other and interactions that repel objects from each other. One might consider the possibility that, in some modeling, the term proportional to $\Omega_{S A} / r^{2}$ might seem to allow for repulsion, but not for attraction. (See equations (3) and (4). The term correlates with a contribution that is proportional to $+r^{-2}$. The potential decreases as $r$ increases.) However, when equations (31), 182, and 183 pertain, one can swap the $\Omega_{S A} / r^{2}$ term and the $\Omega_{T A} / t^{2}$ term in equation (31). The swap leads, in effect, to a new $\Omega_{S A} / r^{2}$ that has a sign that is opposite to the sign correlating with the old $\Omega_{S A} / r^{2}$. The new $\Omega_{S A} / r^{2}$ would correlate with attraction. For some aspects of modeling, equations $(184)$ and $(185)$ pertain.

$$
\begin{gathered}
\left(t / \eta_{T A}\right)^{2}=\left(r / \eta_{S A}\right)^{2} \\
\xi_{T A}^{\prime}=\xi_{S A}^{\prime} \\
t^{2} /\left(2\left(\eta_{T A}\right)^{2}\right)+r^{2} /\left(2\left(\eta_{S A}\right)^{2}\right)=\operatorname{tr} /\left(\left|\eta_{T A}\right| \cdot\left|\eta_{S A}\right|\right) \\
v_{c}=\left|\eta_{S A}\right| /\left|\eta_{T A}\right|
\end{gathered}
$$

We anticipate exploring notions correlating with the third and fourth rows in table 30 . 


\subsubsection{Dynamics models for hadron-like particles}

We discuss various topics regarding hadron-like particles.

We discuss the notion that each hadron-like particle that includes no more than three quarks (or, 1Q particles) and arcs (or, 1R particles) does not include both quarks and arcs.

Discussion related to table 49 suggests that a hadron-like particle has a charge for which the magnitude is either zero or a non-zero integer multiple of $\left|q_{\epsilon}\right|$ and has a baryon number that is either zero or a nonzero integer multiple of one. For a hadron-like particle that includes no more than three quarks and arcs, the restrictions to integer charge and integer baryon number preclude the simultaneous presence of more than zero quarks and more than zero arcs.

We discuss modeling for dynamics in hadrons that contain no more than three quarks.

Ongoing modeling QCD (or, quantum chromodynamics) modeling correlates with symmetries, for each of quarks and gluons, that correlate with special relativity.

We explore the notion that proposed modeling suggests possibilities for modeling that correlates, with each of quarks and gluons, a less than full set of symmetries correlating with special relativity.

Modeling for a free hadron requires two TA-side $S U(5)$ symmetries and four SA-side $S U(2)$ symmetries. (See discussion - regarding combining two objects to form one free object - related to table 60 . There, we assume that the original two objects are objects that can model as being free objects. Here, we do not assume that the original objects necessarily can model as being free. Here, we retain the notions of a set of kinematics symmetries for the motion of a combined object and a set of dynamics symmetries for internal aspects of the same combined object.) Proposed modeling suggests that each one of bosons (within the hadron) and simple fermions (within the hadron) can contribute one TA-side $S U(5)$ symmetry and two SA-side $S U(2)$ symmetries. One TA-side $S U(5)$ symmetry and two SA-side $S U(2)$ symmetries correlate with modeling for the free hadron. The other TA-side $S U(5)$ symmetry correlates with modeling for dynamics regarding internal aspects of the hadron. For each one of bosons and simple fermions, modeling might correlate with just one SA-side $S U(2)$ symmetry.

This proposed modeling dynamics modeling correlates with the notion that neither one of quarks and gluons behaves like free simple particles. Proposed modeling suggests that a hadron-like particle must include at least two (non-virtual) unfree fermions. (The notion of virtual correlates with ongoing modeling. Core proposed modeling can work in conjunction with modeling that includes the notion of virtual fermions and in conjunction with modeling that does not include the notion of virtual fermions.)

We discuss notions that might correlate with modeling that might output masses for hadrons.

References [86] and [87] suggest opportunities to improve understanding regarding modeling that might explain the masses of hadrons such as protons. Proposed modeling suggests concepts that might help regarding such opportunities. One concept correlates with avoiding relying on modeling that correlates with special relativity. (See discussion nearby above.) One concept correlates with equations (3) and (4) and with $D=3$. Here, the term that is proportional to $r^{2}$ might correlate with the square of a potential. For a two-quark hadron, the potential associated with one quark affects the other quark. For a three-quark hadron, the potential associated with two quarks affects the third quark.

We discuss modeling for dynamics in hadrons and hadron-like particles that contain more than three quarks.

Reference [88] discusses an observation of tetraquarks that feature four charm quarks, of which two are matter quarks and two are antimatter quarks. The article suggests that people might benefit by considering that such tetraquarks might feature two components. Each component would feature a matter charm quark and an antimatter charm quark. Proposed modeling suggests that the pie boson might correlate with the binding to each other of the two components.

Reference [89] suggests that some of the dynamics within at least some pentaquarks correlates with the dynamics for a system composed of a meson-like particle and a baryon-like particle. The meson-like particle features a matter quark and an antimatter quark. The baryon-like particle features three matter quarks. Aspects that proposed modeling correlates with the pie boson might play roles in such dynamics.

\subsubsection{Dynamics models for nuclear physics}

We discuss possibilities for developing proposed modeling models for atomic nuclei.

Ongoing modeling bases some aspects of modeling on notions of a Pauli exclusion force and on notions of a Yukawa potential. Ongoing modeling correlates these effects with notions of a residual strong force. The Pauli exclusion force keeps hadrons apart from each other. The Yukawa potential attracts hadrons to each other. Modeling suggests virtual pions as a source for the Yukawa potential.

Reference [90] expresses concerns regarding modeling some aspects of nuclear physics based on the notion of virtual pions. 
Core proposed modeling PFS modeling and core ongoing modeling KMS modeling can pertain. Here, KMS modeling includes a Pauli exclusion force and a notion of virtual pions.

Supplementary proposed modeling KMS modeling does not correlate with a Pauli exclusion force or with notions of virtual pions.

From a standpoint of modeling, pie (or, $0 \mathrm{P}$ ) bosons might correlate with attraction between hadrons. (See discussion related to table 55.) The attraction might correlate with a PDE-centric expression proportional to the term that equation (186) shows. (See discussions related to equations (181) and (184).)

$$
-\exp \left(-\operatorname{tr} /\left(\left|\eta_{T A}\right| \cdot\left|\eta_{S A}\right|\right)\right) \rightarrow-\exp \left(-r /\left(\left|\eta_{S A}\right|\right)\right)
$$

Proposed modeling suggests that jay (or, 2J) bosons correlate with repulsion between some pairs of hadrons that people know to occur in atomic nuclei. Each hadron in known atomic nuclei correlates with a $3 \mathrm{LB}$ number of minus one. Proposed modeling suggests that the $2 \mathbf{J}_{-}$boson correlates with the relevant repulsion. (See table 70 .)

We are uncertain as to the extent to which such models for atomic nuclei would improve on ongoing modeling techniques.

\subsubsection{Dynamics models for atomic physics}

We discuss possibilities for developing proposed modeling models for atomic physics.

Regarding some atomic physics, people might want to explore using modeling that correlates with equations (187) and (188). Equation (187) can correlate with de-emphasizing non-residual aspects of the strong force. The strong force is not relevant to the relevant aspects of atomic physics. KMS PDE modeling might feature electrons in a potential that correlates with an atomic nucleus and perhaps correlates with other electrons. Numbers of electrons per shell and per subshell can - based on two notions - correlate with numbers that nature exhibits. One notion features relevance of the Laplacian operator that equation (4) shows. One notion features a limit of no more than two electron spin states per solution correlating with the Laplacian operator. The numbers of electrons do not correlate with the existence of the $r^{2}$ term in equation (3).

$$
\begin{gathered}
\left(\xi_{S A}^{\prime} / 2\right)\left(\eta_{S A}\right)^{-2} \rightarrow 0^{+} \\
\left(\xi_{S A}^{\prime} / 2\right)\left(\eta_{S A}\right)^{2} \text { is a positive constant }
\end{gathered}
$$

\subsubsection{Dynamics models for quantum transitions}

We discuss the possibility that aspects of proposed modeling pertain to temporal aspects of quantum transitions.

People may have observed quantum transitions that take non-zero time. (See reference [91].)

Proposed modeling suggests the possibility that people can model such aspects of transitions via volume-like vertices. Modeling that features volume-like vertices might parallel temporal aspects of equation (186). (See discussions regarding equations (184) and (186).)

\subsection{Possible applications of proposed modeling KMS modeling}

We explore possible applications of supplementary proposed modeling KMS modeling.

\subsubsection{A possible lack of a neutron electric dipole moment}

We discuss modeling that would comport with the notion that nature does not include a non-zero neutron electric dipole moment.

Equation (189) shows an upper bound on the electric dipole moment for the neutron. (See reference [13]. Here, the one-letter symbol m denotes meters.)

$$
0.30 \times 10^{-27}\left|q_{\epsilon}\right| \mathrm{m}
$$

For each hadron for which dynamics modeling based on supplementary proposed modeling PDE techniques pertains and for which all the quarks occupy one state with respect to spatial characteristics, the electric dipole moment might be zero. (See discussion, related to table 8, regarding PDE-based modeling that correlates with some aspects of the strong, electromagnetic, and weak interactions.)

Proposed modeling suggests that the neutron and proton might be such hadrons. 
Some research suggests that some pentaquarks might not be such hadrons. (See interpretation, in reference [89], of reference [92].)

We think that this discussion comports with comparisons that table 114 suggests.

\subsubsection{Anomalous magnetic dipole moments}

We explore two possibilities regarding supplementary proposed modeling approaches to estimating charged lepton anomalous magnetic dipole moments.

Equations 190, (191), and 192 show ongoing modeling KMS interpretations of results of experiments regarding anomalous magnetic dipole moments. (See reference [13].) The subscripts $\epsilon$, $\mu$, and $\tau$ denote, respectively, electron, muon, and tauon. The symbol $a$ correlates with anomalous magnetic dipole moment. The symbol $\alpha$ denotes the fine-structure constant. (See equation (109).)

$$
\begin{gathered}
a_{\epsilon}-(\alpha /(2 \pi)) \approx-1.76 \times 10^{-6} \\
a_{\mu}-(\alpha /(2 \pi)) \approx+4.51 \times 10^{-6} \\
-0.052<a_{\tau}<+0.013
\end{gathered}
$$

Ongoing modeling provides means, correlating with Feynman diagrams, to calculate an anomalous magnetic dipole moment for each of, at least, the electron and the muon. The ongoing modeling Standard Model suggests computations whereby the anomalous magnetic dipole moment for a charged lepton is a sum of terms. The first term is $\alpha /(2 \pi)$. The second term is proportional to $\alpha^{2}$. The third term is proportional to $\alpha^{3}$. The exponent associated with $\alpha$ correlates with a number of virtual photons.

Regarding the tauon, equation (193) shows a result correlating with a first-order Standard Model (or, ongoing modeling) calculation. (See reference [93.)

$$
a_{\tau, \mathrm{SM}} \approx+1.177 \times 10^{-3}
$$

We explore a possible proposed modeling approach that tries to parallel the ongoing modeling approach.

Proposed modeling suggests that notions of anomalous electromagnetic moments correlate with $\gamma 2$ solutions. Electromagnetic dipole solutions correlate with $\gamma 2$ solutions for which RSDF is $r^{-3}$. The following remarks pertain for other than the $2 \mathrm{G} 24$ solution, which correlates with the ongoing modeling nominal magnetic moment result of $g \approx 2$. (2G24 correlates with $2 \gamma$ and not with $\gamma 2$.) The relevant solutions might be 4G26, 6G24, 6G28, 8G26, and 10G28. However, 6G28 and 10G28 do not interact with individual simple fermions. (Each of $6 \mathrm{G} 28$ and $10 \mathrm{G} 28$ correlates with a TA-side $S U(5)$ symmetry. See table 62. Perhaps, note table 93.) Solutions 6G28 and 10G28 might correlate with, for example, the Lamb shift. Regarding anomalous electromagnetic dipole moments, we assume that 4G26, 6G24, and $8 \mathrm{G} 26$ pertain.

We explore the possibility that proposed modeling suggests that contributions to $a$ scale as $\alpha^{(\Sigma-2) / 2}$. (Compare with discussion regarding equation (143).)

Solution 4G26 might correlate with the $\alpha /(2 \pi)$ term that ongoing modeling provides for charged leptons. For solution $6 \mathrm{G} 24,4 \in \Gamma$. Solution $6 \mathrm{G} 24$ might correlate with a result that varies with charged lepton rest mass. Solution 6 G24 might correlate with a term that is proportional to $\alpha^{2} /(2 \pi)$. (See equation (190), equation (191), and discussion regarding table 118 , Note the result $\alpha^{2} /(2 \pi) \approx 8.48 \times 10^{-6}$.) Solution 8G26 might correlate with a term proportional to $\alpha^{3} /(2 \pi)$.

We try to estimate $a_{\tau}$.

We assume that the $4 \mathrm{G} 26$ solution correlates with the ongoing modeling result of $\alpha /(2 \pi)$. We assume that the 6G24 solution correlates with contributions of the order $\alpha^{2}$.

We assume that, for a charged lepton $\mathrm{cl}$, equation 194 pertains. Here, $t_{\mathrm{cl}}$ is the construct that the first column of table 117 identifies.

$$
a_{\mathrm{cl}}-(\alpha /(2 \pi)) \approx a_{6 \mathrm{G} 24,1}+a_{6 \mathrm{G} 24, \mathrm{t}} t_{\mathrm{cl}}
$$

Table 117 shows approximate possible values for $a_{6 \mathrm{G} 24,1}$ and $a_{6 \mathrm{G} 24, \mathrm{t}}$, based on fitting data that equations (190) and (191) show and based on using various candidates for $t_{\mathrm{cl}}$. We de-emphasize the notion that $8 \mathrm{G} 26$ might also contribute to an actual value.

Table 118 provides, based on table 117 and equation (194), some possible suggestions for $a_{\tau}-(\alpha /(2 \pi))$. The comparison is with respect to a Standard Model first order calculation. (See equation (193).) Per 
Table 117: Possible approximations regarding the $6 \mathrm{G} 24,1$ and $6 \mathrm{G} 24, \mathrm{t}$ contributions to $a_{\mathrm{cl}}-(\alpha /(2 \pi))$ for charged leptons

\begin{tabular}{ccc}
\hline Assumption regarding $t_{\mathrm{cl}}$ & $a_{6 \mathrm{G} 24,1}$ & $a_{6 \mathrm{G} 24, \mathrm{t}}$ \\
\hline$m$ & $-1.79 \times 10^{-6}$ & $5.96 \times 10^{-8}$ \\
$m^{2}$ & $-1.76 \times 10^{-6}$ & $5.62 \times 10^{-10}$ \\
$M^{\prime \prime}$ & $-1.76 \times 10^{-6}$ & $3.13 \times 10^{-6}$ \\
$\left(M^{\prime \prime}\right)^{2}$ & $-1.76 \times 10^{-6}$ & $1.57 \times 10^{-6}$ \\
generation & $-8.03 \times 10^{-6}$ & $6.27 \times 10^{-6}$ \\
$(\text { generation })^{2}$ & $-3.85 \times 10^{-6}$ & $2.09 \times 10^{-6}$ \\
$\log \left(m / m_{\epsilon}\right)$ & $-1.76 \times 10^{-6}$ & $1.18 \times 10^{-6}$ \\
$\left(\log \left(m / m_{\epsilon}\right)\right)^{2}$ & $-1.76 \times 10^{-6}$ & $2.21 \times 10^{-7}$ \\
\hline
\end{tabular}

Table 118: Possible approximations for $a_{\tau}-(\alpha /(2 \pi))$

\begin{tabular}{ccccc}
\hline $\begin{array}{c}\text { Assumption } \\
\text { regarding first } \\
\text { order behavior } \\
\text { for }\end{array}$ & $\begin{array}{c}\text { First order } \\
\text { suggestion for } \\
a_{\tau}-(\alpha /(2 \pi))\end{array}$ & $\begin{array}{c}\text { Prediction for } \\
a_{\tau}\end{array}$ & $\begin{array}{c}\text { Approximate } \\
\text { comparison } \\
\left(a_{\tau}-a_{\tau, \mathrm{SM}}\right) / a_{\tau, \mathrm{SM}}\end{array}$ & Fit \\
$a_{\mathrm{cl}}-(\alpha /(2 \pi))$. & & & \\
The term is & & & \\
linear in a & & & & \\
lepton's: & & & & \\
\hline$m$ & $+1.04 \times 10^{2} \times 10^{-6}$ & $+1.266 \times 10^{-3}$ & $+75 \times 10^{-3}$ & - \\
$m^{2}$ & $+1.77 \times 10^{3} \times 10^{-6}$ & $+2.933 \times 10^{-3}$ & $+1500 \times 10^{-3}$ & - \\
$M^{\prime \prime}$ & $+7.65 \times 10^{-6}$ & $+1.169 \times 10^{-3}$ & $-6.9 \times 10^{-3}$ & $!$ \\
$\left(M^{\prime \prime}\right)^{2}$ & $+12.35 \times 10^{-6}$ & $+1.174 \times 10^{-3}$ & $-2.9 \times 10^{-3}$ & $!$ \\
generation & $+10.8 \times 10^{-6}$ & $+1.172 \times 10^{-3}$ & $-4.3 \times 10^{-3}$ & $!$ \\
$(\text { generation })^{2}$ & $+15.0 \times 10^{-6}$ & $+1.176 \times 10^{-3}$ & $-0.7 \times 10^{-3}$ & $! !$ \\
$\log \left(m / m_{\epsilon}\right)$ & $+7.83 \times 10^{-6}$ & $+1.169 \times 10^{-3}$ & $-6.8 \times 10^{-3}$ & $!$ \\
$\left(\log \left(m / m_{\epsilon}\right)\right)^{2}$ & $+12.9 \times 10^{-6}$ & $+1.174 \times 10^{-3}$ & $-2.5 \times 10^{-3}$ & $!$ \\
\hline
\end{tabular}

the notion that the interaction strength does not necessarily correlate linearly or quadratically with an ongoing modeling property and per the quadratic behavior with respect to $\left|q_{\epsilon}\right|$ in the expression $\alpha^{(\Sigma-2) / 2}$, appropriate results might correlate with the square of generation or with the square of a function of $\log (m)$. (See work that includes equation (108).)

Each one of the results that table 118 shows comports with experimental results. Except for the row regarding $m$ and the row regarding $m^{2}$, each row in table 118 might comport with the calculation based on the Standard Model. The (generation) ${ }^{2}$-centric result that table 118 shows might comport best, of the results that the table suggests, with the calculation based on the Standard Model. The (generation) $)^{2}$-centric result differs from the result that equation (193) shows by about 0.7 parts in 1000 .

Based on the notion that contributions to $a$ scale as $\alpha^{(\Sigma-2) / 2}$ and on results that table 117 shows, it might seem unlikely that $a_{6 \mathrm{G} 24,1}$ correlates with $8 \mathrm{G} 26$. However, it is possible that the strength of interactions correlating with $4 \mathrm{G} 26$ differs from the ongoing modeling result that correlates with $\alpha /(2 \pi)$ and that $a_{6 \mathrm{G} 24,1}$ correlates with such a difference.

Given remarks just above, we explore another approach to estimating $a_{\tau}$.

We assume that the strength of each of 4G26 and 8G26 does not change with generation. We assume that, in effect, equation 195 pertains. We assume that, in effect, equation (196) pertains. Here, we have assumed a clean split between contributions that do not correlate with generation and contributions that do correlate with generation. For the left side of equation (195), $4 \notin \Gamma$. For the left side of equation (196), $4 \in \Gamma$. Regarding table 118, the leftmost column and the rightmost three columns pertain regarding this approach. (Technically, one needs to change the column heading for the leftmost column. The new heading should be the following: "Assumption regarding the behavior for $a_{6 \mathrm{G} 24}$. The term is linear in a lepton's:".)

$$
\begin{gathered}
a_{4 \mathrm{G} 26}+a_{8 \mathrm{G} 26}=(\alpha /(2 \pi))+a_{6 \mathrm{G} 24,1} \\
a_{6 \mathrm{G} 24}=a_{6 \mathrm{G} 24, \mathrm{t}}
\end{gathered}
$$


Table 119: Possible correlations, regarding PR1ISP modeling, with general relativity

\begin{tabular}{ll}
\hline Aspect regarding proposed modeling & Aspect regarding general relativity \\
\hline $4 \mathrm{G} 48$ & rotational frame-dragging \\
$4 \mathrm{G} 48$ repulsion & Einstein field equations \\
$4 \mathrm{G} 246$ attraction & Einstein field equations \\
$4 \mathrm{G} 2468 \mathrm{a}$ and $4 \mathrm{G} 2468 \mathrm{~b}$ repulsion & Einstein field equations \\
$4 \mathrm{G}$ components other than $4 \gamma$ components & Einstein field equations \\
RSDF (or, radial spatial dependence of force) of $r^{-6}$ & Cosmological constant \\
\hline
\end{tabular}

Discussion related to equations (195) and (196) suggests the possibility that proposed modeling can pertain via modeling that features just two terms. One term would not vary with generation. One term would vary with generation.

Here, the following notions may correlate with each other. Modeling for freeable energy features oscillator pair SA5-and-SA6. The solution 6G24 correlates with $\Sigma=6$ and with oscillator pair SA5-andSA6. The notion that $t_{\mathrm{cl}}$ equals (generation) ${ }^{2}$ might pertain. (See, in table 118, the column labeled fit. For this example, the notion of freeable energy correlates with generation.) The exponent of two in the expression (generation) ${ }^{2}$ parallels the exponent of two that pertains regarding the factor $\left(q_{\epsilon}\right)^{2}$ in $\alpha$ in the sense that contributions seem to scale as the squares of particle properties.

We think that the second approach illustrates comparisons that table 114 suggests.

We also note that, regarding choices - that table 118 shows - regarding $t_{\mathrm{cl}}$ and ignoring the choices of $m$ and $m^{2}$, each of the three quadratic choices - $\left(M^{\prime \prime}\right)^{2}$, (generation) $)^{2}$, and $\left(\log \left(m / m_{\epsilon}\right)\right)^{2}-$ might be more appropriate than any of the three linear choices - $M^{\prime \prime}$, generation, and $\log \left(m / m_{\epsilon}\right)$. This essay de-emphasizes looking for deeper meaning regarding the role of aspects - such as this one regarding table 118 or similar ones regarding equation (133) - that pertain to squares of properties.

\subsection{Possibilities to complement ongoing modeling classical physics}

We explore possibilities that supplementary proposed modeling might offer useful complements to core ongoing modeling classical physics modeling.

\subsubsection{Possibilities for using Newtonian modeling in place of general relativity}

Table 119 lists aspects related to 4G $\Gamma$ solutions. In the context of PR1ISP modeling, each row (possibly except for the last row) in the table points to a possible correlation with general relativity. For each row, the extent to which the possible correlation pertains might be an open question. People associate the twoelement term Lense-Thirring effect with the two-element term rotational frame-dragging. The Einstein field equations allow solutions that correlate with repulsion. This essay does not explore the extent to which modeling based on the notion of an RSDF (or, radial spatial dependence of force) of $r^{-6}$ and on the notion of $\rho \neq 0$ might correlate with general relativity modeling for which a non-zero cosmological constant pertains. (See discussion related to table 62.)

People might explore the feasibility of developing supplementary proposed modeling KMS modeling based on correlations that table 119 suggests. People might explore the extent to which such supplementary proposed modeling KMS modeling can be useful regarding PR1ISP modeling and regarding PR6ISP modeling.

\subsubsection{Possibilities for extending aspects related to Maxwell's equations}

A standard representation of Maxwell's equations features two properties and two fields. The properties are charge and current. The fields are an electric field and a magnetic field.

People try to embed aspects of Maxwell's equations into broader contexts. People try to develop or understand models based on such broader contexts. People look for evidence that such models comport with nature.

Special relativity provides an example. Here, charge and current become components of a 4-vector.

The notion of a magnetic monopole provides another example. Here, people try adding a property to Maxwell's equations. People have yet to find evidence that nature includes magnetic monopoles. (See discussion related to equation (177).)

Our work suggests possibilities for another example. (See table 66.) Here, up to six properties might pertain. Three properties would be charge, nominal magnetic dipole moment, and rotating nominal magnetic dipole moment. Here, rotation is likely with respect to an axis that does not equal the axis that correlates with the nominal magnetic dipole moment. The three properties do not necessarily 
Table 120: Possible themes for experiments or observations

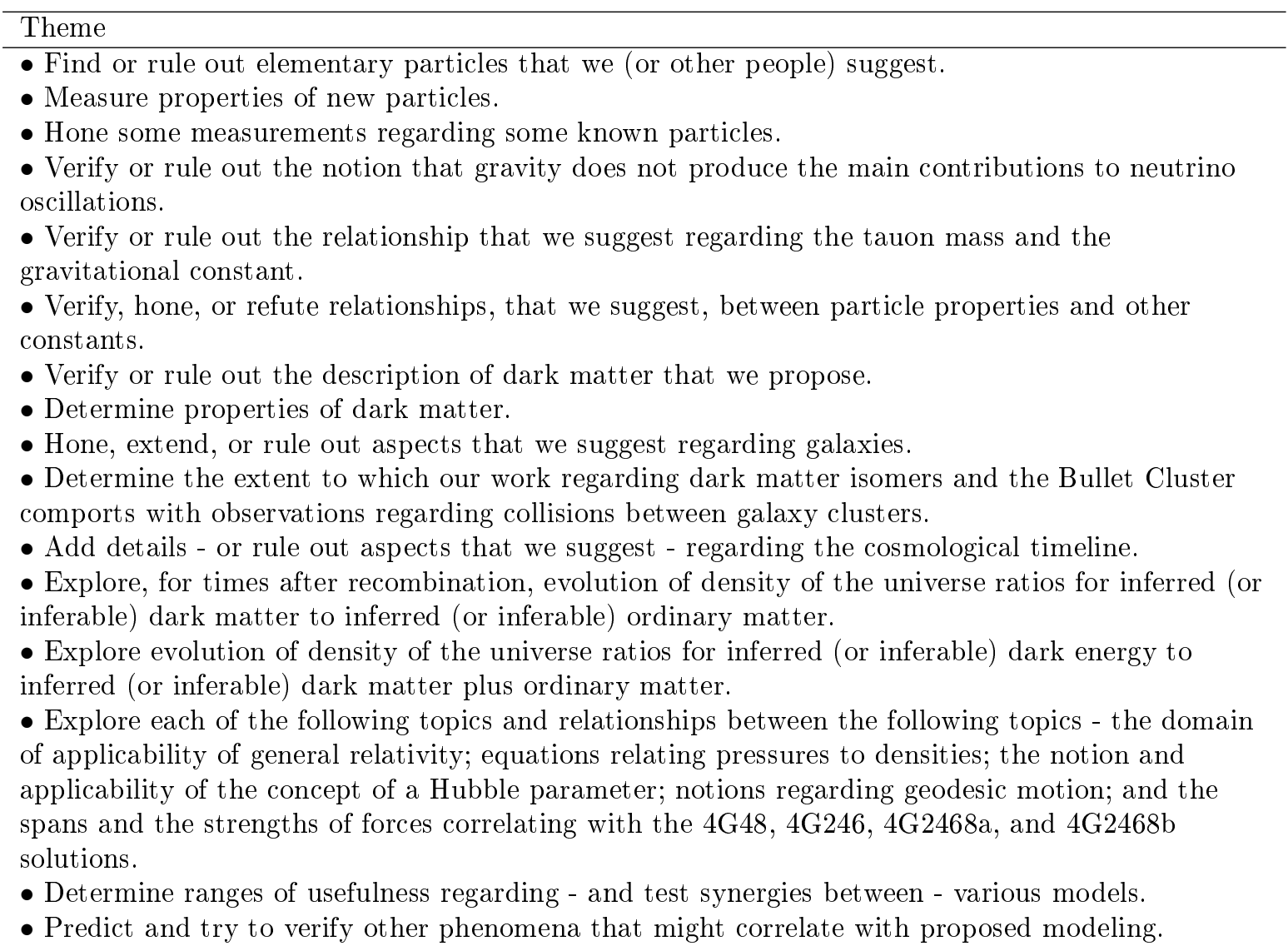

correlate with aspects of translational motion. The three properties correlate respectively with the words monopole, dipole, and quadrupole. The three properties correlate respectively with the terms scalar (or, rank-zero tensor), vector (or, rank-one tensor), and rank-two tensor. The other up to three properties would correlate with motion of the first three properties. For example, one property would be charge current, which correlates with the notion of moving charge.

Our work might suggest possibilities for yet another example. The previous example features three properties that do not necessarily correlate with translational motion. People might extend the previous example by considering the three-element series - which is related to translational motion - static, moving with an unchanging velocity, and moving with a changing velocity. Here, the notion of moving with a changing velocity might correlate with linear motion and acceleration and might correlate with angular velocity.

\section{Discussion: possible opportunities}

This unit notes possible opportunities for research.

\subsection{Possible opportunities for experimental or observational research}

We note possible opportunities for experimental or observational research.

\subsubsection{Possible themes for experiments or observations}

Table 120 suggest themes for experiments and observations that people might want to conduct. This essay de-emphasizes the topic of when techniques and technology will suffice to enable specific experiments or observations. We de-emphasize the topic of when - for each of various predictions we or other people make based on proposed modeling - falsifiability becomes feasible. 
Table 121: Possible roles for the aye particle and for the 0I solution and for the jay particles and 2J solutions

(a) Possible roles in nature for the aye particle and for the jay particles

Possible roles

- The 0I and 2J particles play roles before the inflationary epoch. (See discussion related to equation (163),)

- The 0I and 2J particles play roles during the inflationary epoch. (See discussion related to equation (164).)

- The 0I particle functions as the inflaton and plays a role after inflation. (See discussion related to equation (166).)

- The $0 \mathrm{I}$ and 2J particles help explain some interactions. (Note interactions to which table 70 alludes directly or indirectly.)

- The 2J particles help explain the ongoing modeling notion of the Pauli exclusion principle, some ongoing modeling aspects related to the ongoing modeling notion of the Pauli exclusion principle, and an observation - regarding positronium - that might point to a limitation regarding current ongoing modeling. (See table 70a.)

- The 0I and 2J particles help explain phenomena that ongoing modeling correlates with terms such as vacuum energy, vacuum fluctuations, or quintessence.

- The 0I and 2J particles explain phenomena that ongoing modeling correlates with density of dark energy. (See discussion related to equation (172).)

- The 0I particle might correlate with situation-specific interaction rates. (See discussion related to equation (197).)

(b) Possible roles in modeling for the 0I solution and for the jay solutions

Possible roles

- The 0I and 2J solutions help explain scaling by factors of $\alpha$ correlating with adding vertices or with increasing G-family spin. (See discussion related to equation (137).)

- The 0I and 2J solutions simplify some aspects of modeling (and, regarding such, do not necessarily correlate with nature).

\subsubsection{Possibilities for detecting or inferring aye and jay bosons}

Table 121 lists possible roles for the aye particle and for the 0I solution and for the jay particles and 2J solutions. Each item in table 121a might point to possibilities for detecting or inferring at least one of aye bosons and jay bosons. For example, the item regarding positronium might correlate - regarding jay bosons - with such a detection or inference.

We discuss some items - that table 121a shows - for which we can offer information to which table 121a does not point.

Some aspects of ongoing modeling propose interactions that would produce unspecified particles that people might not have detected. For example, people propose an interaction $K_{L}^{0} \rightarrow \pi^{0}+X$ for which there is an intermediate state of two simple fermions that interact via a $\mathrm{W}$ boson and produce the so-designated X particle. (See reference [94.) Here, the symbol $K_{L}^{0}$ correlates with the K-long meson. The symbol $\pi^{0}$ denotes a zero-charge pion. To the extent that this interaction actually occurs, proposed modeling suggests the possibility that the $\mathrm{X}$ particle is an aye boson or a jay boson.

Ongoing modeling proposes concepts such as interactions with a so-called quantum vacuum. Proposed modeling can be compatible with modeling that features a quantum vacuum and can be compatible with modeling that does not embrace a notion of quantum vacuum. Interactions with $0 \mathrm{I}$ and $2 \mathrm{~J}$ bosons might correlate with effects similar to effects that ongoing modeling correlates with the notion of interactions with a quantum vacuum.

Equation (197) shows a possibility for decay of a Higgs boson. The equation might correlate with a rate that is not very situation specific. (Here, we assume a lack of lasing.) Equation (198) shows another possibility for the decay of the Higgs boson. The equation might correlate with a rate that correlates with a density of $0 \mathrm{I}$ particles and might be situation specific.

$$
\begin{gathered}
\mathrm{OH}^{0} \rightarrow \ldots \\
\mathrm{OI}^{0}+\mathrm{OH}^{0} \rightarrow \ldots
\end{gathered}
$$

We discuss aspects that table $121 \mathrm{~b}$ shows. 
Discussion related to the relative strengths of some components of G-family forces points to terms proportional to $\alpha^{(\Sigma-2) / 2}$. (See discussion related to equation (137) and discussion related to equation (143).) Possibly, modeling based on the 0I solution and the 2J solutions correlates - in ways that this essay does not discuss - with aspects regarding spins, interactions, and KMS models. (See discussion related to equation (136).)

\subsubsection{Possibilities for directly detecting dark matter}

We discuss possibilities for observing dark matter effects without creating dark matter.

We discuss possibilities for inferring the presence of dark matter in seemingly ordinary matter neutron stars.

People might want to consider possibilities for inferring the presence of dark matter content in neutron stars. (See discussion related to equations (170) and (171).)

We discuss other possibilities for observing dark matter effects without creating dark matter.

Possibly, people can develop techniques for detecting gravitationally the presence of nearby dark matter.

People attempt to directly detect dark matter. (See, for example, reference 95.) Some efforts look for WIMPs. We are uncertain as to the extent to which these efforts might be able to detect $1 \mathrm{R} \otimes 2 \mathrm{U}$ hadron-like particles. Some efforts look for axions. We are uncertain as to the extent to which these efforts might attribute axion sightings to effects that correlate with the difference that equation (199) shows. Reference [96] discusses two attempts to detect dark matter bosons that would correlate with interactions between neutrons in ordinary matter atomic nuclei and electrons in ordinary matter atoms that include the atomic nuclei. (Reference [96] cites reference [97] as exemplifying experiments regarding isotopes of ytterbium and reference [98, as exemplifying experiments regarding isotopes of calcium.) Proposed modeling suggests that at least one of equation (199) and 2(2)G68 $\neq 2(1) \mathrm{G} 68$ might explain the types of effects that both attempts report.

$$
2(6) \mathrm{G} 248 \neq 2(1) \mathrm{G} 248
$$

Proposed modeling suggests new possibilities for directly detecting dark matter or doubly dark matter. To the extent that PR6ISP pertains to nature and PR36ISP does not pertain to nature, the following discussion pertains to detecting dark matter. To the extent that PR36ISP pertains to nature, the following discussion pertains to detecting doubly dark matter. The basis for one possibility is the difference between 2(6)G248 and 2(1)G248. Here, a detector might feature a rotating magnetic dipole moment, with the axis of rotation not matching (and perhaps being orthogonal to) the axis correlating with the magnetic dipole. (We are uncertain as to the extent that people might want to try to use, in the context of 2(6)G248, detectors based on magnons. See reference [99]. The reference discusses possibilities for detecting light dark matter and not necessarily the types of dark matter that proposed modeling suggests. However, the proposed detection would be via magnetic fields.) Independent of that possible means for detection, people might try to infer 2(6)G248 phenomena correlating with dark matter magnetic fields (or - for the PR36ISP case - 2(6)G248 phenomena correlating with doubly dark matter magnetic fields). A basis for another possibility is the difference between 2(2)G68 and 2(1)G68. Proposed modeling suggests that $2 \mathrm{G} 68$ correlates with, at least, some atomic transitions.

We discuss three possibilities for making and detecting dark matter.

Equations (200), 201), and 202 show interactions that would convert a neutron into a dark matter $1 \mathrm{R} \otimes 2 \mathrm{U}$ hadron-like particle that features three arc (or, 1R) simple fermions. (A neutron includes two $\mathrm{Q}^{-1}$ quarks and one $\mathrm{Q}^{+2}$ quark.) The minimum energy to trigger this set of interactions correlates with the sum of the rest energies of one neutron and two charged tweaks. A minimum range for that minimum energy is $417 \mathrm{GeV}$ to $427 \mathrm{GeV}$. (Here, we assume results that equations (98) and (99) show.) For an experiment, the number of conversions might be small. The following notions might correlate with such smallness. The range of the $2 \mathrm{~T}^{ \pm}$boson might be small compared to the size of a neutron. (See discussion related to equation (88).) Effects that ongoing modeling correlates with the two-word term Pauli exclusion might imply that the probability for the original three quarks to be adequately close to each other is low.

$$
\begin{gathered}
2\left(\mathrm{Q}^{-1} \rightarrow \mathrm{R}^{0}+\mathrm{T}^{-1}\right) \\
\mathrm{Q}^{+2}+\mathrm{T}^{-1} \rightarrow \mathrm{Q}^{+1}
\end{gathered}
$$




$$
\mathrm{Q}^{+1}+\mathrm{T}^{-1} \rightarrow \mathrm{R}^{0}
$$

We speculate about means for detecting such a conversion of a neutron into a three-arc hadron-like particle. We assume that the neutron resides in an atomic nucleus in a target material. Given the relevant energies, we assume that the three-arc particle exits the target. We speculate that people would not detect the three-arc particle. With one target and enough conversions that do not produce escapes of atomic nuclei, people might detect a change in the isotopic composition of the target. Possibly, an easiest detection would correlate with effects other than those we just mentioned. Such effects might correlate with byproducts of the interactions.

Equations (203), 204), and 205 show interactions that would convert a proton into a dark matter $1 \mathrm{R} \otimes 2 \mathrm{U}$ hadron-like particle that features three arc (or, $1 \mathrm{R}$ ) simple fermions. (A proton includes two $\mathrm{Q}^{+2}$ quarks and one $\mathrm{Q}^{-1}$ quark.) The minimum energy to trigger this set of interactions correlates with the sum of the rest energies of one proton and three charged tweaks. A minimum range for that minimum energy is $625 \mathrm{GeV}$ to $640 \mathrm{GeV}$. (Here, we assume results that equations (98) and (99) show.)

$$
\begin{gathered}
2\left(\mathrm{Q}^{+2} \rightarrow \mathrm{R}^{0}+\mathrm{T}^{+2}\right) \\
\mathrm{Q}^{-1} \rightarrow \mathrm{R}^{0}+\mathrm{T}^{-1} \\
2\left(\mathrm{~T}^{+2}\right)+\mathrm{T}^{-1} \rightarrow \mathrm{W}^{+3}+\mathrm{I}^{0}
\end{gathered}
$$

Compared with trying to detect the conversion of a neutron into dark matter, the possibility for converting a proton offers advantages and disadvantages. One advantage might be the possibility for detecting the weak interaction that the $\mathrm{W}^{+3}$ boson would catalyze. Another advantage might correlate with an ability to use colliding beams instead of an approach that might feature one beam and a fixed target. One disadvantage might be the need to use higher energy for the incoming particles.

Equations (206) and (207) show interactions that would convert a positron and an electron into the fermion components for a $1 \mathrm{R} \otimes 2 \mathrm{U}$ hadron-like particle that would have some similarity to a neutral pion. A threshold energy could be about $81 \mathrm{GeV}$. Detecting the $1 \mathrm{R} \otimes 2 \mathrm{U}$ particle might prove difficult. To the extent that the preferred decay of the particle features a matter neutrino and an antimatter neutrino, detecting decay products might be possible and prove difficult.

$$
\begin{aligned}
& \mathrm{C}^{+3} \rightarrow \mathrm{R}^{0}+\mathrm{W}^{+3} \\
& \mathrm{C}^{-3}+\mathrm{W}^{+3} \rightarrow \mathrm{R}^{0}
\end{aligned}
$$

\subsection{Possible opportunities regarding harmonic oscillator mathematics}

We note possible opportunities to explore or extend some aspects of harmonic oscillator mathematics and some aspects of modeling based on harmonic oscillator mathematics.

\subsubsection{Possible opportunities re PDE harmonic oscillator mathematics}

Discussion above includes - regarding $\Psi$ - the topic of normalization. (See discussion related to equation (12).) Discussion above does not include the topic of orthogonality. To the extent that people want to address orthogonality, people might want to add emphasis (compared to the emphasis in work above) to the aspect of angular coordinates. Generally, this essay de-emphasizes the topic of orthogonality.

People might want to explore possible opportunities - regarding mathematics or modeling - related to the transformation - regarding numbers of dimensions - that correlates with equation (15). We note bases for some possible opportunities. Possibly, people can find additional significance regarding the array of numbers for which table 9 shows a portion. Possibly, people can find significance regarding a set of similar arrays for which $j$ varies. 


\subsubsection{Possible opportunities re ALG harmonic oscillator mathematics and modeling}

Discussion above suggests using ALG modeling that is based on non-isotropic harmonic oscillators to model aspects regarding refraction. (See discussion related to table 68.) People might further explore using ALG modeling based on non-isotropic harmonic oscillator mathematics to do PFS modeling or to do KMS modeling.

People might try to express, via harmonic oscillator mathematics or combinations of symmetries, kinematics models or dynamics models that pertain for each of a few or many interacting particles or objects. People might try to develop parallels to ongoing modeling equations that, for example, sum momenta. (Discussion above points to symmetries that correlate with summable quantities related, for example, to charge, to lepton number minus baryon number, and to momenta. See, for example, table 58a.)

People might try to correlate modeling involving many objects with modeling regarding entropy. (See discussion related to equation (215).)

\subsection{Possible opportunities to develop deeper insight}

We discuss modeling that people might use as bases for developing new aspects of physics modeling.

\subsubsection{Numbers of dimensions}

Proposed modeling suggests that, at least in some sense, a number - three - of spatial dimensions correlates with $D_{S A}^{*}=3$ and a number - one - of temporal dimensions correlates with $D_{T A}^{*}=1$. (See equations (53) and (54).)

Proposed modeling includes modeling that features other than three spatial dimensions. (See, for example, the SA-side aspects of representations that table 48 shows or the column labeled with the oneelement symbol $D$ in table 32b.) Ongoing modeling includes modeling that features other than three spatial dimensions.

Some proposed modeling uses of notions of $D_{S A}^{*}=3$ and $D_{T A}^{*}=1$ include modeling that correlates with $\nu_{S A}<0$ and that outputs a list of known and possible elementary particles. (See table 30.) As far as we know, ongoing modeling does not include parallels to such aspects of proposed modeling. Ongoing modeling aspects that correlate with three spatial dimensions and one temporal dimension tend to correlate with proposed modeling aspects for which $\nu_{S A} \geq 0$ pertains. (See table 30.)

Equations (53) and (54) might provide a characterization that can be useful, for much physics modeling, regarding the notions of three spatial dimensions and one temporal dimension.

\subsubsection{Arrow of time}

Equation (184) and discussion related to equation (37) suggest a notion of a $\Psi(t, r)$ that correlates with the TA0-and-SA0 oscillator pair. (See equation (6).) We suggest that equation (208) might pertain. (Perhaps, see also discussion related to equation (184) and discussion related to equation (186).) The domains $t>0$ and $r>0$ pertain for $\Psi(t, r)$. Without loss of generality, we posit that $\eta_{T A}>0$ pertains regarding after an interaction, $\eta_{T A}>0$ does not pertain regarding before an interaction, $\eta_{T A}<0$ pertains regarding before an interaction, and $\eta_{T A}<0$ does not pertain regarding after an interaction. We posit that $\eta_{S A}>0$ pertains regarding elementary particles that exit an interaction, $\eta_{S A}>0$ does not pertain regarding elementary particles that enter an interaction, $\eta_{S A}<0$ pertains regarding elementary particles that enter an interaction, and $\eta_{S A}<0$ does not pertain regarding elementary particles that exit an interaction. Of the four possibilities $\eta_{T A}>0$ and $\eta_{S A}>0, \eta_{T A}<0$ and $\eta_{S A}<0, \eta_{T A}>0$ and $\eta_{S A}<0$, and $\eta_{T A}<0$ and $\eta_{S A}>0$, mathematically, $\Psi$ normalizes for only the first two possibilities.

$$
\Psi(t, r) \propto \exp \left(-t r /\left(\eta_{T A} \eta_{S A}\right)\right)
$$

To the extent that this modeling correlates with the topic of arrow of time, the lack of dual normalization regarding each of the case of incoming and the case of outgoing might provide insight.

The proposed modeling notion that aspects of modeling of conservation of energy correlate with an $S U(5)$ symmetry (and not necessarily with an ongoing modeling notion of $S 1 G$ symmetry) might provide insight regarding the topic of arrow of time. Proposed modeling tends to correlate $S U\left(\_\right)$symmetries with origins (with respect to coordinates) and with radial coordinates. 
Table 122: Possible correlations between some types of models and three notions of mass (with HO3-4 denoting that oscillator pairs of the form XA3-and-XA4 pertain)

\begin{tabular}{llcc}
\hline Models & Theme (regarding models) & $m$ & HO3-4 \\
\hline Core ongoing modeling KMS & Motions of objects & $m_{i n}$ & - \\
Core proposed modeling PFS & Interactions of objects with environments & $m_{p g}$ & Yes \\
Core proposed modeling UMS & Additive properties of objects & $m_{a g}$ & Yes \\
Supplementary proposed modeling KMS & Motions of objects & $m_{\text {in }}$ & Yes \\
\hline
\end{tabular}

\subsubsection{Inertial mass and gravitational mass}

We discuss the possibility that proposed modeling provides insight regarding the possible equivalence of inertial mass and passive gravitational mass.

We assume that ongoing modeling Newtonian mechanics pertains. Equation (209) describes the acceleration $\vec{a}$ of an object of inertial mass $m_{i n}$ because of a force $\vec{F}$. Equation 210 describes the force that an object of passive gravitational mass $m_{p g}$ experiences because of an object - of active gravitational mass $m_{a g}$ - that is located a distance $\vec{r}$ from the object correlating with $m_{p g}$. (To some extent, regarding $\vec{r}$, we assume that we can model all objects as being point-like.) The three-word term passive gravitational mass refers to a mass that correlates with reactions of an object to externally generated gravitational fields. The three-word term active gravitational mass refers to a mass that correlates with the gravitational field that correlates with an object (or, that the object generates). Also, $r=|\vec{r}|$. The notion that modeling based on equation (211) seems to pertain for objects has been a topic of discussion for centuries.

$$
\begin{gathered}
\vec{F}=m_{i n} \vec{a} \\
\vec{F}=\left(G_{N} m_{p g} m_{a g} / r^{2}\right) \cdot(-\vec{r} / r) \\
m_{i n}=m_{p g}
\end{gathered}
$$

For purposes of this discussion, we posit that one can assume that freeable energy equals zero. (See, for example, tables 58 and 111.)

Table 122 shows possible correlations between some types of models and three notions of mass. For some rows in table 122, oscillator pairs of the form XA3-and-XA4 pertain.

Aspects related to table 122 might suggest insight regarding possible equivalences - at least for purposes of modeling - among the three notions of mass.

\subsubsection{Notions that might link physics constants and modeling}

Table 123 shows speculation about possible conflations regarding two notions. One notion is the $\Sigma$ in G-family mathematical solutions $\Sigma G \Gamma$. One notion is quantities (or, properties) with which some $\Sigma \gamma$ components of G-family forces interact. Each quantity (or, property) might pertain for each of some aspects of classical physics modeling and some aspects of quantum physics modeling. (Compare with table 66 and table 84.) In table 123, an item in parentheses shows a non-zero magnitude that pertains for modeling that correlates with the notion of free environment. Except for regarding speed, the number is a minimal non-zero magnitude. (For charge, for unfree environments, $\left|q_{\epsilon}\right| / 3$ pertains. For 3LB, for unfree environments, the number one pertains. Except for regarding speed, the numbers are minimal nonzero magnitudes.) Some modeling regarding refraction and effective mass might correlate (via, aspects correlating with longitudinal polarization) with a lack of a minimal non-zero quantity. (See discussion related to equations (78) and (79).) Regarding the case of $\Sigma=16$, there might be a correlation with the notion that modeling might correlate boost symmetry with the oscillator pair SA15-and-SA16. (See discussion that includes discussion of table 60.) Such a correlation might be useful with solutions that allow $\lambda=\llbracket 16 \rrbracket \in \Gamma$.

Some items in table 123 might correlate, in essence, with other physics constants. Charge might correlate with $1 /\left(4 \pi \varepsilon_{0}\right)$ and the vacuum electric permittivity $\varepsilon_{0}$. Magnetic flux correlates with $\left|q_{\epsilon}\right|$ and $\hbar$ and might correlate with $\mu_{0}$, the vacuum magnetic permeability. Mass might correlate with $G_{N}$, the gravitational constant.

Proposed modeling might suggest opportunities to further explore relationships between charge and mass and relationships between strengths of components of G-family forces. For example, table 83 points to possible relationships between charge and mass. 
Table 123: Possible conflations regarding G-family solutions and properties with which G-family forces interact (with (_) denoting a suggested smallest non-zero property magnitude, _, regarding modeling objects in free environments; and with $\left(\left(\_\right)\right.$denoting a different type of non-zero physics constant)

\begin{tabular}{ccccc}
\hline$\Sigma$ & Scalar & Vector & 2-tensor & 3-tensor \\
\hline 0 & & & \\
2 & Charge $\left(\left|q_{\epsilon}\right|\right)$ & Magnetic flux $\left(K_{\mathrm{J}}\right)$ & $\begin{array}{c}\text { Precessing flux } \\
\text { Moments of inertia }\end{array}$ & Rotating moments \\
4 & Mass & Rotating mass & Spin $(\hbar / 2)$ & $?$ \\
6 & Freeable energy & & \\
8 & 3LB $(3)$ & & \\
$\ldots$ & & & \\
16 & Speed $((c))$ & & \\
\hline
\end{tabular}

Proposed modeling might offer an opportunity for new looks, regarding models, at relationships between handedness, chirality, helicity, lepton number or baryon number, rotation, and spin. (Note the row, in table 123 , for which $\Sigma=8$ pertains.)

Proposed modeling might suggest another opportunity to explore modeling related to masses. We discuss a possibly useful notion regarding masses of non-zero-mass simple particles. Equations (212) and (213) pertain. The symbol $m$ denotes mass. Boson simple particle masses tend to feature relationships regarding squares of masses. Equation (212) points to results that feature squares of masses. For each simple boson other than the pie and tweak bosons, the equation evaluates approximately to an integer. The equation might correlate with the 2 U-related potential that scales like $r^{1}$ and that pertains regarding quarks and arcs in hadron-like particles. Equation (213) points to results that feature logarithms of masses. For each simple fermion other than the neutrinos and the arcs, the equation evaluates somewhat approximately to an integer. The equation might correlate with $\Sigma$ G-related potentials that scale like $r^{-1}$ and that pertain regarding simple fermions that have quantum interactions with $2 \mathrm{G} 2$ and $4 \mathrm{G} 4$ root forces. Equation (214) follows from equation (213) and produces results pertaining to squares of simple fermion masses other than neutrino masses and arc masses.

$$
\begin{gathered}
\int_{0}^{m /\left(m_{Z} / 3\right)} 2 r^{1} d r \\
\int_{1}^{m / m_{\epsilon}} r^{-1} d r \\
\int_{1}^{m / m_{\epsilon}} 2 r^{-1} d r
\end{gathered}
$$

We are uncertain regarding the usefulness of further pursuing notions that we discuss immediately above.

\subsubsection{Entropy}

We consider cases of multicomponent objects that involve $k+1$ peer component objects. Here, $k$ is a nonnegative integer.

We consider the case of $k=1$. The multicomponent object includes two peer component objects. Compared with dynamics symmetries for the multicomponent object, the two peer components collectively contribute one too many instance of each of conservation of energy symmetry, conservation of angular momentum symmetry, and conservation of momentum symmetry. Modeling might re-assign the extra three symmetries to a combination of the two peer components and a field - such as a gravitational field - that correlates with interactions between the peer components.

We consider the case of $k>1$. Here, we de-emphasize the possibility of stepwise subdivision. An example of stepwise subdivision involves the sun, earth, and moon. For this example of stepwise subdivision, one might use two steps, each correlating with $k=1$. The first step considers each of the sun and the earth plus moon to be objects. The second step considers the earth plus moon to be a multicomponent object consisting of the earth and the moon. Without adequately significant additions to modeling, this example might correlate with modeling for which - regarding ocean tides - effects of lunar gravity pertain and effects of solar gravity do not pertain. 
For $k>1$ and no stepwise subdivision, ongoing modeling models become more complex than ongoing modeling models for two-body (or, $k=1$ ) systems. Many applications might pertain - for example, to astrophysical systems, to ideal gasses, and so forth. For some applications, keeping the number of fields at one might correlate with a notion of entropy and, at least within that notion, with the ongoing modeling expression for entropy that equation (215) shows. Here, people might want to consider at least one of the two cases $j=k+1$ and $j=k$. Here, people might want to consider each of a notion of entropy for physical systems and a notion that might correlate, regarding mathematics-based modeling, with a term correlating with the word entropy.

$$
j \log (j)
$$

\section{Concluding remarks}

This unit discusses possible opportunities based on proposed modeling.

Proposed modeling might provide impetus for people to tackle broad agendas that our work suggests. Proposed modeling might provide means to fulfill aspects of such agendas. Proposed modeling might fulfill aspects of such agendas.

Opportunities might exist to develop more sophisticated modeling than the modeling that we present. Such a new level of work might provide more insight than we provide.

Proposed modeling might suggest applied mathematics techniques that have uses other than uses that we make.

Proposed modeling might suggest - directly or indirectly - opportunities for observational research, experimental research, development of precision measuring techniques and data analysis techniques, numerical simulations, and theoretical research regarding elementary particle physics, nuclear physics, atomic physics, astrophysics, and cosmology.

\section{Acknowledgments}

William Lama provided useful comments regarding the effectiveness of drafts that led to parts of this essay.

The following people pointed, via presentations or writings, to topics or aspects that we considered for inclusion in the scope of the work: Alex Filippenko, Brian Greene, Robert McGehee, Risa Wechsler, and various science journalists.

The following people pointed, via personal contact, to topics or aspects that we considered for inclusion in the scope of the work: Andrea Albert, Raphael Bousso, Lance Dixon, Persis Drell, Kamal Melek Hanna, Wick Haxton, Nick Hutzler, William Lama, Surhud More, Holger Muller, J. Xavier Prochaska, Martin Rees, and Harrison Rose.

The following people helped formally publish aspects leading to work that this essay describes: Charles K. Chui, Kamal Melek Hanna, Keith Jones, and Zeger Karssen. The following people provided or pointed to aspects regarding expressing or propagating the work: Elliott Bloom, Man Ho Chan, Maxwell Chertok, Charles K. Chui, Andrei Lucian Dragoi, Steven Frautschi, Carl Frederick, Richard B. Holmes, Frank Hiroshi Ling, Michael Mulhearn, Richard A. Muller, Stephen Perrenod, Paul Preuss, Amir Sharif, and Wendy Shi. Various people involved in journal publishing pointed to aspects regarding expressing or propagating the work. The following people suggested perspective, means, or suggestions regarding people with whom to try to have discussions: Vint Cerf, Yanbei Chen, James S. Clegg, Bill Daul, Jiggs Davis, George Djorgovski, Erica Ellingson, Ron Fredericks, Vesselin Gueorguiev, Tucker Hiatt, William Lama, Lianne La Reine, Robert Morgan, Doug Osheroff, Kennan Salinero, Jim Spohrer, Peter Walstrom, and Jon F. Wilkins. The following people provided means or encouragement relevant to this work: various family members, Hugh E. DeWitt, George Michael, and various teachers.

Table 124 lists communications for which the following two sentences pertain. This essay cites the communications. We did not necessarily find information sufficient to qualify the communications for inclusion in the bibliography. 
Table 124: Communications that this essay cites but that do not necessarily qualify for inclusion in the bibliography

(a)

\begin{tabular}{ll}
\hline Communication & Note \\
\hline Asgari, M.; Lin, C.-A.; Joachimi, B. \& others. KiDS-1000 & We did not find a \\
Cosmology: Cosmic shear constraints and comparison between two & publication beyond the \\
point statistics. Link(2020): https://arxiv.org/abs/2007.15633 & stage of a preprint \\
& possibly submitted to \\
& Astronomy \& \\
& Astrophysics. \\
\hline
\end{tabular}

(b)

\begin{tabular}{ll}
\hline Communication & Note \\
\hline Clavin, W. Rotating Galaxies Galore 2020. Posted by the California & We did not find a name \\
Institute of Technology. Link(2020): & for a series of articles \\
https://www.caltech.edu/about/news/rotating-galaxies-galore & that pertains to this \\
& article. \\
\hline
\end{tabular}

(c)

\begin{tabular}{ll}
\hline Communication & Note \\
\hline LeFevre, O.; Bethermin, M.; Faisst, A. \& others. The & We found a claim of a \\
ALPINE-ALMA [CII] survey: Survey strategy, observations and & status of "working \\
sample properties of 118 star-forming galaxies at $4<\mathbf{z}<6.2019$. & paper." \\
Link(2020): https://arxiv.org/abs/1910.09517 & \\
\hline
\end{tabular}

(d)

\begin{tabular}{ll}
\hline Communication & Note \\
\hline Anonymous. Content of the universe - pie chart. National & We did not find a source \\
Aeronautics and Aerospace Administration, April 2013. Link(2020): & for the data that the \\
https://map.gsfc.nasa.gov/media/080998/index.html & chart presents. \\
\hline
\end{tabular}

(e)

\begin{tabular}{ll}
\hline Communication & Note \\
\hline Wong, K. C.; Suyu, S. H.; Chen, G. C.-F. \& others. H0LiCOW XIII. & We were unable to \\
A $2.4 \%$ measurement of $\mathrm{H}_{0}$ from lensed quasars: $5.3 \sigma$ tension & verify a claim of a status \\
between early and late-Universe probes. Mon. Not. R. Astron. Soc., & of "in press." \\
in press 2020. Link(2020): https://arxiv.org/abs/1907.04869 &
\end{tabular}

\section{References}

[1] Richard P. Feynman. Cargo Cult Science. Commencement address, California Institute of Technology, June 1974. Link(2020): http://calteches.library.caltech.edu/51/2/CargoCult.htm. 1

[2] Frank Wilczek. Could Einstein Get Published Today? The Wall Street Journal, CCLXXVI(74):C4, September 2020. Link(2020): https://www.wsj.com/articles/could-einstein-get-published-today11600974323. 1

[3] Eric Weisstein. Delta Function. Wolfram MathWorld web page. Link(2020): http://mathworld.wolfram.com/DeltaFunction.html. 2.2

[4] Kevin Harnett. Mathematicians Explore Mirror Link Between Two Geometric Worlds. Quanta Magazine, April 2018. Link(2020): https://www.quantamagazine.org/mathematicians-explore-mirrorlink-between-two-geometric-worlds-20180409/. 2.4

[5] D. Mendeleev. Ueber die beziehungen der eigneschaften zu den atomgewichten der elemente. Zeitschrift fur Chemie, 12:405-406, March 1869. 3.2, 3.2.2

[6] Julian Heeck. Unbroken B-L symmetry. Phys. Lett. B, 739:256-262, December 2014. 3.2.7 
[7] Francesco Renga. Experimental searches for muon decays beyond the Standard Model. Rev. Phys., 4:100029, November 2019. 3.2.7

[8] Sarah Charley. W bosons remain left-handed. Symmetry, July $2015 . \quad$ Link(2020): https://www.symmetrymagazine.org/article/july-2015/w-bosons-remain-left-handed. 3.2.7

[9] K. Abe, R. Akutsu, A. Ali, et al. Constraint on the matter-antimatter symmetry-violating phase in neutrino oscillations. Nature, 580(7803):339-344, April 2020. 3.2.7

[10] Viviana Niro and Pedro Machado. Sterile Neutrino Down but Not Completely Out. Physics, 13, August 2020. Link(2020): https://physics.aps.org/articles/pdf/10.1103/Physics.13.123. 4.2.4

[11] L. Gurung, T. J. Babij, S. D. Hogan, and D. B. Cassidy. Precision Microwave Spectroscopy of the Positronium $n=2$ Fine Structure. Phys. Rev. Lett., 125:073002, August 2020. 4.2.7, 6.4

[12] Matteo Rini. A Fine Positronium Puzzle. Physics, 13, August 2020. Link(2020): https://physics.aps.org/articles/v13/s99. 4.2.7

[13] M. Tanabashi and others (Particle Data Group). Review of Particle Physics. Phys. Rev. D, 98(3):030001, August 2018. 4.3.1, 4.3.3, 4.3.5, 4.3.5 4.3.5, 4.3.6, 4.3.12, 4.3.13, 5.3.2, 5.3.2, 5.3.18, $6.5,8.2 .1,8.2 .2$

[14] M. Tanabashi and others (Particle Data Group). Review of Particle Physics. Phys. Rev. D, 98(3):030001, August 2018 and update 2019. Link(2020): http://pdg.lbl.gov/index.html. 4.3.4, $4.3 .4,4.3 .5,4.3 .5,4.3 .6$

[15] Nathalie Palanque-Delabrouille, Christophe Yeche, Julien Baur, et al. Neutrino masses and cosmology with Lyman-alpha forest power spectrum. J. Cosmol. Astropart. Phys., 2015(11):011-011, November 2015. 4.3.12

[16] M. Aker, K. Altenmuller, M. Arenz, et al. Improved Upper Limit on the Neutrino Mass from a Direct Kinematic Method by KATRIN. Phys. Rev. Lett., 123(22), November 2019. 4.3.14

[17] G. Drexlin. Direct neutrino mass measurement. TAUP 2019, September 2019. Link(2020): http://www-kam2.icrr.u-tokyo.ac.jp/indico/event/3/contribution/27. 4.3.14

[18] Johanna L. Miller. Closing in on neutrino CP violation. Phys. Today, 2020(1):0423a, April 2020. 4.3.16, 5.3.8

[19] V. C. Rubin. Galaxy dynamics and the mass density of the universe. Proceedings of the National Academy of Sciences, 90(11):4814-4821, June 1993. 5.2.1

[20] Edward Witten. Cosmic separation of phases. Phys. Rev. D, 30(2):272-285, July 1984. 5.2.1

[21] M. H. Chan. Observational evidence for dark matter interacting through a Yukawa potential. Astrophys. J. Letters, 769(1):L2, May 2013. 5.2.1

[22] Motoi Endo, Koichi Hamaguchi, and Go Mishima. Constraints on hidden photon models from electron $g-2$ and hydrogen spectroscopy. Phys. Rev. D, 86(9):095029, Nov 2012. 5.2.1

[23] Ned Wright. Brief History of the Universe. UCLA web page, July 2004. Link(2020):

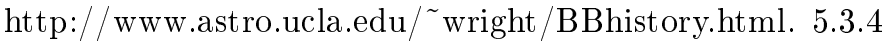

[24] Judd D. Bowman, Alan E. E. Rogers, Raul A. Monsalve, et al. An absorption profile centred at 78 megahertz in the sky-averaged spectrum. Nature, 555(7694):67-70, March 2018. 5.3.5

[25] Rennan Barkana. Possible interaction between baryons and dark-matter particles revealed by the first stars. Nature, 555(7694):71-74, March 2018. 5.3.5

[26] Paolo Panci. 21-cm line Anomaly: A brief Status. In 33rd Rencontres de Physique de La Vallee d'Aoste, July 2019. 5.3.5

[27] N. G. Busca, T. Delubac, J. Rich, et al. Baryon acoustic oscillations in the Lya forest of BOSS quasars. A\&A, 552(A96), April 2013. 5.3.6 
[28] S. Perlmutter, G. Aldering, G. Goldhaber, et al. Measurements of $\Omega$ and $\Lambda$ from 42 high-redshift supernovae $\Omega$. Astrophys. J., 517(2):565-586, June 1999. 5.3.6

[29] Adam G. Riess, Alexei V. Filippenko, Peter Challis, et al. Observational Evidence from Supernovae for an Accelerating Universe and a Cosmological Constant. Astron. J., 116(3):1009-1038, September 1998. 5.3.6

[30] Adam G. Riess, Louis-Gregory Strolger, John Tonry, et al. Type Ia Supernova Discoveries at z $>1$ from the Hubble Space Telescope: Evidence for Past Deceleration and Constraints on Dark Energy Evolution. Astrophys. J., 607(2):665-687, June 2004. 5.3.6

[31] Mark P. Hertzberg. Structure Formation in the Very Early Universe. Physics Magazine, 13(26), February 2020. Link(2020): https://physics.aps.org/articles/v13/16. 5.3.7, 5.3.7, 5.3.7

[32] Brian Green. Until the End of Time: Mind, Matter, and Our Search for Meaning in an Evolving Universe. Alfred A. Knopf, February 2020. 5.3.7, 5.3.7

[33] Charlie Wood. A New Cosmic Tension: The Universe Might Be Too Thin. Quanta Magazine, September 2020. Link(2020): https://www.quantamagazine.org/a-new-cosmic-tension-the-universemight-be-too-thin-20200908/. 5.3.10

[34] Khaled Said, Matthew Colless, Christina Magoulas, et al. Joint analysis of 6dFGS and SDSS peculiar velocities for the growth rate of cosmic structure and tests of gravity. Mon. Not. R. Astron. Soc., 497(1):1275-1293, July 2020. 5.3.10

[35] Supranta S. Boruah, Michael J. Hudson, and Guilhem Lavaux. Cosmic flows in the nearby Universe: new peculiar velocities from SNe and cosmological constraints. Mon. Not. R. Astron. Soc., August 2020. 5.3 .10

[36] Christopher Wanjek. Dark Matter Appears to be a Smooth Operator. Mercury, 49(3):10-11, October 2020. 5.3.10, 6.3

[37] Ewa L. Lokas and Gary A. Mamon. Dark matter distribution in the Coma cluster from galaxy kinematics: breaking the mass-anisotropy degeneracy. Mon. Not. R. Astron. Soc., 343(2):401-412, August 2003. 5.3.11

[38] Elena Rasia, Giuseppe Tormen, and Lauro Moscardini. A dynamical model for the distribution of dark matter and gas in galaxy clusters. Mon. Not. R. Astron. Soc., 351(1):237-252, June 2004. 5.3 .11

[39] Lawrence Rudnick. The Stormy Life of Galaxy Clusters: astro version. January 2019. Link(2020): https://ned.ipac.caltech.edu/level5/March19/Rudnick/frames.html. 5.3.11

[40] Lawrence Rudnick. The stormy life of galaxy clusters. Phys. Today, 72(1):46-52, January 2019. 5.3 .11

[41] Man Ho Chan. A tight correlation between the enclosed gravitational mass and hot gas mass in galaxy clusters at intermediate radii. Phys. Dark Universe, 28:100478, May 2020. 5.3.11

[42] Peter Behroozi, Risa Wechsler, Andrew Hearin, and Charlie Conroy. UniverseMachine: The correlation between galaxy growth and dark matter halo assembly from $\mathrm{z}=0-10$. Mon. Not. R. Astron. Soc., 488(3):3143-3194, May 2019. 5.3.14

[43] Nick Gnedin. Cosmological Calculator for the Flat Universe, 2015. Link(2020): http://home.fnal.gov/ gnedin/cc/. 5.3.14

[44] R. Genzel, N. M. Forster Schreiber, H. Ubler, et al. Strongly baryon-dominated disk galaxies at the peak of galaxy formation ten billion years ago. Nature, 543(7645):397-401, March 2017. 5.3.14

[45] J. Jimenez-Vicente, E. Mediavilla, C. S. Kochanek, and J. A. Munoz. Dark Matter Mass Fraction in Lens Galaxies: New Estimates from Microlensing. Astrophys. J., 799(2):149, January 2015. 5.3.14

[46] J. Jimenez-Vicente, E. Mediavilla, J. A. Munoz, and C. S. Kochanek. A ROBUST DETERMINATION OF THE SIZE OF QUASAR ACCRETION DISKS USING GRAVITATIONAL MICROLENSING. Astrophys. J., 751(2):106, May 2012. 5.3.14 
[47] Pieter van Dokkum, Roberto Abraham, Jean Brodie, et al. A High Stellar Velocity Dispersion and 100 Globular Clusters for the Ultra-diffuse Galaxy Dragonfly 44. Astrophys. J., 828(1):L6, August 2016. 5.3.14

[48] Shannon Hall. Ghost galaxy is 99.99 per cent dark matter with almost no stars. New Sci., August 2016. Link(2020): https://www.newscientist.com/article/2102584-ghost-galaxy-is-99-99-percent-dark-matter-with-almost-no-stars /. 5.3.14

[49] Pavel E. Mancera Pina, Filippo Fraternali, Elizabeth A. K. Adams, et al. Off the Baryonic Tully-Fisher Relation: A Population of Baryon-dominated Ultra-diffuse Galaxies. Astrophys. J., 883(2):L33, September 2019. 5.3.14

[50] Qi Guo, Huijie Hu, Zheng Zheng, et al. Further evidence for a population of dark-matter-deficient dwarf galaxies. Nature Astronomy, 4(3):246-251, November 2019. 5.3.14

[51] Pieter van Dokkum, Shany Danieli, Yotam Cohen, et al. A galaxy lacking dark matter. Nature, 555(7698):629-632, March 2018. 5.3.14

[52] Ignacio Trujillo, Michael A. Beasley, Alejandro Borlaff, et al. A distance of 13 Mpc resolves the claimed anomalies of the galaxy lacking dark matter. Mon. Not. R. Astron. Soc., 486(1):1192-1219, March 2019. 5.3.14

[53] Pieter van Dokkum, Shany Danieli, Roberto Abraham, et al. A Second Galaxy Missing Dark Matter in the NGC 1052 Group. Astrophys. J., 874(1):L5, March 2019. 5.3.14

[54] David A. Buote and Aaron J. Barth. The Extremely High Dark Matter Halo Concentration of the Relic Compact Elliptical Galaxy Mrk 1216. Astrophys. J., 877(2):91, May 2019. 5.3.14

[55] Ben Forrest, Marianna Annunziatella, Gillian Wilson, et al. An Extremely Massive Quiescent Galaxy at $\mathrm{z}=3.493$ : Evidence of Insufficiently Rapid Quenching Mechanisms in Theoretical Models. Astrophys. J., 890(1):L1, February 2020. 5.3.14

[56] Marcel Neeleman, J. Xavier Prochaska, Nissim Kanekar, and Marc Rafelski. A cold, massive, rotating disk galaxy 1.5 billion years after the Big Bang. Nature, 581(7808):269-272, May 2020. 5.3.14

[57] Adam S. Bolton, Tommaso Treu, Léon V. E. Koopmans, et al. The Sloan Lens ACS Survey. VII. Elliptical Galaxy Scaling Laws from Direct Observational Mass Measurements. Astrophys. J., 684(1):248-259, September 2008. 5.3.14

[58] T. Wang, C. Schreiber, D. Elbaz, et al. A dominant population of optically invisible massive galaxies in the early Universe. Nature, 572(7768):211-214, August 2019. 5.3.14

[59] Heather Hill. Massive galaxies from the early universe found hiding in plain sight. Phys. Today, September 2019. 5.3.14

[60] Laura V. Sales, Julio F. Navarro, Louis Penafiel, et al. The Formation of Ultra-Diffuse Galaxies in Clusters. Mon. Not. R. Astron. Soc., 494(2):1848-1858, March 2020. 5.3.14

[61] Massimo Meneghetti, Guido Davoli, Pietro Bergamini, et al. An excess of small-scale gravitational lenses observed in galaxy clusters. Science, 369(6509):1347-1351, September 2020. 5.3.15

[62] Maria Temming. Dark matter clumps in galaxy clusters bend light surprisingly well. Sci. News, September 2020. $\operatorname{Link}(2020)$ : https://www.sciencenews.org/article/dark-matter-clumps-galaxyclusters-bend-light-surprisingly-well. 5.3.15

[63] Duncan A Forbes, Adebusola Alabi, Aaron J Romanowsky, et al. Globular clusters in Coma cluster ultra-diffuse galaxies (UDGs): evidence for two types of UDG? Mon. Not. R. Astron. Soc., 492(4):4874-4883, January 2020. 5.3.15

[64] M. Volonteri. Evolution of Supermassive Black Holes. In ESO Astrophysics Symposia, pages 174-182. Springer Berlin Heidelberg, 2007. 5.3.15

[65] Francesca Civano, Nico Cappelluti, Ryan Hickox, et al. Cosmic evolution of supermassive black holes: A view into the next two decades, May 2019. $\operatorname{Link}(2020)$ : https://ui.adsabs.harvard.edu/abs/2019BAAS...51c.429C/abstract. 5.3.15 
[66] Elizabeth Landau. Black hole seeds missing in cosmic garden. Jet Propulsion Laboratory News, September 2019. Link(2020): https://www.jpl.nasa.gov/news/news.php?feature=7504. 5.3.15

[67] Ana Bonaca, David W. Hogg, Adrian M. Price-Whelan, and Charlie Conroy. The Spur and the Gap in GD-1: Dynamical Evidence for a Dark Substructure in the Milky Way Halo. Astrophys. J., 880(1):38, July 2019. 5.3.16

[68] David Ehrenstein. Mapping Dark Matter in the Milky Way. Phys. Magazine, 12(51), May 2019. Link(2020): https://physics.aps.org/articles/v12/51. 5.3.16

[69] Lina Necib, Mariangela Lisanti, and Vasily Belokurov. Inferred Evidence for Dark Matter Kinematic Substructure with SDSS-Gaia. ApJ, 874(1):3, March 2019. 5.3.16

[70] Feryal Ozel, Dimitrios Psaltis, Ramesh Narayan, and Antonio Santos Villarreal. ON THE MASS DISTRIBUTION AND BIRTH MASSES OF NEUTRON STARS. Astrophys. J., 757(1):55, September 2012. 5.3.17

[71] Luciano Rezzolla, Elias R. Most, and Lukas R. Weih. Using Gravitational-wave Observations and Quasi-universal Relations to Constrain the Maximum Mass of Neutron Stars. Astrophys. J., 852(2):L25, January 2018. 5.3.17

[72] Heather Hill. Strange matter interacts strongly with nucleons. Phys. Today, 2020(1):0327a, March 2020. 5.3.17, 5.3.17

[73] Dana Najjar. 'Radical Change' Needed After Latest Neutron Star Collision. Quanta Magazine, February 2020. Link(2020): https://www.quantamagazine.org/radical-change-needed-after-latestneutron-star-collision-20200220/. 5.3.17, 5.3.17

[74] Mohammadtaher Safarzadeh, Enrico Ramirez-Ruiz, and Edo Berger. GW190425 is inconsistent with being a binary neutron star born from a fast merging channel. January 2020. Link(2020): https://inspirehep.net/literature/1775566. 5.3.17

[75] R. Abbott, T. D. Abbott, S. Abraham, et al. GW190814: Gravitational Waves from the Coalescence of a 23 Solar Mass Black Hole with a 2.6 Solar Mass Compact Object. Astrophys. J., 896(2):L44, June 2020. 5.3.17

[76] G. Risaliti and E. Lusso. Cosmological constraints from the Hubble diagram of quasars at high redshifts. Nature Astronomy, 3(3):272-277, January 2019. 5.3.18

[77] Daniel E. Holz, Scott A. Hughes, and Bernard F. Schutz. Measuring cosmic distances with standard sirens. Phys. Today, 71(12):34-40, December 2018. 6.3

[78] L. Verde, T. Treu, and A. G. Riess. Tensions between the early and late Universe. Nature Astronomy, 3(10):891-895, September 2019. 6.3

[79] Johanna L. Miller. Gravitational-lensing measurements push Hubble-constant discrepancy past $5 \sigma$. Phys. Today, 2020(1):0210a, February 2020. 6.3

[80] Thomas Lewton. What Might Be Speeding Up the Universe's Expansion? Quanta Magazizne, May 2020. Link(2020): https://www.quantamagazine.org/why-is-the-universe-expanding-so-fast$20200427 / .6 .3$

[81] Natalie Wolchover. New Wrinkle Added to Cosmology's Hubble Crisis. Quanta Magazine, February 2020. Link(2020): https://www.quantamagazine.org/new-wrinkle-added-to-cosmologys-hubblecrisis-20200226/. 6.3

[82] Wendy L. Freedman, Barry F. Madore, Taylor Hoyt, et al. Calibration of the Tip of the Red Giant Branch (TRGB). Astrophysical Journal, 891(1):57, March 2020. 6.3

[83] Vivian Poulin, Tristan L. Smith, Tanvi Karwal, and Marc Kamionkowski. Early Dark Energy can Resolve the Hubble Tension. Phys. Rev. Lett., 122(22):221301, June 2019. 6.3

[84] Sophie Ritson. The Many Dimensions of the String Theory Wars. phdthesis, The University of Sydney, May 2016. Link(2020): https://ses.library.usyd.edu.au/handle/2123/14968. 7.2 
[85] Meinard Kuhlmann. Quantum Field Theory. In Edward N. Zalta, editor, The Stanford Encyclopedia of Philosophy. Metaphysics Research Lab, Stanford University, winter 2018 edition, 2018. Link(2020): https://plato.stanford.edu/archives/win2018/entries/quantum-field-theory/. 8.1

[86] Abhay Deshpande and Rikutaro Yoshida. The Experiment That Will Probe the Deepest Recesses of the Atom. Sci. Amer., 320(6), June 2019. 8.1.3

[87] Charlie Wood. What Goes On in a Proton? Quark Math Still Conflicts With Experiments. Quanta, May 2020. Link(2020): https://www.quantamagazine.org/what-goes-on-in-a-proton-quark-mathstill-conflicts-with-experiments-20200506/. 8.1.3

[88] Maria Temming. This is the first known particle with four of the same kind of quark. Sci. News, 198(2), August 2020. Link(2020): https://www.sciencenews.org/article/new-particle-cerntetraquark-first-four-same-kind-quark. 8.1.3

[89] Marric Stephens. Synopsis: How a Pentaquark is Put Together. Physics Magazine, June 2019. Link(2020): https://physics.aps.org/synopsis-for/10.1103/PhysRevLett.122.222001. 8.1.3, 8.2.1

[90] Philip W. Anderson. Brainwashed by Feynman? Phys. Today, 53(2):11-12, February 2000. 8.1.4

[91] Z. K. Minev, S. O. Mundhada, S. Shankar, P. Reinhold, et al. To catch and reverse a quantum jump mid-flight. Nature, 570(7760):200-204, June 2019. 8.1.6

[92] R. Aaij, C. Abellan Beteta, B. Adeva, et al. Observation of a Narrow Pentaquark State, $P_{c}(4312)^{+}$, and of the Two-Peak Structure of the $P_{c}(4450)^{+}$. Phys. Rev. Lett., 122:222001, June 2019. 8.2.1

[93] G. A. Gonzalez-Sprinberg and J. Vidal. Tau magnetic moment. J. Phys. Conf. Ser., 912(1):012001, 2017. 8.2.2

[94] Teppei Kitahara, Takemichi Okui, Gilad Perez, et al. New Physics Implications of Recent Search for $\mathrm{KL} \rightarrow \pi 0 \nu^{-}$at KOTO. Phys. Rev. Lett., 124(7), February 2020. 9.1.2

[95] A. M. Sirunyan, A. Tumasyan, W. Adam, et al. Search for dark matter in events with a leptoquark and missing transverse momentum in proton-proton collisions at $13 \mathrm{TeV}$. Phys. Lett. B, 795:76-99, August 2019. 9.1.3

[96] Katherine Wright. Hints of Dark Bosons. Physics, September 2020. Link(2020): https://physics.aps.org/articles/v13/s115. 9.1.3

[97] Ian Counts, Joonseok Hur, Diana P. L. Aude Craik, et al. Evidence for Nonlinear Isotope Shift in $\mathrm{Yb}+$ Search for New Boson. Phys. Rev. Lett., 125(12), September 2020. 9.1.3

[98] Cyrille Solaro, Steffen Meyer, Karin Fisher, et al. Improved Isotope-Shift-Based Bounds on Bosons beyond the Standard Model through Measurements of the D23/2-D25/2 Interval in Ca+. Phys. Rev. Lett., 125(12), September 2020. 9.1.3

[99] Tanner Trickle, Zhengkang Zhang, and Kathryn M. Zurek. Detecting Light Dark Matter with Magnons. Physical Review Letters, 124(20), May 2020. 9.1.3

This essay: Copyright (c) 2020 Thomas J. Buckholtz 